\title{
Climate Phenomena and their Relevance for Future Regional Climate Change
}

\author{
Coordinating Lead Authors: \\ Jens Hesselbjerg Christensen (Denmark), Krishna Kumar Kanikicharla (India)
}

\section{Lead Authors:}

Edvin Aldrian (Indonesia), Soon-Il An (Republic of Korea), Iracema Fonseca Albuquerque Cavalcanti (Brazil), Manuel de Castro (Spain), Wenjie Dong (China), Prashant Goswami (India), Alex Hall (USA), Joseph Katongo Kanyanga (Zambia), Akio Kitoh (Japan), James Kossin (USA), Ngar-Cheung Lau (USA), James Renwick (New Zealand), David B. Stephenson (UK), Shang-Ping Xie (USA), Tianjun Zhou (China)

\section{Contributing Authors:}

Libu Abraham (Qatar), Tércio Ambrizzi (Brazil), Bruce Anderson (USA), Osamu Arakawa (Japan), Raymond Arritt (USA), Mark Baldwin (UK), Mathew Barlow (USA), David Barriopedro (Spain), Michela Biasutti (USA), Sébastien Biner (Canada), David Bromwich (USA), Josephine Brown (Australia), Wenju Cai (Australia), Leila V. Carvalho (USA/Brazil), Ping Chang (USA), Xiaolong Chen (China), Jung Choi (Republic of Korea), Ole Bøssing Christensen (Denmark), Clara Deser (USA), Kerry Emanuel (USA), Hirokazu Endo (Japan), David B. Enfield (USA), Amato Evan (USA), Alessandra Giannini (USA), Nathan Gillett (Canada), Annamalai Hariharasubramanian (USA), Ping Huang (China), Julie Jones (UK), Ashok Karumuri (India), Jack Katzfey (Australia), Erik Kjellström (Sweden), Jeff Knight (UK), Thomas Knutson (USA), Ashwini Kulkarni (India), Koteswara Rao Kundeti (India), William K. Lau (USA), Geert Lenderink (Netherlands), Chris Lennard (South Africa), Lai-yung Ruby Leung (USA), Renping Lin (China), Teresa Losada (Spain), Neil C. Mackellar (South Africa), Victor Magaña (Mexico), Gareth Marshall (UK), Linda Mearns (USA), Gerald Meehl (USA), Claudio Menéndez (Argentina), Hiroyuki Murakami (USA/Japan), Mary Jo Nath (USA), J. David Neelin (USA), Geert Jan van Oldenborgh (Netherlands), Martin Olesen (Denmark), Jan Polcher (France), Yun Qian (USA), Suchanda Ray (India), Katharine Davis Reich (USA), Belén Rodriguez de Fonseca (Spain), Paolo Ruti (Italy), James Screen (UK), Jan Sedláček (Switzerland) Silvina Solman (Argentina), Martin Stendel (Denmark), Samantha Stevenson (USA), Izuru Takayabu (Japan), John Turner (UK), Caroline Ummenhofer (USA), Kevin Walsh (Australia), Bin Wang (USA), Chunzai Wang (USA), lan Watterson (Australia), Matthew Widlansky (USA), Andrew Wittenberg (USA), Tim Woollings (UK), Sang-Wook Yeh (Republic of Korea), Chidong Zhang (USA), Lixia Zhang (China), Xiaotong Zheng (China), Liwei Zou (China)

\section{Review Editors:}

John Fyfe (Canada), Won-Tae Kwon (Republic of Korea), Kevin Trenberth (USA), David Wratt (New Zealand)

\section{This chapter should be cited as:}

Christensen, J.H., K. Krishna Kumar, E. Aldrian, S.-I. An, I.F.A. Cavalcanti, M. de Castro, W. Dong, P. Goswami, A. Hall, J.K. Kanyanga, A. Kitoh, J. Kossin, N.-C. Lau, J. Renwick, D.B. Stephenson, S.-P. Xie and T. Zhou, 2013: Climate Phenomena and their Relevance for Future Regional Climate Change. In: Climate Change 2013: The Physical Science Basis. Contribution of Working Group I to the Fifth Assessment Report of the Intergovernmental Panel on Climate Change [Stocker, T.F., D. Qin, G.-K. Plattner, M. Tignor, S.K. Allen, J. Boschung, A. Nauels, Y. Xia, V. Bex and P.M. Midgley (eds.)]. Cambridge University Press, Cambridge, United Kingdom and New York, NY, USA. 
Executive Summary

14.1 Introduction. 1222

14.1.1 Monsoons and Tropical Convergence Zones ...

14.1.2 Modes of Climate Variability 1222

14.1.3 Tropical and Extratropical Cyclones. 1223

14.1.4 Summary of Climate Phenomena and their Impact on Regional Climate. 1223

Box 14.1: Conceptual Definitions and Impacts of Modes of Climate Variability 1223

14.2 Monsoon Systems 1225

14.2.1

Global Overview 1225

14.2 .2

Asian-Australian Monsoon 1227

14.2 .3 American Monsoons 1232

14.2 .4

African Monsoon 1234

14.2 .5

Assessment Summary 1234

14.3 Tropical Phenomena. 1235

14.3.1

Convergence Zones 1235

14.3.2

Madden-Julian Oscillation..... 1237

14.3.3 Indian Ocean Modes 1237

14.3.4

Atlantic Ocean Modes. 1239

14.3 .5 Assessment Summary 1240

14.4 El Niño-Southern Oscillation 1240

14.4.1 Tropical Pacific Mean State 1240

14.4.2 El Niño Changes over Recent Decades and in the Future 1240

14.4.3

Teleconnections

14.4 .4

Assessment Summary

1243

14.5 Annular and Dipolar Modes. 1243

14.5.1 Northern Modes................................................. 1244

14.5.2 Southern Annular Mode ......................................... 1245

14.5.3 Assessment Summary ......................................... 1246

Box 14.2: Blocking. 1246

14.6 Large-scale Storm Systems 1248

14.6.1

14.6.2

Tropical Cyclones 1248

14.6 .3

Extratropical Cyclones 1251
14.7 Additional Phenomena of Relevance 1253

14.7.1 Pacific-South American Pattern 1253

14.7.2 Pacific-North American Pattern 1253

14.7.3 Pacific Decadal Oscillation/Inter-decadal Pacific Oscillation 1253

14.7.4 Tropospheric Biennial Oscillation ........................... 1253

14.7.5 Quasi-Biennial Oscillation...................................... 1254

14.7.6 Atlantic Multi-decadal Oscillation.......................... 1254

14.7.7 Assessment Summary .......................................... 1255

14.8 Future Regional Climate Change............................. 1255

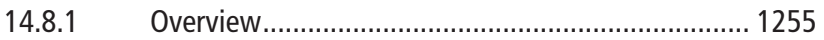

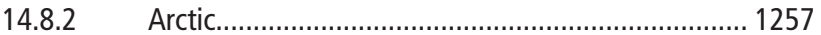

14.8.3 North America.................................................... 1258

14.8.4 Central America and Caribbean ............................. 1260

14.8.5 South America...................................................... 1261

14.8.6 Europe and Mediterranean .................................... 1264

14.8.7 Africa ...................................................................... 1266

14.8.8 Central and North Asia............................................ 1268

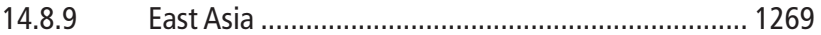

14.8.10 West Asia ........................................................... 1271

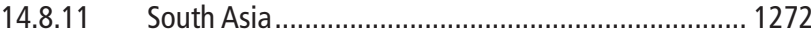

14.8.12 Southeast Asia ..................................................... 1273

14.8.13 Australia and New Zealand.................................. 1273

14.8.14 Pacific Islands Region............................................... 1275

14.8.15 Antarctica ........................................................... 1276

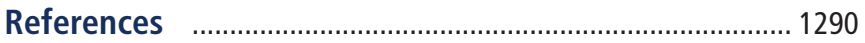

Frequently Asked Questions

FAQ 14.1 How Is Climate Change Affecting Monsoons?.

FAQ 14.2 How Are Future Projections in Regional Climate Related to Projections of Global Means? 1256

\section{Supplementary Material}

Supplementary Material is available in online versions of the report. 


\section{Executive Summary}

This chapter assesses the scientific literature on projected changes in major climate phenomena and more specifically their relevance for future change in regional climates, contingent on global mean temperatures continue to rise.

Regional climates are the complex result of processes that vary strongly with location and so respond differently to changes in global-scale influences. The following large-scale climate phenomena are increasingly well simulated by climate models and so provide a scientific basis for understanding and developing credibility in future regional climate change. A phenomenon is considered relevant to regional climate change if there is confidence that it has influence on the regional climate and there is confidence that the phenomenon will change, particularly under the Representative Concentration Pathway 4.5 (RCP4.5) or higher end scenarios. \{Table 14.3\}

\section{Monsoon Systems}

There is growing evidence of improved skill of climate models in reproducing climatological features of the global monsoon. Taken together with identified model agreement on future changes, the global monsoon, aggregated over all monsoon systems, is likely ${ }^{1}$ to strengthen in the 21st century with increases in its area and intensity, while the monsoon circulation weakens. Monsoon onset dates are likely to become earlier or not to change much and monsoon retreat dates are likely to delay, resulting in lengthening of the monsoon season in many regions. $\{14.2 .1\}$

Future increase in precipitation extremes related to the monsoon is very likely in South America, Africa, East Asia, South Asia, Southeast Asia and Australia. Lesser model agreement results in medium confidence $^{2}$ that monsoon-related interannual precipitation variability will increase in the future. $\{14.2 .1,14.8 .5,14.8 .7,14.8 .9,14.8 .11,14.8 .12$, 14.8.13\}

Model skill in representing regional monsoons is lower compared to the global monsoon and varies across different monsoon systems. There is medium confidence that overall precipitation associated with the Asian-Australian monsoon will increase but with a north-south asymmetry: Indian and East Asian monsoon precipitation is projected to increase, while projected changes in Australian summer monsoon precipitation are small. There is medium confidence that the Indian summer monsoon circulation will weaken, but this is compensated by increased atmospheric moisture content, leading to more precipitation. For the East Asian summer monsoon, both monsoon circulation and precipitation are projected to increase. There is medium confidence that the increase of the Indian summer monsoon rainfall and its extremes throughout the 21st century will be the largest among all monsoons. $\{14.2 .2,14.8 .9,14.8 .11,14.8 .13\}$

There is low confidence in projections of changes in precipitation amounts for the North American and South American monsoons, but medium confidence that the North American monsoon will arrive and persist later in the annual cycle, and high confidence in expansion of the South American monsoon area. $\{14.2 .3,14.8 .3,14.8 .4,14.8 .5\}$

There is low confidence in projections of a small delay in the development of the West African rainy season and an intensification of late-season rains. Model limitations in representing central features of the West African monsoon result in low confidence in future projections. $\{14.2 .4,14.8 .7\}$

\section{Tropical Phenomena}

Based on models' ability to reproduce general features of the Indian Ocean Dipole and agreement on future projections, the tropical Indian Ocean is likely to feature a zonal (east-west) pattern of change in the future with reduced warming and decreased precipitation in the east, and increased warming and increased precipitation in the west, directly influencing East Africa and Southeast Asia precipitation. $\{14.3,14.8 .7,14.8 .12\}$

A newly identified robust feature in model simulations of tropical precipitation over oceans gives medium confidence that annual precipitation change follows a 'warmer-get-wetter' pattern, increasing where warming of sea surface temperature exceeds the tropical mean and vice versa. There is medium confidence in projections showing an increase in seasonal mean precipitation on the equatorial flank of the Inter-Tropical Convergence Zone (ITCZ) affecting parts of Central America, the Caribbean, South Ameri$\mathrm{Ca}$, Africa and West Asia despite shortcomings in many models in simulating the ITCZ. There is medium confidence that the frequency of zonally oriented South Pacific Convergence Zone events will increase, with the South Pacific Convergence Zone (SPCZ) lying well to the northeast of its average position, a feature commonly reproduced in models that simulate the SPCZ realistically, resulting in reduced precipitation over many South Pacific island nations. Similarly there is medium confidence that the South Atlantic Convergence Zone will shift southwards, leading to an increase in precipitation over southeastern South America and a reduction immediately north thereof. $\{14.3,14.8 .4,14.8 .5$, $14.8 .7,14.8 .11,14.8 .14\}$

\footnotetext{
In this Report, the following terms have been used to indicate the assessed likelihood of an outcome or a result: Virtually certain 99-100\% probability, Very likely 90-100\%, Likely $66-100 \%$, About as likely as not 33-66\%, Unlikely 0-33\%, Very unlikely 0-10\%, Exceptionally unlikely 0-1\%. Additional terms (Extremely likely: 95-100\%, More likely than not $>50-100 \%$, and Extremely unlikely 0-5\%) may also be used when appropriate. Assessed likelihood is typeset in italics, e.g., very likely (see Section 1.4 and Box TS.1 for more details).

2 In this Report, the following summary terms are used to describe the available evidence: limited, medium, or robust; and for the degree of agreement: low, medium, or high. A level of confidence is expressed using five qualifiers: very low, low, medium, high, and very high, and typeset in italics, e.g., medium confidence. For a given evidence and agreement statement, different confidence levels can be assigned, but increasing levels of evidence and degrees of agreement are correlated with increasing confidence (see Section 1.4 and Box TS. 1 for more details).
} 
There is low confidence in projections of future changes in the Madden-Julian Oscillation owing to poor ability of the models to simulate it and its sensitivity to ocean warming patterns. The implications for future projections of regional climate extremes in West Asia, South Asia, Southeast Asia and Australia are therefore highly uncertain when associated with the Madden-Julian Oscillation. \{14.3, 14.8.10, 14.8.11, 14.8.12, 14.8.13\}

There is low confidence in the projections of future changes for the tropical Atlantic, both for the mean and interannual modes, because of systematic errors in model simulations of current climate. The implications for future changes in Atlantic hurricanes and tropical South American and West African precipitation are therefore uncertain. $\{14.3$, 14.6.1, 14.8.5, 14.8.7\}

The realism of the representation of El Niño-Southern Oscillation (ENSO) in climate models is increasing and models simulate ongoing ENSO variability in the future. Therefore there is high confidence that ENSO very likely remains as the dominant mode of interannual variability in the future and due to increased moisture availability, the associated precipitation variability on regional scales likely intensifies. An eastward shift in the patterns of temperature and precipitation variations in the North Pacific and North America related to El Niño and La Niña (teleconnections), a feature consistently simulated by models, is projected for the future, but with medium confidence, while other regional implications including those in Central and South America, the Caribbean, Africa, most of Asia, Australia and most Pacific Islands are more uncertain. However, natural modulations of the variance and spatial pattern of ENSO are so large in models that confidence in any specific projected change in its variability in the 21 st century remains low. $\{14.4,14.8 .3,14.8 .4$, $14.8 .5,14.8 .7,14.8 .9,14.8 .11,14.8 .12,14.8 .13,14.8 .14\}$

\section{Cyclones}

Based on process understanding and agreement in 21st century projections, it is likely that the global frequency of occurrence of tropical cyclones will either decrease or remain essentially unchanged, concurrent with a likely increase in both global mean tropical cyclone maximum wind speed and precipitation rates. The future influence of climate change on tropical cyclones is likely to vary by region, but the specific characteristics of the changes are not yet well quantified and there is low confidence in region-specific projections of frequency and intensity. However, better process understanding and model agreement in specific regions provide medium confidence that precipitation will be more extreme near the centres of tropical cyclones making landfall in North and Central America; East Africa; West, East, South and Southeast Asia as well as in Australia and many Pacific islands. Improvements in model resolution and downscaling techniques increase confidence in projections of intense storms, and the frequency of the most intense storms will more likely than not increase substantially in some basins. $\{14.6,14.8 .3,14.8 .4,14.8 .7,14.8 .9,14.8 .10,14.8 .11,14.8 .12,14.8 .13$, $14.8 .14\}$
Despite systematic biases in simulating storm tracks, most models and studies are in agreement on the future changes in the number of extratropical cyclones (ETCs). The global number of ETCs is unlikely to decrease by more than a few percent. A small poleward shift is likely in the Southern Hemisphere (SH) storm track. It is more likely than not, based on projections with medium confidence, that the North Pacific storm track will shift poleward. However, it is unlikely that the response of the North Atlantic storm track is a simple poleward shift. There is low confidence in the magnitude of regional storm track changes, and the impact of such changes on regional surface climate. It is very likely that increases in Arctic, Northern European, North American and SH winter precipitation by the end of the 21st century (2081-2100) will result from more precipitation in ETCs associated with enhanced extremes of storm-related precipitation. $\{14.6,14.8 .2,14.8 .3,14.8 .5,14.8 .6,14.8 .13,14.8 .15\}$

\section{Blocking}

Increased ability in simulating blocking in models and higher agreement on projections indicate that there is medium confidence that the frequency of Northern and Southern Hemisphere blocking will not increase, while trends in blocking intensity and persistence remain uncertain. The implications for blocking-related regional changes in North America, Europe and Mediterranean and Central and North Asia are therefore also uncertain. \{14.8.3, 14.8.6, 14.8.8, Box 14.2\}

\section{Annular and Dipolar Modes of Variability}

Models are generally able to simulate gross features of annular and dipolar modes. Model agreement in projections indicates that future boreal wintertime North Atlantic Oscillation is very likely to exhibit large natural variations and trend of similar magnitude to that observed in the past and is likely to become slightly more positive on average, with some, but not well documented, implications for winter conditions in the Arctic, North America and Eurasia. The austral summer/autumn positive trend in Southern Annular Mode is likely to weaken considerably as stratospheric ozone recovers through the mid-21st century with some, but not well documented, implications for South America, Africa, Australia, New Zealand and Antarctica. \{14.5.1, 14.5.2, 14.8.2, 14.8.3, 14.8.5, 14.8.6, 14.8.7, 14.8.8, 14.8.13, 14.8.15\}

\section{Atlantic Multi-decadal Oscillation}

Multiple lines of evidence from paleo reconstructions and model simulations indicate that the Atlantic Multi-decadal Oscillation (AMO) is unlikely to change its behaviour in the future as the mean climate changes. However, natural fluctuations in the AMO over the coming few decades are likely to influence regional climates at least as strongly as will human-induced changes, with implications for Atlantic major hurricane frequency, the West African wet season, North American and European summer conditions. \{14.7.6, 14.2.4, 14.6.1, 14.8.3, 14.8.6\} 


\section{Pacific South American Pattern}

Understanding of underlying physical mechanisms and the projected sea surface temperatures in the equatorial Indo-Pacific regions gives medium confidence that future changes in the mean atmospheric circulation for austral summer will project on this pattern, thereby influencing the South American Convergence Zone and precipitation over southeastern South America.

$\{14.7 .2,14.8 .5\}$ 


\subsection{Introduction}

Regional climates are the complex outcome of local physical processes and the non-local responses to large-scale phenomena such as the El Niño-Southern Oscillation (ENSO) and other dominant modes of climate variability. The dynamics of regional climates are determined by local weather systems that control the net transport of heat, moisture and momentum into a region. Regional climate is interpreted in the widest sense to mean the whole joint probability distribution of climate variables for a region including the time mean state, the variance and co-variance and the extremes.

This chapter assesses the physical basis of future regional climate change in the context of changes in the following types of phenomena: monsoons and tropical convergence zones, large-scale modes of climate variability and tropical and extratropical cyclones. Assessment of future changes in these phenomena is based on climate model projections (e.g., the Coupled Model Intercomparison Project Phase 3 (CMIP3) and CMIP5 multi-model ensembles described in Chapter 12) and an understanding of how well such models represent the key processes in these phenomena. More generic processes relevant to regional climate change, such as thermodynamic processes and landatmosphere feedback processes, are assessed in Chapter 12. Local processes such as snow-albedo feedback, moisture feedbacks due to local vegetation, effects of steep complex terrain etc. can be important for changes but are in general beyond the scope of this chapter. The main focus here is on large-scale atmospheric phenomena rather than more local feedback processes or impacts such as floods and droughts.

Sections 14.1.1 to 14.1.3 introduce the three main classes of phenomena addressed in this Assessment and then Section 14.1.4 summarizes their main impacts on precipitation and surface temperature. Specific climate phenomena are then addressed in Sections 14.2 to 14.7, which build on key findings from the Fourth Assessment Report, AR4 (IPCC, 2007a), and provide an assessment of process understanding and how well models simulate the phenomenon and an assessment of future projections for the phenomena. In Section 14.8, future regional climate changes are assessed, and where possible, interpreted in terms of future changes in phenomena. In particular, the relevance of the various phenomena addressed in this chapter for future climate change in the regions covered in Annex I are emphasized. The regions are those defined in previous regional climate change assessments (IPCC, 2007a, 2007b, 2012). Regional Climate Models (RCMs) and other downscaling tools required for local impact assessments are assessed in Section 9.6 and results from these studies are used where such supporting information adds additional relevant details to the assessment.

\subsubsection{Monsoons and Tropical Convergence Zones}

The major monsoon systems are associated with the seasonal movement of convergence zones over land, leading to profound seasonal changes in local hydrological cycles. Section 14.2 assesses current understanding of monsoonal behaviour in the present and future climate, how monsoon characteristics are influenced by the large-scale tropical modes of variability and their potential changes and how the monsoons in turn affect regional extremes. Convergence zones over the tropical oceans not only play a fundamental role in determining regional climates but also influence the global atmospheric circulation. Section 14.3 presents an assessment of these and other important tropical phenomena.

\subsubsection{Modes of Climate Variability}

Regional climates are strongly influenced by modes of climate variability (see Box 14.1 for definitions of mode, regime and teleconnection). This chapter assesses major modes such as El Niño-Southern Oscillation (ENSO, Section 14.4), the North Atlantic Oscillation/Northern Annular Mode (NAO/NAM) and Southern Annular Mode (SAM) in the extratropics (Section 14.5) and various other well-known modes such as the Pacific North American (PNA) pattern, Pacific Decadal Oscillation (PDO), Atlantic Multi-decadal Oscillation (AMO), etc. (Section 14.7). Many of these modes are described in previous IPCC reports (e.g., Section 3.6 of AR4 WG1). Chapter 2 gives operational definitions of mode indices (Box 2.5, Table 1) and an assessment of observed historical behaviour (Section 2.7.8). Climate models are generally able to simulate the gross features of many of the modes of variability (Section 9.5), and so provide useful tools for understanding how modes might change in the future (Müller and Roeckner, 2008; Handorf and Dethloff, 2009).

Modes and regimes provide a simplified description of variations in the climate system. In the simplest paradigm, variations in climate variables are described by linear projection onto a set of mode indices (Baldwin et al., 2009; Baldwin and Thompson, 2009; Hurrell and Deser, 2009). For example, a large fraction of interannual variance in Northern Hemisphere (NH) sea level pressure is accounted for by linear combinations of the NAM and the PNA modes (Quadrelli and Wallace, 2004). Alternatively, the nonlinear regime paradigm considers the probability distribution of local climate variables to be a multi-modal mixture of distributions related to a discrete set of regimes/types (Palmer, 1999; Cassou and Terray, 2001; Monahan et al., 2001).

There is ongoing debate on the relevance of the different paradigms (Stephenson et al., 2004; Christiansen, 2005; Ambaum, 2008; Fereday et al., 2008), and care is required when interpreting these constructs (Monahan et al., 2009; Takahashi et al., 2011).

Modes of climate variability may respond to climate change in one or more of the following ways:

- No change - the modes will continue to behave as they have done in the recent past.

- Index changes - the probability distributions of the mode indices may change (e.g., shifts in the mean and/or variance, or more complex changes in shape such as changes in local probability density, e.g., frequency of regimes).

- Spatial changes - the climate patterns associated with the modes may change spatially (e.g., new flavours of ENSO; see Section 14.4 and Supplementary Material) or the local amplitudes of the climate patterns may change (e.g., enhanced precipitation for a given change in index (Bulic and Kucharski, 2012)). 
- Structural changes - the types and number of modes and their mutual dependencies may change; completely new modes could in principle emerge.

An assessment of changes in modes of variability can be problematic for several reasons. First, interpretation depends on how one separates modes of variability from forced changes in the time mean or variations in the annual cycle (Pezzulli et al., 2005; Compo and Sardeshmukh, 2010). Modes of variability are generally defined using indices based on either detrended anomalies (Deser et al., 2010b) or anomalies obtained by removing the time mean over a historical reference period (see Box 2.5). The mode index in the latter approach will include changes in the mean, whereas by definition there is no trend in a mode index when it is based on detrended anomalies. Second, it can be difficult to separate natural variations from forced responses, for example, warming trends in the $\mathrm{N}$. Atlantic during the 20th century that may be due to trends in aerosol and other forcings rather than natural internal variability (see Sections 14.6.2 and 14.7.1). Finally, modes of climate variability are nonlinearly related to one another (Hsieh et al., 2006) and this relationship can change in time (e.g., trends in correlation between ENSO and NAO indices).

Even when the change in a mode of variability index does not contribute greatly to mean regional climate change, a climate mode may still play an important role in regional climate variability and extremes. Natural variations, such as those due to modes of variability, are a major source of uncertainty in future projections of mean regional climate (Deser et al., 2012). Furthermore, changes in the extremes of regional climate are likely to be sensitive to small changes in variance or shape of the distribution of the mode indices or the mode spatial patterns (Coppola et al., 2005; Scaife et al., 2008).

\subsubsection{Tropical and Extratropical Cyclones}

Tropical and extratropical cyclones (TCs and ETCs) are important weather phenomena intimately linked to regional climate phenomena and modes of climate variability. Both types of cyclone can produce extreme wind speeds and precipitation (see Section 3.4, IPCC Special Report on Managing the Risks of Extreme Events and Disasters to Advance Climate Change Adaptation (SREX; IPCC, 2012)). Sections 14.6.1 and 14.6.2 assess the recent progress in scientific understanding of how these important weather systems are likely to change in the future.

\subsubsection{Summary of Climate Phenomena and their Impact on Regional Climate}

Box 14.1, Figure 1 illustrates the large-scale climate phenomena assessed in this chapter. Many of the climate phenomena are evident in the map of annual mean rainfall (central panel). The most abundant annual rainfall occurs in the tropical convergence zones: Inter-Tropical Convergence Zone (ITCZ) over the Pacific, Atlantic and African equatorial belt (see Section 14.3.1.1), South Pacific Convergence Zone (SPCZ) over central South Pacific (see Section 14.3.1.2) and South Atlantic Convergence Zone (SACZ) over Southern South America and Southern Atlantic (see Section 14.3.1.3). In the global monsoon domain (white contours on the map), large amounts of precipitation occur but only in certain seasons (see Section 14.2.1). Local maxima in precipitation are also apparent over the major storm track regions in mid-latitudes (see Section 14.7.2). Box 14.1 Figure 1 also illustrates surface air temperature (left panels) and precipitation (right panels) teleconnection patterns for ENSO (in December to February and June to August; see Section 14.4), NAO (in December to February; see Section 14.5.1) and SAM (in September to November; see Section 14.5.2). The teleconnection patterns were obtained by taking the correlation between monthly gridded temperature and precipitation anomalies and indices for the modes (see Box 14.1 definitions). It can be seen that all three modes have far-reaching effects on temperature and precipitation in many parts of the world. Box 14.1, Table 1 briefly summarizes the main regional impacts of different well-known modes of climate variability.

\section{Box 14.1 | Conceptual Definitions and Impacts of Modes of Climate Variability}

This box briefly defines key concepts used to interpret modes of variability (below) and summarizes regional impacts associated with well-known modes (Box 14.1, Table 1 and Box 14.1, Figure 1). The terms below are used to describe variations in time series variables reported at a set of geographically fixed spatial locations, for example, a set of observing stations or model grid points (based on the more complete statistical and dynamical interpretation in Stephenson et al. (2004)).

\section{Climate indices}

Time series constructed from climate variables that provides an aggregate summary of the state of the climate system. For example, the difference between sea level pressure in Iceland and the Azores provides a simple yet useful historical NAO index (see Section 14.5 and Box 2.5 for definitions of this and other well-known observational indices). Because of their maximum variance properties, climate indices are often defined using principal components.

\section{Principal component}

A linear combination of a set of time series variables that has maximum variance subject to certain normalization constraints. Principal components are widely used to define optimal climate indices from gridded datasets (e.g., the Arctic Oscillation (AO) index, defined as the leading principal component of NH sea level pressure; Section 14.5). (continued on next page) 


\section{Box 14.1 (continued)}

\section{Climate pattern}

A set of coefficients obtained by 'projection' (regression) of climate variables at different spatial locations onto a climate index time series.

\section{Empirical Orthogonal Function}

The climate pattern obtained if the climate index is a principal component. It is an eigenvector of the covariance matrix of gridded climate data.

\section{Teleconnection}

A statistical association between climate variables at widely separated, geographically fixed spatial locations. Teleconnections are caused by large spatial structures such as basin-wide coupled modes of ocean-atmosphere variability, Rossby wave-trains, mid-latitude jets and storm-tracks, etc.

\section{Teleconnection pattern}

A correlation map obtained by calculating the correlation between variables at different spatial locations and a climate index. It is the special case of a climate pattern obtained for standardized variables and a standardized climate index, that is, the variables and index are each centred and scaled to have zero mean and unit variance. One-point teleconnection maps are made by choosing a variable at one of the locations to be the climate index. (continued on next page)

Box 14.1, Table 1| Regional climate impacts of fundamental modes of variability.

\begin{tabular}{|c|c|}
\hline Mode & Regional Climate Impacts \\
\hline ENSO & $\begin{array}{l}\text { Global impact on interannual variability in global mean temperature. Influences severe weather and tropical cyclone activity worldwide. The diverse El Niño } \\
\text { flavours present different teleconnection patterns that induce large impacts in numerous regions from polar to tropical latitudes (Section 14.4). }\end{array}$ \\
\hline PDO & $\begin{array}{l}\text { Influences surface air temperature and precipitation over the entire North American continent and extratropical North Pacific. Modulates ENSO rainfall } \\
\text { teleconnections, e.g., Australian climate (Section 14.7.3). }\end{array}$ \\
\hline IPO & $\begin{array}{l}\text { Modulates decadal variability in Australian rainfall, and ENSO teleconnections to rainfall, surface temperature, river flow and flood risk over Australia, } \\
\text { New Zealand and the SPCZ (Section 14.7.3). }\end{array}$ \\
\hline NAO & $\begin{array}{l}\text { Influences the N. Atlantic jet stream, storm tracks and blocking and thereby affects winter climate in over the N. Atlantic and surrounding landmasses. } \\
\text { The summer NAO (SNAO) influences Western Europe and Mediterranean basin climates in the season (Section 14.5.1). }\end{array}$ \\
\hline NAM & $\begin{array}{l}\text { Modulates the intensity of mid-latitude storms throughout the Northern Hemisphere and thereby influences North America and Eurasia climates as well as } \\
\text { sea ice distribution across the Arctic sea (Section 14.5.1). }\end{array}$ \\
\hline NPO & Influences winter air temperature and precipitation over much of western North America as well as Arctic sea ice in the Pacific sector (Section 14.5.1). \\
\hline SAM & $\begin{array}{l}\text { Influences temperature over Antarctica, Australia, Argentina, Tasmania and the south of New Zealand and precipitation over southern South America, } \\
\text { New Zealand, Tasmania, Australia and South Africa (Section 14.5.2). }\end{array}$ \\
\hline PNA & $\begin{array}{l}\text { Influences the jet stream and storm tracks over the Pacific and North American sectors, exerting notable influences on the temperature and precipitation in } \\
\text { these regions on intraseasonal and interannual time scales (Section 14.7.2). }\end{array}$ \\
\hline PSA & Influences atmospheric circulation over South America and thereby has impacts on precipitation over the continent (Section 14.7.1). \\
\hline AMO & $\begin{array}{l}\text { Influences air temperatures and rainfall over much of the Northern Hemisphere, in particular, North America and Europe. It is associated with multidecadal } \\
\text { variations in Indian, East Asian and West African monsoons, the North African Sahel and northeast Brazil rainfall, the frequency of North American droughts } \\
\text { and Atlantic hurricanes (Section 14.7.6). }\end{array}$ \\
\hline AMM & $\begin{array}{l}\text { Influences seasonal hurricane activity in the tropical Atlantic on both decadal and interannual time scales. Its variability is influenced by other modes, } \\
\text { particularly ENSO and NAO (Section 14.3.4). }\end{array}$ \\
\hline AN & $\begin{array}{l}\text { Affects the West African Monsoon, the oceanic forcing of Sahel rainfall on both decadal and interannual time-scales and the spatial extension of drought } \\
\text { in South Africa (Section 14.3.4). }\end{array}$ \\
\hline IOB & $\begin{array}{l}\text { Associated with the intensity of Northwest Pacific monsoon, the tropical cyclone activity over the Northwest Pacific and anomalous rainfall over East Asia } \\
\text { (Section 14.3.3). }\end{array}$ \\
\hline IOD & $\begin{array}{l}\text { Associated with droughts in Indonesia, reduced rainfall over Australia, intensified Indian summer monsoon, floods in East Africa, hot summers over Japan, and } \\
\text { anomalous climate in the extratropical Southern Hemisphere (Section 14.3.3). }\end{array}$ \\
\hline TBO & Modulates the strength of the Indian and West Pacific monsoons. Affects droughts and floods over large areas of south Asia and Australia (Section 14.7.4). \\
\hline MJO & $\begin{array}{l}\text { Modulates the intensity of monsoon systems around the globe and tropical cyclone activity in the Indian, Pacific and Atlantic Oceans. Associated with enhanced } \\
\text { rainfall in Western North America, northeast Brazil, Southeast Africa and Indonesia during boreal winter and Central America/Mexico and Southeast Asia } \\
\text { during boreal summer (Section 14.3.2). }\end{array}$ \\
\hline QBO & $\begin{array}{l}\text { Strongly affects the strength of the northern stratospheric polar vortex as well as the extratropical troposphere circulation, occurring preferentially } \\
\text { in boreal winter (Section 14.7.5). }\end{array}$ \\
\hline BLC & Associated with cold air outbreaks, heat-waves, floods and droughts in middle and high latitudes of both hemispheres (Box 14.2). \\
\hline
\end{tabular}

Notes:

AMM: Atlantic Meridional Mode

AMO: Atlantic Multi-decadal Oscillation

AN: Atlantic Niño pattern

BLC: Blocking events

ENSO: El Niño-Southern Oscillation
IOB: Indian Ocean Basin pattern IOD: Indian Ocean Dipole pattern IPO: Interdecadal Pacific Oscillation MJO: Madden-Julian Oscillation NAM: Northern Annular Mode
NAO: North Atlantic Oscillation NPO: North Pacific Oscillation PDO: Pacific Decadal Oscillation PNA: Pacific North America pattern PSA: Pacific South America pattern
QBO: Quasi-Biennial Oscillation SAM: Southern Annular Mode TBO: Tropospheric Biennial Oscillation 
Box 14.1 (continued)

\section{Mode of climate variability}

Underlying space-time structure with preferred spatial pattern and temporal variation that helps account for the gross features in variance and for teleconnections. A mode of variability is often considered to be the product of a spatial climate pattern and an associated climate index time series.

\section{Climate regime}

A set of similar states of the climate system that occur more frequently than nearby states due to either more persistence or more often recurrence. In other words, a cluster in climate state space associated with a local maximum in the probability density function.
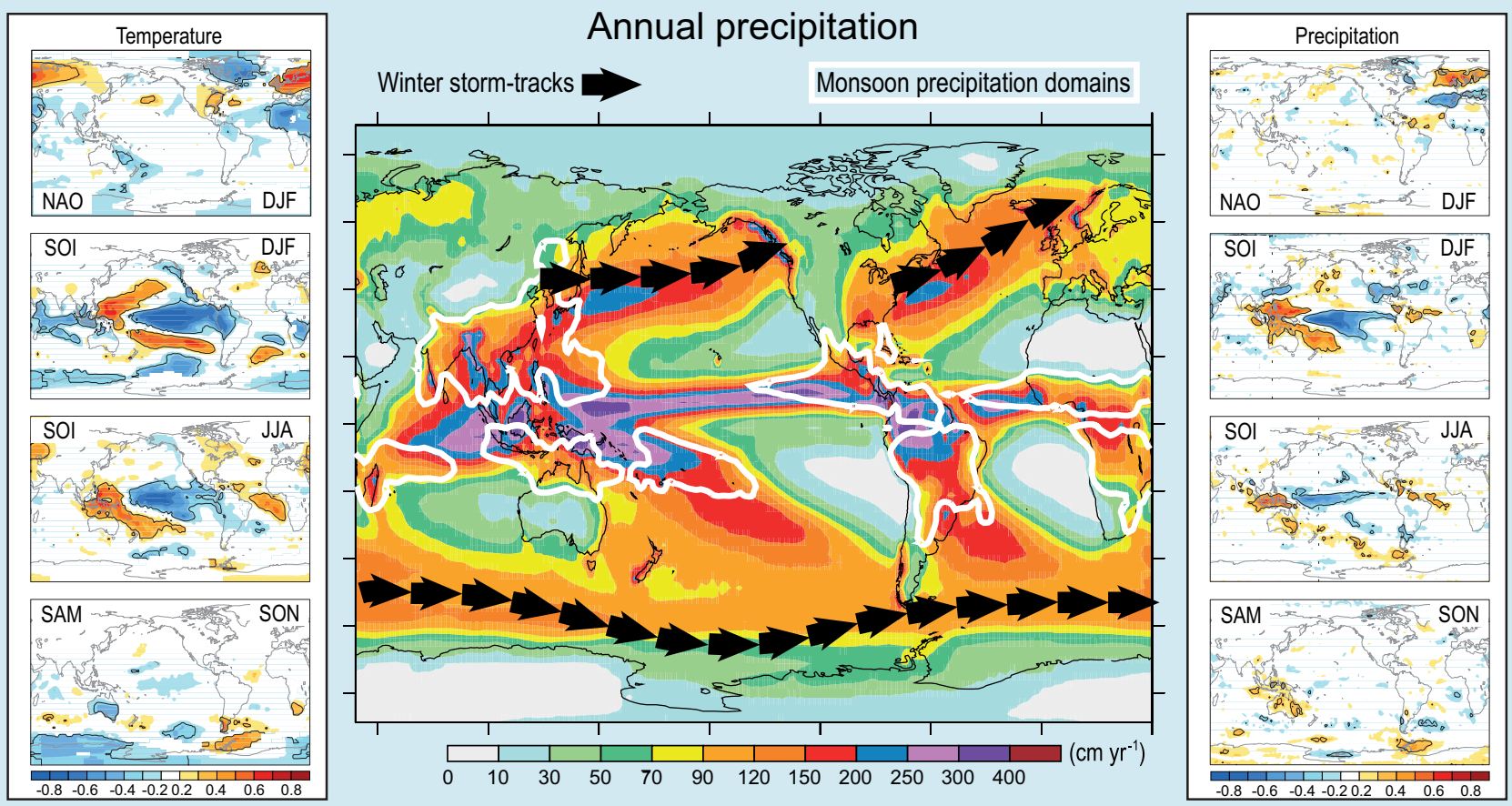

Box 14.1, Figure 1 | Global distribution of average annual rainfall (in cm/year) from 1979-2010 Global Precipitation Climatology Project (GPCP) database, monsoon precipitation domain (white contours) as defined in Section 14.2.1, and winter storm-tracks in both hemispheres (black arrows). In left (right) column seasonal correlation maps of North Atlantic Oscillation (NAO), Southern Oscillation Index (SOI, the atmospheric component of El Niño-Southern Oscillation (ENSO)) and Southern Annular Mode (SAM) mode indexes vs. monthly temperature (precipitation) anomalies in boreal winter (December, January and February (DJF)), austral winter (June, July and August (JJA)) and austral spring (September, October and November (SON)). Black contours indicate a 99\% significance level. The mode indices were taken from National Oceanic and Atmospheric Administration (NOAA, http://www.esrl.noaa.gov/psd/data/climateindices/list/), global temperatures from NASA Goddard Institute of Space Studies Surface Temperature Analysis (GISTEMP, http://data.giss.nasa.gov/gistemp/) and global precipitations from GPCP (http://www.esrl.noaa.gov/psd/data/ gridded/data.gpcp.html).

\subsection{Monsoon Systems}

Monsoons are a seasonal phenomenon responsible for producing the majority of wet season rainfall within the tropics. The precipitation characteristics over the Asian-Australian, American and African monsoons can be viewed as an integrated global monsoon system, associated with a global-scale atmospheric overturning circulation (Trenberth et al., 2000). In Section 14.2.1, changes in precipitation of the global monsoon system are assessed. Changes in regional monsoons are assessed in Sections 14.2.2 to 14.2.4.

\subsubsection{Global Overview}

The global land monsoon precipitation displays a decreasing trend over the last half-century, with primary contributions from the weakened summer monsoon systems in the NH (Wang and Ding, 2006). The combined global ocean-land monsoon precipitation has intensified during 1979-2008, mainly due to an upward trend in the $\mathrm{NH}$ summer oceanic monsoon precipitation (Zhou et al., 2008b; Hsu et al., 2011; Wang et al., 2012b). Because the fractional increase in monsoon area is greater than that in total precipitation, the ratio of the latter to the former (a measure of the global monsoon intensity) exhibits a 
decreasing trend (Hsu et al., 2011). CMIP5 models generally reproduce the observed global monsoon domain, but the disparity between the best and poorest models is large (Section 9.5.2.4).

In the CMIP5 models the global monsoon area (GMA), the global monsoon total precipitation (GMP) and the global monsoon precipitation intensity (GMI) are projected to increase by the end of the 21st century (2081-2100, Hsu et al., 2013; Kitoh et al., 2013; Figure 14.1). See Supplementary Material Section 14.SM.1.2 for the definitions of GMA, GMP and GMI. The CMIP5 model projections show an expansion of GMA mainly over the central to eastern tropical Pacific, the southern Indian Ocean and eastern Asia. In all RCP scenarios, GMA is very likely to increase, and GMI is likely to increase, resulting in a very likely increase in GMP, by the end of the 21st century (2081-2100). The 100 -year median changes in GMP are $+5 \%,+8 \%,+10 \%$, and $+16 \%$ in RCP2.6, RCP4.5, RCP6.0, and RCP8.5 scenarios, respectively. Indices of precipitation extremes such as simple daily precipitation intensity index (SDII), defined as the total precipitation divided by the number of days with precipitation greater than or equal to $1 \mathrm{~mm}$, annual max- imum 5-day precipitation total (R5d) and consecutive dry days (CDD) all indicate that intense precipitation will increase at larger rates than those of mean precipitation (Figure 14.1). The standard deviation of interannual variability in seasonal average precipitation (Psd) is projected to increase by many models but some models show a decrease in Psd. This is related to uncertainties in projections of future changes in tropical sea surface temperature (SST). Regarding seasonality, CMIP5 models project that monsoon onset dates will come earlier or not change much while monsoon retreat dates will delay, resulting in a lengthening of the monsoon season in many regions.

CMIP5 models show a decreasing trend of lower-troposphere wind convergence (dynamical factor) throughout the 20th and 21st centuries (Figure 14.2d). With increased moisture (see also Section 12.4), the moisture flux convergence shows an increasing trend from 1980 through the 21st century (Figure 14.2c). Surface evaporation shows a similar trend (Figure 14.2b) associated with warmer SSTs. Therefore, the global monsoon precipitation increases (Figure 14.2a) due to increases in moisture flux convergence and surface evaporation despite
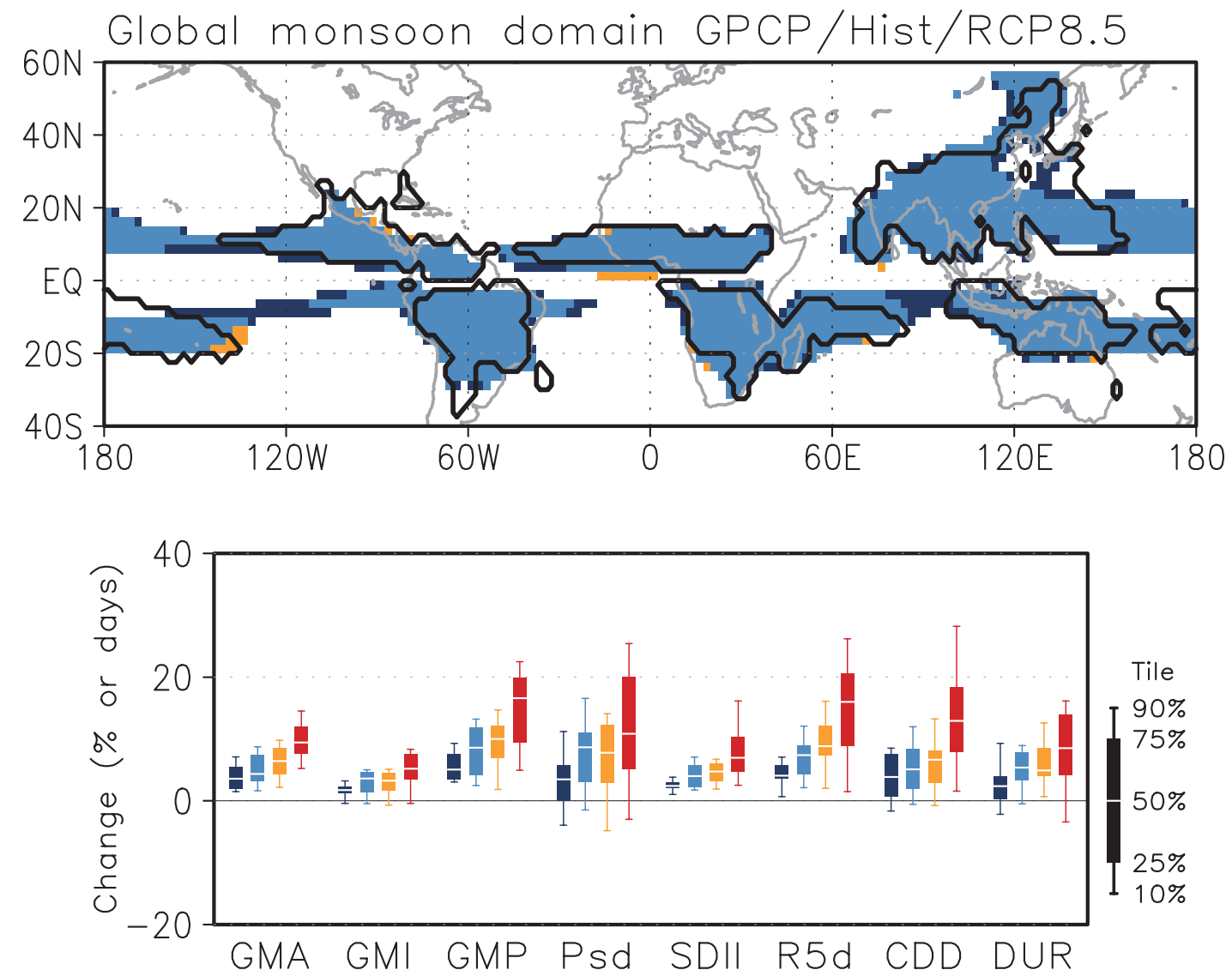

Figure 14.1 | (Upper) Observed (thick contour) and simulated (shading) global monsoon domain, based on the definition of Wang et al. (2011). The observations are based on GPCP v2.2 data (Huffman et al., 2009), and the simulations are based on 26 CMIP5 multi-model mean precipitation with a common 2.5 by 2.5 degree grid in the present day (1986-2005) and the future (2080-2099; RCP8.5 scenario). Orange (dark blue) shading shows monsoon domain only in the present day (future). Light blue shading shows monsoon domain in both periods. (Lower) Projected changes for the future (2080-2099) relative to the present day (1986-2005) in the global monsoon area (GMA) and global monsoon intensity (GMI), global monsoon total precipitation (GMP), standard deviation of interannual variability in seasonal average precipitation (Psd), simple daily precipitation intensity index (SDII), seasonal maximum 5-day precipitation total (R5d), seasonal maximum consecutive dry days (CDD) and monsoon season duration (DUR), under the RCP2.6 (dark blue; 18 models), RCP4.5 (light blue; 24 models), RCP6.0 (orange; 14 models) and RCP8.5 scenarios (red; 26 models). Units are \% except for DUR (days). Box-and-whisker plots show the 10th, 25th, 50th, 75th and 90th percentiles. All of the indices are calculated for the summer season (May to September in the Northern Hemisphere; November to March in the Southern Hemisphere). The indices of Psd, SDII, R5d and CDD calculated for each model's original grid, and then averaged over the monsoon domains determined by each model at the present-day. The indices of DUR are calculated for seven regional monsoon domains based on the criteria proposed by Wang and LinHo (2002) using regionally averaged climatological cycles of precipitation, and then their changes are averaged with weighting based on their area at the present day. 

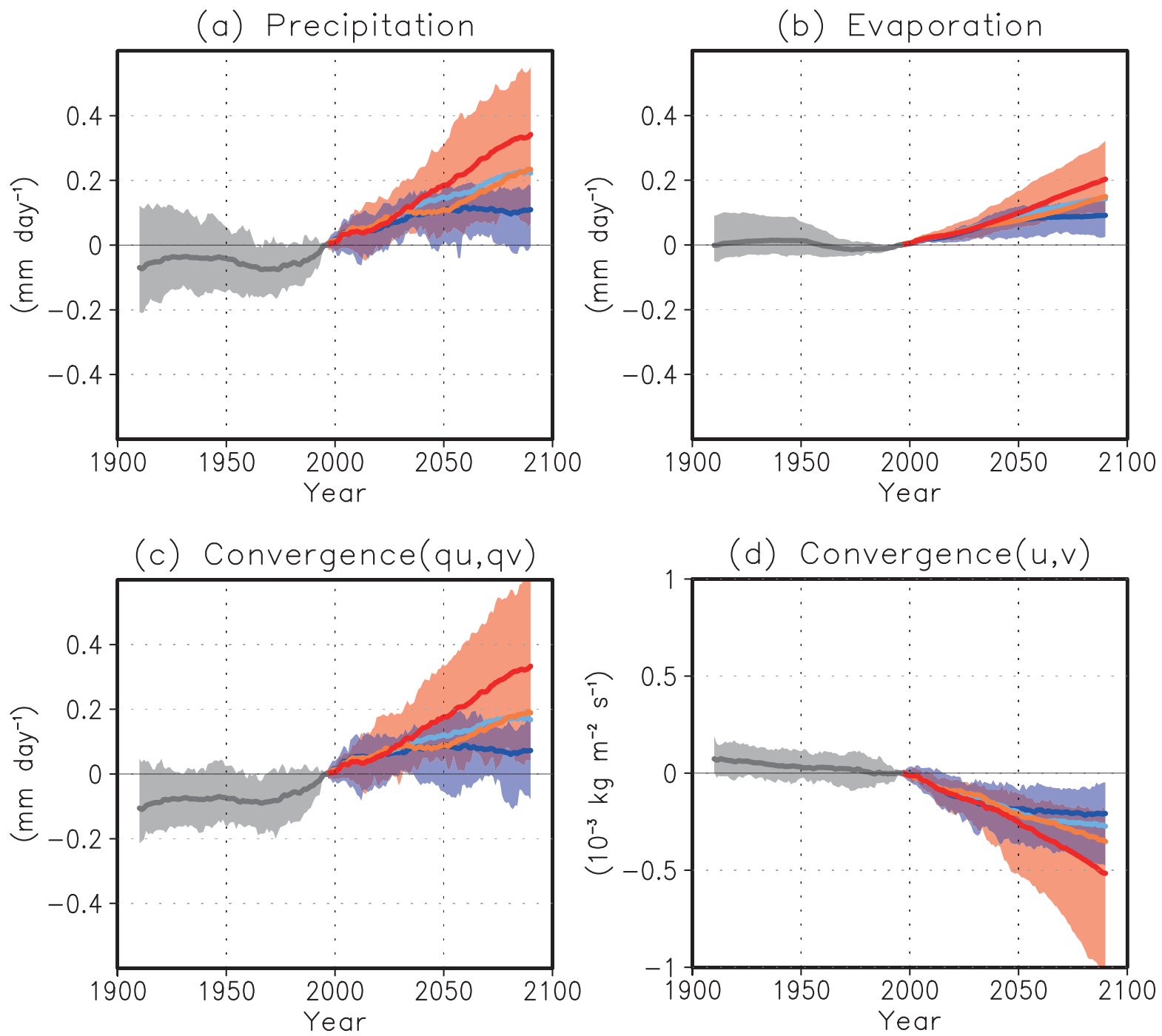

Figure 14.2 | Time series of simulated anomalies, smoothed with a 20-year running mean over the global land monsoon domain for (a) precipitation (mm day $\left.{ }^{-1}\right),(b)$ evaporation $\left(\mathrm{mm}\right.$ day $\left.{ }^{-1}\right)$, (c) water vapour flux convergence in the lower (below $500 \mathrm{hPa}$ ) troposphere $\left(\mathrm{mm}\right.$ day $\left.{ }^{-1}\right)$, and (d) wind convergence in the lower troposphere $\left(10^{-3} \mathrm{~kg} \mathrm{~m}^{-2} \mathrm{~s}^{-1}\right)$, relative to the present-day (1986-2005), based on CMIP5 multi-model monthly outputs. Historical (grey; 29 models), RCP2.6 (dark blue; 20 models), RCP4.5 (light blue; 24 models), RCP6.0 (orange; 16 models), and RCP8.5 (red; 24 models) simulations are shown in the 10th and 90th percentile (shading), and in all model averages (thick lines).

a weakened monsoon circulation. Besides greenhouse gases (GHGs), monsoons are affected by changes in aerosol loadings (Ramanathan et al., 2005). The aerosol direct forcing may heat the atmosphere but cools the surface, altering atmospheric stability and inducing horizontal pressure gradients that modulate the large-scale circulation and hence monsoon rainfall (Lau et al., 2008). However, the representation of aerosol forcing differs among models, and remains an important source of uncertainty (Chapter 7 and Section 12.2.2), particularly in some regional monsoon systems.

\subsubsection{Asian-Australian Monsoon}

The seasonal variation in the thermal contrast between the large Eurasian landmass and the Pacific-Indian Oceans drives the powerful Asian-Australian monsoon (AAM) system (Figure 14.3), which consists of five major subsystems: Indian (also known as South Asian), East Asian, Maritime Continent, Australian, and Western North Pacific monsoons. More than $85 \%$ of CMIP5 models show an increase in mean precipitation of the East Asian summer (EAS) monsoon, while more than $95 \%$ of models project an increase in heavy precipitation events (Figure 14.4). All models and all scenarios project an increase in both the mean and extreme precipitation in the Indian summer monsoon (referred to as SAS in Figures 14.3 and 14.4) . In these two regions, the interannual standard deviation of seasonal mean precipitation also increases. Over the Australian-Maritime Continent (AUSMC) monsoon region, agreement among models is low. Figure 14.5 shows the time-series of circulation indices representing EAS, Indian (IND), Western North Pacific (WNP) and Australian (AUS) summer monsoon systems. The Indian monsoon circulation index is likely to decrease in the 21st century, while a slight increase in the East Asian monsoon circulation is projected. Scatter among models is large for the western North Pacific and Australian monsoon circulation change.

Factors that limit the confidence in quantitative assessment of monsoon changes include sensitivity to model resolution (Cherchi and Navarra, 2007; Klingaman et al., 2011), model biases (Levine and 
Frequently Asked Questions

\section{FAQ 14.1 | How is Climate Change Affecting Monsoons?}

Monsoons are the most important mode of seasonal climate variation in the tropics, and are responsible for a large fraction of the annual rainfall in many regions. Their strength and timing is related to atmospheric moisture content, land-sea temperature contrast, land cover and use, atmospheric aerosol loadings and other factors. Overall, monsoonal rainfall is projected to become more intense in future, and to affect larger areas, because atmospheric moisture content increases with temperature. However, the localized effects of climate change on regional monsoon strength and variability are complex and more uncertain.

Monsoon rains fall over all tropical continents: Asia, Australia, the Americas and Africa. The monsoon circulation is driven by the difference in temperature between land and sea, which varies seasonally with the distribution of solar heating. The duration and amount of rainfall depends on the moisture content of the air, and on the configuration and strength of the atmospheric circulation. The regional distribution of land and ocean also plays a role, as does topography. For example, the Tibetan Plateau-through variations in its snow cover and surface heating-modulates the strength of the complex Asian monsoon systems. Where moist on-shore winds rise over mountains, as they do in southwest India, monsoon rainfall is intensified. On the lee side of such mountains, it lessens.

Since the late 1970s, the East Asian summer monsoon has been weakening and not extending as far north as it used to in earlier times, as a result of changes in the atmospheric circulation. That in turn has led to increasing drought in northern China, but floods in the Yangtze River Valley farther south. In contrast, the Indo-Australian and Western Pacific monsoon systems show no coherent trends since the mid-20th century, but are strongly modulated by the El Niño-Southern Oscillation (ENSO). Similarly, changes observed in the South American monsoon system over the last few decades are strongly related to ENSO variability. Evidence of trends in the North American monsoon system is limited, but a tendency towards heavier rainfalls on the northern side of the main monsoon region has been observed. No systematic long-term trends have been observed in the behaviour of the Indian or the African monsoons.

The land surface warms more rapidly than the ocean surface, so that surface temperature contrast is increasing in most regions. The tropical atmospheric overturning circulation, however, slows down on average as the climate warms due to energy balance constraints in the tropical atmosphere. These changes in the atmospheric circulation lead to regional changes in monsoon intensity, area and timing. There are a number of other effects as to how

(continued on next page)

(a) present

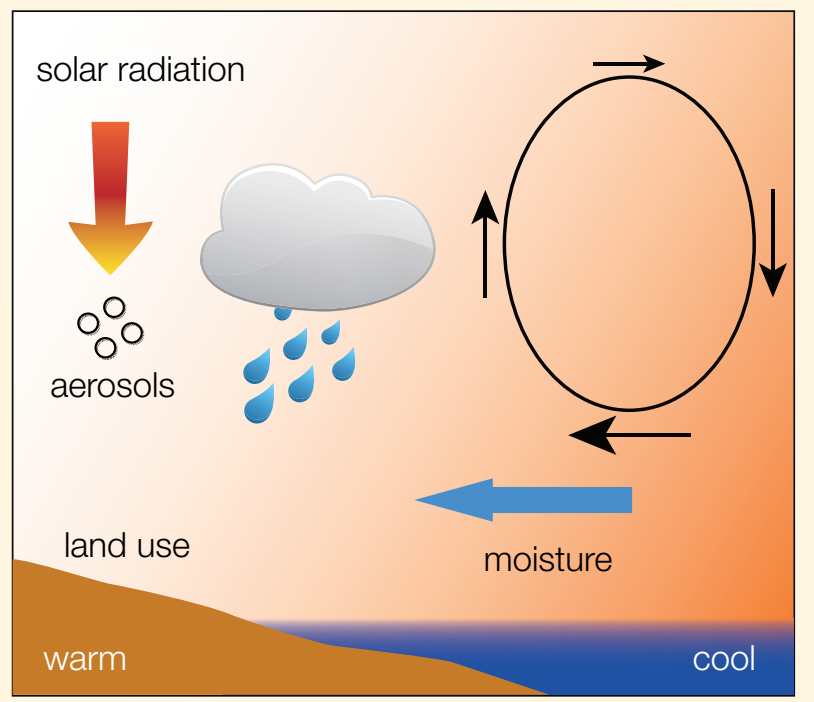

(b) future

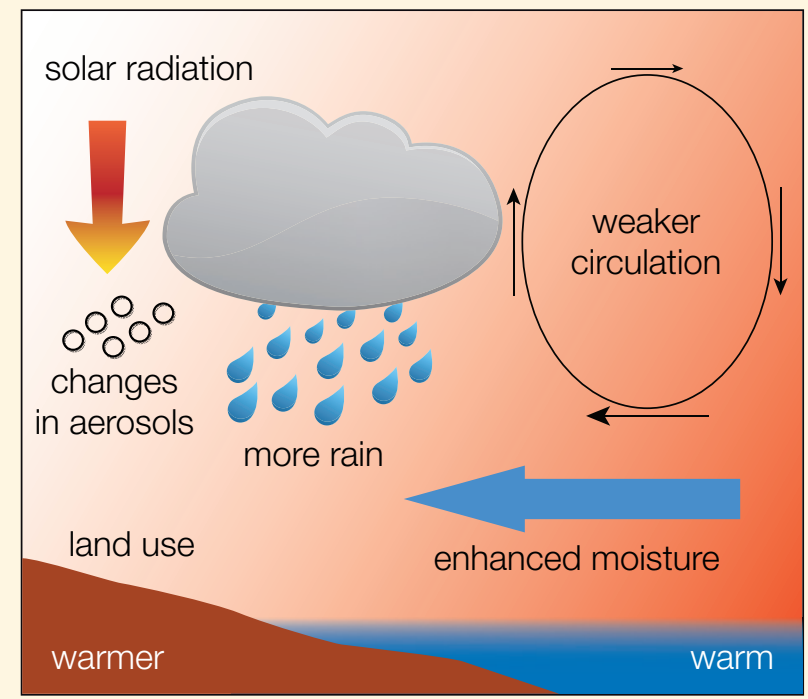

FAQ 14.1, Figure 1 | Schematic diagram illustrating the main ways that human activity influences monsoon rainfall. As the climate warms, increasing water vapour transport from the ocean into land increases because warmer air contains more water vapour. This also increases the potential for heavy rainfalls. Warming-related changes in large-scale circulation influence the strength and extent of the overall monsoon circulation. Land use change and atmospheric aerosol loading can also affect the amount of solar radiation that is absorbed in the atmosphere and land, potentially moderating the land-sea temperature difference. 


\section{FAQ 14.1 (continued)}

climate change can influence monsoons. Surface heating varies with the intensity of solar radiation absorption, which is itself affected by any land use changes that alter the reflectivity (albedo) of the land surface. Also, changing atmospheric aerosol loadings, such as air pollution, affect how much solar radiation reaches the ground, which can change the monsoon circulation by altering summer solar heating of the land surface. Absorption of solar radiation by aerosols, on the other hand, warms the atmosphere, changing the atmospheric heating distribution.

The strongest effect of climate change on the monsoons is the increase in atmospheric moisture associated with warming of the atmosphere, resulting in an increase in total monsoon rainfall even if the strength of the monsoon circulation weakens or does not change.

Climate model projections through the 21st century show an increase in total monsoon rainfall, largely due to increasing atmospheric moisture content. The total surface area affected by the monsoons is projected to increase, along with the general poleward expansion of the tropical regions. Climate models project from $5 \%$ to an approximately $15 \%$ increase of global monsoon rainfall depending on scenarios. Though total tropical monsoon rainfall increases, some areas will receive less monsoon rainfall, due to weakening tropical wind circulations. Monsoon onset dates are likely to be early or not to change much and the monsoon retreat dates are likely to delay, resulting in lengthening of the monsoon season.

Future regional trends in monsoon intensity and timing remain uncertain in many parts of the world. Year-to-year variations in the monsoons in many tropical regions are affected by ENSO. How ENSO will change in future-and how its effects on monsoon will change-also remain uncertain. However, the projected overall increase in monsoon rainfall indicates a corresponding increase in the risk of extreme rain events in most regions.

Turner, 2012; Bollasina and Ming, 2013), poor skill in simulating the Madden-Julian Oscillation (MJO; Section 9.1.3.3) and uncertainties in projected ENSO changes (Collins et al., 2010; Section 14.4) and in the representation of aerosol effects (Section 9.4.6).

\subsubsection{Indian Monsoon}

The Indian summer monsoon is known to have undergone abrupt shifts in the past millennium, giving rise to prolonged and intense droughts (Meehl and Hu, 2006; Sinha et al., 2011; see also Chapter 2). The observed recent weakening tendency in seasonal rainfall and the regional re-distribution has been partially attributed to factors such as changes in black carbon and/or sulphate aerosols (Chung and
Ramanathan, 2006; Lau et al., 2008; Bollasina et al., 2011), land use (Niyogi et al., 2010; see also Chapter 10) and SSTs (Annamalai et al., 2013). An increase in extreme rainfall events occurred at the expense of weaker rainfall events (Goswami et al., 2006) over the central Indian region, and in many other areas (Krishnamurthy et al., 2009). With a declining number of monsoon depressions (Krishnamurthy and Ajayamohan, 2010), the upward trend in extreme rainfall events may be due to enhanced moisture content (Goswami et al., 2006) or warmer SSTs in the tropical Indian Ocean (Rajeevan et al., 2008).

CMIP3 projections show suppressed rainfall over the equatorial Indian Ocean (Cai et al., 2011e; Turner and Annamalai, 2012), and an increase in seasonal mean rainfall over India (Ueda et al., 2006; Annamalai

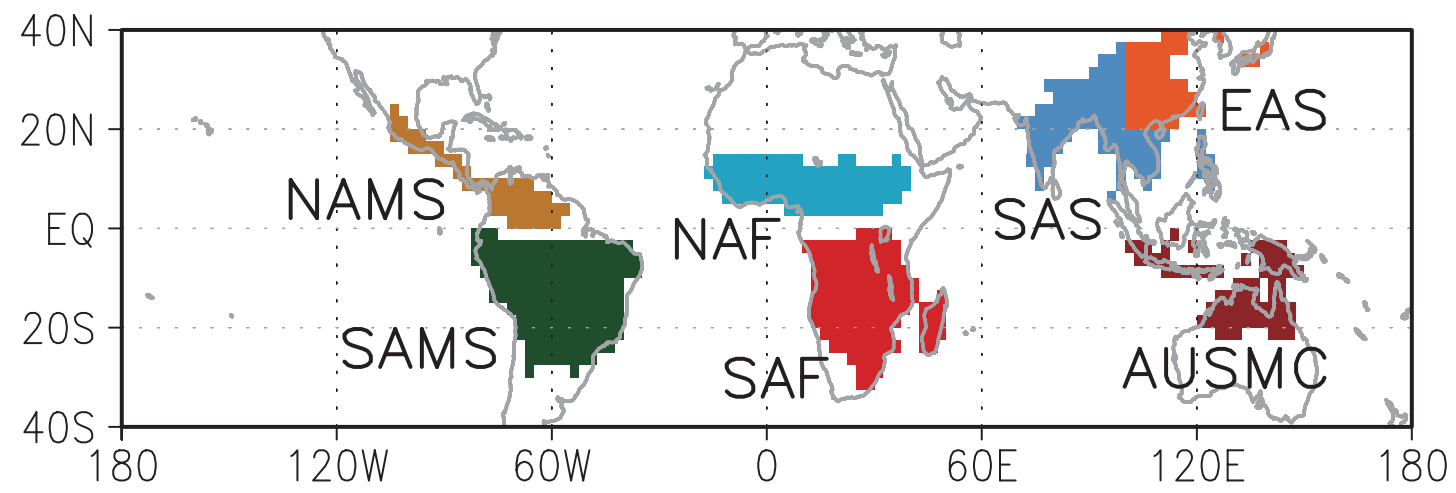

Figure 14.3 Regional land monsoon domain based on 26 CMIP5 multi-model mean precipitation with a common $2.5^{\circ} \times 2.5^{\circ}$ grid in the present-day (1986-2005). For regional divisions, the equator separates the northern monsoon domains (North America Monsoon System (NAMS), North Africa (NAF), Southern Asia (SAS) and East Asian summer (EAS)) from the southern monsoon domains (South America Monsoon System (SAMS), South Africa (SAF), and Australian-Maritime Continent (AUSMC)), $60^{\circ}$ E separates NAF from SAS, and $20^{\circ} \mathrm{N}$ and $100^{\circ} \mathrm{E}$ separates SAS from EAS. All the regional domains are within $40^{\circ} \mathrm{S}$ to $40^{\circ} \mathrm{N}$. 

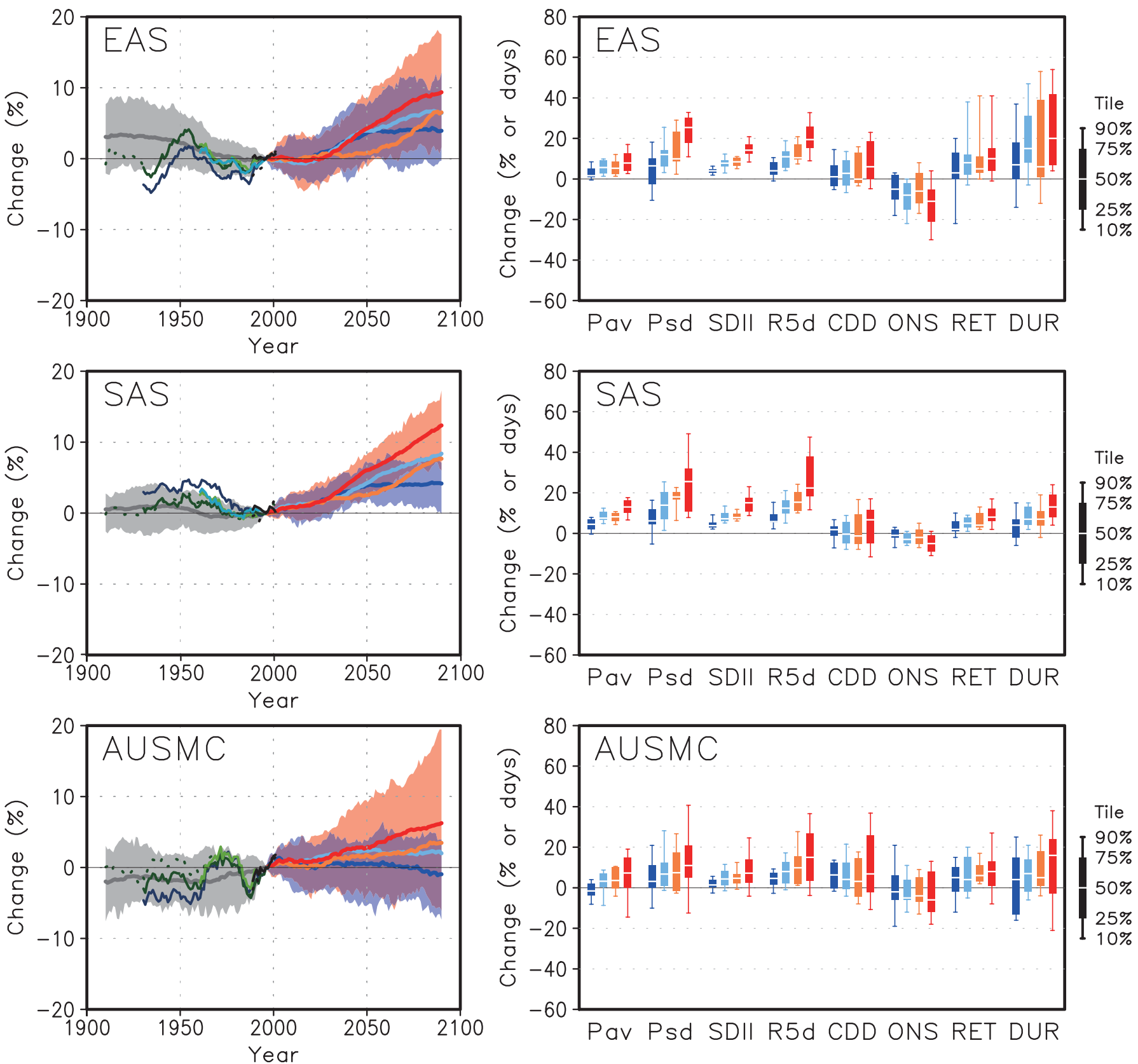

Figure 14.4 Changes in precipitation indices over the regional land monsoon domains of (upper) East Asian summer (EAS), (middle) Southern Asia (SAS), and (lower) AustralianMaritime Continent (AUSMC) based on CMIP5 multi-models. (Left) Time series of observed and model-simulated summer precipitation anomalies (\%) relative to the present-day average. All the time series are smoothed with a 20-year running mean. For the time series of simulations, all model averages are shown by thick lines for the historical (grey; 40 models), RCP2.6 (dark blue; 24 models), RCP4.5 (light blue; 34 models), RCP6.0 (orange; 20 models), and RCP8.5 scenarios (red; 32 models). Their intervals between 10 th and 90th percentiles are shown by shading for RCP2.6 and RCP8.5 scenarios. For the time series of observations, Climate Research Unit (CRU) TS3.2 (update from Mitchell and Jones, 2005; dark blue), Global Precipitation Climatology Centre (GPCC) v6 (Becker et al., 2013; deep green), GPCC Variability Analysis of Surface Climate Observations (VASClim0; Beck et al., 2005; light green), Highly Resolved Observational Data Integration Towards the Evaluation of Water Resources (APHRODITE) v1101 (Yatagai et al., 2012 ; only for EAS and SAS regions; light blue), Global Precipitation Climatology Project (GPCP) V2.2 (updated from Huffman et al., 2009; black), and Climate Prediction Center (NOAA) Merged Analysis of Precipitation (CMAP) v1201 (updated from Xie and Arkin, 1997; black with dots) are shown. GPCC v6 with dot line, GPCC VASClim0, GPCP v2.2 and CMAP v1201 are calculated using all grids for the period of 1901-2010, 1951-2000, 1979-2010, 1979-2010, respectively. CRU TS3.2, GPCC v6 with solid line, and APHRODITE v1101, are calculated using only grid boxes (2.5 in longitude/latitude) where at least one observation site exists for more than 80\% of the period of 1921-2005, 1921-2005, and 1951-2005, respectively. (Right) Projected changes for the future (2080-2099) relative to the present-day average in averaged precipitation (Pav), standard deviation of interannual variability in seasonal average precipitation (Psd), simple precipitation daily intensity index (SDII), seasonal maximum 5-day precipitation total (R5d), seasonal maximum consecutive dry days (CDD), monsoon onset date (ONS), retreat date (RET), and duration (DUR), under the RCP2.6 (18 models), RCP4.5 (24 models), RCP6.0 (14 models) and RCP8.5 scenarios (26 models). Units are \% in Pav, Psd, SDII, R5d, and CDD; days in ONS, RET, and DUR. Box-whisker plots show the 10th, 25th, 50th, 75th and 90th percentiles. All of the indices are calculated for the summer season (May to September in the Northern Hemisphere; November to March in the Southern Hemisphere). The indices of Pav, Psd, SDII, R5d and CDD are calculated for each model's original grid, and then averaged over the monsoon domains determined by each model at the present day. The indices of ONS, RET and DUR are calculated based on the criteria proposed by Wang and LinHo (2002) using regionally averaged climatological cycles of precipitation. 
(a)

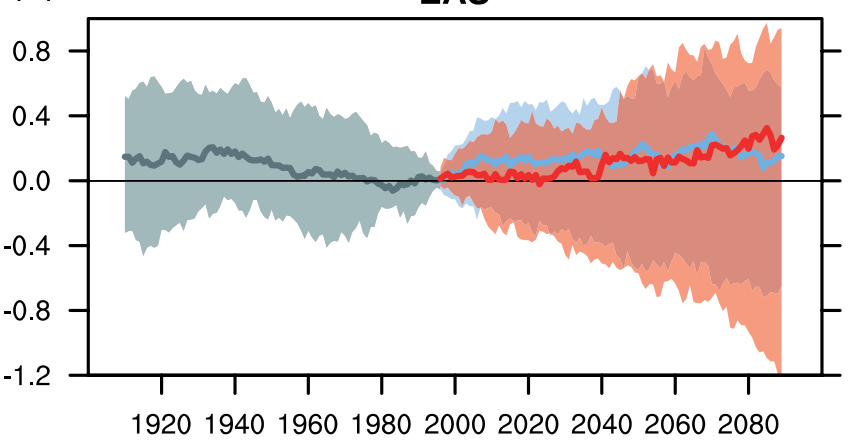

192019401960198020002020204020602080

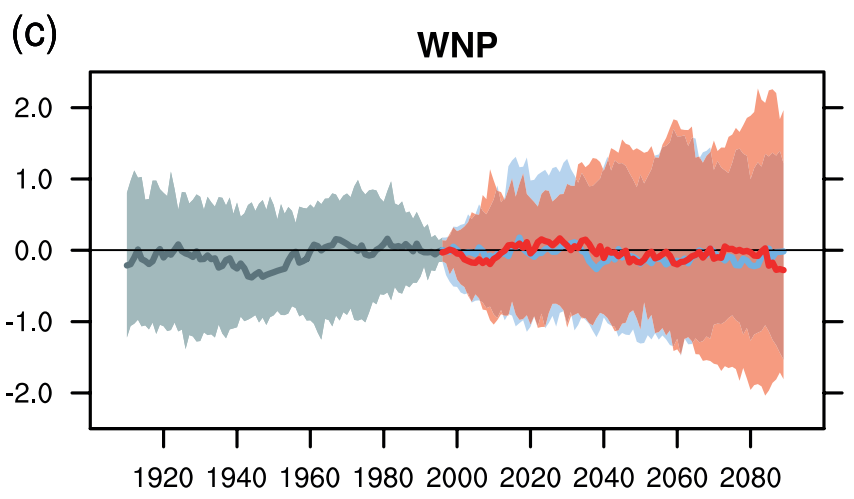

(b) SAS

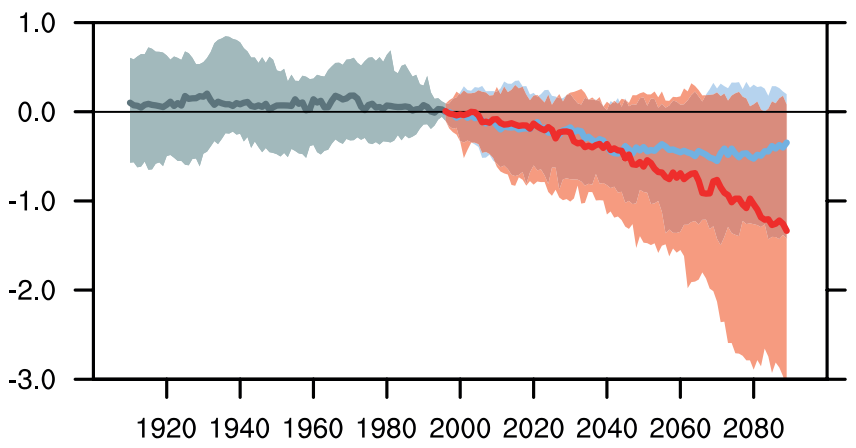

(d)

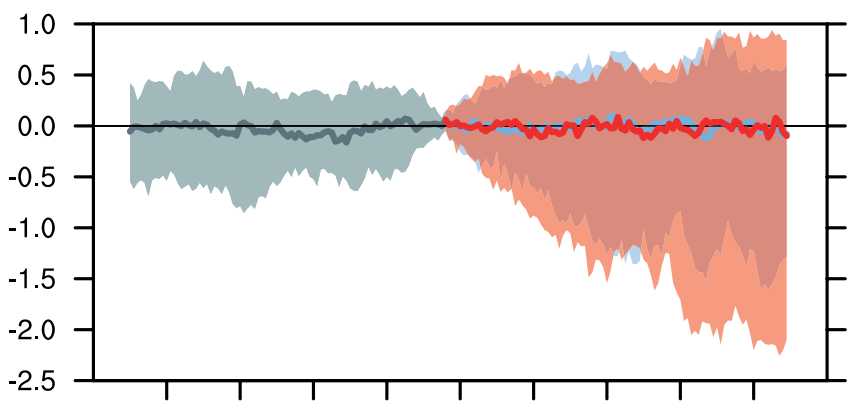

192019401960198020002020204020602080

Figure 14.5 | Time series of summer monsoon indices (21-year running mean) relative to the base period average (1986-2005). Historical (gray), RCP4.5 (light blue) and RCP8.5 (red) simulations by 39 CMIP5 model ensembles are shown in 10th and 90th (shading), and 50th (thick line) percentiles. (a) East Asian summer monsoon (defined as June, July and August (JJA) sea level pressure difference between $160^{\circ} \mathrm{E}$ and $110^{\circ} \mathrm{E}$ from $10^{\circ} \mathrm{N}$ to $50^{\circ} \mathrm{N}$ ), (b) Indian summer monsoon (defined as meridional differences of the JJA $850 \mathrm{hPa}$ zonal winds averaged over $5^{\circ} \mathrm{N}$ to $15^{\circ} \mathrm{N}, 40^{\circ} \mathrm{E}$ to $80^{\circ} \mathrm{E}$ and $20^{\circ} \mathrm{N}$ to $30^{\circ} \mathrm{N}, 60^{\circ} \mathrm{E}$ to $90^{\circ} \mathrm{E}$ ), (c) western North Pacific summer monsoon (defined as meridional differences of the JJA 850 $\mathrm{hPa}$ zonal winds averaged over $5^{\circ} \mathrm{N}$ to $15^{\circ} \mathrm{N}, 100^{\circ} \mathrm{E}$ to $130^{\circ} \mathrm{E}$ and $20^{\circ} \mathrm{N}$ to $30^{\circ} \mathrm{N}, 110^{\circ} \mathrm{E}$ to $140^{\circ} \mathrm{E}$ ), (d) Australian summer monsoon (defined as December, January and February (DJF) $850 \mathrm{hPa}$ zonal wind anomalies averaged over $10^{\circ} \mathrm{S}$ to $0^{\circ}, 120^{\circ} \mathrm{E}$ to $150^{\circ} \mathrm{E}$ ). (See Wang et al. (2004) and Zhou et al. (2009c) for indices definitions.)

et al., 2007; Turner et al., 2007a; Kumar et al., 2011b; Sabade et al., 2011). These results are generally confirmed by CMIP5 projections (Chaturvedi et al., 2012). The projected changes in Indian monsoon rainfall increase with the anthropogenic forcing among RCPs (May, 2011; see Figure 14.4; SAS).

In a suite of models that realistically simulate ENSO-monsoon relationships, normal monsoon years are likely to become less frequent in the future, but there is no clear consensus about the occurrence of extreme monsoon years (Turner and Annamalai, 2012). CMIP3 models indicate ENSO-monsoon relationships to persist in the future (Kumar et al., 2011b), but there is low confidence in the projection of ENSO variability (Section 14.4). Sub-seasonal scale monsoon variability is linked to the MJO but again the confidence in the future projection of MJO remains low (Section 14.3.2).

CMIP5 models project an increase in mean precipitation as well as its interannual variability and extremes (Figure 14.4; SAS). All models project an increase in heavy precipitation events but disagree on CDD changes. Regarding seasonality, model agreement is high on an earlier onset and later retreat, and hence longer duration. The monsoon circulation weakens in the future (Figure 14.5; IND) but the precipitation increases. Like the global monsoon (Section 14.2.1), the precipitation increase is largely due to the increased moisture flux from ocean to land.

\subsubsection{East Asian Monsoon}

The East Asian monsoon is characterized by a wet season and southerly flow in summer and by dry cold northerly flow in winter. The East Asian summer (EAS) monsoon circulation has experienced an inter-decadal weakening from the 1960s to the 1980s (Hori et al., 2007; Li et al., 2010a), associated with deficient rainfall in North China and excessive rainfall in central East China along $30^{\circ} \mathrm{N}(\mathrm{Hu}$, 1997; Wang, 2001; Gong and Ho, 2002; Yu et al., 2004). The summer monsoon circulation has begun to recover in recent decades (Liu et al., 2012a; Zhu et al., 2012). The summer rainfall amount over East Asia shows no clear trend during the 20th century (Zhang and Zhou, 2011), although significant trends may be found in local station records (Wang et al., 2006). The winter monsoon circulation weakened significantly after the 1980s (Wang et al., 2009a; Wang and Chen, 2010). See Supplementary Material Sections 14.SM.1.3 to 14.SM.1.7 for additional discussions of natural variability.

CMIP3 models show reasonable skill in simulating large-scale circulation of the EAS monsoon (Boo et al., 2011), but their performance is poor in reproducing the monsoon rainband (Lin et al., 2008a; Li and Zhou, 2011). Only a few CMIP3 models reproduce the Baiu rainband (Ninomiya, 2012) and high-resolution models (Kitoh and Kusunoki, 2008) show better performance than low resolution CMIP3 type models in simulating the monsoon rainband (Kitoh and Kusunoki, 
2008). CMIP3 models show large uncertainties in projections of monsoon precipitation and circulation (Ding et al., 2007; Kripalani et al., 2007a) but the simulation of interannual variability of the EAS monsoon circulation has improved from CMIP3 to CMIP5 (Sperber et al., 2012). Climate change may bring a change in the position of the monsoon rain band (Li et al., 2010a).

CMIP5 projections indicate a likely increase in both the circulation (Figure 14.5) and rainfall of the EAS monsoon (Figure 14.4) throughout the 21 st century. This is different from other Asian-Australian monsoon subsystems, where the increase in precipitation (Figure 14.4) is generally associated with weakening monsoon circulation (Figure 14.5). Interannual variability of seasonal mean rainfall is very likely to increase except for RCP2.6 (Figure 14.4). Heavy precipitation events (SDII and R5d) are also very likely to increase. CMIP5 models project an earlier monsoon onset and longer duration but the spread among models is large (Figure 14.4).

\subsubsection{Maritime Continent Monsoon}

Interaction between land and water characterizes the Maritime Continent region located between the Asian continent and Australia. It provides a land bridge along which maximum convection marches from the Asian summer monsoon regime (generally peaking in June, July and August) to the Australian summer monsoon system (generally peaking in December, January and February).

Phenomena such as the MJO (Tangang et al., 2008; Section 14.3.2; Hidayat and Kizu, 2010; Salahuddin and Curtis, 2011), and ENSO (Aldrian and Djamil, 2008; Moron et al., 2010; Section 14.4) influence Maritime Continent Monsoon variability. Rainfall extremes in the Maritime Continent are strongly influenced by diurnal rainfall variability (Qian, 2008; Qian et al., 2010a; Robertson et al., 2011; Ward et al., 2011) as well as the MJO. There have been no obvious trends in extreme rainfall indices in Indonesia, except evidence of a decrease in some areas in annual rainfall and an increase in the ratio of the wet to dry season rainfall (Aldrian and Djamil, 2008).

Modelling the Maritime Continent monsoon is a challenge because of the coarse resolution of contemporary large-scale coupled climate models (Aldrian and Djamil, 2008; Qian, 2008). Most CMIP3 models tend to simulate increasing precipitation in the tropical central Pacific but declining trends over the Maritime Continent for June to August (Ose and Arakawa, 2011), consistent with a decreasing zonal SST gradient across the equatorial Pacific and a weakening Walker Circulation (Collins et al., 2010). Projections of CMIP5 models are consistent with those of CMIP3 models, with decreasing precipitation during boreal summer and increasing precipitation during boreal winter, but model agreement is not high (Figures Al.66-67; Figure 12.22, but see also Figure 14.27).

\subsubsection{Australian Monsoon}

Some indices of the Australian summer monsoon (Wang et al., 2004; Li et al., 2012a) show a clear post-1980 reduction, but another index by Kajikawa et al. (2010) does not fully exhibit this change. Over northwest Australia, summer rainfall has increased by more than $50 \%$ (Rot- stayn et al., 2007; Shi et al., 2008b; Smith et al., 2008), whereas over northeast Australia, summer rainfall has decreased markedly since around 1980 (Li et al., 2012a).

Models in general show skill in representing the gross spatial characteristics of Australian monsoon summer precipitation (Moise et al., 2005). Further, atmospheric General Circulation Models (GCMs) forced by SST anomalies can skilfully reproduce monsoon-related zonal wind variability over recent decades (Zhou et al., 2009a). Recent analysis of the skill of a suite of CMIP3 models showed a good representation in the ensemble mean, but a very large range of biases across individual models (more than a factor of 6; Colman et al., 2011). Most CMIP models have biases in monsoon seasonality, but CMIP5 models generally perform better than CMIP3 (Jourdain et al., 2013).

In climate change projections, overall changes in tropical Australian rainfall are small, with substantial uncertainties (Figure 14.4; Moise et al., 2012; see also Figure 14.27). Using a group of CMIP5 models that exhibit a realistic present-day climatology, most projections using the RCP8.5 scenario produced $5 \%$ to $20 \%$ more monsoon rainfall by the late 21st century compared to the pre-industrial period (Jourdain et al., 2013). Most CMIP3 model projections suggest delayed monsoon onset and reduced monsoon duration over northern Australia. Weaker model agreement is seen over the interior of the Australian continent, where ensembles show an approximate 7-day delay of both the onset and retreat with little change in duration (Zhang et al., 2013a). CMIP5 model agreement in changes of monsoon precipitation seasonality is low (Figure 14.4).

\subsubsection{Western North Pacific Monsoon}

The western North Pacific summer monsoon (WNPSM) occupies a broad oceanic region of the South China and Philippine Seas, featuring a monsoon trough and a subtropical anticyclonic ridge to the north (Zhang and Wang, 2008).

The western North Pacific monsoon does not show any trend during 1950-1999. Since the late 1970s, the correlation has strengthened between interannual variability in the western North Pacific monsoon and ENSO (Section 14.4), a change mediated by Indian Ocean SST (Huang et al., 2010; Xie et al., 2010a). This occurred despite a weakening of the Indian monsoon-ENSO correlation in this period (Wang et al., 2008a).

CMIP5 models project little change in western North Pacific monsoon circulation (Figure 14.5) but enhanced precipitation (Figures Al.66-67; Figure 12.22; but see also Figure 14.24) due to increased moisture convergence (Chapter 12).

\subsubsection{American Monsoons}

The American monsoons, the North America Monsoon System (NAMS) and the South America Monsoon System (SAMS), are associated with large inter-seasonal differences in precipitation, humidity and atmospheric circulation (Vera et al., 2006; Marengo et al., 2010a). NAMS and SAMS indices are often, though not always, defined in terms of precipitation characteristics (Wang and LinHo, 2002). 

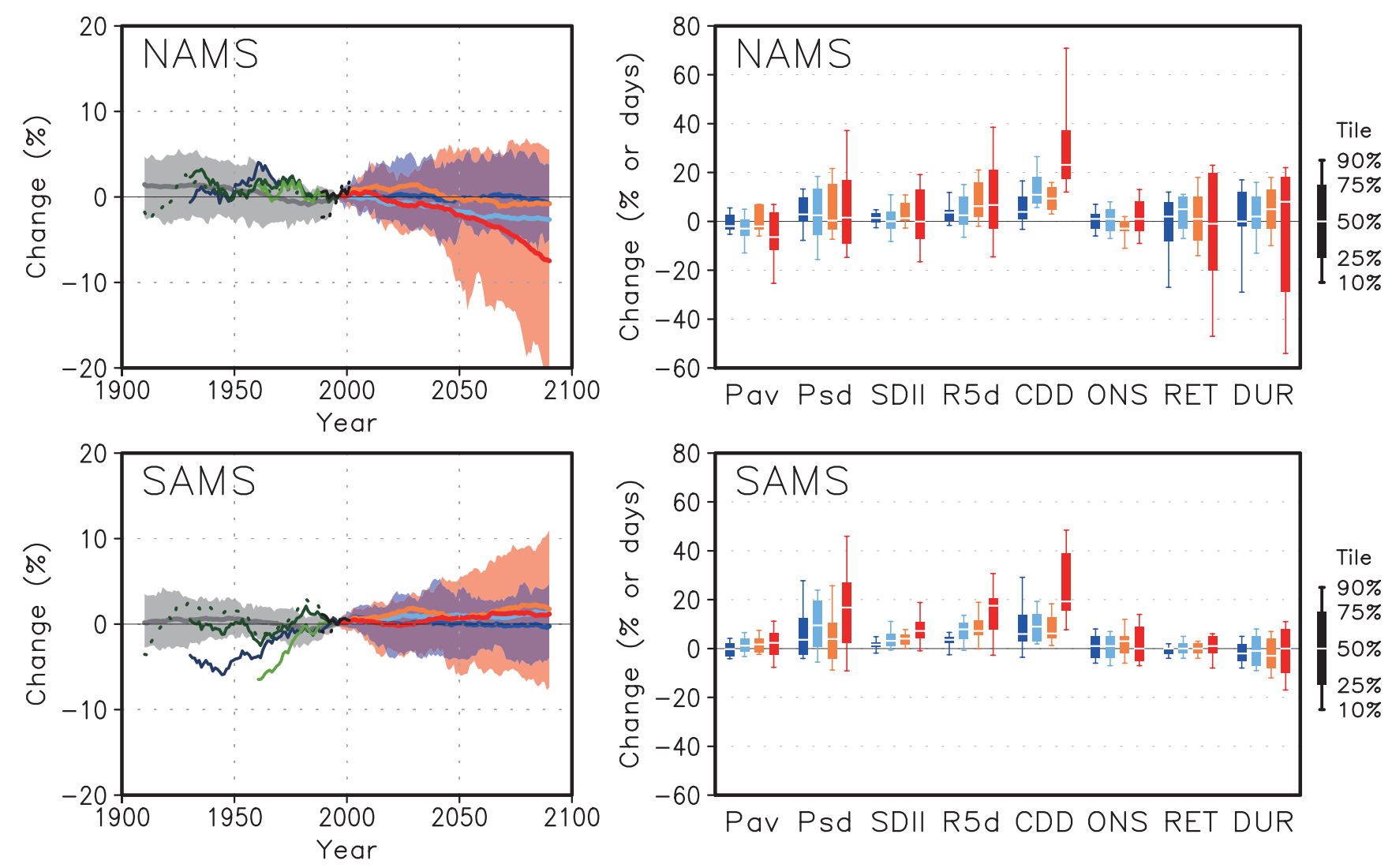

Figure 14.6 As in Figure 14.4, except for (upper) North America Monsoon System (NAMS) and (lower) South America Monsoon System (SAMS).

\subsubsection{North America Monsoon System}

The warm season precipitation in northern Mexico and the southwestern USA is strongly influenced by the NAMS. It has been difficult to simulate many important NAMS-related phenomenon in global climate models (Castro et al., 2007; Lin et al., 2008b; Cerezo-Mota et al., 2011), though the models capture gross-scale features associated with the NAMS seasonal cycle (Liang et al., 2008b; Gutzler, 2009). See Supplementary Material Section 14.SM.1.8 for a more detailed discussion of NAMS dynamics.

In the NAMS core region, no distinct precipitation trends have been seen over the last half of the 20th century (Anderson et al., 2010; Arriaga-Ramirez and Cavazos, 2010), due to countervailing trends in increasing intensity and decreasing frequency of events, as well as the decreasing length of the monsoon season itself (Englehart and Douglas, 2006). However, monsoonal stream flow in western Mexico has been decreasing, possibly as a result of changing precipitation characteristics or antecedent hydrological conditions rather than overall precipitation amounts (Gochis et al., 2007). There has also been a systematic delay in monsoon onset, peak and termination (Grantz et al., 2007) as well as an increase in extreme precipitation events associated with land-falling hurricanes (Cavazos et al., 2008). Finally, positive trends in NAMS precipitation amounts have been detected in the northern fringes of the core area, that is, Arizona and western New Mexico (Anderson et al., 2010), consistent with northward NAMS expansion during relatively warm periods in the Holocene (Petersen,
1994; Mock and Brunelle-Daines, 1999; Harrison et al., 2003; Poore et al., 2005; Metcalfe et al., 2010).

Over the coming century, CMIP5 simulations generally project a precipitation reduction in the core zone of the monsoon (Figures Al.27 and Figure 14.6), but this signal is not particularly consistent across models, even under the RCP8.5 scenario (Cook and Seager, 2013). Thus confidence in projections of monsoon precipitation changes is currently low. CMIP5 models have no consensus on future changes of monsoon timing (Figure 14.6). Temperature increases are consistently projected in all models (Annex I). This will likely increase the frequency of extreme summer temperatures (Diffenbaugh and Ashfaq, 2010; Anderson, 2011; Duffy and Tebaldi, 2012), together with projected increase in consecutive dry days (Figure 14.6).

\subsubsection{South America Monsoon System}

The SAMS mainly influences precipitation in the South American tropics and subtropics (Figure 14.1). The main characteristics of SAMS onset are increased humidity flux from the Atlantic Ocean over northern South America, an eastward shift of the subtropical high, strong northwesterly moisture flux east of the tropical Andes, and establishment of the Bolivian High (Raia and Cavalcanti, 2008; Marengo et al., 2010a; Silva and Kousky, 2012). Recent SAMS indices have been calculated based on different variables, such as a large scale index (Silva and Carvalho, 2007), moisture flux (Raia and Cavalcanti, 2008), and wind (Gan et al., 2006), in addition to precipitation (Nieto-Ferreira and 
Rickenbach, 2010; Seth et al., 2010; Kitoh et al., 2013). As seen below, conclusions regarding SAMS changes can depend on the index chosen.

SAMS duration and amplitude obtained from the observed large-scale index have both increased in the last 32 years (Jones and Carvalho, 2013). Increase of extreme precipitation and consecutive dry days have been observed in the SAMS region from 1969 to 2009 (Skansi et al., 2013). The overall annual cycle of precipitation in the SAMS region, including SAMS onset and demise, is generally well represented by models (Bombardi and Carvalho, 2009; Seth et al., 2010; Kitoh et al., 2013). Extreme precipitation indices in SAMS region are also well simulated by CMIP5 models (Kitoh et al., 2013). CMIP5 models subjected to historical forcing show increases in SAMS amplitude, earlier onset and later demise during the 1951-2005 period (Jones and Carvalho, 2013). Using a precipitation based index, precipitation increases in austral summer but decreases in austral spring, indicating delayed SAMS onset in the CMIP3 projections (Seth et al., 2011). CMIP5 projections based on the global precipitation index (Section 14.2.1) consistently show small precipitation increases and little change in onset and retreat (Kitoh et al., 2013; Figure 14.6). On the other hand, when using a different index, earlier onsets and later demises and thus, longer duration of the SAMS by the end of the 21st century (2081-2100) has been found (Jones and Carvalho, 2013). Thus there is medium confidence that SAMS overall precipitation will remain unchanged. The different estimates of changes in timing underscores potential uncertainties related to SAMS timing due to differences in SAMS indices. The models do show significant and robust increases in extreme precipitation indices in the SAMS region, such as seasonal maximum 5-day precipitation total and number of consecutive dry days (Figure 14.6), leading to medium confidence in projections of these characteristics.

\subsubsection{African Monsoon}

In Africa, monsoon circulation affects precipitation in West Africa where notable upper air flow reversals are observed. East and south African precipitation is generally described by variations in the tropical convergence zone rather than as a monsoon feature. This section covers the West African monsoon, and Section 14.8 .7 also covers the latter two regions.

The West African monsoon develops during northern spring and summer, with a rapid northward jump of the rainfall belt from along the Gulf of Guinea at $5^{\circ} \mathrm{N}$ in May to June to the Sahel at $10^{\circ} \mathrm{N}$ in July to August. Factors influencing the West African monsoon include interannual to decadal variations, land processes and the direct response to radiative forcing. Cross-equatorial tropical Atlantic SST patterns influence the monsoon flow and moistening of the boundary layer, so that a colder northern tropical Atlantic induces negative rainfall anomalies (Biasutti et al., 2008; Giannini et al., 2008; Xue et al., 2010; Rowell, 2011).

In CMIP3 simulations, rainfall is projected to decrease in the early part but increase towards the end of the rainy season, implying a small delay in the monsoon season and an intensification of late-season rains (Biasutti and Sobel, 2009; Biasutti et al., 2009; Seth et al., 2010). CMIP5 models, on the other hand, simulate the variability of tropical Atlantic SST patterns with little credibility (Section 9.4.2.5.2) and model resolu- tion is known to limit the ability to capture the mesoscale 'squall line' systems that form a central element in the maintenance of the rainy season (Ruti and Dell'Aquila, 2010; see also Section 14.8.7). Therefore, projections of the West African monsoon rainfall appear to be uncertain, reflected by considerable model deficiencies and spread in the projections (Figure 14.7). Note that this figure is based on a somewhat eastward extended area for the West African monsoon (NAF in Figure 14.3), seen as a component of the global monsoon system (Section 14.2.1). The limitations of model simulations in the region arising from the lack of convective organization (Kohler et al., 2010) leading to the underestimation of interannual variability (Scaife et al., 2009) imply that confidence in projections of the African monsoon is low.

The limited information that could be deduced from CMIP3 has not improved much in CMIP5. Figure 14.7 largely confirms the findings based on CMIP3. The CMIP5 model ensemble projects a modest change in the onset date (depending on the scenario) and a small delay in the retreat date, leading to a small increase in the duration of the rainy season. The delay in the monsoon retreat is larger in the high-end emission scenarios. The interannual variance and the 5-day rain intensity show a robust increase, while a small increase in dry day periods is less significant.

\subsubsection{Assessment Summary}

It is projected that global monsoon precipitation will likely strengthen in the 21st century with increase in its area and intensity while the monsoon circulation weakens. Precipitation extremes including precipitation intensity and consecutive dry days are likely to increase at higher rates than those of mean precipitation. Overall, CMIP5 models project that the monsoon onset will be earlier or not change much and the monsoon retreat dates will delay, resulting in a lengthening of the monsoon season. Such features are likely to occur in most of Asian-Australian Monsoon regions.

There is medium confidence that overall precipitation associated with the Asian-Australian monsoon will increase but with a north-south asymmetry: Indian and East Asian monsoon precipitation is projected to increase, while projected changes in Australian summer monsoon precipitation are small. There is medium confidence that the Indian summer monsoon circulation will weaken, but this is compensated by increased atmospheric moisture content, leading to more precipitation. For the East Asian summer monsoon, both monsoon circulation and precipitation are projected to increase. There is low confidence that over the Maritime Continent boreal summer rainfall will decrease and boreal winter rainfall will increase. There is low confidence that changes in the tropical Australian monsoon rainfall are small. There is low confidence that Western North Pacific summer monsoon circulation changes are small, but with increased rainfall due to enhanced moisture. There is medium confidence in an increase of Indian summer monsoon rainfall and its extremes throughout the 21st century under all RCP scenarios. Their percentage change ratios are the largest and model agreement is highest among all monsoon regions.

There is low confidence in projections of American monsoon precipitation changes but there is high confidence in increases of precipitation extremes, of wet days and consecutive dry days. There is medium 

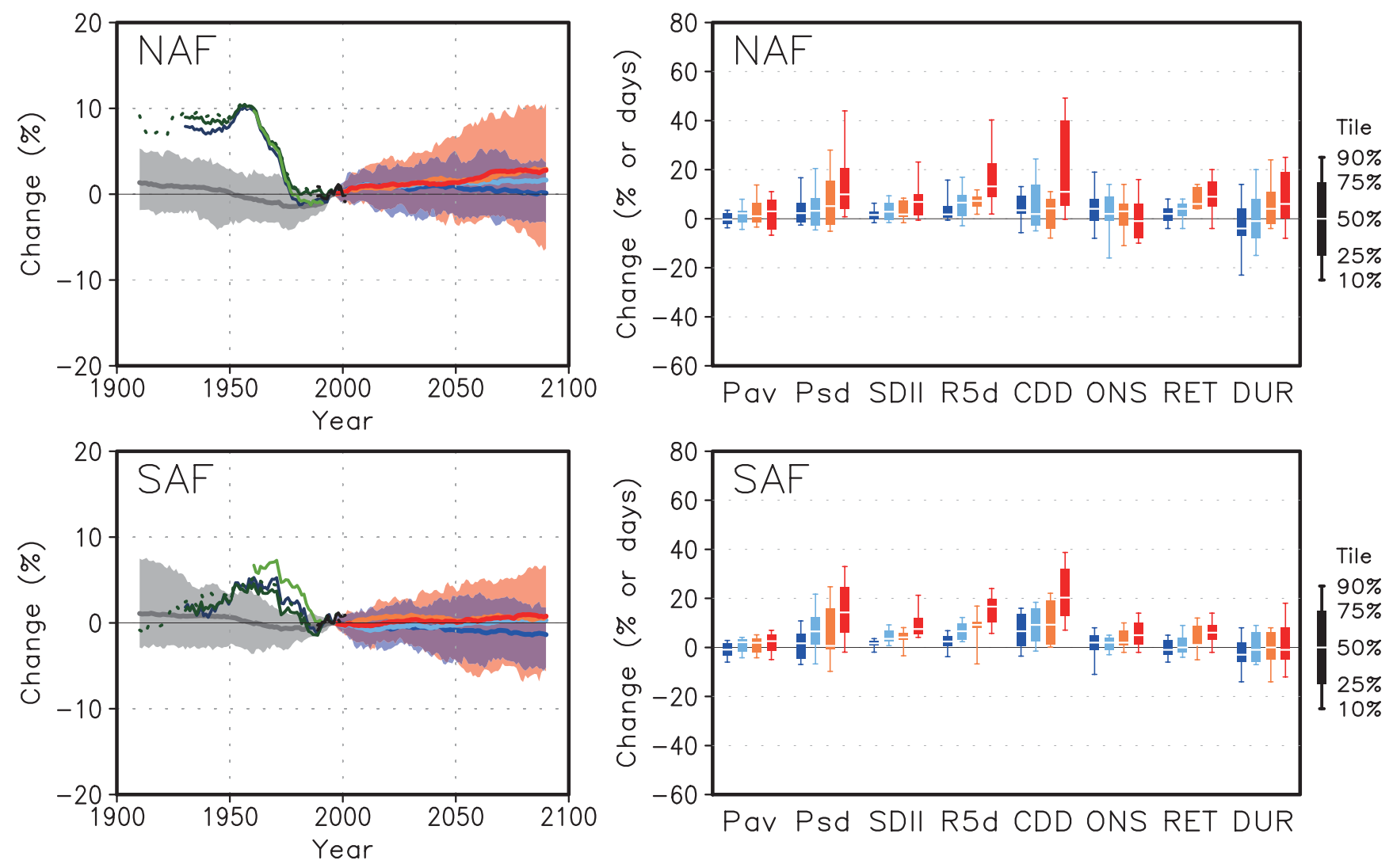

Figure 14.7 | As in Figure 14.4, except for (upper) North Africa (NAF) and (lower) South Africa (SAF).

confidence in precipitation associated with the NAMS will arrive later in the annual cycle, and persist longer. Projections of changes in the timing and duration of the SAMS remain uncertain. There is high confidence in the expansion of SAMS, resulting from increased temperature and humidity.

Based on how models represent known drivers of the West African monsoon, there is low confidence in projections of its future development based on CMIP5. Confidence is low in projections of a small delay in the onset of the West African rainy season with an intensification of late-season rains.

\subsection{Tropical Phenomena}

\subsubsection{Convergence Zones}

Section 7.6 presents a radiative perspective of changes in convection (including the differences between GHG and aerosol forcings), and Section 12.4.5.2 discusses patterns of precipitation change on the global scale. The emphasis here is on regional aspects of tropical changes. Tropical convection over the oceans, averaged for a month or longer, is organized into long and narrow convergence zones, often anchored by SST structures. Latent heat release in convection drives atmospheric circulation and affects global climate. In model experiments where spatially uniform SST warming is imposed, precipitation increases in these tropical convergence zones (Xie et al., 2010b), following the 'wet-get-wetter' paradigm (Held and Soden, 2006). On the flanks of a convergence zone, rainfall may decrease because of the increased horizontal gradient in specific humidity and the resultant increase in dry advection into the convergence zone (Neelin et al., 2003).

Although these arguments based on moist atmospheric dynamics call for changes in tropical convection to be organized around the climatological rain band, studies since AR4 show that such changes in a warmer climate also depend on the spatial pattern of SST warming. As a result of the SST pattern effect, rainfall change does not generally project onto the climatological convergence zones, especially for the annual mean. In CMIP3/5 model projections, annual rainfall change over tropical oceans follows a 'warmer-get-wetter' pattern, increasing where the SST warming exceeds the tropical mean and vice versa (Figure 14.8, Xie et al., 2010b; Sobel and Camargo, 2011; Chadwick et al., 2013). Differences among models in the SST warming pattern are an important source of uncertainty in rainfall projections, accounting for a third of inter-model variability in annual precipitation change in the tropics (Ma and Xie, 2013).

Figure 14.8 presents selected indices for several robust patterns of SST warming for RCP8.5. They include greater warming in the $\mathrm{NH}$ than in the Southern Hemisphere (SH), a pattern favouring rainfall increase at locations north of the equator and decreases to the south (Friedman et al., 2013); enhanced equatorial warming (Liu et al., 2005) that anchors a pronounced rainfall increase in the equatorial Pacific; reduced warming in the subtropical Southeast Pacific that weakens convection there; decreased zonal SST gradient across the equatorial Pacific (see Section 14.4) and increased westward SST gradient across the equatorial 
Indian Ocean (see Section 14.3.3) that together contribute to the weakened Walker cells.

Changes in tropical convection affect the pattern of SST change (Chou et al., 2005) and such atmospheric and oceanic perturbations are inherently coupled. The SST pattern effect dominates the annual rainfall change while the wet-get-wetter effect becomes important for seasonal mean rainfall in the summer hemisphere (Huang et al., 2013). This is equivalent to an increase in the annual range of precipitation in a warmer climate (Chou et al., 2013). Given uncertainties in SST warming pattern, the confidence is generally higher for seasonal than annual mean changes in tropical rainfall.

\subsubsection{Inter-Tropical Convergence Zone}

The Inter-Tropical Convergence Zone (ITCZ) is a zonal band of persistent low-level convergence, atmospheric convection, and heavy rainfall. Over the Atlantic and eastern half of the Pacific, the ITCZ is displaced north of the equator due to ocean-atmosphere interaction (Xie et al.,
2007) and extratropical influences (Kang et al., 2008; Fučkar et al., 2013). Many models show an unrealistic double-ITCZ pattern over the tropical Pacific and Atlantic, with excessive rainfall south of the equator (Section 9.4.2.5.1). This bias needs to be kept in mind in assessing ITCZ changes in model projections, especially for boreal spring when the model biases are largest.

The global zonal mean ITCZ migrates back and forth across the equator following the sun. In CMIP5, seasonal mean rainfall is projected to increase on the equatorward flank of the ITCZ (Figure 14.9). The co-migration of rainfall increase with the ITCZ is due to the wet-get-wetter effect while the equatorward displacement is due to the SST pattern effect (Huang et al., 2013).

\subsubsection{South Pacific Convergence Zone}

The South Pacific Convergence Zone (SPCZ, Widlansky et al., 2011) extends southeastward from the tropical western Pacific to French Polynesia and the SH mid-latitudes, contributing most of the yearly rainfall
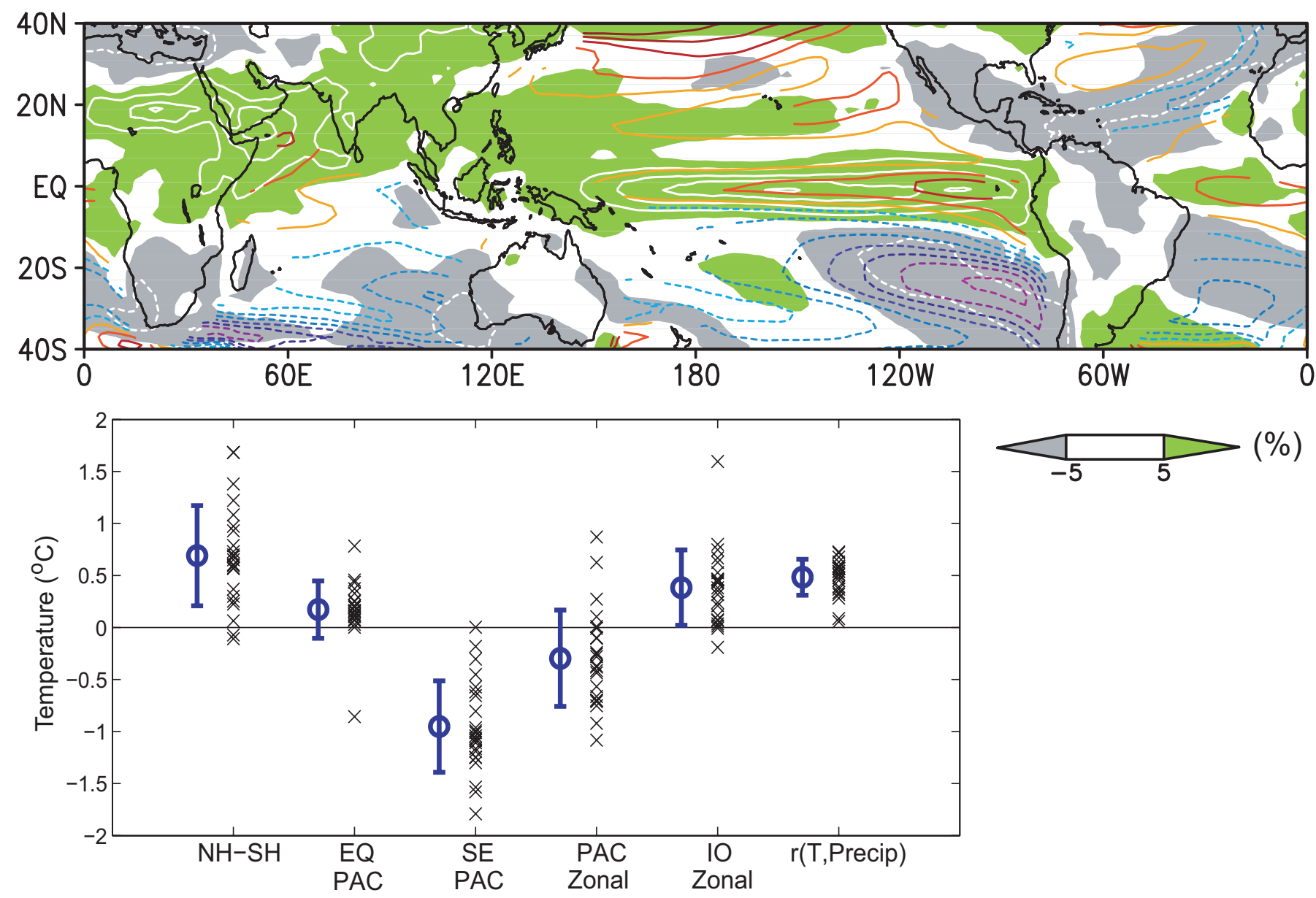

Figure 14.8 (Upper panel) Annual mean precipitation percentage change ( $\delta P / P$ in green/gray shade and white contours at 20\% intervals), and relative SST change (colour contours at intervals of $0.2^{\circ} \mathrm{C}$; negative dashed) to the tropical $\left(20^{\circ} \mathrm{S}\right.$ to $20^{\circ} \mathrm{N}$ ) mean warming in RCP8.5 projections, shown as $23 \mathrm{CMIP5}$ model ensemble mean. (Lower panel) Sea surface temperature (SST) warming pattern indices in the 23-model RCP8.5 ensemble, shown as the 2081-2100 minus $1986-2005$ difference. From left: Northern (EQ to $60^{\circ} \mathrm{N}$ ) minus Southern $\left(60^{\circ} \mathrm{S}\right.$ to EQ) Hemisphere; equatorial $\left(120^{\circ} \mathrm{E}\right.$ to $60^{\circ} \mathrm{W}, 5^{\circ} \mathrm{S}$ to $\left.5^{\circ} \mathrm{N}\right)$ and Southeast $\left(130^{\circ} \mathrm{W}\right.$ to $70^{\circ} \mathrm{W}, 30^{\circ} \mathrm{S}$ to $\left.15^{\circ} \mathrm{S}\right)$ Pacific relative to the tropical mean warming; zonal SST gradient in the equatorial Pacific $\left(120^{\circ} \mathrm{E}\right.$ to $180^{\circ} \mathrm{E}$ minus $150^{\circ} \mathrm{W}$ to $90^{\circ} \mathrm{W}, 5^{\circ} \mathrm{S}$ to $\left.5^{\circ} \mathrm{N}\right)$ and Indian $\left(50^{\circ} \mathrm{E}\right.$ to $70^{\circ} \mathrm{E}, 10^{\circ} \mathrm{S}$ to $10^{\circ} \mathrm{N}$ minus $90^{\circ} \mathrm{E}$ to $110^{\circ} \mathrm{S}, 10^{\circ} \mathrm{S}$ to $\left.\mathrm{EQ}\right) \mathrm{Oceans}^{\circ}$. (Rightmost) Spatial correlation ( $r$ ) between relative SST change and precipitation percentage change $(\delta P / P)$ in the tropics $\left(20^{\circ} S\right.$ to $\left.20^{\circ} \mathrm{N}\right)$ in each model. (The spatial correlation for the multi-model ensemble mean fields in the upper panel is 0.63 ). The circle and error bar indicate the ensemble mean and \pm 1 standard deviation, respectively. The upper panel is a CMIP5 update of Ma and Xie (2013), and see text for indices in the lower panel. 


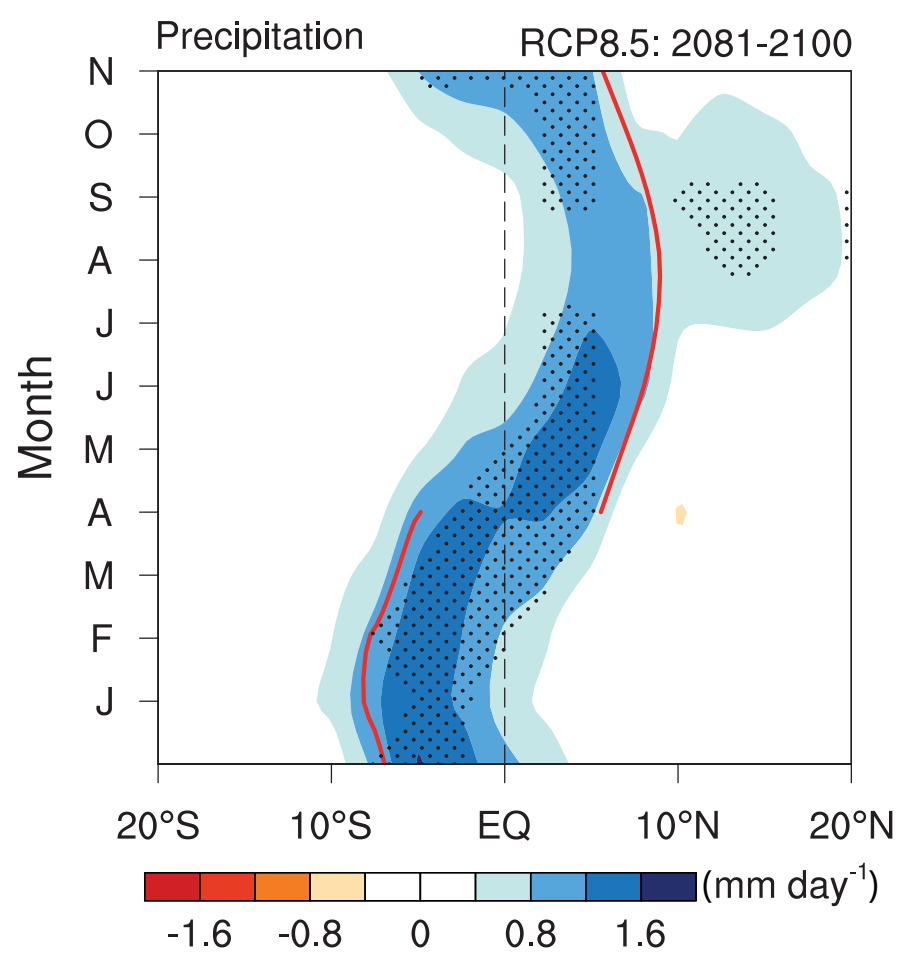

Figure 14.9 | Seasonal cycle of zonal mean tropical precipitation change (2081-2100 in RCP8.5 minus 1986-2005) in CMIP5 multi-model ensemble (MME) mean. Eighteen CMIP5 models were used. Stippling indicates that more than $90 \%$ models agree on the sign of MME change. The red curve represents the meridional maximum of the climatological rainfall. (Adapted from Huang et al., 2013.)

to the many South Pacific island nations under its influence. The SPCZ is most pronounced during austral summer (December, January and February (DJF)).

Zonal and meridional SST gradients, trade wind strength, and subsidence over the eastern Pacific are important mechanisms for SPCZ orientation and variability (Takahashi and Battisti, 2007; Lintner and Neelin, 2008; Vincent et al., 2011; Widlansky et al., 2011). Many GCMs simulate the SPCZ as lying east-west, giving a 'double-ITCZ' structure and missing the southeastward orientation (Brown et al., 2012a).

The majority of CMIP models simulate increased austral summer mean precipitation in the SPCZ, with decreased precipitation at the eastern edge of the SPCZ (Brown et al., 2012a; Brown et al., 2012b). The position of the SPCZ varies on interannual to decadal time scales, shifting northeast in response to El Niño (Folland et al., 2002; Vincent et al., 2011). Strong El Niño events induce a zonally oriented SPCZ located well northeast of its average position, while more moderate ENSO (Section 14.4) events are associated with movement of the SPCZ to the northeast or southwest, without a change in its orientation.

Models from both CMIP3 and CMIP5 that simulate the SPCZ well show a consistent tendency towards much more frequent zonally oriented SPCZ events in future (Cai et al., 2012b). The mechanism appears to be associated with a reduction in near-equatorial meridional SST gradient, a robust feature of modelled SST response to anthropogenic forcing (Widlansky et al., 2013). An increased frequency of zonally oriented
SPCZ events would have major implications for regional climate, possibly leading to longer dry spells in the southwest Pacific.

\subsubsection{South Atlantic Convergence Zone}

The South Atlantic Convergence Zone (SACZ) extends from the Amazon region through southeastern Brazil towards the Atlantic Ocean during austral summer (Cunningham and Cavalcanti, 2006; Carvalho et al., 2011; de Oliveira Vieira et al., 2013). Floods or dry conditions in southeastern Brazil are often related to SACZ variability (Muza et al., 2009; Lima et al., 2010; Vasconcellos and Cavalcanti, 2010). A subset of CMIP models simulate the SACZ (Vera and Silvestri, 2009; Seth et al., 2010; Yin et al., 2012) and its variability as a dipolar structure (Junquas et al., 2012; Cavalcanti and Shimizu, 2012).

A southward displacement of SACZ and intensification of the southern centre of the precipitation dipole are suggested in projections of CMIP3 and CMIP5 models (Seth et al., 2010; Junquas et al., 2012; Cavalcanti and Shimizu, 2012). This displacement is consistent with the increased precipitation over southeastern South America, south of $25^{\circ}$, projected for the second half of the 21st century, in CMIP3, CMIP5 and regional models (Figure Al.34, Figure 14.21). It is also consistent with the southward displacement of the Atlantic subtropical high (Seth et al., 2010) related to the southward expansion of the Hadley Cell (Lu et al., 2007). Pacific SST warming and the strengthening of the Pacific-South American (PSA)-like wave train (Section 14.6.2) are potential mechanisms for changes in the dipolar pattern resulting in SACZ change (Junquas et al., 2012). This change is also supported by the intensification and increased frequency of the low level jet over South America in future projections (Soares and Marengo, 2009; Seth et al., 2010).

\subsubsection{Madden-Julian Oscillation}

The MJO is the dominant mode of tropical intraseasonal (20 to 100 days) variability (Zhang, 2005). The MJO modulates tropical cyclone activity (Frank and Roundy, 2006), contributes to intraseasonal fluctuations of the monsoons (Maloney and Shaman, 2008), and excites teleconnection patterns outside the tropics (L'Heureux and Higgins, 2008; Lin et al., 2009). Simulation of the MJO by GCMs remains challenging, but with some improvements made in recent years (Section 9.5.2.3).

Possible changes in the MJO in a future warmer climate have just begun to be explored with models that simulate the phenomenon. In the Max Planck Institute Earth System Model, MJO variance increases appreciably with increasing warming (Schubert et al., 2013). The change in MJO variance is highly sensitive to the spatial pattern of SST warming (Maloney and Xie, 2013). In light of the low skill in simulating MJO, and its sensitive to SST warming pattern, which in itself is subject to large uncertainties, it is currently not possible to assess how the MJO will change in a warmer climate.

\subsubsection{Indian Ocean Modes}

The tropical Indian Ocean SST exhibits two modes of interannual variability (Schott et al., 2009; Deser et al., 2010b): the Indian Ocean Basin (IOB) mode featuring a basin-wide structure of the same sign, and the Indian Ocean Dipole (IOD) mode with largest amplitude in the eastern 
Indian Ocean off Indonesia, and weaker anomalies of the opposite polarity over the rest of the basin (Box 2.5). Both modes are statistically significantly correlated with ENSO (Section 14.4). CMIP models simulate both modes well (Section 9.5.3.4.2).

The formation of IOB is linked to ENSO via an atmospheric bridge and surface heat flux adjustment (Klein et al., 1999; Alexander et al., 2002). Ocean-atmosphere interactions within the Indian Ocean are important for the long persistence of this mode (Izumo et al., 2008; Wu et al., 2008; Du et al., 2009). The basin mode affects the termination of ENSO events (Kug and Kang, 2006), it induces coherent atmospheric anomalies in the summer following El Niño (Xie et al., 2009), including supressed convection (Wang et al., 2003) and reduced tropical cyclone activity (Du et al., 2011) over the Northwest Pacific and anomalous rainfall over East Asia (Huang et al., 2004).

IOD develops in July to November and involves Bjerknes feedback between zonal SST gradient, zonal wind and thermocline tilt along the equator (Saji et al., 1999; Webster et al., 1999). A positive IOD event (with negative SST anomalies off Sumatra) is associated with droughts in Indonesia, reduced rainfall over Australia, intensified Indian summer monsoon, increased precipitation in East Africa and anomalous conditions in the extratropical SH (Yamagata et al., 2004). Most CMIP3 models are able to reproduce the general features of the IOD, including its phase lock onto the July to November season, while detailed analysis of CMIP5 simulations are not yet available (Section 9.5.3.4.2)

Basin-mean SST has risen steadily for much of the 20th century, a trend captured by CMIP3 20th century simulations (Alory et al., 2007). The SST increase over the North Indian Ocean since about 1930 is noticeably weaker than for the rest of the basin. This spatial pattern is suggestive of the effects of reduced surface solar radiation due to Asian brown clouds (Chung and Ramanathan, 2006) and it affects Arabian

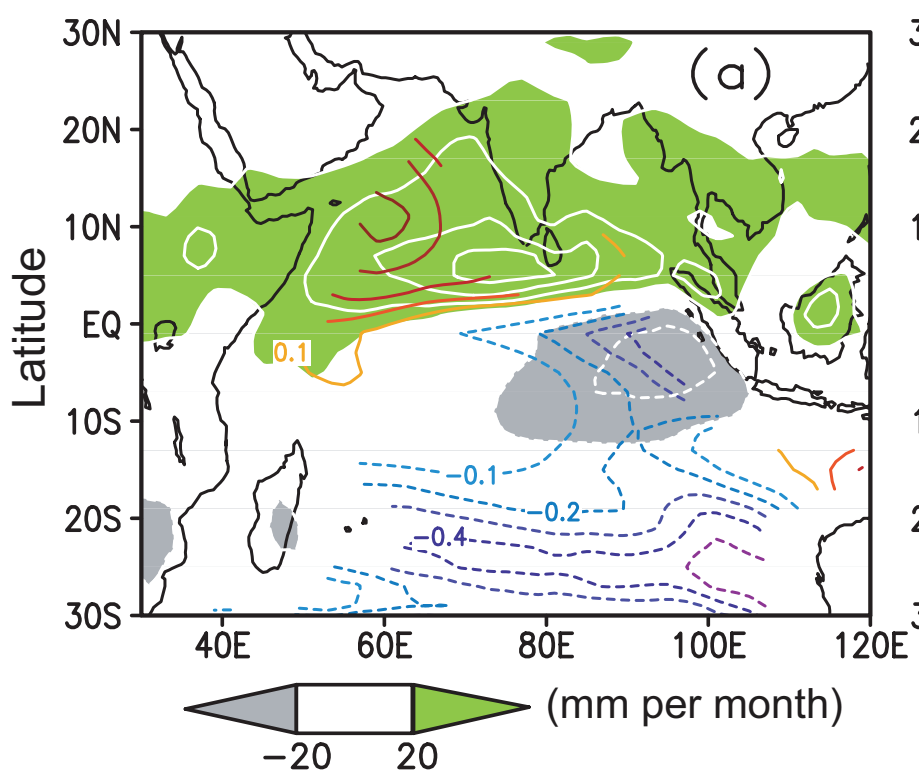

Sea cyclones (Evan et al., 2011b). In the equatorial Indian Ocean, coral isotope records off Indonesia indicate a reduced SST warming and/or increased salinity during the 20th century (Abram et al., 2008). From ship-borne surface measurements, an easterly wind change especially during July to October has been observed over the past six decades, a result consistent with a reduction of marine cloudiness in the east and a decreasing precipitation trend over the maritime continent (Tokinaga et al., 2012). Atmospheric reanalysis products have difficulty representing these changes (Han et al., 2010).

The projected changes over the equatorial Indian Ocean include easterly wind anomalies, a shoaling thermocline (Vecchi and Soden, 2007a; Du and Xie, 2008) and reduced SST warming in the east (Stowasser et al., 2009), a result confirmed by CMIP5 multi-model analysis (Zheng et al., 2013; Figure 14.10). The change in zonal SST gradient, in turn, reinforces the easterly wind change, indicative of a positive feedback between them as envisioned by Bjerknes (1969). This coupled pattern is most pronounced during July to November, and is broadly consistent with the observed changes in the equatorial Indian Ocean.

In one CMIP3 model, the IOB mode and its capacitor effect persist longer, through summer into early fall towards the end of the century (2081-2100, Zheng et al., 2011). This increased persistence intensifies ENSO's influence on the Northwest Pacific summer monsoon. The confidence level of this relationship is low due to the lack of multi-model studies.

The IOD variability in SST remains nearly unchanged in future projections of CMIP3 and CMIP5 (Ihara et al., 2009; Figure 14.11a) despite the easterly wind change that lifts the thermocline (Figure 14.10b) and intensifies thermocline feedback on SST in the eastern equatorial Indian Ocean. The global increase in atmospheric dry static stability weakens the atmospheric response to zonal SST gradient changes,

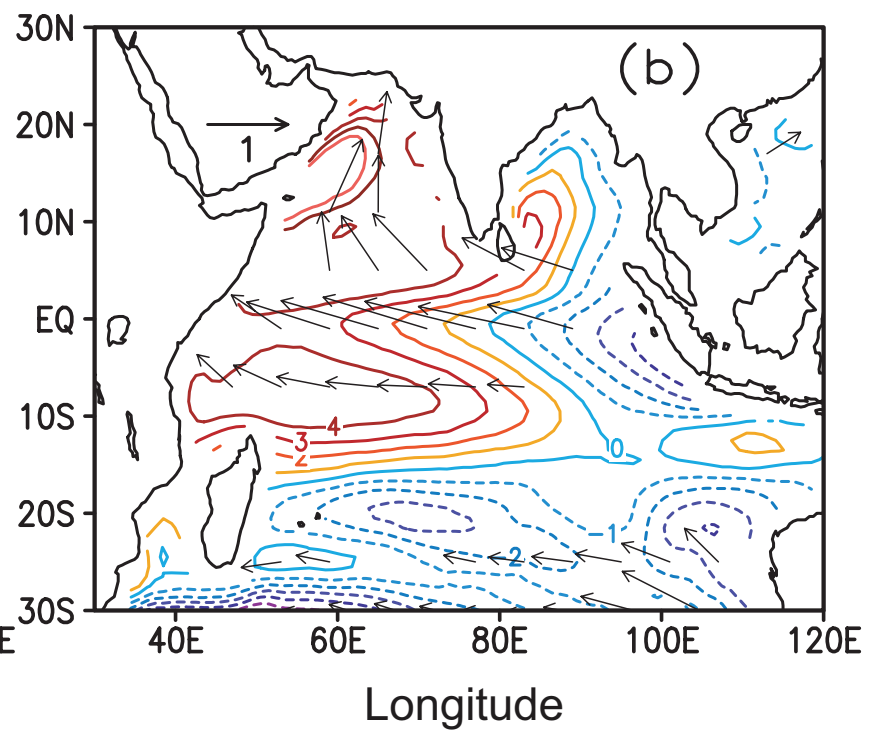

Figure 14.10 | September to November changes in a 22-model CMIP5 ensemble (2081-2100 in RCP8.5 minus 1986-2005 in historical run). (a) Sea surface temperature (SST, colour contours at $0.1^{\circ} \mathrm{C}$ intervals) relative to the tropical mean $\left(20^{\circ} \mathrm{S}\right.$ to $20^{\circ} \mathrm{N}$ ), and precipitation (shading and white contours at $20 \mathrm{~mm}$ per month intervals). (b) Surface wind velocity $\left(\mathrm{m} \mathrm{s}^{-1}\right)$, and sea surface height deviation from the global mean (contours, centimetres). Over the equatorial Indian Ocean, ocean-atmospheric changes form Bjerknes feedback, with the reduced SST warming and suppressed convection in the east. (Updated with CMIP5 from Xie et al., 2010b.) 


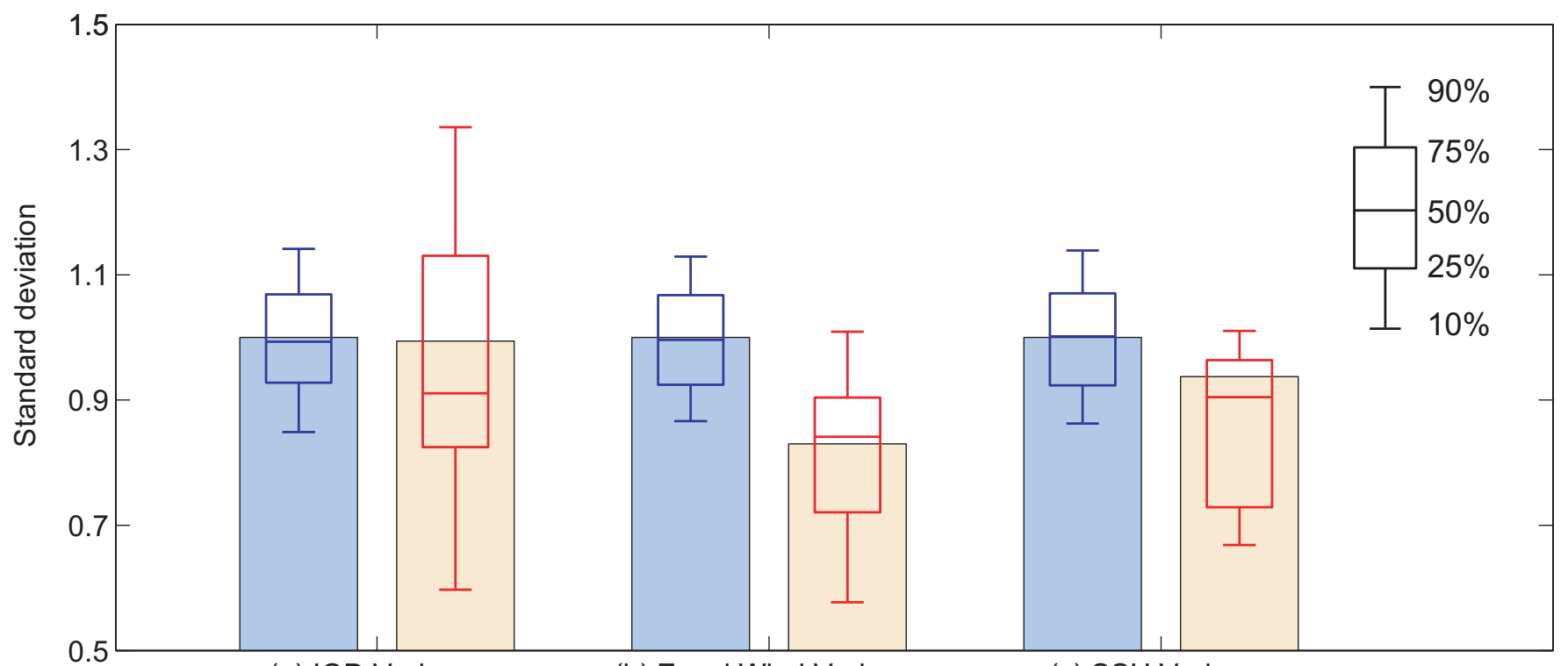

(a) IOD Variance

(b) Zonal Wind Variance

(c) SSH Variance

Figure 14.11 | CMIP5 multi-model ensemble mean standard deviations of interannual variability for September to November in pre-industrial (PiControl; blue bars) and RCP8.5 (red) runs: (a) the Indian Ocean dipole index defined as the western $\left(50^{\circ} \mathrm{E}\right.$ to $70^{\circ} \mathrm{E}, 10^{\circ} \mathrm{S}$ to $\left.10^{\circ} \mathrm{N}\right)$ minus eastern $\left(90^{\circ} \mathrm{E}\right.$ to $110^{\circ} \mathrm{E}, 10^{\circ} \mathrm{S}$ to $\left.0^{\circ}\right) \mathrm{SST}$ difference; (b) zonal wind in the central equatorial Indian Ocean $\left(70^{\circ} \mathrm{E}\right.$ to $90^{\circ} \mathrm{E}, 5^{\circ} \mathrm{S}$ to $\left.5^{\circ} \mathrm{N}\right)$; and (c) sea surface height in the eastern equatorial Indian Ocean $\left(90^{\circ} \mathrm{E}\right.$ to $110^{\circ} \mathrm{E}, 10^{\circ} \mathrm{S}$ to $\left.0^{\circ}\right)$. The standard deviation is normalized by the pre-industrial (PiControl) value for each model before ensemble average. Blue box-and-whisker plots show the 10th, 25th, 50th, 75th and 90th percentiles of 51-year windows for PiControl, representing natural variability. Red box-and-whisker plots represent inter-model variability for RCP8.5, based on the nearest rank. (Adapted from Zheng et al., 2013.)

countering the enhanced thermocline feedback (Zheng et al., 2010). The weakened atmospheric feedback is reflected in a decrease in IOD variance in both zonal wind and the thermocline depth (Zheng et al., 2013; Figure 14.11b, c).

\subsubsection{Atlantic Ocean Modes}

The Atlantic features a northward-displaced ITCZ (Section 14.3.1.1), and a cold tongue that develops in boreal summer. Climate models generally fail to simulate these characteristics of tropical Atlantic climate (Section 9.5.3.3). The biases severely limit model skill in simulating modes of Atlantic climate variability and in projecting future climate change in the Atlantic sector. In-depth analysis of the CMIP5 projections of Atlantic Ocean Modes has not yet been fully explored, but see Section 12.4.3.

The inter-hemispheric SST gradient displays pronounced interannual to decadal variability (Box 2.5, Figure 2), referred to as the Atlantic meridional mode (AMM; Servain et al., 1999; Chiang and Vimont, 2004; Xie and Carton, 2004). A thermodynamic feedback between surface winds, evaporation and SST (WES; Xie and Philander, 1994) is fundamental to the AMM (Chang et al., 2006). This mode affects precipitation in northeastern Brazil by displacing the ITCZ (Servain et al., 1999; Chiang and Vimont, 2004; Xie and Carton, 2004), and Atlantic hurricane activity (Vimont and Kossin, 2007; Smirnov and Vimont, 2011).

The Atlantic Niño mode represents interannual variability in the equatorial cold tongue, akin to ENSO (Box 2.5, Figure 2). Bjerknes feedback is considered important for energizing the mode (Zebiak, 1993; Carton and Huang, 1994; Keenlyside and Latif, 2007). This mode affects the West Africa Monsoon (Vizy and Cook, 2002; Giannini et al., 2003).
Over the past century, the Atlantic has experienced a pronounced and persistent warming trend. The warming has brought detectable changes in atmospheric circulation and rainfall patterns in the region. In particular, the ITCZ has shifted southward and land precipitation has increased over the equatorial Amazon, equatorial West Africa, and along the Guinea coast, while it has decreased over the Sahel (Deser et al., 2010a; Tokinaga and Xie, 2011; see also Sections 2.5 and 2.7 ). Atlantic Niño variability has weakened by $40 \%$ in amplitude from 1960 to 1999 , associated with a weakening of the equatorial cold tongue (Tokinaga and Xie, 2011).

The CMIP3 20th century climate simulations generally capture the warming trend of the basin-averaged SST over the tropical Atlantic. A majority of the models also seem to capture the secular trend in the tropical Atlantic SST inter-hemispheric gradient and, as a result, the southward shift of the Atlantic ITCZ over the past century (Chang et al., 2011).

Many CMIP3 model simulations with the A1B emission scenario show only minor changes in the SST variance associated with the AMM. However, the few models that give the best AMM simulation over the 20th century project a weakening in future AMM activity (Breugem et al., 2006), possibly due to the northward shift of the ITCZ (Breugem et al., 2007). At present, model projections of future change in AMM activity is considered highly uncertain because of the poorly simulated Atlantic ITCZ. In fact, uncertainty in projected changes in Atlantic meridional SST gradient limits the confidence in regional climate projections surrounding the tropical Atlantic Ocean (Good et al., 2008).

A majority of CMIP3 models forced with the A1B emission scenario project no major change in Atlantic Niño activity in the 21st century, 
while a few models project a sizable decrease in future activity (Breugem et al., 2006).

CMIP5 projections show an accelerated SST warming over much of the tropical Atlantic (Figure 12.11). RCP8.5 projections of the inter-hemispheric SST gradient change within the basin, however, are not consistent among CMIP5 models as future GHG increase dominates over the anthropogenic aerosol effect.

\subsubsection{Assessment Summary}

There is medium confidence that annual rainfall changes over tropical oceans follow a 'warmer-get-wetter' pattern, increasing where the SST warming exceeds the tropical mean and vice versa. One third of inter-model differences in precipitation projection are due to those in SST pattern. The SST pattern effect on precipitation change is a new finding since AR4.

The wet-get-wetter effect is more obvious in the seasonal than annual rainfall change in the tropics. Confidence is generally higher in seasonal than in annual mean changes in tropical precipitation. There is medium confidence that seasonal rainfall will increase on the equatorward flank of the current ITCZ; that the frequency of zonally oriented SPCZ events will increase, with the SPCZ lying well to the northeast of its average position during those events; and that the SACZ shifts southwards, in conjunction with the southward displacement of the South Atlantic subtropical high, leading to an increase in precipitation over southeastern South America.

Owing to models' ability to reproduce general features of IOD and agreement on future projections, it is likely that the tropical Indian Ocean will feature a zonal pattern with reduced (enhanced) warming and decreased (increased) rainfall in the east (west), a pattern especially pronounced during August to November. The Indian Ocean dipole mode will very likely remain active, with interannual variability unchanged in SST but decreasing in thermocline depth. There is low confidence in changes in the summer persistence of the Indian Ocean SST response to ENSO and in ENSO's influence on summer climate over the Northwest Pacific and East Asia.

The observed SST warming in the tropical Atlantic represents a reduction in spatial variation in climatology: the warming is weaker north than south of the equator; and the equatorial cold tongue weakens both in the mean and interannual variability. There is low confidence in projected changes over the tropical Atlantic, both for the mean and interannual modes, because of large errors in model simulations of current climate.

There is low confidence in how MJO will change in the future due to the poor skill of models in simulating MJO and the sensitivity of its change to SST warming patterns that are themselves subject to large uncertainties in the projections.

\subsection{El Niño-Southern Oscillation}

The ENSO is a coupled ocean-atmosphere phenomenon naturally occurring at the interannual time scale over the tropical Pacific (see Box 2.5, Supplementary Material Section 14.SM.2, and Figure 14.12).

\subsubsection{Tropical Pacific Mean State}

SST in the western tropical Pacific has increased by up to $1.5^{\circ} \mathrm{C}$ per century, and the warm pool has expanded (Liu and Huang, 2000; Huang and Liu, 2001; Cravatte et al., 2009). Studies disagree on how the eastwest SST gradient along the equator has changed, some showing a strengthening (Cane et al., 1997; Hansen et al., 2006; Karnauskas et al., 2009; An et al., 2011) and others showing a weakening (Deser et al., 2010a; Tokinaga et al., 2012), because of observational uncertainties associated with limited data sampling, changing measurement techniques, and analysis procedures. Most CMIP3 and CMIP5 models also disagree on the response of zonal SST gradient across the equatorial Pacific (Yeh et al., 2012).

The Pacific Ocean warms more near the equator than in the subtropics in CMIP3 and CMIP5 projections (Liu et al., 2005; Gastineau and Soden, 2009; Widlansky et al., 2013; Figure 14.12) because of the difference in evaporative damping (Xie et al., 2010b). Other oceanic changes include a basin-wide thermocline shoaling (Vecchi and Soden, 2007a; DiNezio et al., 2009; Collins et al., 2010; Figure 14.12), a weakening of surface currents, and a slight upward shift and strengthening of the equatorial undercurrent (Luo and Rothstein, 2011; Sen Gupta et al., 2012). A weakening of tropical atmosphere circulation during the 20th century was documented in observations and reanalyses (Vecchi et al., 2006; Zhang and Song, 2006; Vecchi and Soden, 2007a; Bunge and Clarke, 2009; Karnauskas et al., 2009; Yu and Zwiers, 2010; Tokinaga et al., 2012) and in CMIP models (Vecchi and Soden, 2007a; Gastineau and Soden, 2009). The Pacific Walker Circulation, however, intensified during the most recent two decades (Mitas and Clement, 2005; Liu and Curry, 2006; Mitas and Clement, 2006; Sohn and Park, 2010; Li and Ren, 2012; Zahn and Allan, 2011; Zhang et al., 2011a), illustrating the effects of natural variability.

\subsubsection{El Niño Changes over Recent Decades and in the Future}

The amplitude modulation of ENSO at longer time scales has been observed in reconstructed instrumental records (Gu and Philander, 1995; Wang, 1995; Mitchell and Wallace, 1996; Wang and Wang, 1996; Power et al., 1999; An and Wang, 2000; Yeh and Kirtman, 2005; Power and Smith, 2007; Section 5.4.1), in proxy records (Cobb et al., 2003; Braganza et al., 2009; Li et al., 2011c; Yan et al., 2011), and is also simulated by coupled GCMs (Lau et al., 2008; Wittenberg, 2009). Some studies have suggested that the modulation was due to changes in mean climate conditions in the tropical Pacific (An and Wang, 2000; Fedorov and Philander, 2000; Wang and An, 2001, 2002; Li et al., 2011c), as observed since the 1980s (An and Jin, 2000; An and Wang, 2000; Fedorov and Philander, 2000; Kim and An, 2011). With three events during 2000-2010, which meets intensity in Nino4 being larger than in Nino3, two events during 1990-2000 and only two events are found for 1950-1990 the maximum SST warming during El Niño now 


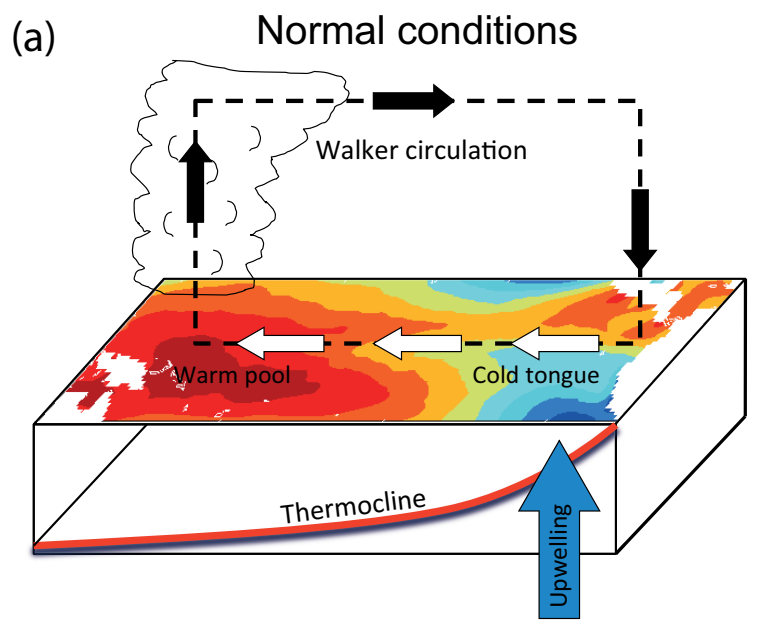

(b)

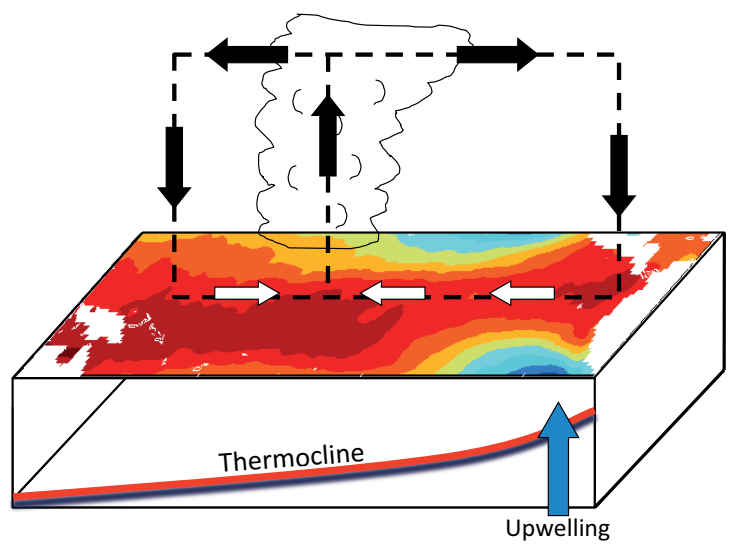

(c)

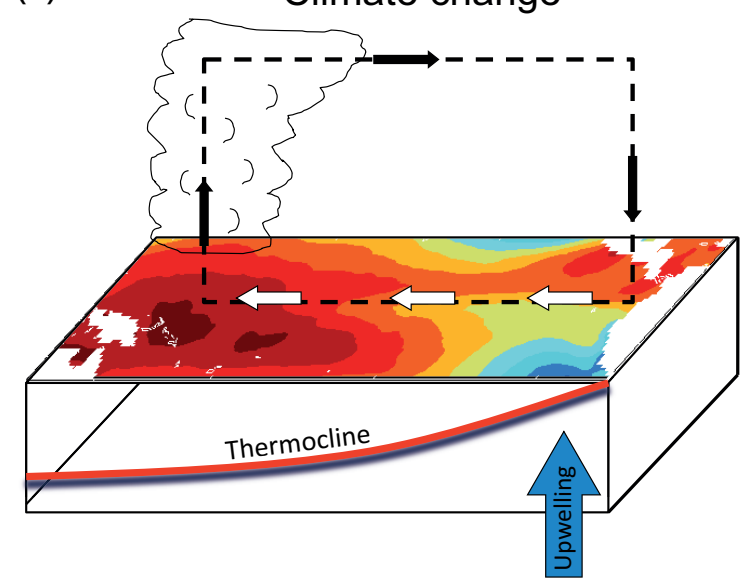

El Niño Conditions (SST anomalies)

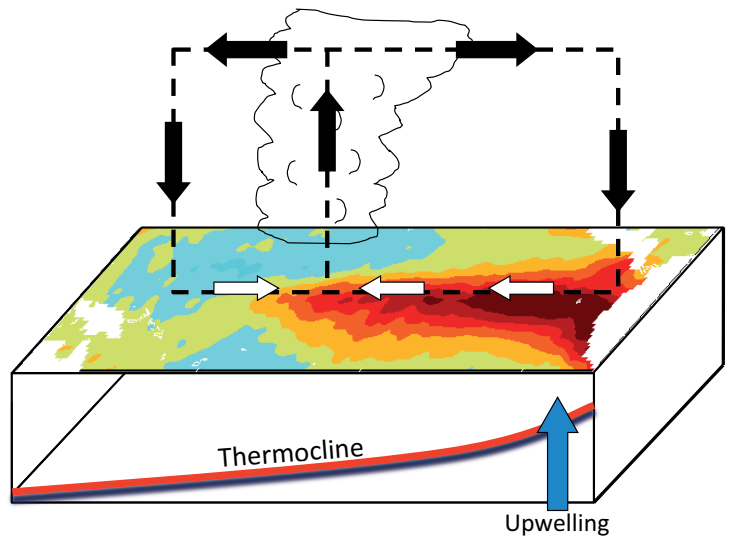

\section{Climate change (SST anomalies)}

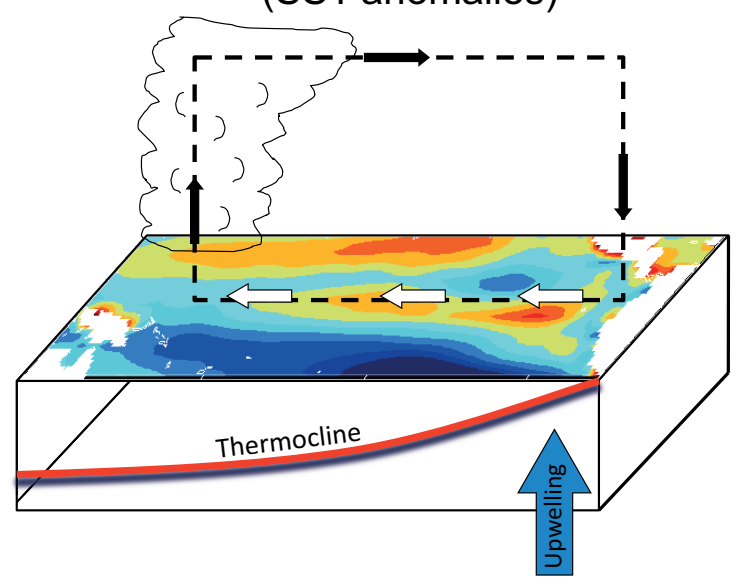

Figure 14.12 | Idealized schematic showing atmospheric and oceanic conditions of the tropical Pacific region and their interactions during normal conditions, El Niño conditions, and in a warmer world. (a) Mean climate conditions in the tropical Pacific, indicating sea surface temperatures (SSTs), surface wind stress and associated Walker Circulation, the mean position of convection and the mean upwelling and position of the thermocline. (b) Typical conditions during an El Niño event. SSTs are anomalously warm in the east; convection moves into the central Pacific; the trade winds weaken in the east and the Walker Circulation is disrupted; the thermocline flattens and the upwelling is reduced. (c) The likely mean conditions under climate change derived from observations, theory and coupled General Circulation Models (GCMs). The trade winds weaken; the thermocline flattens and shoals; the upwelling is reduced although the mean vertical temperature gradient is increased; and SSTs (shown as anomalies with respect to the mean tropical-wide warming) increase more on the equator than off. Diagrams with absolute SST fields are shown on the left, diagrams with SST anomalies are shown on the right. For the climate change fields, anomalies are expressed with respect to the basin average temperature change so that blue colours indicate a warming smaller than the basin mean, not a cooling (Collins et al., 2010). 


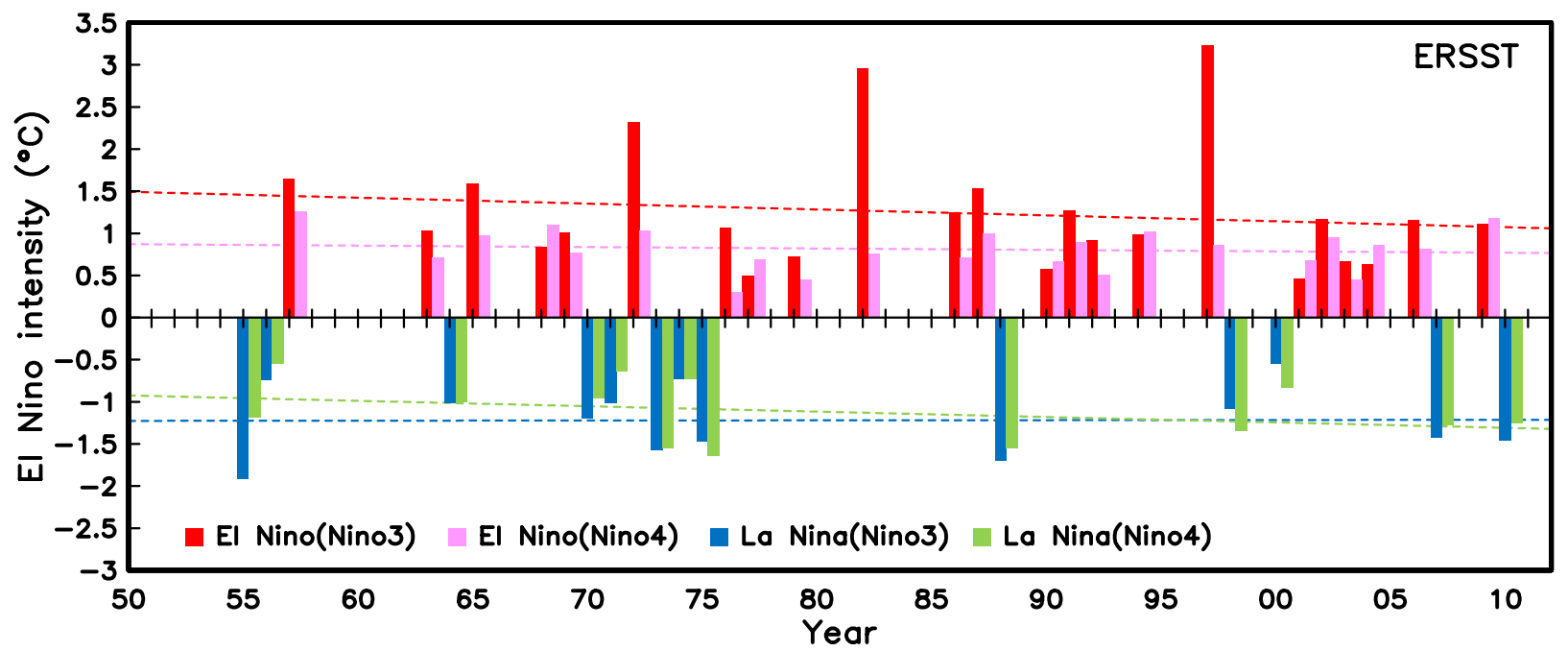

Figure 14.13 Intensities of El Niño and La Niña events for the last 60 years in the eastern equatorial Pacific (Niño3 region) and in the central equatorial Pacific (Niño4 region), and the estimated linear trends, obtained from Extended Reconstructed Sea Surface Temperature v3 (ERSSTv3).

appears to occur more often in the central Pacific (Figure 14.13; Ashok et al., 2007; Kao and Yu, 2009; Kug et al., 2009; Section 9.5.3.4.1 and Supplementary Material Section 14.SM.2; Yeh et al., 2009), with global impacts that are distinct from 'standard' El Niño events where the maximum warming is over the eastern Pacific (Kumar et al., 2006a; Ashok et al., 2007; Kao and Yu, 2009; Hu et al., 2012b). During the past century, an increasing trend in ENSO amplitude was also observed (Li et al., 2011c; Vance et al., 2012), possibly caused by a warming climate (Zhang et al., 2008; Kim and An, 2011) although other reconstructions in this data-sparse region dispute this trend (Giese and Ray, 2011).

Long coupled GCM simulations show that decadal-to-centennial modulations of ENSO can be generated without any change in external forcing (Wittenberg, 2009; Yeh et al., 2011), with multi-decadal epochs of anomalous ENSO behaviour. The modulations result from nonlinear processes in the tropical climate system (Timmermann et al., 2003), the interaction with the mean climate state (Ye and Hsieh, 2008; Choi et al., 2009,2011 , 2012), or from random changes in ENSO activity triggered by chaotic atmospheric variability (Power and Colman, 2006; Power et al., 2006). There is little consensus as to whether the decadal modulations of ENSO properties (amplitude and spatial pattern) during recent decades are due to anthropogenic effects or natural variability. Instrumental SST records are available back to the 1850s, but good observations of the coupled air-sea feedbacks that control ENSO behaviourincluding subsurface temperature and current fluctuations, and air-sea exchanges of heat, momentum and water-are available only after the late 1970s, making observed historical variations in ENSO feedbacks highly uncertain (Chen, 2003; Wittenberg, 2004).

CMIP5 models show some improvement compared to CMIP3, especially in ENSO amplitude (Section 9.5.3.4.1). Selected CMIP5 models that simulate well strong El Niño events show a gradual increase of EI Niño intensity, especially over the central Pacific (Kim and Yu, 2012). CMIP3 models suggested a westward shift of SST variability in future projections (Boer, 2009; Yeh et al., 2009). Generally, however, future changes in El Niño intensity in CMIP5 models are model dependent (Guilyardi et al., 2012; Kim and Yu, 2012; Stevenson et al., 2012), and not significantly distinguished from natural modulations (Stevenson, 2012; Figure 14.14). Because the change in tropical mean conditions (especially the zonal gradient) in a warming climate is model dependent (Section 14.4.1), changes in ENSO intensity for the 21st century (Solomon and Newman, 2011; Hu et al., 2012a) are uncertain (Figure 14.14). Future changes in ENSO depend on competing changes in coupled ocean-atmospheric feedback (Philip and Van Oldenborgh, 2006; Collins et al., 2010; Vecchi and Wittenberg, 2010), and on the dynamical regime a given model is in. There is high confidence, however, that ENSO will remain the dominant mode of natural climate variability in the 21st century (Collins et al., 2010; Guilyardi et al. 2012; Kim and Yu 2012; Stevenson 2012).

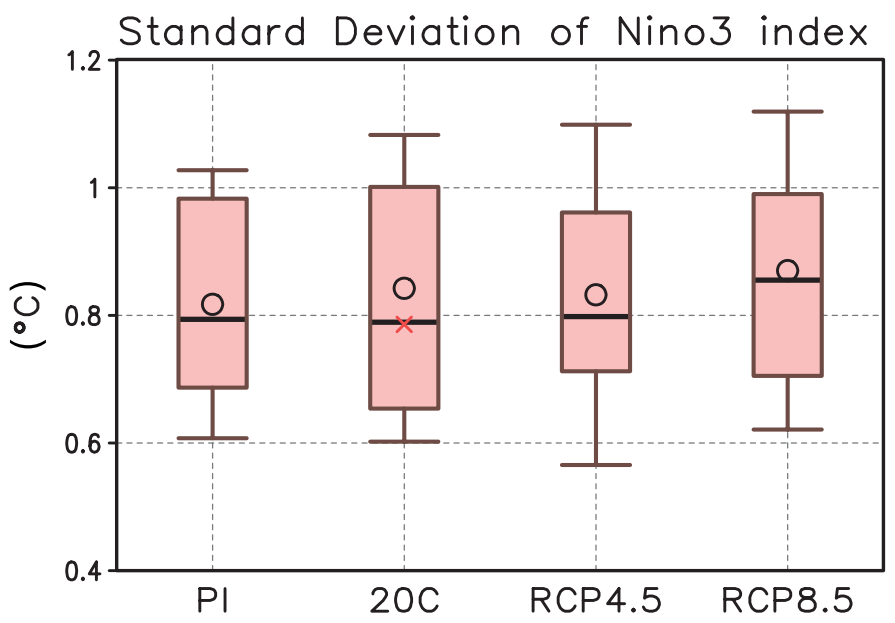

Figure 14.14 | Standard deviation in CMIP5 multi-model ensembles of sea surface temperature variability over the eastern equatorial Pacific Ocean (Nino3 region: $5^{\circ} \mathrm{S}$ $5^{\circ} \mathrm{N}, 150^{\circ} \mathrm{W}-90^{\circ} \mathrm{W}$ ), a measure of El Nino amplitude, for the pre-industrial (PI) control and 20th century (20C) simulations, and 21st century projections using RCP4.5 and RCP8.5. Thirty-one models are used for the ensemble average. Open circles indicate multi-model ensemble means, and the red cross symbol is the observed standard deviation for January 1870 - December 2011 obtained from HadISSTv1. The linear trend and climatological mean of seasonal cycle have been removed. Box-whisker plots show the 16th, 25th, 50th, 75th, and 84th percentiles. 


\subsubsection{Teleconnections}

There is little improvement in the CMIP5 ensemble relative to CMIP3 in the amplitude and spatial correlation metrics of precipitation teleconnections in response to ENSO, in particular within regions of strong observed precipitation teleconnections (equatorial South America, the western equatorial Pacific and a southern section of North America; Langenbrunner and Neelin, 2013). Scenario projections in CMIP3 and CMIP5 showed a systematic eastward shift in both El Niño- and La Niña-induced teleconnection patterns over the extratropical NH (Meehl and Teng, 2007; Stevenson et al., 2012; Figure 14.15), which might be due to the eastward migration of tropical convection centres associated with the expansion of the warm pool in a warm climate (Muller and Roeckner, 2006; Müller and Roeckner, 2008; Cravatte et al., 2009; Kug et al., 2010), or changes in the mid-latitude mean circulation (Meehl and Teng, 2007). Some models produced an intensified ENSO teleconnection pattern over the North Atlantic region in a warmer climate (Müller and Roeckner, 2008; Bulic et al., 2012) and a weakened teleconnection pattern over the North Pacific (Stevenson, 2012). It is unclear whether the eastward shift of tropical convection is related to longitudinal shifts in El Niño maximum SST anomalies (see Supplementary Material Section 14.SM.2) or to changes in the mean state in the tropical Pacific. Some coupled GCMs, which do not show an increase in the central Pacific warming during El Nino in response to a warming climate, do not produce a substantial change in the longitudinal location of tropical convection (Müller and Roeckner, 2008; Yeh et al., 2009).

In a warmer climate, the increase in atmospheric moisture intensifies temporal variability of precipitation even if atmospheric circulation variability remains the same (Trenberth 2011; Section 12.4.5). This applies to ENSO-induced precipitation variability but the possibility of changes in ENSO teleconnections complicates this general conclusion, making it somewhat regional-dependent (Seager et al. 2012)

\subsubsection{Assessment Summary}

ENSO shows considerable inter-decadal modulations in amplitude and spatial pattern within the instrumental record. Models without changes in external forcing display similar modulations, and there is little consensus on whether the observed changes in ENSO are due to external forcing or natural variability (see also Section 10.3.3 for an attribution discussion).

There is high confidence that ENSO will remain the dominant mode of interannual variability with global influences in the 21st century, and due to changes in moisture availability ENSO-induced rainfall variability on regional scales will intensify. There is medium confidence that ENSO-induced teleconnection patterns will shift eastward over the North Pacific and North America. There is low confidence in changes in the intensity and spatial pattern of El Niño in a warmer climate.

\subsection{Annular and Dipolar Modes}

The North Atlantic Oscillation (NAO), the North Pacific Oscillation (NPO) and the Northern and Southern Annular Modes (NAM and SAM) are dominant modes of variability in the extratropics. These modes are the focus of much research attention, especially in impact studies, where they are often used as aggregate descriptors of past regional climate
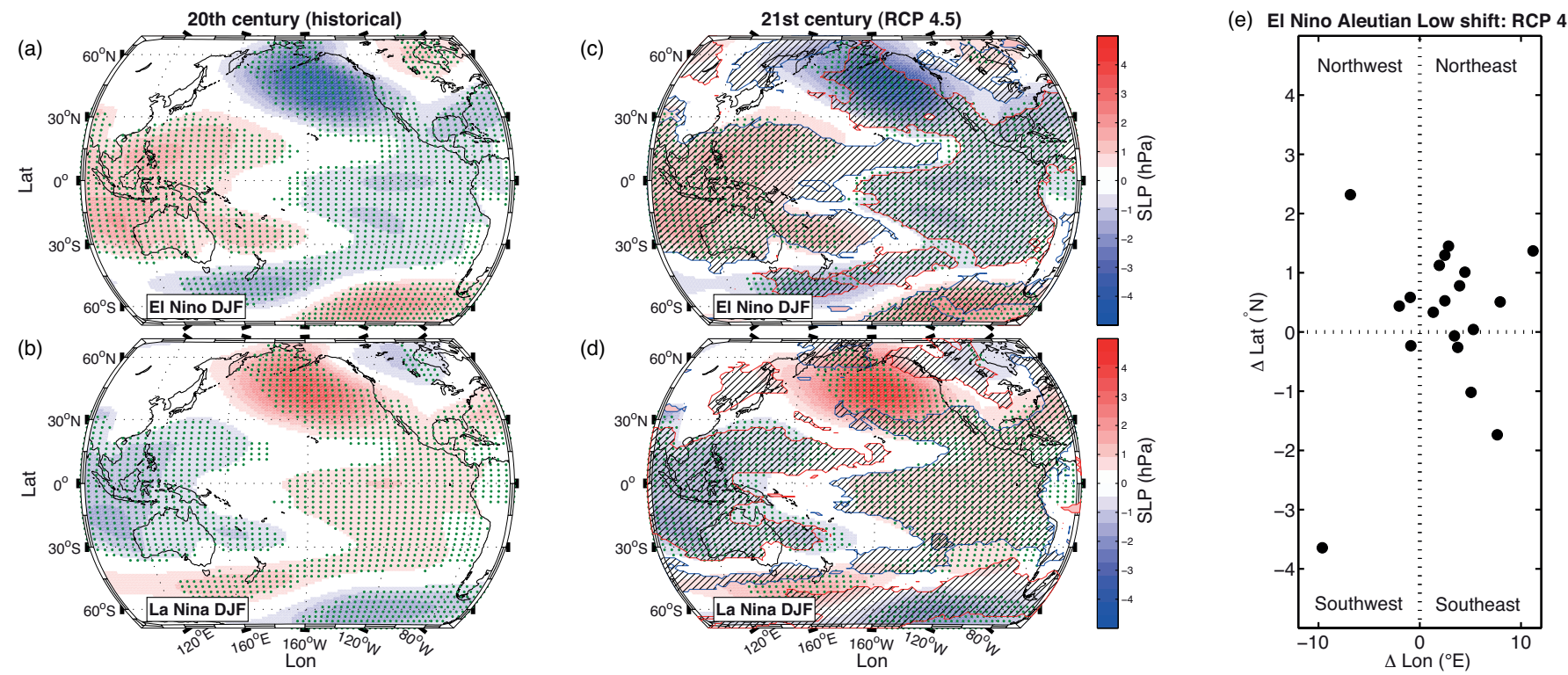

Figure 14.15 | Changes to sea level pressure (SLP) teleconnections during December, January and February (DJF) in the CMIP5 models. (a) SLP anomalies for El Niño during the 20th century. (b) SLP anomalies for La Nina during the 20th century. (c) SLP anomalies for El Niño during RCP4.5. (d) SLP anomalies for La Niña during RCP4.5. Maps in (a)-(d) are stippled where more than two thirds of models agree on the sign of the SLP anomaly ((a), ( b): 18 models; (c), (d): 12 models), and hatched where differences between the RCP4.5 multi-model mean SLP anomaly exceed the 60th percentile (red-bordered regions) or are less than the 40th percentile (blue-bordered regions) of the distribution of 20th century ensemble means. In all panels, El Niño (La Niña) periods are defined as years having DJF Nino3.4 SST above (below) one standard deviation relative to the mean of the detrended time series. For ensemble mean calculations, all SLP anomalies have been normalized to the standard deviation of the ensemblemember detrended Nino3.4 SST. (e) Change in the 'centre of mass' of the Aleutian Low SLP anomaly, RCP4.5-20th century. The Aleutian Low SLP centre of mass is a vector with two elements (lat, lon), and is defined as the sum of (lat, lon) weighted by the SLP anomaly, over all points in the region $180^{\circ} \mathrm{E}$ to $120^{\circ} \mathrm{E}, 40^{\circ} \mathrm{N}$ to $60^{\circ} \mathrm{N}$ having a negative SLP anomaly during El Niño. 
trends and variations over many parts of the world. For example, since IPCC (2007a) more than 2000 scientific articles have been published, which include $\mathrm{NAO}, \mathrm{AO}$, or NAM in either the title or abstract. This assessment focusses on recent research on these modes that is most relevant for future regional climate change. Past behaviour of these modes inferred from observations is assessed in Section 2.7.8.

\subsubsection{Northern Modes}

The NAO is a well-established dipolar mode of climate variability having opposite variations in sea level pressure between the Atlantic subtropical high and the Iceland/Arctic low (Wanner et al., 2001; Hurrell et al., 2003; Budikova, 2009). It is strongly associated with the tropospheric jet, storms (see Section 14.6.2), and blocking that determine the weather and climate over the North Atlantic and surrounding continents (Hurrell and Deser, 2009; Box 14.2). The NAO exists in boreal summer as well as in boreal winter, albeit with different physical characteristics (Sun et al., 2008; Folland et al., 2009).

Over the North Pacific, there is a similar wintertime dipolar mode known as the NPO associated with north-south displacements of the Asian-Pacific jet stream and the Pacific storm track. The NPO influences winter air temperature and precipitation over much of western North America as well as sea ice over the Pacific sector of the Arctic, more so than either ENSO (Section 14.4) or the PNA (Linkin and Nigam, 2008).

These dipolar modes have been interpreted as the regional manifestation of an annular mode in sea level pressure known as the Arctic Oscillation (AO; Thompson and Wallace, 1998) or the Northern Annular Mode (NAM; Thompson and Wallace, 2000). The AO (NAM at $1000 \mathrm{hPa)}$ index and the NAO index (see Box 2.5, Table 1) are strongly correlated but the $A O$ spatial pattern is more zonally symmetric and so differs from the NAO over the N. Pacific (Ambaum et al., 2001; Feldstein and Franzke, 2006). Hereafter, the term NAO is used to denote NAO, AO and NAM in boreal winter unless further distinction is required.

Climate models are generally able to simulate the gross features of NAO and NPO (see Section 9.5.3.2). It has been argued that these modes may be a preferred pattern of response to climate change (Gerber et al., 2008). However, this is not supported by a detailed examination of the vertical structure of the simulated global warming response (Woollings, 2008). Hori et al. (2007) noted that NAO variability did not change substantially in the Special Report on Emission Scenarios (SRES)-A1B and 20th century scenarios and so concluded that the trend in the NAO index (defined relative to a historical mean state) is a result of an anthropogenic trend in the basic mean state rather than due to changes in NAO variability. However, other research indicates that there is a coherent two-way interaction between the trend in the mean state and the NAO-like modes of variability - the mode and/or regime structure change due to changes in the mean state (Branstator and Selten, 2009 ; Barnes and Polvani, 2013). Section 14.6.2 assesses the jet and storm track changes associated with the projected responses.

Model simulations have underestimated the magnitude of the large positive trend from 1960-2000 in winter NAO observations, which now appears to be more likely due to natural variability rather than anthropogenic influences (see Section 10.3.3.2). Some studies have even considered NAO to be a source of natural variability that needs to be removed before detection and attribution of anthropogenic changes (Zhang et al., 2006). Detection of regional surface air temperature response to anthropogenic forcing has been found to be robust to the exclusion of model-simulated AO and PNA changes (Wu and Karoly, 2007). Model projections of wintertime European precipitation have been shown to become more consistent with observed trends after removal of trends due to NAO (Bhend and von Storch, 2008). Underestimation of trends in NAO can lead to biases in projections of regional climate, for example, Arctic sea ice (Koldunov et al., 2010).

Underestimation of NAO long-term variability may be due to missing or poorly represented processes in climate models. Recent observational and modelling studies have helped to confirm that the lower stratosphere plays an important role in explaining recent more negative NAO winters and long-term trends in NAO (Scaife et al., 2005; Dong et al., 2011; Ouzeau et al., 2011; Schimanke et al., 2011). This is supported by evidence that seasonal forecasts of NAO can be improved by inclusion of the stratospheric Quasi-Biennial Oscillation (QBO; Boer and Hamilton, 2008; Marshall and Scaife, 2010). Other studies have found that observed changes in stratospheric water vapour changes from 1965-1995 led to an impact on NAO simulated by a model, and have suggested that changes in stratospheric water vapour may be another possible pathway for communicating tropical forcing to the extratropics (Joshi et al., 2006; Bell et al., 2009). There is growing evidence that future NAO projections are sensitive to how climate models resolve stratospheric processes and troposphere-stratosphere interactions (Sigmond and Scinocca, 2010; Scaife et al., 2011a; Karpechko and Manzini, 2012).

Several recent studies of historical data have found a positive association between solar activity and NAO (Haigh and Roscoe, 2006; Kodera et al., 2008; Lockwood et al., 2010), while other studies have found little imprint of solar and volcanic forcing on NAO (Casty et al., 2007). Positive associations between NAO and solar forcing have been reproduced in recent modelling studies (Lee et al., 2008; Ineson et al., 2011) but no significant changes were found in CMIP5 projections of NAO due to changes in solar irradiance or aerosol forcing.

Observational studies have noted weakening of NAO during periods of reduced Arctic sea ice (Strong et al., 2009; Wu and Zhang, 2010). Several modelling studies have also shown a negative NAO response to the partial removal of sea ice in the Arctic or high latitudes (Kvamsto et al., 2004; Magnusdottir et al., 2004; Seierstad and Bader, 2009; Deser et al., 2010c; Screen et al., 2012). However, the strength and timing of the response to sea ice loss varies considerably between studies, and can be hard to separate from common responses to warming of the troposphere and from natural climate variability. The impact of sea ice loss in individual years on NAO is small and hard to detect (Bluthgen et al., 2012). Reviews of the emerging literature on this topic can be found in Budikova (2009) and Bader et al. (2011).

The NPO contributes to the excitation of ENSO events via the 'Seasonal Footprinting Mechanism' (SFM; Anderson, 2003; Vimont et al., 2009; Alexander et al., 2010). Some studies indicate that warm events in the central tropical Pacific Ocean may in turn excite the NPO (Di Lorenzo et al., 2009). 
Recent multi-model studies of NAO (Hori et al., 2007; Karpechko, 2010; Zhu and Wang, 2010; Gillett and Fyfe, 2013) reconfirm the small positive response of boreal winter NAO indices to GHG forcing noted in earlier studies reported in AR4 (Kuzmina et al., 2005; Miller et al., 2006; Stephenson et al., 2006). Projected trends in wintertime NAO indices are generally found to have small amplitude compared to natural internal variations (Deser et al., 2012). Furthermore, there is substantial variation in NAO projections from different climate models. For example, one study found no significant NAO trends in two simulations with the ECHAM4/OPYC3 model (Fischer-Bruns et al., 2009), whereas another study found a strong positive trend in NAO in the ECHAM5/MPI-OM SRES A1B simulations (Müller and Roeckner, 2008). The model dependence of the response is an important source of uncertainty in the regional climate change response (Karpechko, 2010). A multi-model study of 24 climate model projections suggests that there are no major changes in the NPO due to greenhouse warming (Furtado et al., 2011).

Figures $14.16 \mathrm{a}, \mathrm{b}$ summarize the wintertime NAO and NAM indices simulated by models participating in the CMIP5 experiment (Gillett and Fyfe, 2013). The multi-model mean of the NAO and NAM indices are similar and exhibit small linear trends in agreement with those shown for the NAM index in AR4 (AR4, Figure 10.17a). The multi-model mean projected increase of around 1 to $2 \mathrm{hPa}$ from 1850 to 2100 is smaller than the spread of around 2 to $4 \mathrm{hPa}$ between model simulations (Figure 14.16).
Some differences in model projections can be accounted for by changes in the NAO spatial pattern, for example, northeastward shifts in NAO centres of action have been found to be important for estimating the trend in the NAO index (Ulbrich and Christoph, 1999; Hu and Wu, 2004). Individual model simulations have shown the spatial extent of NAO influence decreases with GHG forcing (Fischer-Bruns et al., 2009), a positive feedback between jet and storm tracks that enhances a poleward shift in the NAO pattern (Choi et al., 2010), and changes in the NAO pattern but with no changes in the propagation conditions for Rossby waves (Brandefelt, 2006). One modelling study found a trend in the correlation between NAO and ENSO during the 21st century (Muller and Roeckner, 2006). Such changes in the structure of NAO and/or its interaction with other modes of variability would could lead to important regional climate impacts.

\subsubsection{Southern Annular Mode}

The Southern Annular Mode (SAM, also known as Antarctic Oscillation, AAO), is the leading mode of climate variability in the SH extratropics, describing fluctuations in the latitudinal position and strength of the mid-latitude eddy-driven westerly jet (see Box 2.5; Section 9.5.3.2). SAM variability has a major influence on the climate of Antarctica, Australasia, southern South America and South Africa (Watterson, 2009; Thompson et al., 2011 and references therein). (a) NAO
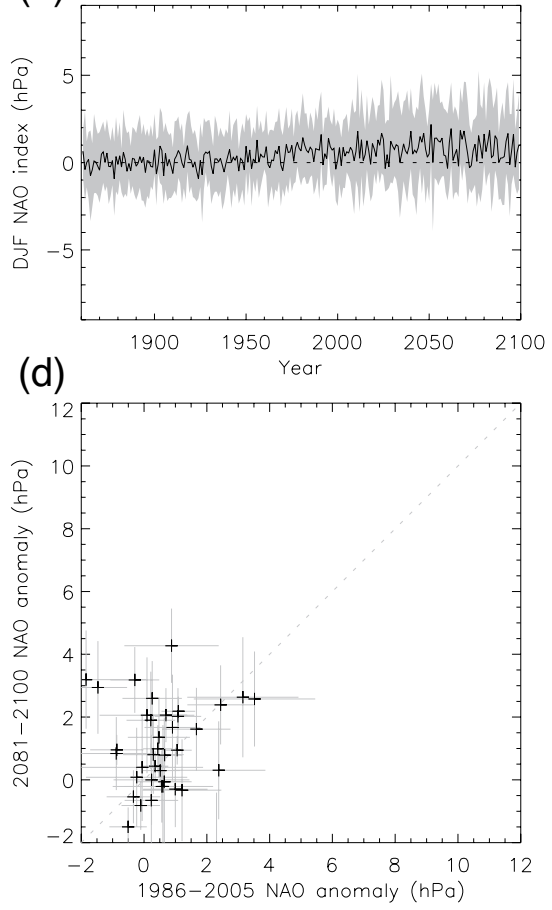

(b) NAM

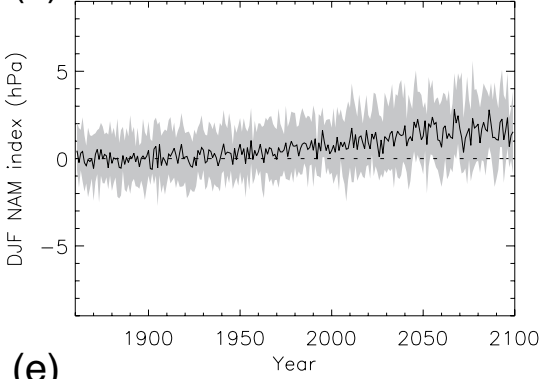

(e)

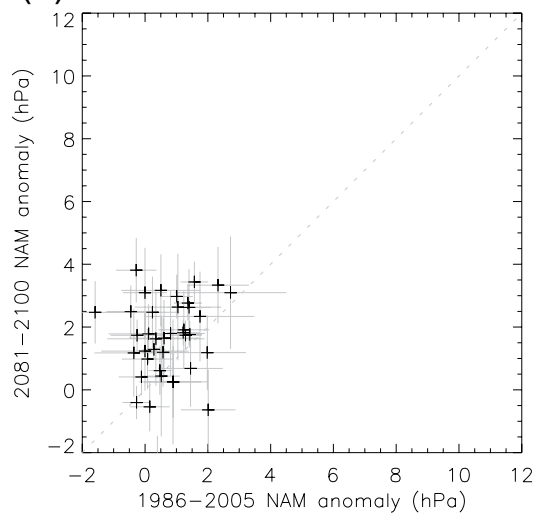

(c) SAM
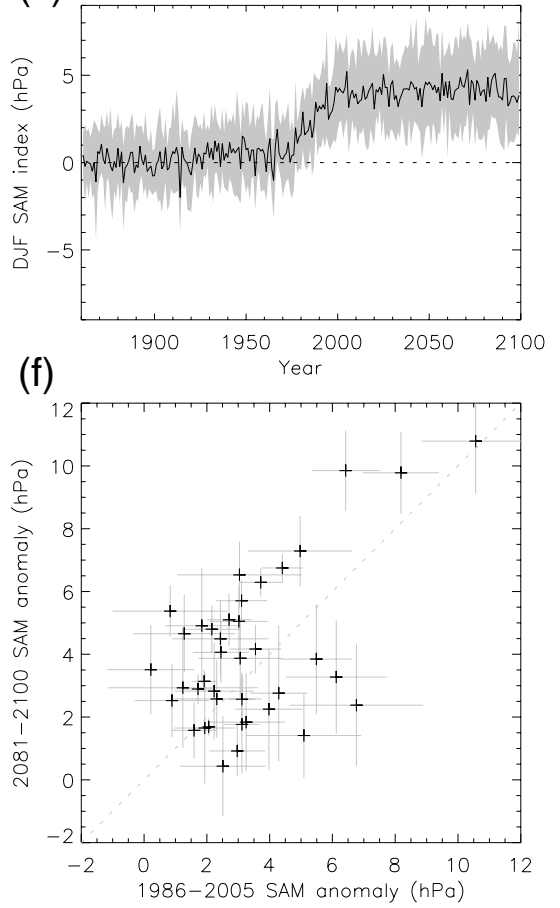

Figure 14.16 | Summary of multi-model ensemble simulations of wintertime (December to February) mean North Atlantic Oscillation (NAO), Northern Annular Mode (NAM) and Southern Annular Mode (SAM) sea level pressure (SLP) indices for historical and RCP4.5 scenarios produced by 39 climate models participating in CMIP5. Panels (a)-(c) show time series of the ensemble mean (black line) and inter-quartile range (grey shading) of the mean index for each model. Panels (d)-(f) show scatter plots of individual model $2081-2100$ time means versus 1986-2005 time means (black crosses) together with $(-2,+2)$ standard error bars. The NAO index is defined here as the difference of regional averages: (90 $\mathrm{W}$ to $60^{\circ} \mathrm{E}, 20^{\circ} \mathrm{N}$ to $55^{\circ} \mathrm{N}$ ) minus $\left(90^{\circ} \mathrm{W}\right.$ to $60^{\circ} \mathrm{E}, 55^{\circ} \mathrm{N}$ to $90^{\circ} \mathrm{N}$ ) (see Stephenson et al., 2006). The NAM and SAM are defined as zonal indices: NAM as the difference in zonal mean SLP at $35^{\circ} \mathrm{N}$ and $65^{\circ} \mathrm{N}$ (Li and Wang, 2003) and SAM as the difference in zonal mean SLP at $40^{\circ} \mathrm{S}$ and $65^{\circ} \mathrm{S}$ (Gong and Wang, 1999). All indices have been centred to have zero time mean from 1861-1900. Comparison of simulated and observed trends from 1961-2011 is shown in Figure 10.13. 
The physical mechanisms of the SAM are well understood, and the SAM is well represented in climate models, although the detailed spatial and temporal characteristics vary between models (Raphael and Holland, 2006). In the past few decades the SAM index has exhibited a positive trend in austral summer and autumn (Figure 14.16, Marshall, 2007; Jones et al., 2009b), a change attributed to the effects of ozone depletion and, to a lesser extent, the increase in GHGs (Thompson et al., 2011, see also Section 10.3.3.3). It is likely that these two factors will continue to be the principal drivers into the future, but as the ozone hole recovers they will be competing to push the SAM in opposite directions (Arblaster et al., 2011; Thompson et al., 2011; Bracegirdle et al., 2013), at least during late austral spring and summer, when ozone depletion has had its greatest impact on the SAM. The SAM is also influenced by teleconnections to the tropics, primarily associated with ENSO (Carvalho et al., 2005; L'Heureux and Thompson, 2006). Changes to the tropical circulation, and to such teleconnections, as the climate warms could further affect SAM variability (Karpechko et al., 2010). See Supplementary Material Section 14.SM.3.1 for further details on the observed variability of SAM.

The CMIP3 models projected a continuing positive trend in the SAM in both summer and winter (Miller et al., 2006). However, those models generally had poor simulations of stratospheric ozone, and tended to underestimate natural variability and to misrepresent observed trends in the SAM, indicating that care should be taken in interpretation of their future SAM projections (Fogt et al., 2009). Arblaster et al. (2011) showed that there can be large differences in the sensitivity of these models to $\mathrm{CO}_{2}$ increases, which affects their projected trends in the SAM.

Since the AR4 a number of chemistry-climate models (CCMs) have been run that have fully interactive stratospheric chemistry, although unlike coupled atmosphere ocean models they are usually not coupled to the oceans (see also Sections 9.1.3.2.8 and 9.4.6.2). The majority of CCMs and coupled models, which generally compare well to reanalyses (Gerber et al., 2010) although many exhibit biases in their placement of the SH eddy-driven jet (Wilcox et al., 2012; Bracegirdle et al., 2013), indicate that through to at least the mid-21st century the current observed SAM changes are neutralized or reversed during austral summer (Perlwitz et al., 2008; Son et al., 2010; Polvani et al., 2011; Bracegirdle et al., 2013). Figure 14.16 shows the projected ensemble-mean future SAM index evolution during DJF from a suite of CMIP5 models, suggesting that the recent positive trend will weaken considerably as stratospheric ozone concentrations recover over southern high latitudes.

Projected 21st century changes in the SAM, and the closely associated SH eddy-driven jet position, vary by season (Gillett and Fyfe, 2013), and are sensitive to the rate of ozone recovery (Son et al., 2010; Eyring et al., 2013) and to GHG emissions scenario (Swart and Fyfe, 2012; Eyring et al., 2013). In the RCP2.6 scenario, with small increases in GHGs, ozone recovery may dominate in austral summer giving a small projected equatorward jet shift (Eyring et al., 2013) with little change in the annual mean jet position (Swart and Fyfe, 2012). In RCP8.5 large GHG increases are expected to dominate, giving an ongoing poleward shift of the SH jet in all seasons (Swart and Fyfe, 2012; Eyring et al., 2013). In RCP4.5 the influences of ozone recovery and GHG increases are expected to approximately balance in austral summer, with an ongoing poleward jet shift projected in the other seasons (Swart and Fyfe, 2012; Eyring et al., 2013; Gillett and Fyfe, 2013).

\subsubsection{Assessment Summary}

Future boreal wintertime NAO is very likely to exhibit large natural variations and trend of similar magnitude to that observed in the past; is very likely to be differ quantitatively from individual climate model projections; is likely to become slightly more positive (on average) due to increases in GHGs. The austral summer/autumn positive trend in SAM is likely to weaken considerably as ozone depletion recovers through to the mid-21st century. There is medium confidence from recent studies that projected changes in NAO and SAM are sensitive to boundary processes, which are not yet well represented in many climate models currently used for projections, for example, stratosphere-troposphere interaction, ozone chemistry, solar forcing and atmospheric response to Arctic sea ice loss. There is low confidence in projections of other modes such as the NPO due to the small number of modelling studies.

\section{Box 14.2 | Blocking}

Atmospheric blocking is associated with persistent, slow-moving high-pressure systems that interrupt the prevailing westerly winds of middle and high latitudes and the normal eastward progress of extratropical storm systems. Overall, blocking activity is more frequent at the exit zones of the jet stream and shows appreciable seasonal variability in both hemispheres, reaching a maximum in winterspring and a minimum in summer-autumn (e.g., Wiedenmann et al., 2002). In the Northern Hemisphere (NH), the preferred locations for winter blocking are the North Atlantic and North Pacific, whereas continental blocks are relatively more frequent in summer (Tyrlis and Hoskins, 2008; Barriopedro et al., 2010). Southern Hemisphere (SH) blocking is less frequent than in the NH, and it tends to be concentrated over the Southeast Pacific and the Indian Ocean (Berrisford et al., 2007).

Blocking is a complex phenomenon that involves large- and small-scale components of the atmospheric circulation, and their mutual interactions. Although there is not a widely accepted blocking theory, transient eddy activity is considered to play an important role in blocking occurrence and maintenance through feedbacks between the large-scale flow and synoptic eddies (e.g., Yamazaki and Itoh, 2009). (continued on next page) 
Blocking is an important component of intraseasonal variability in the extratropics and causes climate anomalies over large areas of Europe (Trigo et al., 2004; Masato et al., 2012), North America (Carrera et al., 2004), East Asia (e.g., Wang et al., 2010; Cheung et al., 2012), high-latitude regions of the SH (Mendes et al., 2008) and Antarctica (Massom et al., 2004; Scarchilli et al., 2011). Blocking can also be responsible for extreme events (e.g., Buehler et al., 2011; Pfahl and Wernli, 2012), such as cold spells in winter (e.g., 2008 in China, Zhou et al., 2009d; or 2010 in Europe, Cattiaux, 2010) and summer heat waves in the NH (e.g., 2010 in Russia, Matsueda, 2011; Lupo et al., 2012) and in southern Australia (Pezza et al., 2008).

At interannual time scales, there are statistically significant relationships between blocking activity and several dominant modes of atmospheric variability, such as the NAO (Section 14.5.1) and wintertime blocking in the Euro-Atlantic sector (Croci-Maspoli et al., 2007a; Luo et al., 2010), the winter PNA (Section 14.7.1) and blocking frequency in the North Pacific (Croci-Maspoli et al., 2007a), or the SAM (Section 14.5.2) and winter blocking activity near the New Zealand sector (Berrisford et al., 2007). Multi-decadal variability in winter blocking over the North Atlantic and the North Pacific seem to be related, respectively, with the Atlantic Meridional Overturning Circulation (AMOC; Häkkinen et al., 2011; Section 14.7.6) and the Pacific Decadal Oscillation (PDO; Chen and Yoon, 2002; Section 14.7.3), although this remains an open question.

Other important scientific issues related to the blocking phenomenon include the mechanisms of blocking onset and maintenance, twoway interactions between blocking and stratospheric processes (e.g., Martius et al., 2009; Woollings et al., 2010), influence on blocking of slowly varying components of the climate system (sea surface temperature (SST), sea ice, etc., Liu et al., 2012b), and external forcings.

The most consistent long-term observed trends in blocking for the second half of the 20th century are the reduced winter activity over the North Atlantic (e.g., Croci-Maspoli et al., 2007b), which is consistent with the observed increasing North Atlantic Oscillation (NAO) trend from the 1960s to the mid-1990s (Section 2.7.8), as well as an eastward shift of intense winter blocking over the Atlantic and Pacific Oceans (Davini et al., 2012). The apparent decreasing trend in SH blocking activity (e.g., Dong et al., 2008) seems to be in agreement with the upward trend in the SAM.

The AR4 (Section 8.4.5) reported a tendency for General Circulation Models (GCMs) to underestimate NH blocking frequency and persistence, although most models were able to capture the preferred locations for blocking occurrence and their seasonal distributions. Several intercomparison studies based on a set of CMIP3 models (Scaife et al., 2010; Vial and Osborn, 2012) revealed some progress in the simulation of NH blocking activity, mainly in the North Pacific, but only modest improvements in the North Atlantic. In the SH, blocking frequency and duration was also underestimated, particularly over the Australia-New Zealand sector (Matsueda et al., 2010). CMIP5 models still show a general blocking frequency underestimation over the Euro-Atlantic sector, and some tendency to overestimate North Pacific blocking (Section 9.5.2.2), with considerable inter-model spread (Box 14.2, Figure 1).

Model biases in the mean flow, rather than in variability, can explain a large part of the blocking underestimation and they are usually evidenced as excessive zonality of the flow or systematic shifts in the latitude of the jet stream (Matsueda et al., 2010; Scaife et al., 2011 b; Barnes and Hartmann, 2012; Vial and Osborn, 2012; Anstey et al., 2013; Dunn-Sigouin and Son, 2013). Increasing the horizontal resolution in atmospheric GCMs with prescribed SSTs has been shown to significantly reduce blocking biases, particularly in the Euro-Atlantic sector and Australasian sectors (e.g., Matsueda et al., 2010; Jung et al., 2011; Dawson et al., 2012; Berckmans et al., 2013), while North Pacific blocking could be more sensitive to systematic errors in tropical SSTs (Hinton et al., 2009). Also blocking biases are smaller in those CMIP5 models with higher horizontal and vertical resolution (Anstey et al., 2013). However, the improvement of blocking simulation with increasing horizontal resolution is less clear in coupled models than in atmospheric GCMs with prescribed SSTs, indicating that both SSTs and the relative coarse resolution in OGCM (Scaife et al., 2011b) are important causes of blocking biases.

Most CMIP3 models projected significant reductions in NH annual blocking frequency (Barnes et al., 2012), particularly during winter, but CMIP5 models seem to indicate weaker decreases in the future (Dunn-Sigouin and Son, 2013) and a more complex response than that reported for CMIP3 models, including possible regional increases of blocking frequency in summer (Cattiaux et al., 2013; Masato et al., 2013). There is high agreement that winter blocking frequency over the North Atlantic and North Pacific will not increase under enhanced GHG concentrations (Barnes et al., 2012; Dunn-Sigouin and Son, 2013). Future strengthening of the zonal wind and meridional jet displacements may partially account for some of the projected changes in blocking frequency over the ocean basins of both hemispheres (Matsueda et al., 2010; Barnes and Hartmann, 2012; Dunn-Sigouin and Son, 2013). Future trends in blocking intensity and persistence are even more uncertain, with no clear signs of significant changes. How the location and frequency of blocking events will evolve in future are both critically important for understanding regional climate change in particular with respect to extreme conditions (e.g., Sillmann et al., 2011; de Vries et al., 2013). (continued on next page) 


\section{Box 14.2 (continued)}

In summary, the increased ability in simulating blocking in some models indicate that there is medium confidence that the frequency of $\mathrm{NH}$ and SH blocking will not increase, while trends in blocking intensity and persistence remain uncertain. The implications for blocking related regional changes in North America, Europe and Mediterranean and Central and North Asia are therefore also uncertain [Box 14.2 and $14.8 .3,14.8 .6,14.8 .8]$

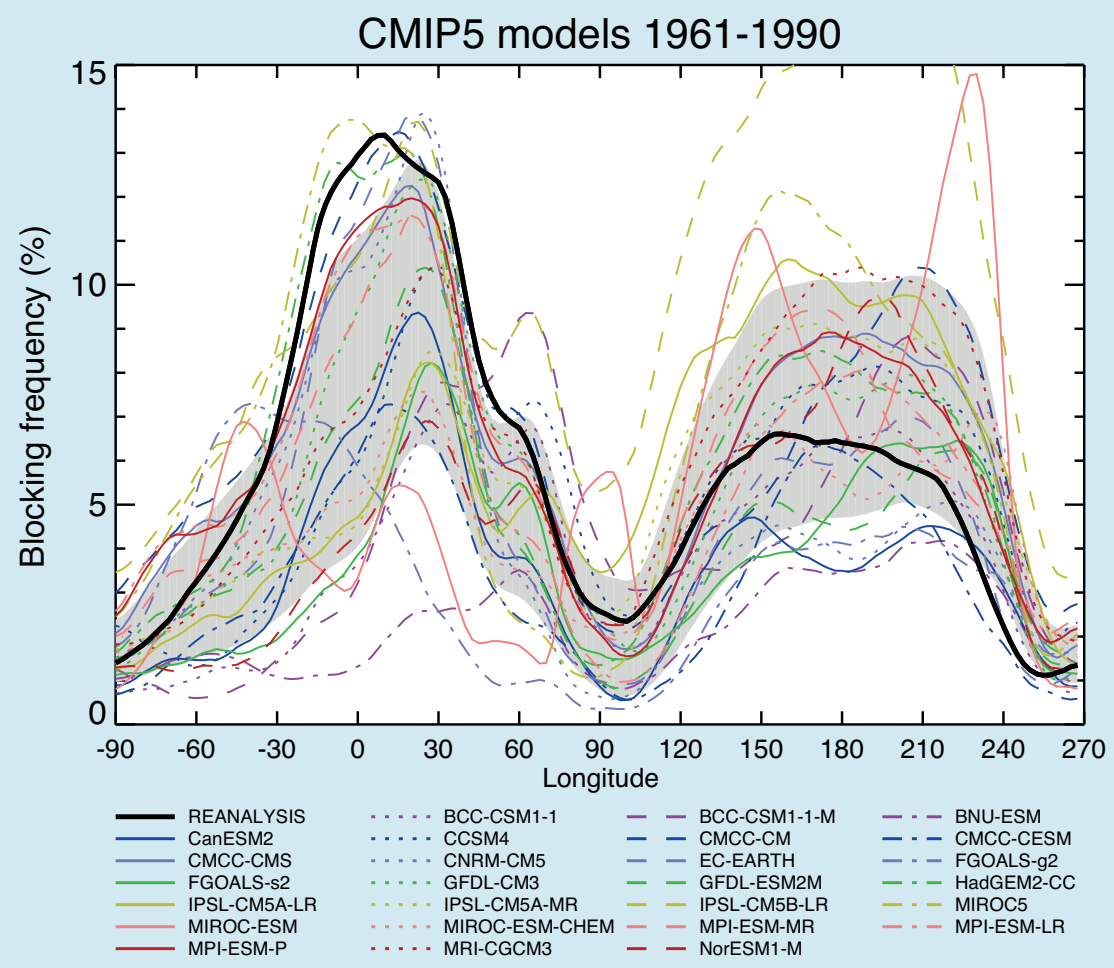

Box 14.2, Figure 1 | Annual mean blocking frequency in the NH (expressed in \% of time, that is, $1 \%$ means about 4 days per year) as simulated by a set of CMIP5 models (colour lines) for the 1961-1990 period of one run of the historical simulation. Grey shading shows the mean model result plus/minus one standard deviation. Black thick line indicates the observed blocking frequency derived from the National Centers for Environmental Prediction/National Center for Atmospheric Research (NCEP/NCAR) reanalysis. Only CMIP5 models with available $500 \mathrm{hPa}$ geopotential height daily data at http://pcmdi3.IInl.gov/esgcet/home.htm have been used. Blocking is defined as in Barriopedro et al. (2006), which uses a modified version of the(Tibaldi and Molteni, 1990) index. Daily data was interpolated to a common regular $2.5^{\circ} \times 2.5^{\circ}$ longitude-latitude grid before detecting blocking.

\subsection{Large-scale Storm Systems}

\subsubsection{Tropical Cyclones}

The potential for regional changes in future tropical cyclone frequency, track and intensity is of great interest, not just because of the associated negative effects, but also because tropical cyclones can play a major role in maintaining regional water resources (Jiang and Zipser, 2010; Lam et al., 2012; Prat and Nelson, 2012). Past and projected increases in human exposure to tropical cyclones in many regions (Peduzzi et al., 2012) heightens the interest further.

\subsubsection{Understanding the Causes of Past and Projected Regional Changes}

Detection of past trends in measures of tropical cyclone activity is constrained by the quality of historical records and uncertain quantification of natural variability in these measures (Knutson et al., 2010; Lee et al., 2012; Seneviratne et al., 2012). Observed regional climate variability generally represents a complex convolution of natural and anthropogenic factors, and the response of tropical cyclones to each factor is not yet well understood (see also Section 10.6.1.5 and Supplementary Material Section 14.SM.4.1.2). For example, the steady long-term increase in tropical Atlantic SST due to increasing GHGs can be dominated by shorter-term decadal variability forced by both external and internal factors (Mann and Emanuel, 2006; Baines and Folland, 2007; Evan et al., 2009, 2011a; Ting et al., 2009; Zhang and Delworth, 2009; Chang et al., 2011; Solomon and Newman, 2011; Booth et al., 2012; Camargo et al., 2012; Villarini and Vecchi, 2012). Similarly, tropical upper-tropospheric temperatures, which modulate tropical cyclone potential intensity (Emanuel, 2010), can be forced by slowly evolving changes in the stratospheric circulation of ozone (Brewer-Dobson circulation) due to climate change with occasional large amplitude and persistent changes forced by volcanic eruptions (Thompson and 
Solomon, 2009; Evan, 2012). This convolution of anthropogenic and natural factors, as represented in a climate model, has also been shown to be useful in prediction of Atlantic tropical storm frequency out to a few years (Smith et al., 2010).

In addition to greenhouse warming scenarios, tropical cyclones can also respond to anthropogenic forcing via different and possibly unexpected pathways. For example, increasing anthropogenic emissions of black carbon and other aerosols in South Asia has been linked to a reduction of SST gradients in the Northern Indian Ocean (Chung and Ramanathan, 2006; Meehl et al., 2008), which has in turn been linked to a weakening of the vertical wind shear in the region. Evan et al. (2011b) linked the reduced wind shear to the observed increase in the number of very intense storms in the Arabian Sea, including five very severe cyclones that have occurred since 1998, but the fundamental cause of this proposed linkage is not yet certain (Evan et al., 2012; Wang et al., 2012a). Furthermore, it is possible that a substantial part of the multi-decadal variability of North Atlantic SST is radiatively forced, via the cloud albedo effect, by what are essentially pollution aerosols emitted from North America and Europe (Baines and Folland, 2007; Booth et al., 2012), although the relative contribution of this forcing to the observed variability has been questioned (Zhang et al., 2013b). Note that in the North Atlantic, the evidence suggests that the reduction of pollution aerosols is linked to tropical SST increases, while in the northern Indian Ocean, increases in aerosol pollution have been linked to reduced vertical wind shear. Both of these effects (increasing SST and reduced shear) have been observed to be related to increased tropical cyclone activity.

Finally, in addition to interannual-to-multi-decadal forcing of tropical Atlantic SST via radiative dimming (Evan et al., 2009; Evan et al., 2011a), dust aerosols have a large and more immediate in situ effect on the regional thermodynamic and kinematic environment (Dunion and Marron, 2008; Dunion, 2011), and Saharan dust storms-whose frequency has been linked to atmospheric $\mathrm{CO}_{2}$ concentration (Mahowald, 2007) - have also been linked to reduced strengthening of tropical cyclones (Dunion and Velden, 2004; Wu, 2007). Direct in situ relationships have also been identified between aerosol pollution concentrations and tropical cyclone structure and intensity (Khain et al., 2008, 2010; Rosenfeld et al., 2011). Thus, when assessing changes in tropical cyclone activity, it is clear that detection and attribution aimed simply at long-term linear trends forced by increasing well-mixed GHGs is not adequate to provide a complete picture of the potential anthropogenic contributions to the changes in tropical cyclone activity that have been observed (Section 10.6).

\subsubsection{Regional Numerical Projections}

Similar to observational analyses, confidence in numerical simulations of tropical cyclone activity (Supplementary Material Tables 14.SM.1 to 14.SM.4) is reduced when model spatial domain is reduced from global to region-specific (IPCC SREX Box 3.2; see also Section 9.5.4.3). The assessment provided by Knutson et al. (2010) of projections based on the SRES A1B scenario concluded that it is likely that the global frequency of tropical cyclones will either decrease or remain essentially unchanged while mean intensity (as measured by maximum wind speed) increases by +2 to $+11 \%$ and tropical cyclone rainfall rates increase by about $20 \%$ within $100 \mathrm{~km}$ of the cyclone centre. However, inter-model differences in regional projections lead to lower confidence in basin-specific projections, and confidence is particularly low for projections of frequency within individual basins. For example, a recent study by Ying et al. (2012) showed that numerical projections of 21st century changes in tropical cyclone frequency in the western North Pacific range broadly from $-70 \%$ to $+60 \%$, while there is better model agreement in measures of mean intensity and precipitation, which are projected to change in the region by $-3 \%$ to $+18 \%$ and $+5 \%$ to $+30 \%$, respectively. The available modelling studies that are capable of producing very strong cyclones typically project substantial increases in the frequency of the most intense cyclones and it is more likely than not that this increase will be larger than $10 \%$ in some basins (Emanuel et al., 2008; Bender et al., 2010; Knutson et al., 2010, 2013; Yamada et al., 2010; Murakami et al., 2012). It should be emphasized that this metric is generally more important to physical and societal impacts than overall frequency or mean intensity.

As seen in Tables 14.SM.1 to 14.SM.4 of the Supplementary Material, as well as the previous assessments noted above, model projections often vary in the details of the models and the experiments performed, and it is difficult to objectively assess their combined results to form a consensus, particularly by region. It is useful to do this after normalizing the model output using a combination of objective and subjective expert judgements. The results of this are shown in Figure 14.17, and are based on a subjective normalization of the model output to four common metrics under a common future scenario projected through the 21 st century. The global assessment is essentially the same as Knutson et al. (2010) and the assessment of projections in the western North Pacific is essentially unchanged from Ying et al. (2012). The annual frequency of tropical cyclones is generally projected to decrease or remain essentially unchanged in the next century in most regions although as noted above, the confidence in the projections is lower in specified regions than global projections. The decrease in storm frequency is apparently related to a projected decrease of upward deep convective mass flux and increase in the saturation deficit of the middle troposphere in the tropics associated with global warming (Bengtsson et al., 2007; Emanuel et al., 2008, 2012; Zhao et al., 2009; Held and Zhao, 2011; Murakami et al., 2012; Sugi et al., 2012; Sugi and Yoshimura, 2012).

A number of experiments that are able to simulate intense tropical cyclones project increases in the frequency of these storms in some regions, although there are presently only limited studies to assess and there is insufficient data to draw from in most regions to make a confident assessment (Figure 14.17). Confidence is somewhat better in the North Atlantic and western North Pacific basins where an increase in the frequency of the strongest storms is more likely than not. The models generally project an increase in mean lifetime-maximum intensity of simulated storms (Supplementary Material Table 14.SM.3), which is consistent with a projected increase in the frequency in the more intense storms (Elsner et al., 2008). The projected increase in intensity concurrent with a projected decrease in frequency can be argued to result from a difference in scaling between projected changes in surface enthalpy fluxes and the Clausius-Clapeyron relationship associated with the moist static energy of the middle troposphere (Emanuel et al., 2008). The increase in rainfall rates associated with 
tropical cyclones is a consistent feature of the numerical models under greenhouse warming as atmospheric moisture content in the tropics and tropical cyclone moisture convergence is projected to increase (Trenberth et al., 2005, 2007a; Allan and Soden, 2008; Knutson et al., $2010,2013)$. Although no broad-scale, detectable long-term changes in tropical cyclone rainfall rates have been reported, preliminary evidence for a detectable anthropogenic increase in atmospheric moisture content over ocean regions has been reported by Santer et al. (2007).

A number of studies since the AR4 have attempted to project future changes in tropical cyclone tracks and genesis at inter- or intra-basin scale (Leslie et al., 2007; Vecchi and Soden, 2007b; Emanuel et al., 2008; Yokoi and Takayabu, 2009; Zhao et al., 2009; Li et al., 2010b; Murakami and Wang, 2010; Lavender and Walsh, 2011; Murakami et al., 2011a, 2013). These studies suggest that projected changes in tropical cyclone activity are strongly correlated with projected changes in the spatial pattern of tropical SST (Sugi et al., 2009; Chauvin and Royer, 2010; Murakami et al., 2011b; Zhao and Held, 2012) and associated weakening of the Pacific Walker Circulation (Vecchi and Soden, 2007a), indicating that reliable projections of regional tropical cyclone activity depend critically on the reliability of the projected pattern of SST changes. However, assessing changes in regional tropical cyclone frequency is still limited because confidence in projections critically depend on the performance of control simulations (Murakami and Sugi, 2010), and current climate models still fail to simulate observed temporal and spatial variations in tropical cyclone frequency (Walsh et al., 2012). As noted above, tropical cyclone genesis and track variability is modulated in most regions by known modes of atmosphere-ocean variability. The details of the relationships vary by region (e.g., El Niño events tend to cause track shifts in western North Pacific typhoons and tend to suppress Atlantic storm genesis and development). Similarly, it has been demonstrated that accurate modelling of tropical cyclone activity fundamentally depends on the model's ability to reproduce these modes of variability (e.g., Yokoi and Takayabu, 2009). Reliable projections of future tropical cyclone activity, both global and regional, will then depend critically on reliable projections of the behaviour of these modes of variability (e.g., ENSO) under global warming, as well as an adequate understanding of their physical links with tropical cyclones. At present there is still uncertainty in their projected behaviours (e.g., Section 14.4).

The reduction in signal-to-noise ratio that accompanies changing focus from global to regional scales also lengthens the emergence time scale (i.e., the time required for a trend signal to rise above the natural variability in a statistically significant way). Based on changes in tropical cyclone intensity predicted by idealized numerical simulations with carbon dioxide $\left(\mathrm{CO}_{2}\right)$-induced tropical SST warming, Knutson and Tuleya (2004) suggested that clearly detectable increases may not be manifest for decades to come. The more recent high-resolution dynamical downscaling study of Bender et al. (2010) supports this argument and suggests that the predicted increases in the frequency of the strongest Atlantic storms may not emerge as a statistically significant signal until the latter half of the 21 st century under the SRES A1B emissionscenario. However, regional forcing by agents other than GHGs,

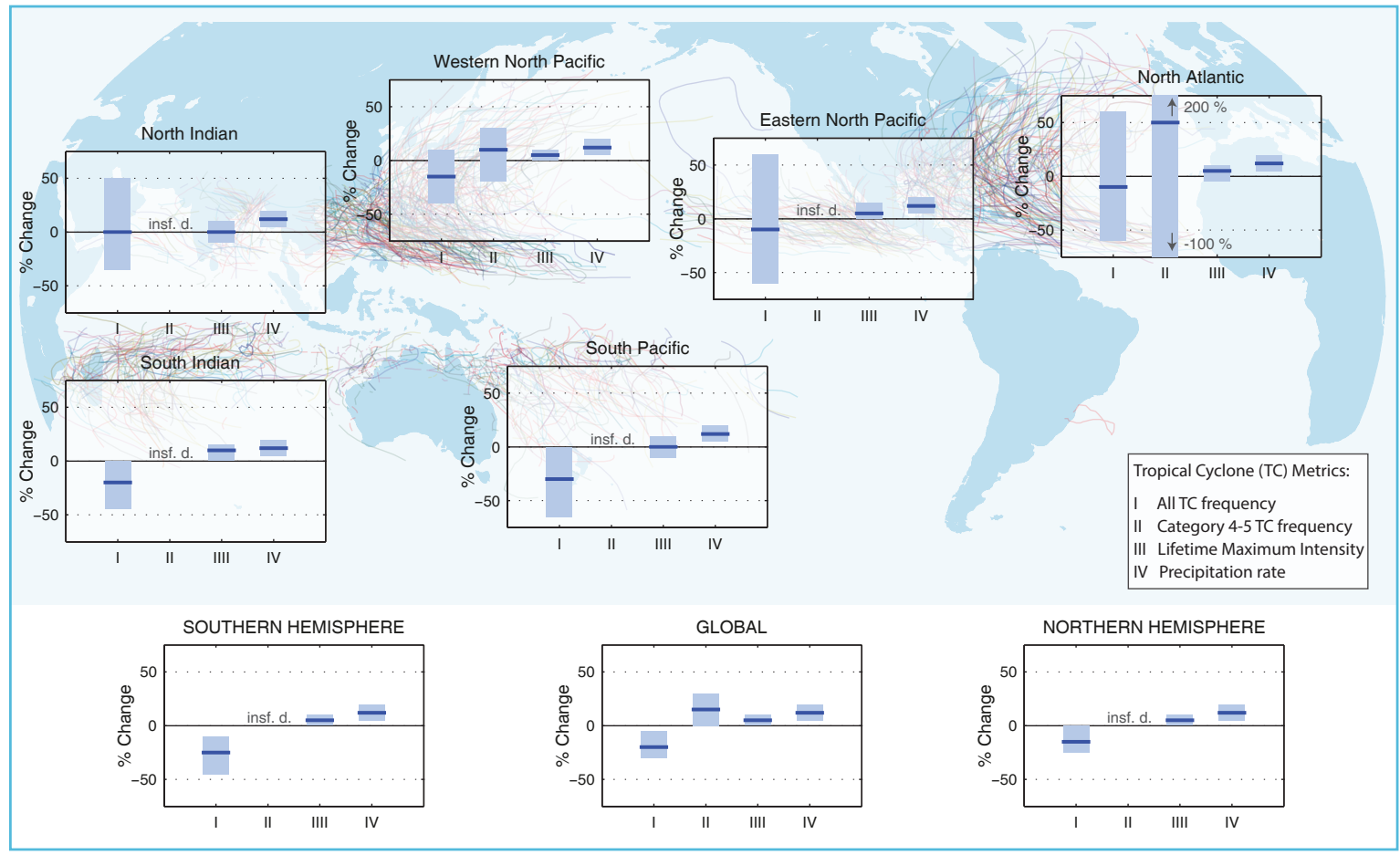

Figure 14.17| General consensus assessment of the numerical experiments described in Supplementary Material Tables 14.SM.1 to 14.SM.4. All values represent expected percent change in the average over period 2081-2100 relative to 2000-2019, under an A1B-like scenario, based on expert judgement after subjective normalization of the model projections. Four metrics were considered: the percent change in (I) the total annual frequency of tropical storms, (II) the annual frequency of Category 4 and 5 storms, (III) the mean Lifetime Maximum Intensity (LMI; the maximum intensity achieved during a storm's lifetime) and (IV) the precipitation rate within $200 \mathrm{~km}$ of storm centre at the time of LMI. For each metric plotted, the solid blue line is the best guess of the expected percent change, and the coloured bar provides the $67 \%$ (likely) confidence interval for this value (note that this interval ranges across $-100 \%$ to $+200 \%$ for the annual frequency of Category 4 and 5 storms in the North Atlantic). Where a metric is not plotted, there are insufficient data (denoted 'insf. d.') available to complete an assessment. A randomly drawn (and coloured) selection of historical storm tracks are underlain to identify regions of tropical cyclone activity. 
such as anthropogenic aerosols, is known to affect the regional climatic conditions differently (e.g., Zhang and Delworth, 2009), and there is evidence that tropical cyclone activity may have changed in some regions because of effects from anthropogenic aerosol pollution. The fidelity of the emergence time scales projected under A1B warming thus depends on the fidelity of $\mathrm{A} 1 \mathrm{~B}$ aerosol projections, which are known to be uncertain (Forster et al., 2007; Haerter et al., 2009).

\subsubsection{Extratropical Cyclones}

Some agreement on the response of storm tracks to anthropogenic forcing started to emerge in the climate model projections from CMIP3, with many models projecting a poleward shift of the storm tracks (Yin, 2005) and an expansion of the tropics (Lu et al., 2007). As stated in AR4 (Meehl et al., 2007) this response is particularly clear in the SH, but less clear in the NH. Although clearer in zonal mean responses (Yin, 2005), regional responses at different longitudes differ widely from this in many models (Ulbrich et al., 2008). There is a strong two-way coupling between storm tracks and the large-scale circulation (Lorenz and Hartmann, 2003; Robinson, 2006; Gerber and Vallis, 2007), which results in associated shifts in storm tracks and westerly jet streams (Raible, 2007; Athanasiadis et al., 2010).

\subsubsection{Process Understanding of Future Changes}

Future storm track change is the result of several different competing dynamical influences (Held, 1993; O'Gorman, 2010; Woollings, 2010). It is becoming apparent that the relatively modest storm track response in many models reflects the partial cancelling of opposing tendencies (Son and Lee, 2005; Lim and Simmonds, 2009; Butler et al., 2010).

One of the most important factors is the change in the meridional temperature gradient from which ETCs draw most of their energy. This gradient is projected to increase in the upper troposphere due to tropical amplification and decrease in the lower troposphere due to polar amplification, and it is still unclear whether this will lead to an overall increase or decrease in ETC activity. The projected response can involve an increase in eddy activity at upper levels and a decrease at lower levels (Hernandez-Deckers and von Storch, 2010), although in other models changes in low level eddy activity are more in line with the upper level wind changes (Mizuta et al., 2011; Wu et al., 2011; Mizuta, 2012). The projected warming pattern also changes vertical temperature gradients leading to increased stability at low latitudes and decreased stability at higher latitudes, and there is some modelling evidence that this may be a strong factor in the response (Lu et al., 2008, 2010; Kodama and Iwasaki, 2009; Lim and Simmonds, 2009). Increasing depth of the troposphere might also be important for future changes (Lorenz and DeWeaver, 2007).

Uncertainties in the projections of large-scale warming contribute to uncertainty in the storm track response (Rind, 2008). Several mechanisms have been proposed to explain how the storm tracks respond to the large-scale changes, including changes in eddy phase speed (Chen et al., 2007, 2008; Lu et al., 2008), eddy source regions (Lu et al., 2010) and eddy length scales (Kidston et al., 2011) with a subsequent effect on wave-breaking characteristics (Riviere, 2011). Furthermore, changes in the large-scale warming might also be partly due to changes in the storm tracks due to the two-way coupling between storm tracks and the large-scale flow. However, there is evidence that the amplitude of the tropical and polar warming are largely determined by atmospheric poleward heat fluxes set by local processes (Hwang and Frierson, 2010).

Local processes could prove important for the storm track response in certain regions, for example, sea ice loss (Kvamsto et al., 2004; Seierstad and Bader, 2009; Deser et al., 2010c; Bader et al., 2011) and spatial changes in SSTs (Graff and LaCasce, 2012). Local land-sea contrast in warming also has a local influence on baroclinicity along the eastern continental coastlines (Long et al., 2009; McDonald, 2011). It is not clear how the storm track responds to multiple forcings with some studies suggesting a linear response (Lim and Simmonds, 2009) while others suggest more complex interaction (Butler et al., 2010).

The projected increase in moisture content in a warmer atmosphere is also likely to have competing effects. Latent heating has been shown to play a role in invigorating individual ETCs, especially in the downstream development over eastern ocean (Dacre and Gray, 2009; Fink et al., 2009, 2012). However, there is evidence that the overall effect of moistening is to weaken ETCs by improving the efficiency of poleward heat transport and hence reducing the dry baroclinicity (Frierson et al., 2007; 0'Gorman and Schneider, 2008; Schneider et al., 2010; Lucarini and Ragone, 2011). Consistent with this, studies have shown that precipitation is projected to increase in ETCs despite no increase in wind speed intensity of ETCs (Bengtsson et al., 2009; Zappa et al., 2013b).

\subsubsection{Regional Projections}

Large-scale projections of ETCs are assessed in Section 12.4.4.3. This section complements this by presenting a more detailed assessment of regional changes.

Individual model projections of regional storm track changes are often comparable with the magnitude of interannual natural variability and so the changes are expected to be relevant for regional climate. However, the magnitude of the response is model dependent at any given location, especially over land (Harvey et al., 2012). There is also disagreement between different cyclone/storm track identification methods, even when applied to the same data (Raible et al., 2008; Ulbrich et al., 2009), although in the response to anthropogenic forcing, these differences appear mainly in the statistics of weak cyclones (Ulbrich et al., 2013). Conversely, when the same method is applied to different models the spread between the model responses is often larger than the ensemble mean response, especially in the NH (Ulbrich et al., 2008; Laine et al., 2009).

The poleward shift of the SH storm track remains one of the most reproducible projections, yet even here there is considerable quantitative uncertainty. This is partly associated with the varied model biases in jet latitude (Kidston and Gerber, 2010) although factors such as the varied cloud response may play a role (Trenberth and Fasullo, 2010). Many models project a similar poleward shift in the North Pacific (Bengtsson et al., 2006; Ulbrich et al., 2008; Catto et al., 2011), although this is often weaker compared to natural variability and often varies considerably between ensemble members (Pinto et al., 2007; McDonald, 2011). Poleward shifts are generally less clear at the surface than in the upper troposphere (Yin, 2005; McDonald, 2011; Chang et 
al., 2012), which reduces their relevance for regional impacts. However, a shift in extreme surface winds is still detectable in the zonal mean, especially in the subtropics and the southern high latitudes (Gastineau and Soden, 2009). A weakening of the Mediterranean storm track is a particularly robust response (Pinto et al., 2007; Loeptien et al., 2008; Ulbrich et al., 2009; Donat et al., 2011) for which increasing static stability is important (Raible et al., 2010). In general, the storm track response in summer is weaker than in winter with less consistency between models (Lang and Waugh, 2011).

The response of the North Atlantic storm track is more complex than a poleward shift in many models, with an increase in storm activity and a downstream extension of the storm track into Europe (Bengtsson et al., 2006; Pinto et al., 2007; Ulbrich et al., 2008; Catto et al., 2011; McDonald, 2011). In some models this regional response is important (Ulbrich et al., 2009), with storm activity over Western Europe increasing by $50 \%$ (McDonald, 2011) or by an amount comparable to the natural variability (Pinto et al., 2007; Woollings et al., 2012). The return periods of intense cyclones are shortened (Della-Marta and Pinto, 2009) with impact on potential wind damage (Leckebusch et al., 2007; Donat et al., 2011) and economic losses (Pinto et al., 2012). This response is related to the local minimum in warming in the North Atlantic ocean, which serves to increase the meridional temperature gradient on its southern side (Laine et al., 2009; Catto et al., 2011). The minimum in warming arises due to the weakening of northward ocean heat transports by the Atlantic Meridional Overturning Circulation (AMOC), and the varying $A M O C$ responses of the models can account for a large fraction of the variance in the Atlantic storm track projections (Woollings et al., 2012). CMIP5 models show a similar, albeit weaker extension of the storm track towards Europe, flanked by reductions in cyclone activity on both the northern and southern sides (Harvey et al., 2012; Zappa et al., 2013b). Despite large biases in the mean state, the model responses were found to agree with one another within sampling variation caused by natural variability (Sansom et al., 2013). Colle et al. (2013) noted similar reductions but also found that the higher resolution CMIP5 models gave more realistic ETC performance in the historical period. The best 7 models were found to give projections of increased 10 to $20 \%$ increase in cyclone track density over the eastern USA, including 10 to $40 \%$ more intense ( $<980 \mathrm{hPa}$ ) cyclones.

There is general agreement that there will be a small global reduction in ETC numbers (Ulbrich et al., 2009). In individual regions there can be much larger changes which are comparable to natural variations, but these changes are not reproduced by the majority of the models (e.g., Donat et al., 2011). ETC intensities are particularly sensitive to the method and quantity used to define them, so there is little consensus on changes in intensity (Ulbrich et al., 2009). While there are indications that the absolute values of pressure minima deepen in future scenario simulations (Lambert and Fyfe, 2006), this is often associated with large-scale pressure changes rather than changes in the pressure gradients or winds associated with ETCs (Bengtsson et al., 2009; Ulbrich et al., 2009; McDonald, 2011). The CMIP5 model projections show little evidence of change in the intensity of winds associated with ETCs (Zappa et al., 2013b).

There are systematic storm track biases common to many models, which might have some influence on the projected storm track response to forcing (Chang et al., 2012). Some models with improved representation of the stratosphere have shown a markedly different circulation response in the $\mathrm{NH}$, with consequences for Atlantic/European storm activity in particular (Scaife et al., 2011a). Concerns over the skill of many models in representing both the stratosphere and the ocean mean that confidence in $\mathrm{NH}$ storm track projections remains low. Higher horizontal resolution can improve ETC representation, yet there are still relatively few high-resolution global models which have been used for storm track projections (Geng and Sugi, 2003; Bengtsson et al., 2009; Catto et al., 2011; Colle et al., 2013; Zappa et al., 2013a). Several studies have used RCMs to simulate storms at high resolution in particular regions. In multi-model experiments over Europe, the ETC response is more sensitive to the choice of driving GCM than the choice of RCM (Leckebusch et al., 2006; Donat et al., 2011), highlighting the importance of large-scale circulation uncertainties. There has been little work on potential changes to mesoscale storm systems, although it has been suggested that polar lows may reduce in frequency due to an increase in static stability (Zahn and von Storch, 2010). Higher resolution runs of one climate model also suggest an increase in intensity of autumn ETCs due to increased transitioning of Atlantic hurricanes (Haarsma et al., 2013).

\subsubsection{Assessment Summary}

The influence of past and future climate change on tropical cyclones is likely to vary by region, but the specific characteristics of the changes are not yet well understood, and the substantial influence of ENSO and other known climate modes on global and regional tropical cyclone activity emphasizes the need for more reliable assessments of future changes in the characteristics of these modes. Recent advances in understanding and phenomenological evidence for shorter-term effects on tropical cyclones from aerosol forcing are providing increasingly greater confidence that anthropogenic forcing has had a measurable effect on tropical cyclone activity in certain regions. Shorter term increases such as those observed in the Atlantic over the past 30 to 40 years appear to be robust and have been hypothesized to be related, in part, to regional external forcing by GHGs and aerosols, but the more steady century-scale trends that may be expected from $\mathrm{CO}_{2}$ forcing alone are much more difficult to assess given the data uncertainty in the available tropical cyclone records.

Although projections under 21 st century greenhouse warming indicate that it is likely that the global frequency of tropical cyclones will either decrease or remain essentially unchanged, concurrent with a likely increase in both global mean tropical cyclone maximum wind speed and rainfall rates, there is low confidence in region-specific projections of frequency and intensity. Still, based on high-resolution modelling studies, the frequency of the most intense storms, which are associated with particularly extensive physical effects, will more likely than not increase substantially in some basins under projected 21st century warming and there is medium confidence that tropical cyclone rainfall rates will increase in every affected region.

The global number of ETCs is unlikely to decrease by more than a few percent due to anthropogenic change. A small poleward shift is likely in the SH storm track, but the magnitude is model dependent. There is only medium confidence in projections of storm track shifts in the 
Northern Hemisphere. Nevertheless, model results suggests that it is more likely than not that the N. Pacific storm track will shift poleward, and that it is unlikely that the N. Atlantic storm track will respond with a simple poleward shift. There is low confidence in the magnitude of regional storm track changes, and the impact of such changes on regional surface climate.

\subsection{Additional Phenomena of Relevance}

\subsubsection{Pacific-South American Pattern}

The PSA pattern is a teleconnection from the tropics to SH extratropics through Rossby wave trains (Box 2.5). This pattern induces atmospheric circulation anomalies over South America, affecting extreme precipitation (Drumond and Ambrizzi, 2005; Cunningham and Cavalcanti, 2006; Muza et al., 2009; Vasconcellos and Cavalcanti, 2010). PSA trends for recent decades depend on the choice of indices and are hence uncertain (Section 2.7.8). The PSA pattern is reproduced in many model simulations (Solman and Le Treut, 2006; Vera and Silvestri, 2009; Bates, 2010; Rodrigues et al., 2011; Cavalcanti and Shimizu, 2012).

The intensification and westward displacement of the PSA wave pattern in projections of CMIP3 may be related to the increase in frequency and intensity of positive SST anomalies in the tropical Pacific by the end of the 21st century (2081-2100, Junquas et al., 2012). These perturbations of the PSA characteristics are linked with changes in SACZ dipole precipitation and affect South America precipitation (Section 14.3.1.3). The PSA pattern occurrence and implications for precipitation increase in the southeastern South America have been associated with the zonally asymmetric part of the global SST change in the equatorial Indian-western Pacific Oceans (Junquas et al., 2013).

Process understanding of the formation of the PSA gives medium confidence that future changes in the mean atmospheric circulation for austral summer will project on the PSA pattern thereby influencing the SACZ and precipitation over southeastern South America. However, the literature is not sufficient to assess more general changes in PSA, and confidence is low in its future projections.

\subsubsection{Pacific-North American Pattern}

The PNA pattern, as defined in Box 2.5, Table 1 and portrayed in Box 2.5, Figure 2 is a prominent mode of atmospheric variability over the North Pacific and the North American land mass. This phenomenon exerts notable influences on the temperature and precipitation variability in these regions (e.g., Nigam, 2003). The PNA pattern is related to ENSO events in the tropical Pacific (see Section 14.4), and also serves as a bridge linking ENSO and NAO variability (see Li and Lau, 2012). The data records indicate a significant positive trend in the wintertime PNA index over the past 60 years (see Table 2.14 and Box 2.5, Figure 1).

Stoner et al. (2009) have assessed the capability of 22 CMIP3 GCMs in replicating the essential aspects of the observed PNA pattern. Their results indicate that a majority of the models overestimate the fraction of variance explained by the PNA pattern, and that the spatial characteristics of PNA patterns simulated in 14 of the 22 models are in good agreement with the observations. The model-projected future evolution of the PNA pattern has not yet been fully assessed and therefore confidence in its future development is low.

\subsubsection{Pacific Decadal Oscillation/Inter-decadal Pacific Oscillation}

The Pacific Decadal Oscillation (PDO, Box 2.5) refers to the leading Empirical Orthogonal Function (EOF) of monthly SST anomalies over the North Pacific (north of $20^{\circ} \mathrm{N}$ ) from which the globally averaged SST anomalies have been subtracted (Mantua et al., 1997). It exhibits anomalies of one sign along the west coast of North America and of opposite sign in the western and central North Pacific (see also Section 9.5.3.6 and Chapter 11). The PDO is closely linked to fluctuations in the strength of the wintertime Aleutian Low Pressure System. The time scale of the PDO is around 20 to 30 years, with changes of sign between positive and negative polarities in the 1920s, the late 1940s, the late 1970 s and around 2000.

The extension of the PDO to the whole Pacific basin is known as the Inter-decadal Pacific Oscillation (IPO, Power et al., 1999). The IPO is nearly identical in form to the PDO in the NH but is defined globally, as a leading EOF (principal component) of 13-year lowpass-filtered global SST anomalies (Parker et al., 2007) and has substantial amplitude in the tropical and southern Pacific. The time series of the PDO and IPO correlate highly on an annual basis. The PDO/IPO pattern is considered to be the result of internal climate variability (Schneider and Cornuelle, 2005; Alexander, 2010) and has not been observed to exhibit a longterm trend. The PDO/IPO is associated with ENSO modulations, with more El Niño activity during the positive PDO/IPO and more La Niña activity during the negative PDO/IPO.

At the time of the AR4, little had been published on modelling of the PDO/IPO or of its evolution in future. In a recent study, Furtado et al. (2011) found that the PDO/IPO did not exhibit major changes in spatial or temporal characteristics under GHG warming in most of the 24 CMIP3 models used, although some models indicated a weak shift toward more occurrences of the negative phase of the PDO/IPO by the end of the 21st century (2081-2100, Lapp et al., 2012). However, given that the models strongly underestimate the PDO/IPO connection with tropical Indo-Pacific SST variations (Furtado et al., 2011; Lienert et al., 2011), the credibility of the projections remains uncertain. Furthermore, internal variability is so high that it is hard to detect any forced changes in the Aleutian Low for the next half a century (Deser et al., 2012; Oshima et al., 2012). Therefore confidence is low in projections of future changes in PDO/IPO.

\subsubsection{Tropospheric Biennial Oscillation}

The Tropospheric Biennial Oscillation (TBO; Meehl, 1997) is a proposed mechanism for the biennial tendency in large-scale drought and floods of south Asia and Australia. Multiple studies imply that TBO involves the Asian-Australian monsoon, the IOD and ENSO (Sections 14.2.2, 14.3.3 and 14.4; see also Supplementary Material Section 14.SM.5.2).

The IPO (Section 14.7.3) affects the decade-to-decade strength of the TBO. A major contributor to recent change in the TBO comes from 
increase of SST in the Indian Ocean that contributes to stronger trade winds in the Pacific, one of the processes previously identified with strengthening the TBO (Meehl and Arblaster, 2012). Thus, prediction of decadal variability assessed in Chapter 11 that can be associated, for example, with the IPO (e.g., Meehl et al., 2010) can influence the accuracy of shorter-term predictions of the TBO across the entire Indo-Pacific region (Turner et al., 2011), but the relevance for longer time scales is uncertain.

Since AR4, little work has been done to document the ability of climate models to simulate the TBO. However, Li et al. (2012b) showed that there is an overall improvement in the seasonality of monsoons rainfall related to changes in the TBO from CMIP3 to CMIP5, with most CMIP5 models better simulating both the monsoon timing and the very low rainfall rates outside of the monsoon season (see also Section 14.2.2). In addition they concluded that the India-Australia link seems to be robust in all models.

With regard to possible future behaviour of the TBO, no analysis using multiple GCMs has been made since the AR4. In models that more accurately simulate the TBO in the present-day climate, the TBO strengthens in a future warmer climate (Nanjundiah et al., 2005). However, as with ENSO (Section 14.4) and IOD (Section 14.3.3), internally generated decadal variability complicates the interpretation of such future changes. Therefore, it remains unclear how future changes in the TBO will emerge and how this may influence regional climate in the future. Confidence in the projected future changes in TBO remains low.

\subsubsection{Quasi-Biennial Oscillation}

The QBO is a near-periodic, large-amplitude, downward propagating oscillation in zonal winds in the equatorial stratosphere (Baldwin et al., 2001). It is driven by vertically propagating internal waves that are generated in the tropical troposphere (Plumb, 1977). The QBO has substantial effects on the global stratospheric circulation, in particular the strength of the northern stratospheric polar vortex as well as the extratropical troposphere (Boer, 2009; Marshall and Scaife, 2009; Garfinkel and Hartmann, 2011). These extratropical effects occur primarily in winter when the stratosphere and troposphere are strongly coupled (Anstey and Shepherd, 2008; Garfinkel and Hartmann, 2011).

It has been unclear how the $\mathrm{QBO}$ will respond to future climate change related to GHG increase and recovery of stratospheric ozone. Climate models assessed in the AR4 did not simulate the QBO as they lacked the necessary vertical resolution (Kawatani et al., 2011). Recent model studies without using gravity wave parameterization (Kawatani et al., 2011; Kawatani et al., 2012) showed that the QBO period and amplitude may become longer and weaker, and the downward penetration into the lowermost stratosphere may be more curtailed in a warmer climate. This finding is attributed to the effect of increased equatorial upwelling (stronger Brewer-Dobson circulation; Butchart et al., 2006; Garcia and Randel, 2008; McLandress and Shepherd, 2009; Okamoto et al., 2011) dominating the effect of increased wave forcing (more convective activity). Two studies with gravity wave parameterization, however, gave conflicting results depending on the simulated changes in the intensity of the Brewer-Dobson circulation (Watanabe and Kawatani, 2012).
There are limited published results on the future behaviour of the QBO, using CMIP5 models. On the basis of the recent literature, it is uncertain how the period or amplitude of the QBO may change in future and confidence in the projections remains low.

\subsubsection{Atlantic Multi-decadal Oscillation}

The AMO (Box 2.5; see also Section 9.5.3.3.2) is a fluctuation seen in the instrumental SST record throughout the North Atlantic Ocean and is related to variability in the thermohaline circulation (Knight et al., 2005). Area-mean North Atlantic SST shows variations with a range of about $0.4^{\circ} \mathrm{C}$ (see Box 2.5) and a warming of a similar magnitude since 1870. The AMO has a quasi-periodicity of about 70 years, although the approximately 150-year instrumental record possesses only a few distinct phases-warm during approximately 1930-1965 and after 1995, and cool between 1900-1930 and 1965-1995. The phenomenon has also been referred to as 'Atlantic Multidecadal Variability' to avoid the implication of temporal regularity. Along with secular trends and Pacific variability, the AMO is one of the principal features of multidecadal variability in the instrumental climate record.

The AR4 highlighted a number of important links between the AMO and regional climates. Subsequent research using observational and paleoclimate records, and climate models, has confirmed and expanded upon these connections, such as West African monsoon and Sahel rainfall (Mohino et al., 2011; Section 14.2.4), summer climate in North America (Seager et al., 2008; Section 14.8.3; Feng et al., 2011) and Europe (Folland et al., 2009; Ionita et al., 2012; Section 14.8.6), the Arctic (Chylek et al., 2009; Mahajan et al., 2011), and Atlantic major hurricane frequency (Chylek and Lesins, 2008; Zhang and Delworth, 2009; Section 14.6.1). Further, the list of AMO influences around the globe has been extended to include decadal variations in many other regions (e.g., Zhang and Delworth, 2006; Kucharski et al., 2009a, 2009b; Huss et al., 2010; Marullo et al., 2011; Wang et al., 2011).

Paleo reconstructions of Atlantic temperatures show AMO-like variability well before the instrumental era, as noted in the AR4 (Chapter 6; see also Section 5.4.2). Recent analyses confirm this, and suggest potential for intermittency in AMO variability (Saenger et al., 2009; Zanchettin et al., 2010; Chylek et al., 2012). Control simulations of climate models run for hundreds or thousands of years also show long-lived Atlantic multi-decadal variability (Menary et al., 2012). These lines of evidence suggest that AMO variability will continue into the future. No fundamental changes in the characteristics of North Atlantic multi-decadal variability in the 21st century are seen in CMIP3 models (Ting et al., 2011).

Many studies have diagnosed a trend towards a warm North Atlantic in recent decades additional to that implied by global climate forcings (Knight, 2009; Polyakov et al., 2010). It is unclear exactly when the current warm phase of the AMO will terminate, but may occur within the next few decades, leading to a cooling influence in the North Atlantic and offsetting some of the effects characterizing global warming (Keenlyside et al., 2008; see also Section 11.3.3.3).

Some similarity in the shape of the instrumental time series of global and $\mathrm{NH}$ mean surface temperatures and the AMO has long been noted. By removing an estimate of the effect of interannual variability phe- 
nomena like ENSO (Section 14.4), AMO transitions have been shown to have the potential to produce large and abrupt changes in hemispheric temperatures (Thompson et al., 2010). Estimates of the AMO's contribution to recent climate change are uncertain, however, as attribution of the observed AMO requires a model (physical or conceptual) whose assumptions are nearly always difficult to verify (Knight, 2009).

\subsubsection{Assessment Summary}

Literature is generally insufficient to assess future changes in behaviour of the PNA, PSA, TBO, QBO and PDO/IPO. Confidence in the projections of changes in these modes is therefore low. However, process understanding of the formation of the PSA gives medium confidence that future changes in the mean atmospheric circulation for austral summer will project on the PSA pattern thereby influencing the SACZ and precipitation over Southeastern South America.

Paleoclimate reconstructions and long model simulations indicate that the AMO is unlikely to change its behaviour in the future as the mean climate changes. However, natural fluctuations in the AMO over the coming few decades are likely to influence regional climates at least as strongly as will human-induced changes.

\subsection{Future Regional Climate Change}

\subsubsection{Overview}

The following sections assess future climate projections for several regions, and relate them, where possible, to projected changes in the major climate phenomena assessed in Sections 14.2 to 14.7. The regional climate change assessments are mainly of mean surface air temperature and mean precipitation based primarily on multi-model ensemble projections from general circulation models. Reference is made to the appropriate projection maps from CMIP5 (Taylor et al., 2011c) presented in Annex I: Atlas of Global and Regional Climate Projections. Annex I uses smaller sub-regions similar to those introduced by SREX (Seneviratne et al., 2012). Table 14.1 presents a quantitative summary of the regional area averages over three projection periods (2016-2035, 2046-2065 and 2081-2100 with respect to the reference period 19862005 , representing near future, middle century and end of century) for the RCP4.5 scenario. The 26 land regions assessed here are presented in Seneviratne et al., 2012, page 12 and the coordinates can be found from their online Appendix 3.A. Added to this are six additional regions containing the two polar regions, the Caribbean, Indian Ocean and Pacific Island States (see Annex I for further details). Table 14.1 identifies the smaller sub-domains grouped within the somewhat large regions that are discussed in Sections 14.8.2 to 14.8.15. Tables for RCP2.6, RCP6.0 and RCP8.5 scenarios are presented in Supplementary Material Tables 14.SM.1a to 14.SM.1c. For continental-scale regions, projected changes in mean precipitation between (2081-2100) and (1986-2005) are compared in two generations of models forced under two comparable emission scenarios: RCP4.5 in CMIP5 versus A1B in CMIP3. In contrast to the Annex, the seasons here are chosen differently for each region so as to best capture the regional features such as monsoons. Downscaling issues are illustrated in panels showing results from an ensemble of high-resolution time-slice experiments with the Meteorological
Research Institute (MRI) model (Endo et al., 2012; Mizuta et al., 2012). To facilitate a direct comparison across the scenarios, the precipitation changes are normalized by the global annual mean surface air temperature changes in each scenario. Published results using other downscaling methods are also assessed when found essential to illustrate issues related to regional climate change.

Regional climate projections are generally more uncertain than projections of global mean temperature but the sources of uncertainty are similar (see Chapters 8, 11, and 12) yet differ in relative importance. For example, natural variability (Deser et al., 2012), aerosol forcing (Chapter 7) and land use/cover changes (DeFries et al., 2002; Moss et al., 2010) all become more important sources of uncertainty on a regional scale. Regional climate assessments incur additional uncertainty due to the cascade of uncertainty through the hierarchy of models needed to generate local information (cf. downscaling in Section 9.6). Calibration (bias correction) of model output to match local observations is an additional important source of uncertainty in regional climate projections (e.g., Ho et al., 2012), which should be considered when interpreting the regional projections. Therefore, the model spread shown in Annex 1 should not be interpreted as the final uncertainty in the observable regional climate change response.

Table 14.2 summarizes the assessed confidence in the ability of CMIP5 models to represent regional scale present-day climate (temperature and precipitation, based on Chapter 9), the main controlling phenomena for weather and climate in that region and the assessed resulting confidence in the future projections. There is generally less confidence in projections of precipitation than of temperature. For example, in Annex I, the temperature projections for 2081-2100 are almost always above the model estimates of natural variability, whereas the precipitation projections less frequently rise above natural variability. Although some projections are robust for reasons that are well understood (e.g., the projected increase in precipitation at high latitudes), many other regions have precipitation projections that vary in sign and magnitude across the models. These issues are further discussed in Section 12.4.5.2. Details on how the confidence table is constructed are found in the Supplementary Material.

Credibility in regional climate change projections is increased if it is possible to find key drivers of the change that are known to be well-simulated and well-projected by climate models. Table 14.3 summarizes the assessment of how major climate phenomena might be relevant for future regional climate change. For each entry in the table, the relevance is based on an assessment of confidence in future change in the phenomenon and the confidence in how the phenomenon influences regional climate. For example, NAO is assigned high relevance (red) for the Arctic region because NAO is known to influence the Arctic and there is high confidence that the NAO index will increase in response to anthropogenic forcing. If there is low confidence in how a phenomenon might change (e.g., ENSO) but high confidence that it has a strong regional impact, then the cell in the table is assigned medium relevance (yellow). It can be seen from the table that there are many cases where major phenomena are assessed to have high (red) or medium (yellow) relevance for future regional climate change. See Supplementary Material Section 14.SM.6.1 for more details on how this relevance table was constructed. 
Frequently Asked Questions

\section{FAQ 14.2 | How Are Future Projections in Regional Climate Related to Projections of Global Means?}

The relationship between regional climate change and global mean change is complex. Regional climates vary strongly with location and so respond differently to changes in global-scale influences. The global mean change is, in effect, a convenient summary of many diverse regional climate responses.

Heat and moisture, and changes in them, are not evenly distributed across the globe for several reasons:

- External forcings vary spatially (e.g., solar radiation depends on latitude, aerosol emissions have local sources, land use changes regionally, etc.).

- Surface conditions vary spatially, for example, land/sea contrast, topography, sea surface temperatures, soil moisture content.

- Weather systems and ocean currents redistribute heat and moisture from one region to another.

Weather systems are associated with regionally important climate phenomena such as monsoons, tropical convergence zones, storm tracks and important modes of climate variability (e.g., El Niño-Southern Oscillation (ENSO), North Atlantic Oscillation (NAO), Southern Annular Mode (SAM), etc.). In addition to modulating regional warming, some climate phenomena are also projected to change in the future, which can lead to further impacts on regional climates (see Table 14.3).

Projections of change in surface temperature and precipitation show large regional variations (FAQ 14.2, Figure 1). Enhanced surface warming is projected to occur over the high-latitude continental regions and the Arctic ocean,

(continued on next page)
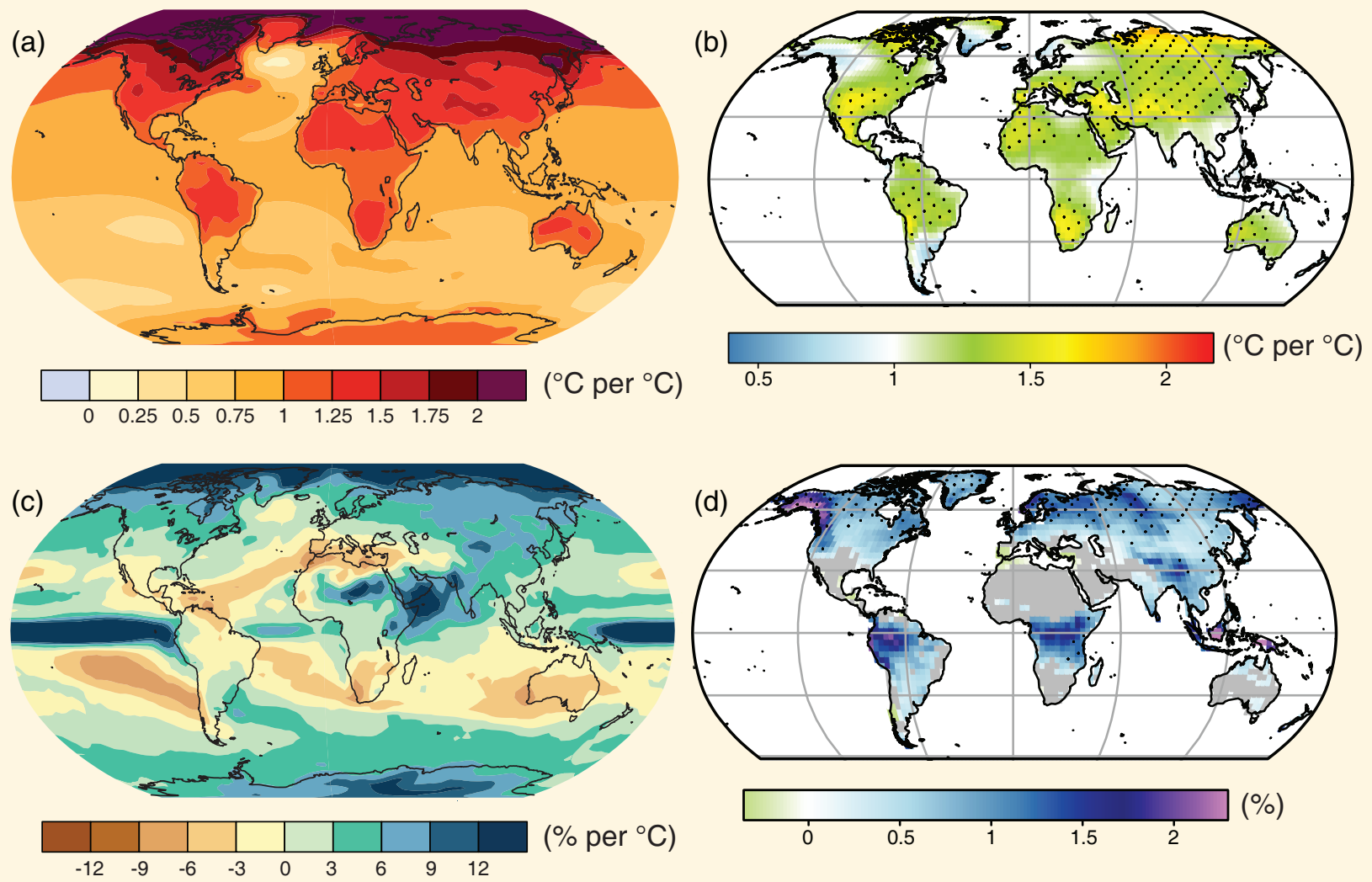

FAQ 14.2, Figure 1 | Projected 21st century changes in annual mean and annual extremes (over land) of surface air temperature and precipitation: (a) mean surface temperature per ${ }^{\circ} \mathrm{C}$ of global mean change, (b) 90 th percentile of daily maximum temperature per ${ }^{\circ} \mathrm{C}$ of global average maximum temperature, (c) mean precipitation (in $\%$ per ${ }^{\circ} \mathrm{C}$ of global mean temperature change), and (d) fraction of days with precipitation exceeding the 95th percentile. Sources: Panels (a) and (c) projected changes in means between 1986-2005 and 2081-2100 from CMIP5 simulations under RCP4.5 scenario (see Chapter 12, Figure 12.41); Panels (b) and (d) projected changes in extremes over land between 1980-1999 and 2081-2100 (adapted from Figures 7 and 12 of Orlowsky and Seneviratne, 2012). 


\section{FAQ 14.2 (continued)}

while over other oceans and lower latitudes changes are closer to the global mean (FAQ 14.2, Figure 1a). For example, warming near the Great Lakes area of North America is projected to be about $50 \%$ greater than that of the global mean warming. Similar large regional variations are also seen in the projected changes of more extreme temperatures (FAQ 14.2, Figure 1b). Projected changes in precipitation are even more regionally variable than changes in temperature (FAQ 14.2, Figure 1c, d), caused by modulation from climate phenomena such as the monsoons and tropical convergence zones. Near-equatorial latitudes are projected to have increased mean precipitation, while regions on the poleward edges of the subtropics are projected to have reduced mean precipitation. Higher latitude regions are projected to have increased mean precipitation and in particular more extreme precipitation from extratropical cyclones.

Polar regions illustrate the complexity of processes involved in regional climate change. Arctic warming is projected to increase more than the global mean, mostly because the melting of ice and snow produces a regional feedback by allowing more heat from the Sun to be absorbed. This gives rise to further warming, which encourages more melting of ice and snow. However, the projected warming over the Antarctic continent and surrounding oceans is less marked in part due to a stronger positive trend in the Southern Annular Mode. Westerly winds over the midlatitude southern oceans have increased over recent decades, driven by the combined effect of loss of stratospheric ozone over Antarctica, and changes in the atmosphere's temperature structure related to increased greenhouse gas concentrations. This change in the Southern Annular Mode is well captured by climate models and has the effect of reducing atmospheric heat transport to the Antarctic continent. Nevertheless, the Antarctic Peninsula is still warming rapidly, because it extends far enough northwards to be influenced by the warm air masses of the westerly wind belt.

\subsubsection{Arctic}

This section is concerned with temperature and precipitation dimensions of Arctic climate change, and their links to climate phenomena. The reader is referred elsewhere for information on sea ice loss (Sections 4.2.2, 5.5.2 and Chapter 10), and projections of sea ice change (Sections 9.4.3, 9.8.3 and Chapters 11 and 12).

Arctic climate is affected by three modes of variability: NAO (Section 14.5.1), PDO (Section 14.7.3) and AMO (Section 14.7.6). The NAO index correlates positively with temperatures in the northeastern Eurasian sector, and correlates negatively with temperatures in the Baffin Bay and Canadian Archipelago, but exhibits little relationship with central Arctic temperatures (Polyakov et al., 2003). The PDO plays a role in temperature variability of Alaska and the Yukon (Hartmann and Wendler, 2005). The AMO is positively associated with SST throughout the Arctic (Chylek et al., 2009; Levitus et al., 2009; Chylek et al., 2010) (Mahajan et al., 2011). ETCs are also mainly responsible for winter precipitation in the region (see Table 14.3).

The surface and lower troposphere in the Arctic and surrounding land areas show regional warming over the past three decades of about $1{ }^{\circ} \mathrm{C}$ per decade - significantly greater than the global mean trend (Figures 2.22 and 2.25). According to temperature reconstructions, this signal is highly unusual: Temperatures averaged over the Arctic over the past few decades are significantly higher than any seen over the past 2000 years (Kaufman et al., 2009). Temperatures 11 ka were greater than the 20th century mean, but this is probably a strongly forced signal, since summer solar radiation was $9 \%$ greater than present (Miller et al.,
2010). Finally, warmer temperatures have been sustained in pan-Arctic land areas where a declining NAO over the past decade ought to have caused cooling (Semenov, 2007; Turner et al., 2007b). Since AR4, evidence has also emerged that precipitation has trended upward in most pan-Arctic land areas over the past few decades (e.g., Pavelsky and Smith, 2006; Rawlins et al., 2010), though the evidence remains mixed (e.g., Dai et al., 2009). Increasing ETC activity over the Canadian Arctic has also been observed (Section 2.6.4).

Since AR4, there has been progress in adapting RCMs for polar applications (Wilson et al., 2012). These models have been evaluated with regard to their ability to simulate Arctic clouds, surface heat fluxes, and boundary layer processes (Tjernstrom et al., 2004; Inoue et al., 2006; Rinke et al., 2006). They have been used to improve simulations of Arctic-specific climate processes, such as glacial mass balance (Zhang et al., 2007). A few regional models have been used for Arctic climate change projections (e.g., Zahn and von Storch, 2010; Koenigk et al., 2011; Döscher and Koenigk, 2012). For information on GCM quality in the Arctic, see Chapter 9 and the brief summary of assessed confidence in the CMIP5 models in Table 14.2.

The CMIP5 model simulations exhibit an ensemble-mean polar amplified warming, especially in winter, similar to CMIP3 model simulations (Bracegirdle and Stephenson, 2012; see also Box 5.1). For RCP4.5, ensemble-mean winter warming rises to $5.0^{\circ} \mathrm{C}$ over pan-Arctic land areas by the end of the 21 st century $(2081-2100)$, and about $7.0^{\circ} \mathrm{C}$ over the Arctic Sea (Table14.1). Throughout the century, the warming exceeds simulated estimates of internal variability (Figure Al.8). The RCP4.5 ensemble-mean warming is more modest in JJA (Table 14.1), 
reaching about $2.2^{\circ} \mathrm{C}$ by century's end over pan-Arctic land areas, and $1.5^{\circ} \mathrm{C}$ over the Arctic Sea. The summer warming exceeds variability estimates by about mid-century (Figure Al.9). These simulated anthropogenic seasonal warming patterns match qualitatively the observed warming patterns over the past six decades (AMAP, 2011), and the observed warming patterns are likely to be at least partly anthropogenic in origin (Section 10.3.1.1.4). Given the magnitude of future projected changes relative to variability, and the presence of anthropogenic signals already, it is likely future Arctic surface temperature changes will continue to be strongly influenced by the anthropogenic forcing over the coming decades.

The CMIP5 models robustly project precipitation increases in the pan-Arctic (both land and sea) region over the 21st century, as did their CMIP3 counterparts (Kattsov et al., 2007; Rawlins et al., 2010). Under the RCP4.5 scenario, the cold season, ensemble mean precipitation increases about $25 \%$ by the century's end (Table 14.1), due to enhanced precipitation in ETCs (Table 14.3). However, this signal does not rise consistently above the noise of simulated variability until mid $21^{\text {st }}$ century (Figure Al.10). During the warm season, precipitation increases are smaller, about 15\% (Table 14.1), though these signals also rise above variability by mid $21^{\text {st }}$ century (Figure Al.11). The inter-model spread in the precipitation increase is generally as large as the ensemble mean signal itself (similar to CMIP3 model behaviour, Holland and Webster, 2007), so the magnitude of the future increase is uncertain. However, since nearly all models project a large precipitation increase rising above the variability year-round, it is likely the pan-Arctic region will experience a statistically significant increase in precipitation by mid-century (see also Table 14.2). The small projected increase in the NAO is likely to affect Arctic precipitation (and temperature) patterns in the coming century (Section 14.5.1; Table 14.3), though the importance of these signals relative to anthropogenic signals described here is unclear.

In summary: It is likely Arctic surface temperature changes will be strongly influenced by anthropogenic forcing over the coming decades dominating natural variability such as induced by NAO. It is likely the pan-Arctic region will experience a significant increase in precipitation by mid-century due mostly to enhanced precipitation in ETCs.

\subsubsection{North America}

The climate of North America (NA) is affected by the following phenomena: NAO (Section 14.5.1), ENSO (Section 14.4), PNA (Section 14.7.2), PDO (Section 14.7.3), NAMS (Section 14.2.3), TCs and ETCs (Section 14.6). The NAO affects temperature and precipitation over Eastern NA during winter (Hurrell et al., 2003). Positive PNA brings warmer temperatures to northern Western NA and Alaska in winter, cooler temperatures to the southern part of Eastern NA, and dry conditions to much of Eastern NA (Nigam, 2003). The PNA can also be excited by ENSO-related SST anomalies (Horel and Wallace, 1981; Nigam, 2003). The PDO is linked to decadal climate anomalies resembling those of the PNA. The NAMS brings excess summer rainfall to Central America and Mexico and the southern portion of Western NA (Gutzler, 2004). TCs also impact the Gulf Coast and Eastern NA (see Section 14.6.1). The AMM and AMO may affect their frequency and intensity (Landsea et al., 1999; Goldenberg et al., 2001; Cassou et al., 2007; Emanuel,
2007; Vimont and Kossin, 2007; Smirnov and Vimont, 2011). ETCs are also mainly responsible for winter precipitation, especially in the northern half of NA. See Table 14.3 for a summary of this information.

A general surface warming over NA has been documented over the last century (see Section 2.4). It is particularly large over Alaska and northern Western NA during winter and spring and the northern part of Eastern NA during summer (Zhang et al., 2011b). There is also a cooling tendency over Central and Eastern NA (i.e., the 'warming hole' discussed in Section 2.4.1) during spring, though it is absent in lower tropospheric temperature (cf. Figure 2.25). The warming has coincided with a general decline in NA snow extent and depth (Brown and Mote, 2009; McCabe and Wolock, 2010; Kapnick and Hall, 2012). Consistent with surface temperature trends, temperature extremes also exhibit secular changes. Cold days and nights have decreased in the last half century, while warm days and nights have increased (see Chapter 2). These changes are especially apparent for nightly extremes (Vincent et al., 2007). It is unclear whether there have been mean precipitation trends over the last 50 years (Section 2.5.1; Zhang et al., 2011b). However, precipitation extremes increased, especially over Central and Eastern NA (see Section 2.6.2 and Seneviratne et al., 2012).

Table 14.2 provides an assessment of GCM quality for simulations of temperature, precipitation, and main phenomena in NA's regions. Regarding regional modelling experiments since AR4, biases have decreased somewhat as resolutions increase. The North American Regional Climate Change Assessment Program created a simulation suite for NA at $50-\mathrm{km}$ resolution. When forced by reanalyses, this suite generally reproduces climate variability within observational error (Leung and Qian, 2009; Wang et al., 2009b; Gutowski, 2010; Mearns et al., 2012).Other regional modelling experiments covering parts or all of NA have shown improvements as resolution increases (Liang et al., 2008a; Lim et al., 2011; Yeung et al., 2011), including for extremes (Kawazoe and Gutowski, 2013). Bias reductions are large for snowpack in topographically complex Western NA, as revealed by 2- to 20-km resolution regional simulations (Qian et al., 2010b; Salathe Jr. et al., 2010; Pavelsky et al., 2011; Rasmussen et al., 2011). Thus there has been substantial progress since AR4 in understanding the value of regional modelling in simulating NA climate. The added value of using regional models to simulate climate change is discussed in Section 9.6.6.

NA warming patterns in RCP4.5 CMIP5 projections are generally similar to those of CMIP3 (Figures Al.4 and AI.5, Table 14.1). In winter, warming is greatest in Alaska, Canada, and Greenland (Figures AI.12 and Al.16), while in summer, maximum warming shifts south, to Western, Central, and Eastern NA. Examining near-term (2016-2035) CMIP5 projections of the less sensitive models (25th percentile, i.e., upper left maps in Figures Al.12, Al.13, Al.16, Al.17, Al.20 Al.21, Al.24 and Al.25), the warming generally exceeds natural variability estimates. Exceptions are Alaska, parts of Western, Central, and Eastern NA, and Canada and Greenland during winter, when natural variability linked to wintertime storms is particularly large. By 2046-2065, warming in all regions exceeds the natural variability estimate for all models. Thus it is very likely the warming signal will be large compared to natural variability in all NA regions throughout the year by mid-century. This warming generally leads to a two- to four fold increase in simulated heat wave frequency over the 21st century (e.g., Lau and Nath, 2012). 
Anthropogenic climate change may also bring systematic cold-season precipitation changes. As with previous models, CMIP5 projections generally agree in projecting a winter precipitation increase over the northern half of NA (Figure 14.18 and Al.19). This is associated with increased atmospheric moisture, increased moisture convergence, and a poleward shift in ETC activity (Section 14.6.2 and Table 14.3). The change is consistent with CMIP3 model projections of positive NAO trends (Table 14.3; Hori et al., 2007; Karpechko, 2010; Zhu and Wang, 2010). Winter precipitation increases extend southward into the USA (northern portions of SREX regions 3 to 5; Neelin et al., 2013) but with decreasing strength relative to natural variability. This behaviour is qualitatively reproduced in higher resolution simulations (Figure 14.18).

Warm-season precipitation also exhibits significant increases in Alaska, northern Canada, and Eastern NA by century's end (Figures 14.18, Al.19, Al.22). However, CMIP5 models disagree on the sign of the precipitation change over the rest of NA (Figures Al.26 and Al.27), consistent with CMIP3 results (Figure 14.18; Neelin et al., 2006; Rauscher et al., 2008; Seth et al., 2010). One set of high resolution simulatons (Endo et al., 2012) shows a tendency towards more precipitation than either CMIP3 or CMIP5 models (Figure 14.18), suggesting the simulated warm-season precipitation change in the region may be resolution-dependent. Future precipitation changes associated with the NAMS are likewise uncertain, though there is medium confidence the phenomenon will move to later in the annual cycle (Section 14.2.3, Table 14.3). As there is medium confidence tropical cyclones will be associated with greater rainfall rates, the Gulf and East coasts of NA may be impacted by greater precipitation when tropical cyclones occur (Table 14.3).

CMIP3 models showed a 21st century precipitation decrease across much of southwestern NA, accompanied by a robust evaporation increase characteristic of mid-latitude continental warming (Seager et al., 2007; Seager and Vecchi, 2010) and an increase in drought frequency (Sheffield and Wood, 2008; Gutzler and Robbins, 2011). When downscaled, CMIP3 models showed less drying in the region (Gao et al., 2012c) and an extreme precipitation increase, despite overall drying (Dominguez et al., 2012). CMIP5 models do not consistently show such a precipitation decrease in this region (Neelin et al., 2013). This is one of the few emerging differences between the two ensembles in climate projections over NA. However, the CMIP5 models still show a strong decrease in soil moisture here (Dai, 2013), due to increasing evaporation.

In summary, it is very likely that by mid-century the anthropogenic warming signal will be large compared to natural variability such as that stemming from the NAO, ENSO, PNA, PDO, and the NAMS in all
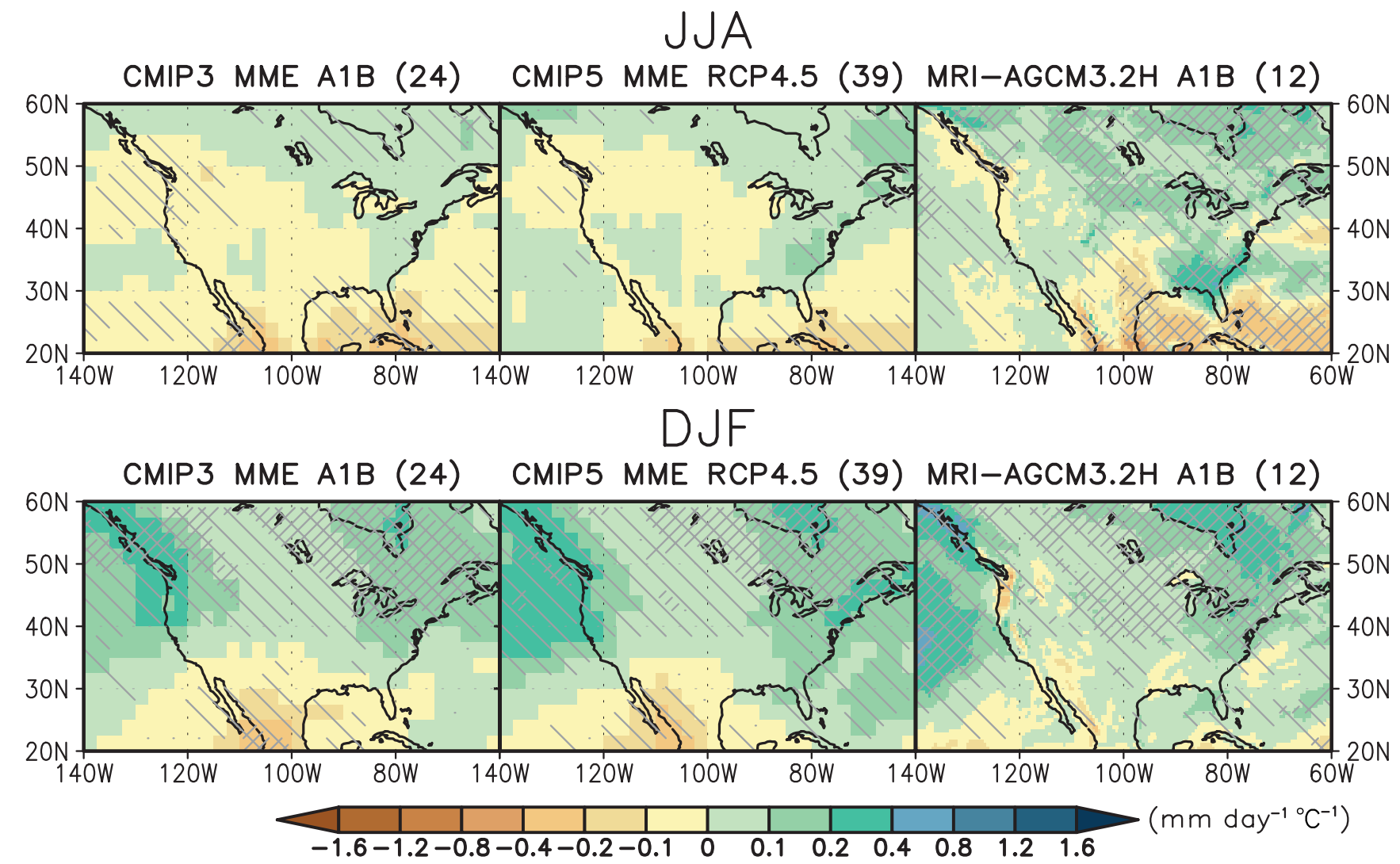

Figure 14.18 | Maps of precipitation changes for North America in 2080-2099 with respect to 1986-2005 in June, July and August (above) and December to February (below) in the SRES A1B scenario with 24 CMIP3 models (left), and in the RCP4.5 scenario with 39 CMIP5 models (middle). Right figures are the precipitation changes in $2075-2099$ with respect to 1979-2003 in the SRES A1B scenario with the 12 member 60 km mesh Meteorological Research Institute (MRI)-Atmospheric General Circulation Model 3.2 (AGCM3.2) multi-physics, multi-SST ensembles (Endo et al., 2012). Precipitation changes are normalized by the global annual mean surface air temperature changes in each scenario. Light hatching denotes where more than $66 \%$ of models (or members) have the same sign with the ensemble mean changes, while dense hatching denotes where more than $90 \%$ of models (or members) have the same sign with the ensemble mean changes. 
NA regions throughout the year. It is likely that the northern half of NA will experience an increase in precipitation over the 21st century, due in large part to a precipitation increase within ETCs.

\subsubsection{Central America and Caribbean}

The Central America and the Caribbean (CAC) region is affected by several phenomena, including the ITCZ (Section 14.3.1.1), NAMS (Section 14.2.3.1), ENSO (Section 14.4) and TCs (Section 14.6.1; Table 14.3; also Gamble and Curtis, 2008). The annual cycle results from air-sea interactions over the Western Hemisphere warm pool in the tropical eastern north Pacific and the Intra Americas Seas (Amador et al., 2006; Wang et al., 2007). The Caribbean Low Level Jet is a key element of the region's summer climate (Cook and Vizy, 2010) and is controlled by the size and intensity of the Western Hemisphere warm pool (Wang et al., 2008b). It is also modulated by SST gradients between the eastern equatorial Pacific and tropical Atlantic (Taylor et al., 2011d). ENSO is the main driver of climate variability, with El Niño being associated with dry conditions and La Niña with wet conditions (Karmalkar et al., 2011). Other teleconnection patterns, such as the NAO (Section 14.5.1) and the strength of boreal winter convection over the Amazon, influence trade winds over the Tropical North Atlantic and can combine with ENSO to modulate the summer Western Hemisphere warm pool (e.g., Enfield et al., 2006). Table 14.3 summarizes the main phenomena and their relevance to climate change over the CAC.

Because inter-decadal climate variations can be large in the CAC region, precipitation trends must be interpreted carefully. From 1950 to 2003, negative trends were seen in several data sets in the Caribbean region and parts of Central America (Neelin et al., 2006). However, regarding secular trends (1901-2005), this signal was identified only in the Caribbean region (Trenberth et al., 2007b). Prolonged dry or wet periods are related to decadal variability of the adjacent Pacific and Atlantic (Mendoza et al., 2007; Seager et al., 2009; Mendez and Magaña, 2010), and the intensity of easterlies over the region. For instance, increased easterly surface winds over Puerto Rico from 1950 to 2000 disrupted a pattern of inland moisture convergence, leading to a dramatic precipitation decrease (Comarazamy and Gonzalez, 2011).

Table 14.2 provides an overall assessment of GCM quality for simulations of temperature, precipitation and main phenomena in the CAC sub-regions. Annual cycles of temperature and precipitation are well

\section{JJAS}

CMIP3 MME A1B (24) CMIP5 MME RCP4.5 (39) MRI-AGCM3.2H A1B (12)

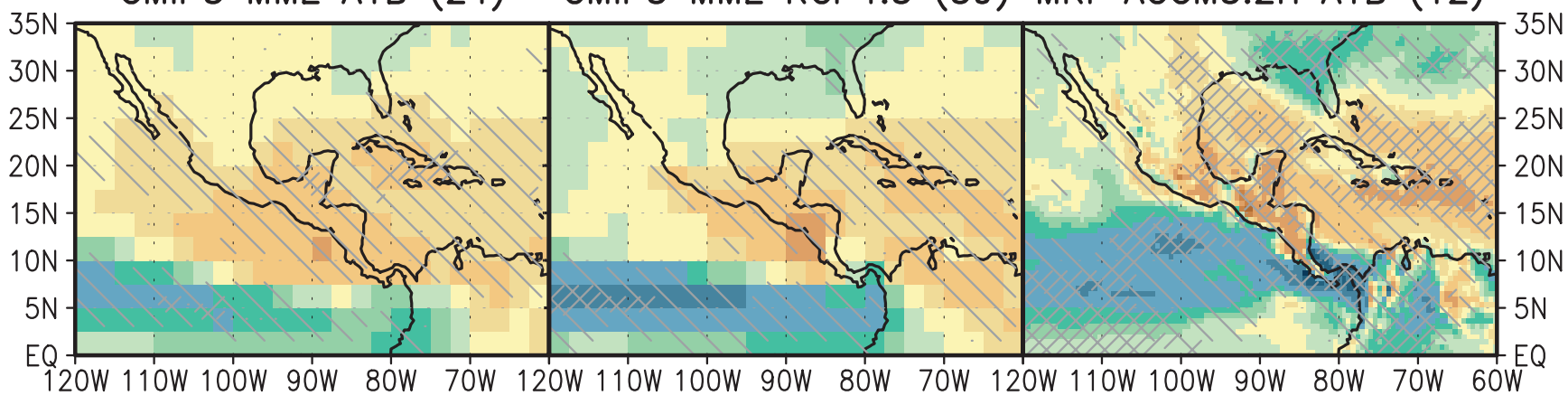

DJFM

CMIP3 MME A1B (24) CMIP5 MME RCP4.5 (39) MRI-AGCM3.2H A1B (12)
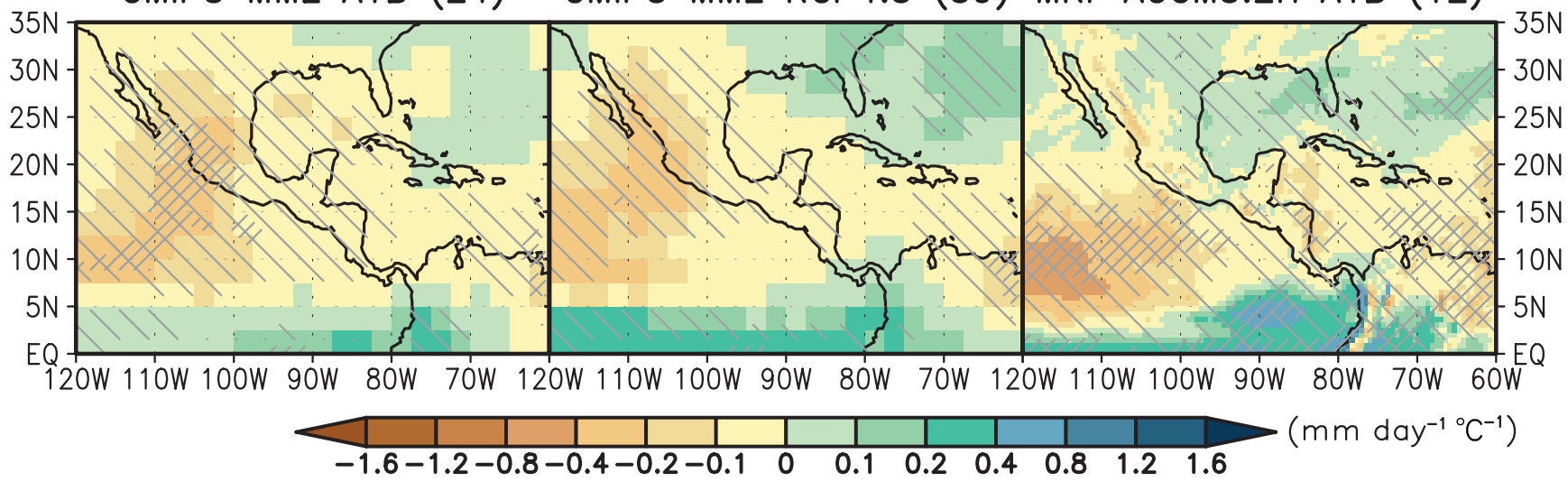

Figure 14.19 | Maps of precipitation changes for Central America and Caribbean in 2080-2099 with respect to 1986-2005 in June to September (above) and December to March (below) in the SRES A1B scenario with 24 CMIP3 models (left), and in the RCP4.5 scenario with 39 CMIP5 models (middle). Right figures are the precipitation changes in 2075-2099 with respect to 1979-2003 in the SRES A1B scenario with the 12 member 60 km mesh Meteorological Research Institute (MRI)-Atmospheric General Circulation Model 3.2 (AGCM3.2) multi-physics, multi-SST ensembles (Endo et al., 2012). Precipitation changes are normalized by the global annual mean surface air temperature changes in each scenario. Light hatching denotes where more than $66 \%$ of models (or members) have the same sign with the ensemble mean changes, while dense hatching denotes where more than $90 \%$ of models (or members) have the same sign with the ensemble mean changes. 
simulated by CMIP5 models, though precipitation from June to October is underestimated (Figure 9.38). Regional models also simulate temperature and precipitation climatologies, and the magnitude and annual cycle of the Caribbean Low-Level Jet reasonably well (Campbell et al., 2010; Taylor et al., 2013).

CMIP3 models generally projected a precipitation reduction over much of the Caribbean region, consistent with the observed negative trend since 1950 (Neelin et al., 2006; Rauscher et al., 2008). The subtropics are generally expected to dry as global climate warms (Held and Soden, 2006), but in both CMIP3 and CMIP5 models the CAC region shows the greatest drying. Future drying may also be related to strengthening of the Caribbean Low-Level Jet (Taylor et al., 2013) and subsidence over the Caribbean region associated with warmer SSTs in the tropical Pacific than Atlantic (Taylor et al., 2011d). A high-resolution regional Ocean GCM using a CMIP3 ensemble for boundary conditions confirms that the Intra American Seas circulation weakens by similar rate as the reduction in Atlantic Meridional Overturning (Liu et al., 2012c). This weakening causes the Gulf of Mexico to warm less than other oceans.

Downscaling experiments for the region have shown a mid-21st century warming between $2^{\circ} \mathrm{C}$ and $3^{\circ} \mathrm{C}$ (Vergara et al., 2007; Rauscher et al., 2008; Karmalkar et al., 2011). Precipitation decreases over most of the CAC region, similar to the signal in driving global models (Campbell et al., 2010; Hall et al., 2012). However, only a few downscaling studies took into account key elements of the region's climate, such as easterly wave activity, TCs, or interannual variability mechanisms linked to ENSO (Karmalkar et al., 2011).

By century's end, CMIP5 models project greatest warming in the CAC region in JJA. Warming is projected to be larger over Central America than the Caribbean in summer and winter (Figures AI.24, AI.2, Table 14.1). From October to March, ensemble mean projections indicate precipitation decrease in northern Central America, including Mexico. In the Caribbean precipitation is projected to decrease in the south (consistent with the observed trends) but to increase in the north (Figure Al.26). From April to September, the projected zone of precipitation reduction expands over the entire CAC region, and this signal is generally larger than the models' estimates of natural variability (Figure Al.27). Precipitation changes projected by CMIP3, CMIP5 and a high-resolution model show a similar reduction in parts of Mexico and the southern Caribbean in DJFM, and in Central America and the Caribbean in JJAS (Figure 14.19). The CMIP5 ensemble shows greater agreement in the DJFM precipitation increase in the northern Caribbean sector than CMIP3. These projected changes are also reflected in Table 14.1. Figures AI.26, AI.27 and Figure 14.19 suggest an intensification and southward displacement of the East Pacific ITCZ, which can contribute to drying in southern Central America (Karmalkar et al., 2011).

ENSO will continue to influence CAC climate, but changes in ENSO frequency or intensity remain uncertain (Section 14.4). Projected drier conditions may also be related to decreased frequency of TCs, though the associated rainfall rate of these systems are higher in future projections (Section 14.6.1).

In summary, owing to model agreement on projections and the degree of consistency with observed trends, it is likely warm-season precipitation will decrease in the Caribbean region, over the coming century. However, there is only medium confidence that Central America will experience a decrease in precipitation.

\subsubsection{South America}

South America (SA) is affected by several climate phenomena. ENSO (Section 14.4) and Atlantic Ocean modes (Section 14.3.4) have a role in interannual variability of many regions. The SAMS (Section 14.2.3.2) is responsible for rainfall over large areas, while the SACZ (Section 14.3.1.3) and Atlantic ITCZ (Section 14.3.1.1) also affect precipitation. Teleconnections such as the PSA (Section 14.7.1), the SAM (Section 14.5.2) with related ETCs (Section 14.6.2) and the IOD (Section 14.3.3) also influence climate variability. Table 14.3 summarizes the main phenomena and their assessed relevance to climate change over SA.

Positive minimum temperature trends have been observed in SA (Alexander et al., 2006; Marengo and Camargo, 2008; Rusticucci and Renom, 2008; Marengo et al., 2009; Seneviratne et al., 2012; Skansi et al., 2013). Glacial retreat in the tropical Andes was observed in the last three decades (Vuille et al., 2008; Rabatel et al., 2013). In contrast to the warming over the continental interior, a prominent but localized coastal cooling was detected during the past 30 to 50 years, extending from central Peru (Gutiérrez et al., 2011) to northern (Schulz et al., 2012) and central Chile (Falvey and Garreaud, 2009). Observed precipitation changes include a significant increase in precipitation during the 20th century over the southern sector of southeastern SA, a negative trend in SACZ continental area (Section 2.5.1; Barros et al., 2008), a negative trend in mean precipitation and precipitation extremes in central-southern Chile, and a positive trend in southern Chile (Haylock et al., 2006; Quintana and Aceituno, 2012). Other detected changes include positive extreme precipitation trends in southeastern SA, central-northern Argentina and northwestern Peru and Ecuador (Section 2.6.2; Haylock et al., 2006; Dufek et al., 2008; Marengo et al., 2009; Re and Barros, 2009; Skansi et al., 2013).

Table 14.2 provides an overall assessment of GCM quality for simulations of temperature, precipitation and main phenomena in the sub-regions of SA. In general, GCM results are consistent with observed temperature tendencies (e.g., Haylock et al., 2006). Trends toward warmer nights in CMIP3 models (Marengo et al., 2010b; Rusticucci et al., 2010) are consistent with observed trends. CMIP3 models, however, do not simulate the cooling ocean and warming land trends observed in the last 30 years along subtropical western SA noted above. The number of warm nights in SA is well represented in CMIP5 simulations (Sillmann et al., 2013). CMIP5 models reproduce the annual cycle of precipitation over SA, though the multi-model mean underestimates rainfall over some areas (Figure 9.38). In tropical SA, rainy season precipitation is better reproduced in the CMIP5 ensemble than CMIP3 (Figure 9.39). CMIP3 models were able to simulate extreme precipitation indices over SA (Rusticucci et al., 2010), but CMIP5 models improved them globally (Sillmann et al., 2013). CMIP5 also improved simulations of precipitation indices in the SAMS region (Kitoh et al., 2013; Section 14.2.3.2). The main precipitation features are well represented by regional models in several areas of SA (Solman et al., 2008; Alves and Marengo, 2010; Chou et al., 2012; Solman et al., 2013). However, regional models underestimate daily precipitation intensity in the La 
Plata Basin and in eastern Northeastern SA in DJF and almost over the whole continent in JJA (Carril et al., 2012).

Regarding future projections, CMIP5 models indicate higher temperatures over all of $\mathrm{SA}$, with the largest changes in southeastern Amazonia by century's end. (Figures Al.28, Al.29, Table 14.1). Temperature changes projected by RCMs forced by a suite of CMIP3 models agree that the largest warming occurs over the southern Amazon during austral winter. Regional models project a greater frequency of warm nights over SA, except in parts of Argentina, and a reduction of cold nights over the whole continent (Marengo et al., 2009). CMIP5 projections confirm the results of CMIP3 in AR4 and SREX (see Section 12.4.9).

CMIP5 results confirm precipitation changes projected by CMIP3 models in the majority of SA regions, with increased confidence, as more models agree in the changes (AI.30, AI.34, AI31, AI35). Inter-model spread in precipitation also decreased in some SA regions from CMIP3 to CMIP5 (Blázquez and Nuñez, 2012). CMIP5 precipitation

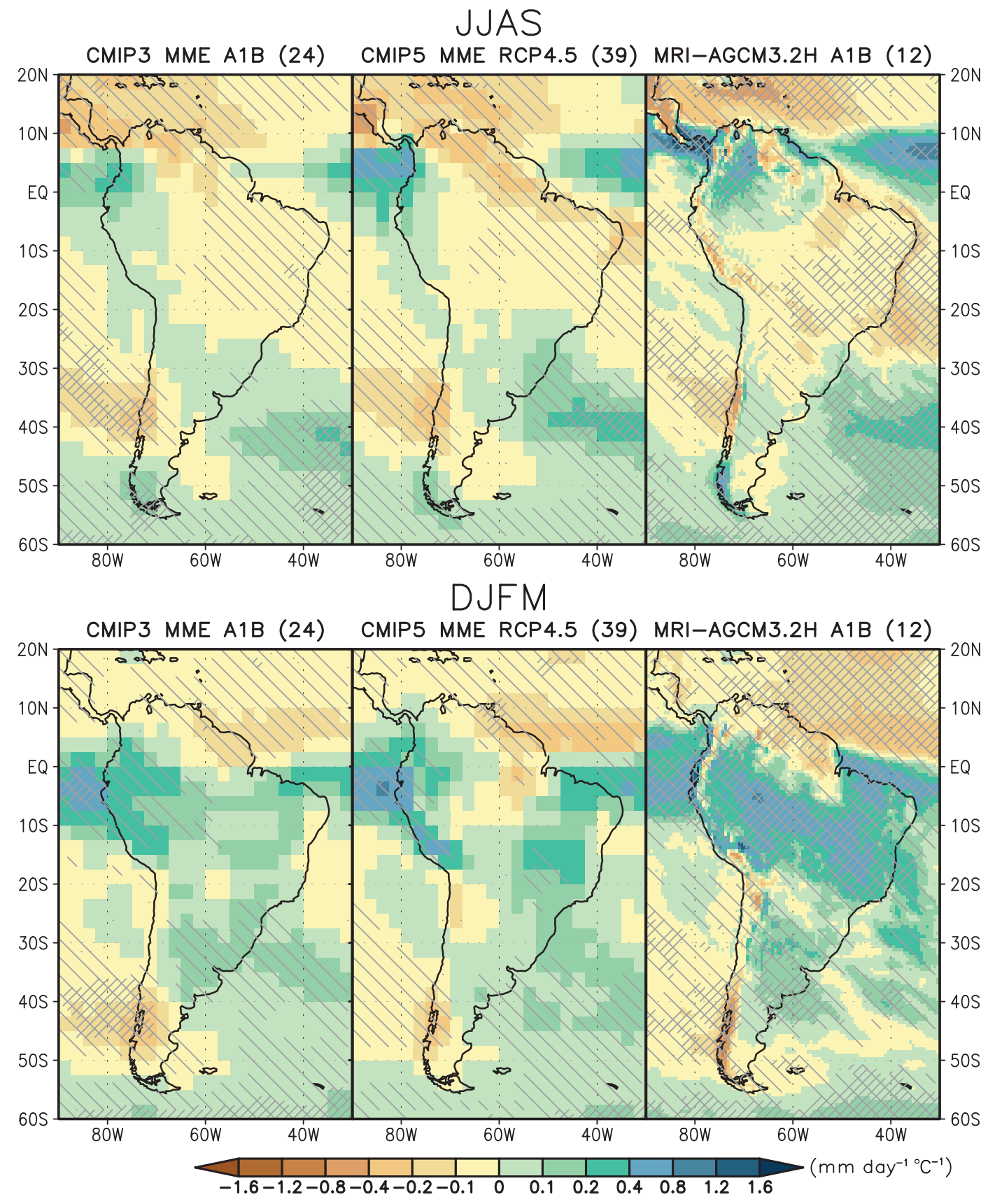

Figure 14.20 Maps of precipitation changes for South America in 2080-2099 with respect to 1986-2005 in June to September (above) and December to March (below) in the SRES A1B scenario with 24 CMIP3 models (left), and in the RCP4.5 scenario with 39 CMIP5 models (middle). Right figures are the precipitation changes in 2075-2099 with respect to 1979-2003 in the SRES A1B scenario with the 12-member 60-km mesh Meteorological Research Institute (MRI)- Atmospheric General Circulation Model 3.2 (AGCM3.2) multiphysics, multi-SST ensembles (Endo et al., 2012). Precipitation changes are normalized by the global annual mean surface air temperature changes in each scenario. Light hatching denotes where more than $66 \%$ of models (or members) have the same sign with the ensemble mean changes, while dense hatching denotes where more than $90 \%$ of models (or members) have the same sign with the ensemble mean changes. 
(a)

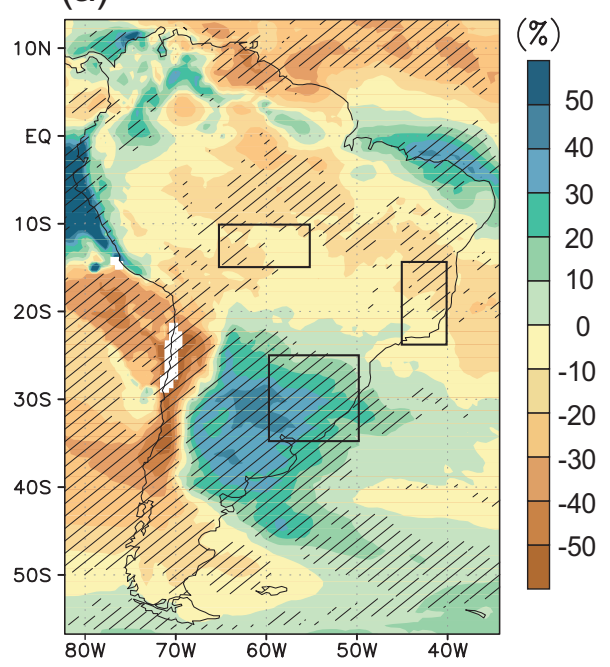

\section{Precipitation change A1B DJF}

(c)

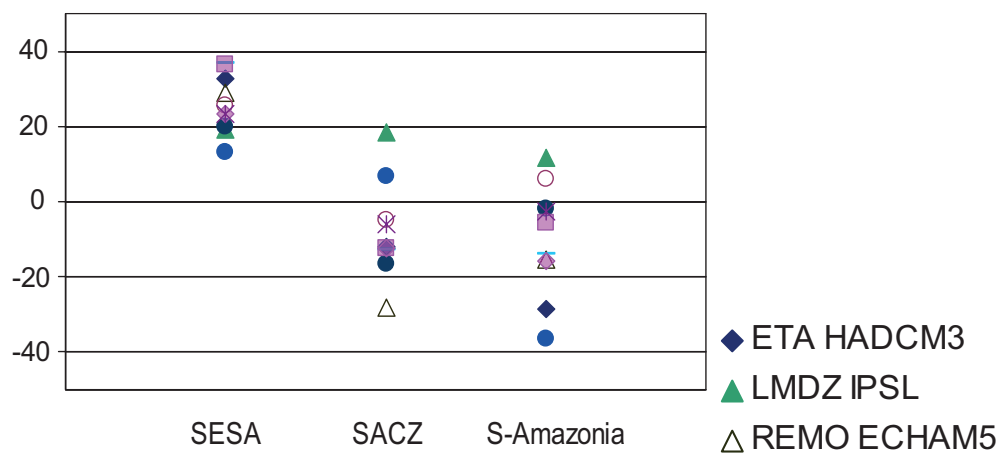

$*$ PROMES HADCM3

- REGCM3 ECHAM5

- RECGM3 HADCM3

- RCA ECHAM5 1
- RCA ECHAM5 2

$\triangle$ RCA ECHAM5 3

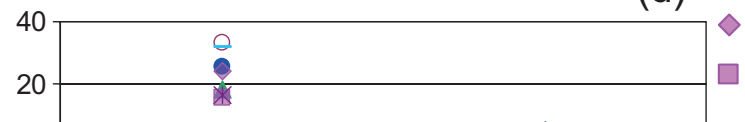

LMDZ ECHAM5

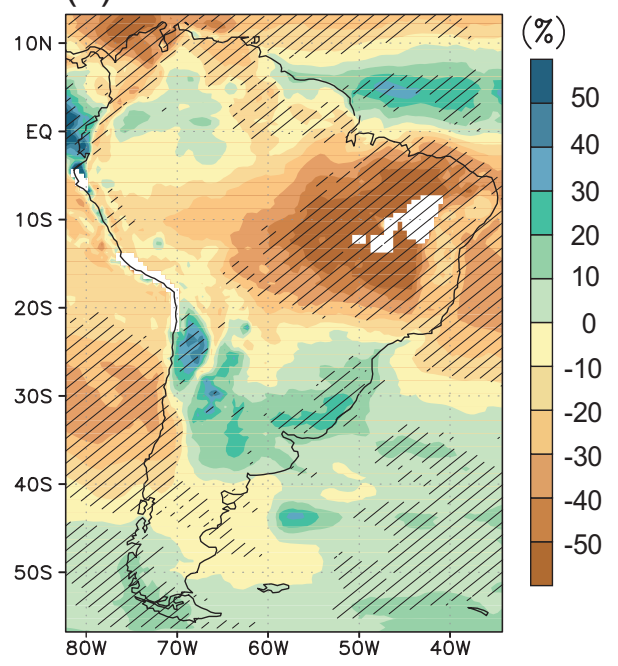

Figure 14.21 | (a) December, January and February (DJF) and (b) June, July and August (JJA) relative precipitation change in 2071-2100 with respect to 1961-1990 in the A1B scenario from an ensemble of 10 Regional Climate Models (RCMs) participating in the Europe-South America Network for Climate Change Assessment and Impact Studies-La Plata Basin (CLARIS-LPB) Project. Hatching denotes areas where 8 out of 10 RCMs agree in the sign of the relative change. (c) DJF and (d) JJA dispersion among regional model projections of precipitation changes averaged over land grid points in Southeastern South America (SESA, $35^{\circ} \mathrm{S}$ to $25^{\circ} \mathrm{S}, 60^{\circ} \mathrm{W}$ to $50^{\circ} \mathrm{W}$ ), South Atlantic Convergence Zone (SACZ, $25^{\circ} \mathrm{S}$ to $15^{\circ} \mathrm{S}, 45^{\circ} \mathrm{W}$ to $40^{\circ} \mathrm{W}$ ) and southern Amazonia ( $15^{\circ} \mathrm{S}$ to $10^{\circ} \mathrm{S}, 65^{\circ} \mathrm{W}$ to $55^{\circ} \mathrm{W}$ ), indicated by the boxes in (a).

projections for the end of the twenty-first century (2081-2100) show a precipitation increase from October to March over the southern part of Southeast Brazil and the La Plata Basin, the extreme south of Chile, the northwest coast of SA, and the Atlantic ITCZ, extending to a small area of the northeastern Brazil coast (Figures Al.30 and Al.34). Reduced October to March rainfall is projected in the extreme northern region of SA, eastern Brazil, and central Chile. In eastern Amazonia and northeastern Brazil, CMIP5 models show both drying and moistening. This uncertainty can also be seen in Table 14.1.

Figure 14.20 confirms that changes in northwestern, southwestern and southeastern SA are consistent among CMIP3 and CMIP5 ensembles and a high-resolution model ensemble, which gives more confidence in these results. However, in eastern Amazonia and northeast and eastern
Brazil, there is less agreement. Results from high-resolution or regional models forced by CMIP3 models provides further indication the projected changes are robust. A high-resolution regional model ensemble projects precipitation increases during austral summer over the La Plata Basin region, northwestern SA and southernmost Chile, and a decrease over northern SA, eastern Amazonia, eastern Brazil, central Chile and the Altiplano (Figure 14.21). Other regional models also project a precipitation increase over the Peruvian coast and Ecuador and a reduction in the Amazon Basin (Marengo et al., 2010b; Marengo et al., 2012).

From April to September, the CMIP5 ensemble projects precipitation increases over the La Plata Basin and northwestern SA near the coast (Figures Al31, Al35). In contrast, a reduction is projected for northeast 
Brazil and eastern Amazonia. Precipitation is projected to decrease in Central Chile, but to increase over extreme southern areas. In CMIP3 models, a precipitation reduction in the central Andes resulted from a moisture transport decrease from the continental interior to the Altiplano (Minvielle and Garreaud, 2011). CMIP3 and CMIP5 models are consistent in projecting drier conditions in eastern Amazonia during the dry season and wetter conditions in western Amazonia (Malhi et al., 2008; Cook et al., 2011). The Amazon forest's future is discussed in Section 12.5.5.6.1. Areas of maximum change in CMIP5 are consistent with those of CMIP3 in JJA, agreeing also with a high-resolution model ensemble (Figure 14.20). Increased precipitation in southeastern SA is projected by a high-resolution model ensemble in all four seasons (Blázquez et al., 2012). The austral winter precipitation increase over the La Plata Basin and southern Chile, and the reduction in eastern Amazonia and northeast Brazil, are also projected by RCMs (Figure 14.21) as in CMIP5 models. A relevant result from a RCM is the precipitation decrease over most of SA north of $20^{\circ} \mathrm{S}$ in austral spring, suggesting a longer dry season (Sörensson et al., 2010; see also Section 14.2.3.2). Note that average CMIP5 spatial values in Table 14.1 are consistent with changes seen in the maps, unless for the west coast of $\mathrm{SA}$, where there are spatial variations within the area and the values do not reflect the changes.

Regional model projections and a high-resolution model ensemble indicate an increase in the number of consecutive dry days in northeastern SA (Marengo et al., 2009; Kitoh et al., 2011). An increase in heavy precipitation events over almost the entire continent, especially Amazonia, southern Brazil and northern Argentina, is projected by a high-resolution model ensemble (Kitoh et al., 2011) and in subtropical areas of South America by regional models (Marengo et al., 2009). Seneviratne et al. (2012) indicated low to medium confidence in CMIP3 SA precipitation trends. However, the increased ability of CMIP5 models to represent extremes (Kitoh et al., 2013) provides higher confidence in the signals discussed above (Section 14.2.3.2), consistent with global changes in land areas (Section 12.4.5).

Precipitation changes projected over SA are consistent with El Niño influences, for example, rainfall increase over southeastern and northwestern SA and decrease over eastern Amazonia. However, CMIP3 models could not represent certain features of ENSO well (Roxy et al., 2013) and there is no consensus about future ENSO behaviour (Coelho and Goddard, 2009; Collins et al., 2010) even with CMIP5 results (Section 14.4). As the various types of ENSO produce different impacts on SA (Ashok et al., 2007; Hill et al., 2011; Tedeschi et al., 2013), future ENSO effects remain uncertain. It is very likely that ENSO remains the dominant mode of interannual variability in the future (Section 14.4.2). Therefore, regions in SA currently influenced by Pacific SST will continue to experience ENSO effects on precipitation and temperature.

Projected precipitation increases in the southern sector of southeastern SA are consistent with changes in the SACZ dipole (Section 14.3.1.3) and PSA (Section 14.7.1). Increased precipitation in this region may also have a contribution from a more frequent and intense Low Level Jet (Nuñez et al., 2009; Soares and Marengo, 2009). CMIP3 model analyses show little impact on extreme precipitation from SAM changes toward century's end, except in Patagonia (Menendez and Carril, 2010). However, the southward shift of stormtracks associated with the SAM's projected positive trend (Reboita et al., 2009; Section 14.5.2) impacts zones of cyclogenesis off the southeast SA coast (Kruger et al., 2011; Section 12.4.4).

In summary, it is very likely temperatures will increase over the whole continent, with greatest warming projected in southern Amazonia. It is likely there will be an increase (reduction) in frequency of warm (cold) nights in most regions. It is very likely precipitation will increase in the southern sector of southeastern and northwestern SA, and decrease in Central Chile and extreme north of the continent. It is very likely that less rainfall will occur in eastern Amazonia, northeast and eastern Brazil during the dry season. However, in the rainy season there is medium confidence in the precipitation changes over these regions. There is high confidence in an increase of precipitation extremes.

\subsubsection{Europe and Mediterranean}

This section assesses regional climate change in Europe and the North African and West Asian rims of the Mediterranean basin. Area-average summaries are presented for the three sub-regions of Northern Europe (NEU), Central Europe (CEU) and Mediterranean (MED) (cf. Tables 14.1 to 14.2$)$.

The most relevant climate phenomena for this region are NAO (Section 14.5.1), ETCs (Section 14.6.2) and blocking (Box 14.2, Folland et al., 2009; Feliks et al., 2010; Dole et al., 2011; Mariotti and Dell'Aquila, 2012). These phenomena also interact with longer time-scale North Atlantic ocean-atmosphere phenomena such as the AMO (Section 14.7.6, Mariotti and Dell'Aquila, 2012; Sutton and Dong, 2012). Other phenomena have minor influence in limited sectors of the region (see Supplementary Material Section 14.SM.6.3).

Recent 1981-2012 trends in annual mean temperature in each subregion exceed the global mean land trend as can be inferred from Figure 2.22. Consistent with previous AR4 conclusions (Section 11.3), recent studies of extreme events (Section 2.6.1) point to a very likely increase of the number of warm days and nights, and decrease of the number of cold days and nights, since 1950 in Europe. Heat waves can be amplified by drier soil conditions resulting from warming (Vautard et al., 2007; Seneviratne et al., 2010; Hirschi et al., 2011). Several studies (Section 2.6.2.1) also indicate general increases in the intensity and frequency of extreme precipitation especially in winter during the last four decades however there are inconsistencies between studies, regions and seasons.

The ability of climate models to simulate the climate in this region has improved in many important aspects since AR4 (see Figure 9.38). Particularly relevant for this region are increased model resolution and a better representation of the land surface processes in many of the models that participated in the recent CMIP5 experiment. Table 14.2 provides an assessment of the CMIP5 quality for simulations of temperature, precipitation, and main phenomena in the region. The CMIP5 projections reveal warming in all seasons for the three sub-regions, while precipitation projections are more variable across sub-regions and seasons. In the winter half year (October to March), NEU and CEU are projected to have increased mean precipitation associated with increased atmospheric moisture, increased moisture convergence and 
intensification in ETC activity (Section 14.6.2 and Table 14.3) and no change or a moderate reduction in the MED. In the summer half year (April to September) , NEU and CEU mean precipitation are projected to have only small changes whereas there is a notable reduction in MED (see Table 14.1, Figures Al.36 to Al.37 and Al.42-Al.43). Figure 14.22 illustrates that the precipitation changes are broadly consistent with the findings CMIP3.

High-resolution projections from the Japanese high-resolution model ensemble also agree with these findings and are consistent with downscaling results from coordinated multi-model GCM/RCM experiments (e.g., ENSEMBLES, Déqué et al., 2012). In general, regional climate change amplitudes for temperature and precipitation follow the global warming amplitude although modulated both by changes in the large-scale circulation and by regional feedback processes (Kjellstrom et al., 2011), which confirms assessments in AR4 (Christensen et al., 2007).

Some new investigations have focussed on the uncertainties associated with model projections. A large ensemble of RCM-GCM shows that the temperature response is robust in spite of a considerable uncertainty related to choice of model combination (GCM/RCM) and sam- pling (natural variability), even for the 2021-2050 time frame (Déqué et al., 2012). Other studies based on CMIP3 projections suggest that GHG-forced changes in the MED are likely to become distinguishable from the 'noise' created by internal decadal variations in decades beyond 2020-2030 (Giorgi and Bi, 2009). It has been also shown using an ensemble of RCM simulations that the removal of NAO-related variability leads to an earlier emergence of change in seasonal mean temperatures for some regions in Europe (Kjellström et al., 2013). Hence, in the near term, decadal predictability is likely to be critically dependent on the regional impacts of modes of variability 'internal' to the climate system (Section 11.3). However, it has been shown that NAO trends do not account for a large fraction of the long-term future change in mean temperature or precipitation (Stephenson et al., 2006) and that largescale atmospheric circulation changes in CMIP5 models are not the main driver of the warming projected in Europe by the end of the century (2081-2100; Cattiaux et al., 2013). Therefore, changes in climate phenomena contribute to the uncertainty in the near-term projections rather than long-term changes in this region (Table 14.3), further supporting the credibility in model projection (Table 14.2).

Recent studies have clearly identified a possible amplification of temperature extremes by changes in soil moisture (Jaeger and Seneviratne,

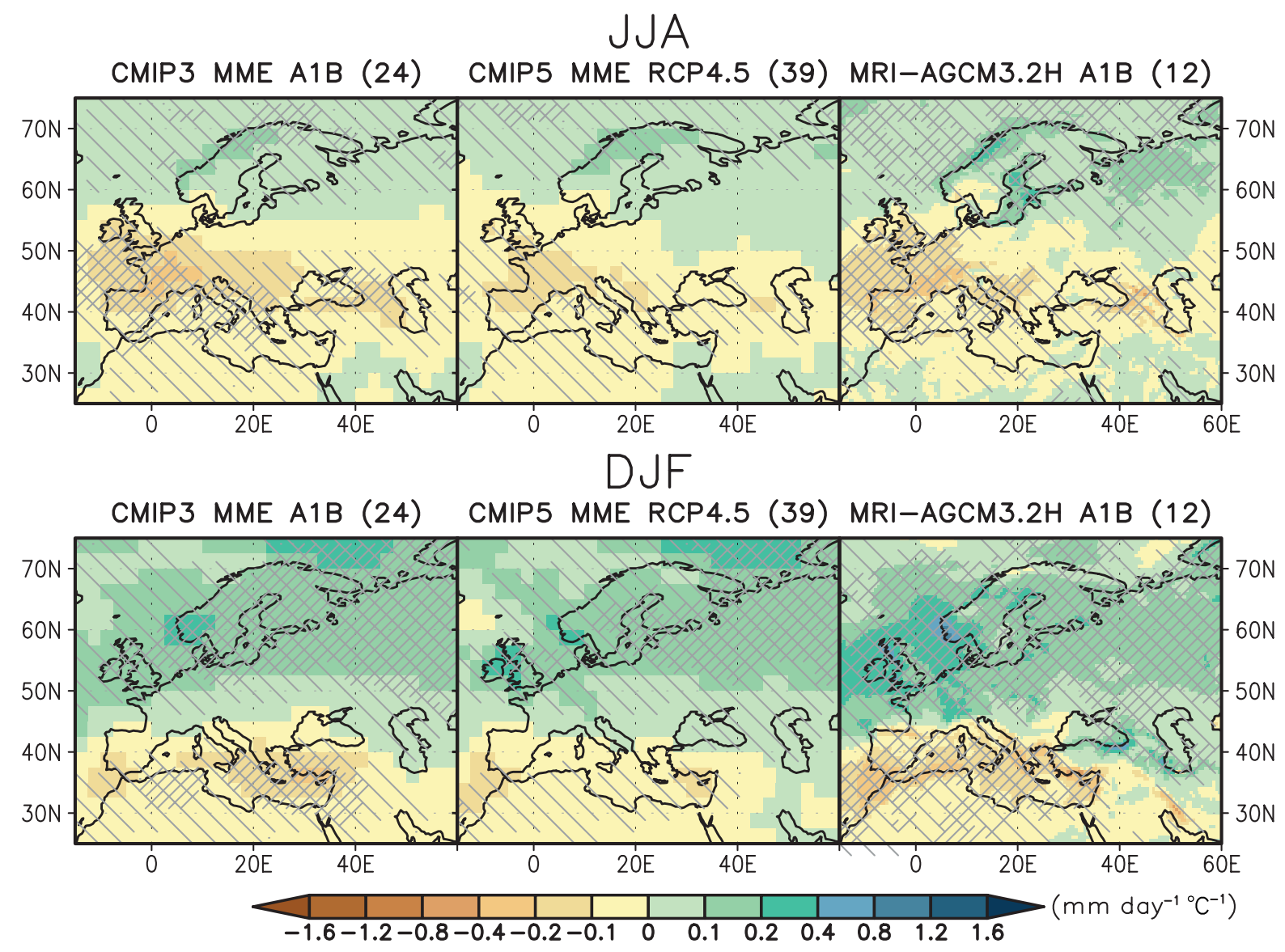

Figure 14.22 | Maps of precipitation changes for Europe and Mediterranean in 2080-2099 with respect to 1986-2005 in June to August (above) and December to February (below) in the SRES A1B scenario with 24 CMIP3 models (left), and in the RCP4.5 scenario with 39 CMIP5 models (middle). Right figures are the precipitation changes in 2075-2099 with respect to 1979-2003 in the SRES A1B scenario with the 12 member 60 km mesh Meteorological Research Institute (MRI)-Atmospheric General Circulation Model 3.2 (AGCM3.2) multi-physics, multi-sea surface temperature (SST) ensembles (Endo et al., 2012). Precipitation changes are normalized by the global annual mean surface air temperature changes in each scenario. Light hatching denotes where more than $66 \%$ of models (or members) have the same sign with the ensemble mean changes, while dense hatching denotes where more than $90 \%$ of models (or members) have the same sign with the ensemble mean changes. 
2010; Hirschi et al., 2011), acting as a mechanism that further magnifies the intensity and frequency of heat waves given the projected enhance of summer drying conditions. This is in line with the assessed results presented in SREX (Seneviratne et al., 2012). At the other end of the spectrum, studies indicate that European winter variability may be related to sea ice reductions in the Barents-Kara Sea (Petoukhov and Semenov, 2010) and CMIP5 models in projections for the future in general exhibit a similar relation until the summer sea ice has almost disappeared (Yang and Christensen, 2012). Although the mechanism behind this relation remains unclear this suggests that cold winters in Europe will continue to occur in coming decades, despite an overall warming.

Although climate models have improved fidelity in simulating aspects of regional climates over Europe and the Mediterranean, the spread in projections is still substantial, partly due to large amounts of natural variability in this region (particularly NAO and AMO), besides the inherent model deficiencies .

In summary, there is high confidence in model projections of mean temperature in this region. It is very likely that temperatures will continue to increase throughout the 21st century over all of Europe and the Mediterranean region. It is likely that winter mean temperature will rise more in NEU than in CEU or MED, whereas summer warming will likely be more intense in MED and CEU than in NEU. The length, frequency, and/or intensity of warm spells or heat waves are assessed to be very likely to increase throughout the whole region. There is medium confidence in an annual mean precipitation increase in NEU and CEU, while a decrease is likely in MED summer mean precipitation.

\subsubsection{Africa}

The African continent encompasses a variety of climatic zones. Here the continent is divided into four major sub-regions: Sahara (SAH), Western Africa (WAF), Eastern Africa (EAF) and Southern Africa (SAF). A fifth Mediterranean region to the north of Sahara is discussed in Section 14.8.6. In tropical latitudes, rainfall follows insolation (this simplified picture is modified by the presence of orography, especially in the Great Horn of Africa, the geography of the coastline, and by the oceans). The most relevant phenomena affecting climate variability are the monsoons (Section 14.2.4), ENSO (Section 14.4), Indian and Atlantic Ocean SSTs (IOD, Section 14.3.3; AMM Section 14.3.4; AMO Section 14.7.6) and the atmospheric Walker Circulation (Section 2.7.5). Tropical cyclones impact East African and Madagascan coastal regions (Section 14.6.1) and ETCs clearly impact southern Africa (Section 14.6.2).

Sub-Saharan Sahelian climate is dominated by the monsoonal system that brings rainfall to the region during only one season (Polcher et al., 2011). Most of the rain between May/June and September comes from mesoscale 'squall line' systems that travel short distances in their lifetime ( 1000 to $2000 \mathrm{~km}$ ), and whose distribution is somewhat modified by the synoptic scale African Easterly Wave (Ruti and Dell'Aquila, 2010). The onset of the rainy season in West Africa is a key parameter triggering changes in the vegetation and surface properties, that implies feedbacks to the local atmospheric heat and moisture cycle. The length and frequency of dry spells as well as the length or cumulated rainfall of the season also affect this. All are affected by a large interannual variability (Janicot et al., 2011). When evaluating models their ability to reproduce such characteristics of the African monsoon is essential. A large effect of natural multi-decadal SST and warming of the oceans on Sahel rainfall is very likely (Hoerling et al., 2006; Ting et al., 2009, 2011; Mohino et al., 2011; Rodriguez-Fonseca et al., 2011).

East Africa experiences a semi-annual rainfall cycle, driven by the ITCZ movement across the equator. Direct links between the region's rainfall and ENSO have been demonstrated (Giannini et al., 2008) and references therein), but variations in Indian Ocean SST (phases of the IOD) are recognized as the dominant driver of east African rainfall variability (Marchant et al., 2007). This feature acts to enhance rainfall through either anomalous low-level easterly flow of moist air into the continent (Shongwe et al., 2011), or a weakening of the low-level westerly flow over the northern Indian Ocean that transports moisture away from the continent (Black et al., 2003). Although the effect of the IOD is evident in the short rainy season, Shongwe et al. (2011) do not find a similar relationship for the long rains. Williams and Funk (2011), however, argue for a reduction in the long rains over Kenya and Ethiopia in response to warmer Indian Ocean SSTs.

Variability in southern Africa's climate is strongly influenced by its adjacent oceans (Rouault et al., 2003; Hansingo and Reason, 2008, 2009; Hermes and Reason, 2009) as well as by ENSO (Vigaud et al., 2009; Pohl et al., 2010). Although it is generally observed that El Niño events correspond to conditions of below-average rainfall over much of southern Africa (Mason, 2001; Giannini et al., 2008; Manatsa et al., 2008) the ENSO teleconnection is not linear, but rather has complex influence in which a number of regimes of local rainfall response can be identified (Fauchereau et al., 2009). The extreme southwestern parts of southern Africa receive rainfall in austral winter brought by mid-latitude frontal systems mostly associated with passing ETCS, but the majority of the region experiences a single summer rainfall season occurring between November and April. A semi-permanent zone of sub-tropical convergence is a major contributor to summer rainfall in sub-tropical southern Africa (Fauchereau et al., 2009; Vigaud et al., 2012).

Because of its exceptional magnitude and its clear link to global SST, 20th century decadal rainfall variability in the Sahel is a test of GCMs ability to produce realistic long-term changes in tropical precipitation. Despite biases in the region (Cook and Vizy, 2006) the CMIP3 coupled models overall can capture the observed correlation between Sahel rainfall and basin-wide area averaged SST variability (Biasutti et al., 2008) even though individual models may fail, especially at interannual time scales (Lau et al., 2006; Joly et al., 2007). Recently, Ackerley et al. (2011) used a perturbed physics ensemble and reached a similar result for the role of atmospheric sulphate, confirming previous results (Rotstayn and Lohmann, 2002; Held et al., 2005). Since AR4, only limited information about improved performance has been documented and only in WAF have major efforts been focussing on relating model behaviour with ability to simulate local climate processes in such details. However, in a comparative study of the ability of CMIP3 and CMIP5 to simulate multiple SST-Africa teleconnections, Rowell (2013) found varying degrees of success in simulating these. In particular, no clear indication of an improvement in the CMIP5 models vs. the CMI3 models was identified. 
In projections of the 21st century, the CMIP3 models produced both significant drying and significant moistening (Held et al., 2005; Biasutti and Giannini, 2006; Cook and Vizy, 2006; Lau et al., 2006), and the mechanisms by which a model dries or wets the Sahel are not fully understood (Cook, 2008). At least qualitatively, the CMIP3 ensemble simulates a more robust response during the pre-onset and the demise portion of the rainy season (Biasutti and Sobel, 2009; Seth et al., 2011). Rainfall is projected to decrease in the early phase of the seasons-implying a small delay in the main rainy season, but is projected to increase at the end of the season-implying an intensification of late-season rains (d'Orgeval et al., 2006), although this appears to be less robust in the CMIP5 models (Section 14.2.4). Projections of a change in the timing of the rains is common to other monsoon regions (Li et al., 2006; Biasutti and Sobel, 2009; Seth et al., 2011), including southern Africa.
The relevance of a local effect is supported by several lines of evidence. There is observational evidence that local soil moisture gradients can trigger convective systems and that these surface contrasts are as important as topography for generating these systems, which bring most of the rain to the region (Taylor et al., 2011a, 2011b). Additional evidence comes from simulations of future rainfall changes in West Africa by RCMs subject to coupled model-derived boundary conditions (Patricola and Cook, 2010), documenting a wetting response of the Sahel to increased GHG in the absence of other forcings. But the relative importance of this effect versus the response to SST trends is not well quantified, mostly due to the limitation of using a single RCM.

An evaluation of six GCMs over East Africa by Conway et al. (2007) reveals no clear multi-model trend in mean annual rainfall by the 2080s, but some indications of increased SON and decreased March,

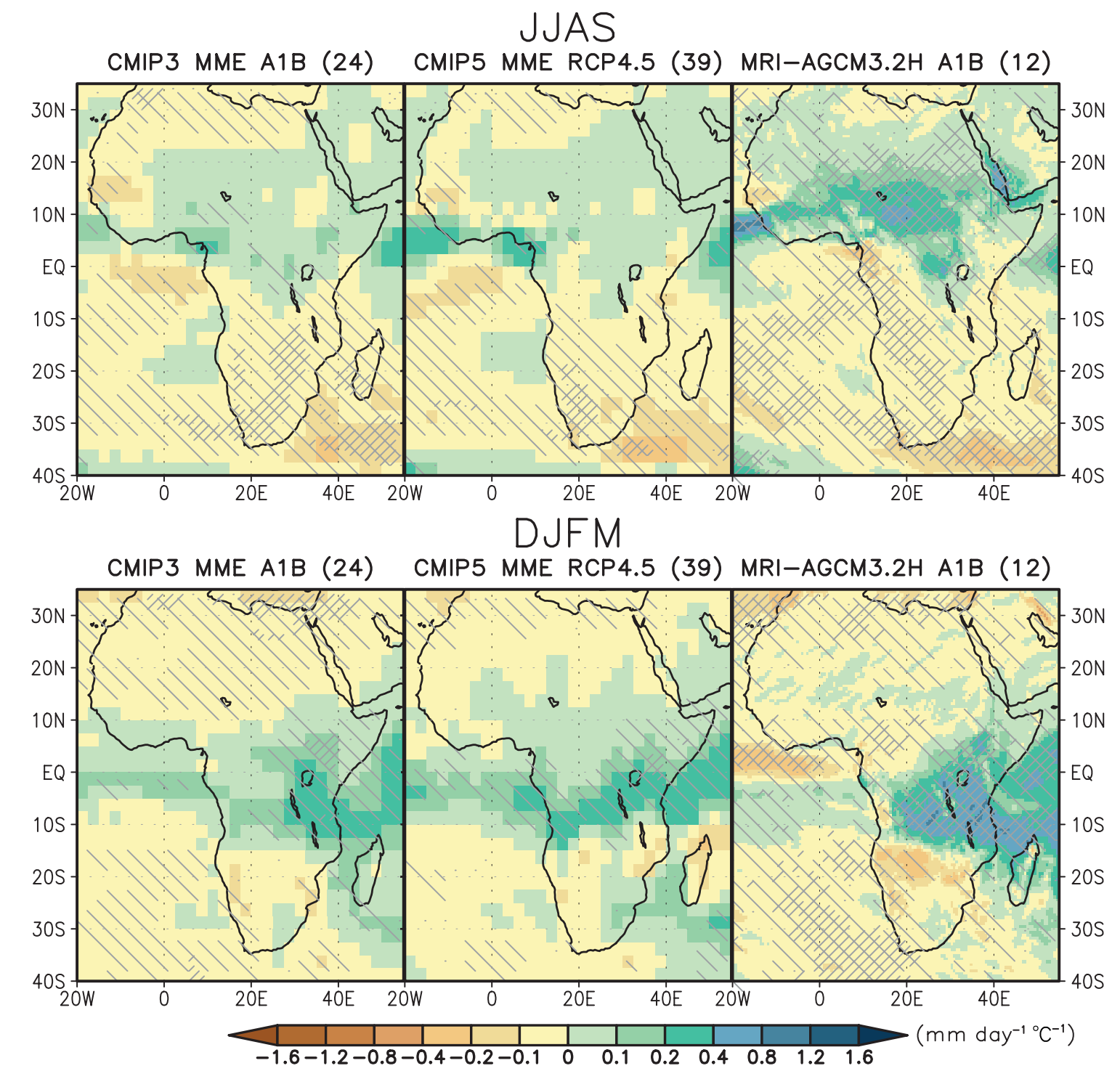

Figure 14.23 | Maps of precipitation changes for Africa in 2080-2099 with respect to 1986-2005 in June to September (above) and December to March (below) in the SRES A1B scenario with 24 CMIP3 models (left), and in the RCP4.5 scenario with 39 CMIP5 models (middle). Right figures are the precipitation changes in 2075-2099 with respect to 1979-2003 in the SRES A1B scenario with the 12-member 60-km mesh Meteorological Research Institute (MRI)-Atmospheric Generl Circulation Model 3.2 (AGCM3.2) multiphysics, multi-sea surface temperature (SST) ensembles (Endo et al., 2012). Precipitation changes are normalized by the global annual mean surface air temperature changes in each scenario. Light hatching denotes where more than $66 \%$ of models (or members) have the same sign with the ensemble mean changes, while dense hatching denotes where more than $90 \%$ of models (or members) have the same sign with the ensemble mean changes. 
April and May (MAM) rainfall are noted. They found inconsistency in how the models represent changes in the IOB and consequent changes in rainfall over East Africa. Shongwe et al. (2011) analysed an ensemble of 12 CMIP3 GCMs (forced with A1B emissions). They found widespread increases in short season (OND) rainfall including extreme precipitation across the region, with statistically significant ensemble mean increases. For the long rains (MAM), similar changes in the sign and magnitude of mean and extreme seasonal rainfall were seen, but model skill in simulating the MAM season is relatively poor. The changes shown for the short rains are consistent with a differential warming of 21 st century Indian Ocean SSTs, which leads to a positive IOD-like state (see Section 14.3.3). The atmospheric consequence of this is a weakening of the descending branch of the East African Walker Cell and enhancement of low-level moisture convergence over east Africa (Vecchi and Soden, 2007a; Shongwe et al., 2011).

In an assessment of 19 CMIP3 models run with the A1B emissions forcing, Giannini et al. (2008) note a tendency toward a persistent El Niño-like pattern (see Section 14.4) in the equatorial Pacific along with a decrease in rainfall over southern Africa. Dynamical downscaling of a single GCM by Engelbrecht et al. (2011) shows - for the austral winter-an intensification of the southern edge of the subtropical high pressure belt resulting in southward displacement of the mid-latitude systems that bring frontal rain to the south western parts of the continent, thus resulting in decrease in rainfall. The decrease in summer rainfall is consistent with high-resolution $(18 \mathrm{~km}) \mathrm{RCM}$ simulations done by Haensler et al. (2011) which indicate widespread reductions in rainfall over southern Africa under the A1B scenario.

Shongwe et al. (2009) identified reduction in spring (SON) rainfall throughout the eastern parts of southern Africa. There is good consensus amongst the models used, with the spring anomalies indicating a trend toward later onset of the summer rainy season. Autumn (MAM) reductions are shown for most of southern Africa while eastern South Africa experiences no change and eastern parts of southern Africa show a small increase.

Table 14.2 provides an overall assessment of CMIP5 quality for simulations of temperature, precipitation, and main phenomena in the different sub-regions of Africa. Overall, confidence in the projected precipitation changes is at best medium. This is owing to the overall modest ability of models to capture the most important phenomena having a strong control on African climates (Table 14.3).

The ability of climate models to simulate historical climate, its change, and its variability, has improved in many aspects since the AR4 (see Section 9.6.1). But for Africa there is no clear evidence that the modest increase in resolution and a better representation of the land surface processes in many CMIP5 models have resulted in marked improvements (e.g., Figure 9.39). The CMIP5 models projection for this century is further warming in all seasons in the considered four sub-regions, while precipitation show some distinct sub-regional and seasonally dependent changes. In the October to March half year all four regions are projected to receive practically unaltered precipitation amounts by 2081-2100, although somewhat elevated in RCP8.5. In the April to September half year SAH, WAF and EAF will experience little change but a quite notable reduction in SAF is projected (see Table 14.1,
Figures Al.40 to Al.51). This is consistent with the results from CMIP3 as depicted in Figure 14.23 in the West African monsoon wet season and austral summer. High resolution information provided by the Japanese high-resolution model ensemble also matches this finding.

In summary, given models' ability to capture local processes, large scale climate evolution and their linkages, it is very likely that all of Africa will continue to warm during the 21 st century. The overall quality of the CMIP5 models imply, that SAH already very dry is very likely to remain very dry. But there is low confidence in projection statements about drying or wetting of WAF. Owing to models' ability to capture the overall monsoonal behaviour, there is medium confidence in projections of a small delay in the rainy season with an increase at the end of the season. There is medium confidence in projections showing little change in mean precipitation in EAF and reduced precipitation in the Austral winter in SAF, as models tend to represent Indian Ocean SST developments with credibility. Likewise, increasing rainfall in EAF is likely for the short rainy season, but low confidence exists in projections regarding drying or wetting in the long rainy season.

\subsubsection{Central and North Asia}

This area mostly covering the interior of a large continent extending from the Tibetan plateau to the Arctic is mainly influenced by weather systems coming from the west or south, giving some dependency on the AAM (Section 14.2.2) on the one hand and NAO/NAM (Section 14.5.1) on the other, with associated atmospheric blocking as an additional phenomenon of influence related to the latter (Box 14.2). In particular, the variability and long-term change of the climate system in central Asia and northern Asia are closely related to variations of the NAO and NAM (Takaya and Nakamura, 2005; Knutson et al., 2006; Popova and Shmakin, 2010; Sung et al., 2010; Table 14.3).

As a part of the polar amplification, large warming trends in recent decades are observed in the northern Asian sector (e.g., Figure 2.22). The warming trend was particularly strong in the cold season (November to March), with an increase of $2.4^{\circ} \mathrm{C}$ per 50 years in the mid-latitude semi-arid area of Asia, where the annual rainfall is within the range of 200 to $600 \mathrm{~mm}$ over the period of 1901-2009 (Huang et al., 2012). The observations indicate some increasing trends of heavy precipitation events in northern Asia, but no spatially coherent trends in central Asia (Seneviratne et al., 2012).

The CMIP5 models generally have difficulties in representing the mean climate expressed as the climatological means of both temperature and precipitation (Table 14.2) for the sub-regions represented in this area, which is partly related to the poor resolution unable to resolve the complex mountainous terrain dominating this region. But the scarceness of observational data and issues related to how these best can be compared with coarse resolution models adds to the uncertainty regarding model quality.

The model projections presented in AR4 (Section 11.4) indicated strong warming in northern Asia during winter and in central Asia during summer. Precipitation was projected to increase throughout the year in northern Asia with the largest fractional increase during winter. For central Asia, a majority of the CMIP3 models projected decreasing 

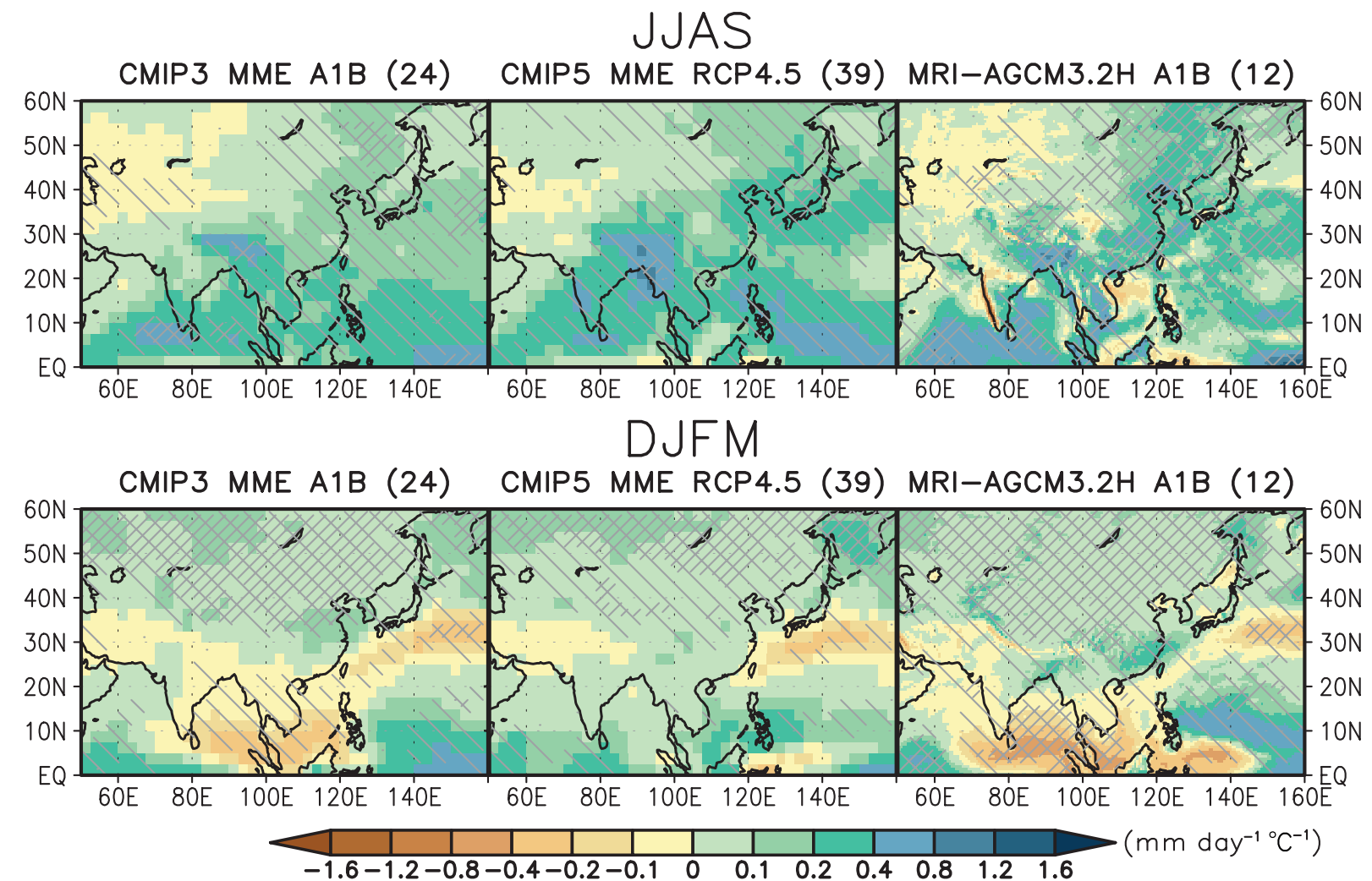

Figure 14.24 | Maps of precipitation changes for Central, North, East and South Asia in 2080-2099 with respect to 1986-2005 in June to September (above) and December to March (below) in the SRES A1B scenario with 24 CMIP3 models (left), and in the RCP4.5 scenario with 39 CMIP5 models (middle). Right figures are the precipitation changes in 2075-2099 with respect to 1979-2003 in the SRES A1B scenario with the 12-member 60-km mesh Meteorological Research Institute (MRI)-Atmospheric General Circulation Model 3.2 (AGCM3.2) multi-physics, multi-sea surface temperature (SST) ensembles (Endo et al., 2012). Precipitation changes are normalized by the global annual mean surface air temperature changes in each scenario. Light hatching denotes where more than $66 \%$ of models (or members) have the same sign with the ensemble mean changes, while dense hatching denotes where more than $90 \%$ of models (or members) have the same sign with the ensemble mean changes.

precipitation during spring and summer. Seneviratne et al. (2012) indicate increases in all precipitation extreme indices for northern Asia and in the 20-year return value of annual maximum daily precipitation for central Asia. These projections are supported by output from CMIP5 models subject to various RCP scenarios (see Annex I). CMIP5 projected temperature increase in Central Asia of comparable magnitude in both JJA and in DJF. In North Asia, temperatures rise more in DJF than in JJA, while less annual variation is found over Central Asia and the Tibetan Plateau (Table 14.1, Figures Al.12 to Al.13, Al.52 to Al.55 and Al.56 to Al.57).

With an RCM Sato et al. (2007) projected precipitation decreases over northern Mongolia and increases over southern Mongolia in July. Soil moisture over Mongolia decreases in July as a result of the combined effect of decreased precipitation and increased potential evaporation due to rising surface temperature. In North Asia, all CMIP5 models projects an increase in precipitation in the winter half year, and summer half year precipitation is also projected to increase (Table 14.1; Figures Al.14 to Al.15). In Central Asia and the Tibetan Plateau, model agreement is lower on changes both for winter and summer precipitation (Figure 14.24; Table 14.1; Figures Al.54 to Al.55 and Al.58 to Al.59). The ability of these CMIP5 models to simulate precipitation over this region varies (Table 14.3). The reasonable level of agreement in projections of precipitation to be positive and significantly above the 20 -year natural variability (Table 14.2), and therefore suggests that confidence in the sign of the projected change in future precipitation is medium.

In summary, all the areas are projected to warm, a stronger than global mean warming trend is projected for northern Asia during winter. For central Asia, warming magnitude is similar between winter and summer. Precipitation in northern Asia will very likely increase, whereas the precipitation over central Asia is likely to increase. Extreme precipitation events will likely increase in both regions.

\subsubsection{East Asia}

Summer is the rainy season for East Asia. The Meiyu-Changma-Baiu rain band is the defining feature of East Asian summer climate, extending from eastern China through central Japan (Ding and Chan, 2005; Zhou et al., 2009b). The summer rain band is anchored by the subtropical westerly jet (Sampe and Xie, 2010), and located on the northwestern flank of the western North Pacific subtropical high (Zhou and $\mathrm{Yu}, 2005)$. The wintertime circulation is characterized by monsoonal northerlies between the Siberian High and the Aleutian Low.

Both the East Asian summer and winter monsoon circulations have experienced an inter-decadal scale weakening after the 1970s due to natural variability of the coupled climate system, leading to enhanced 
(a) PRCTOC

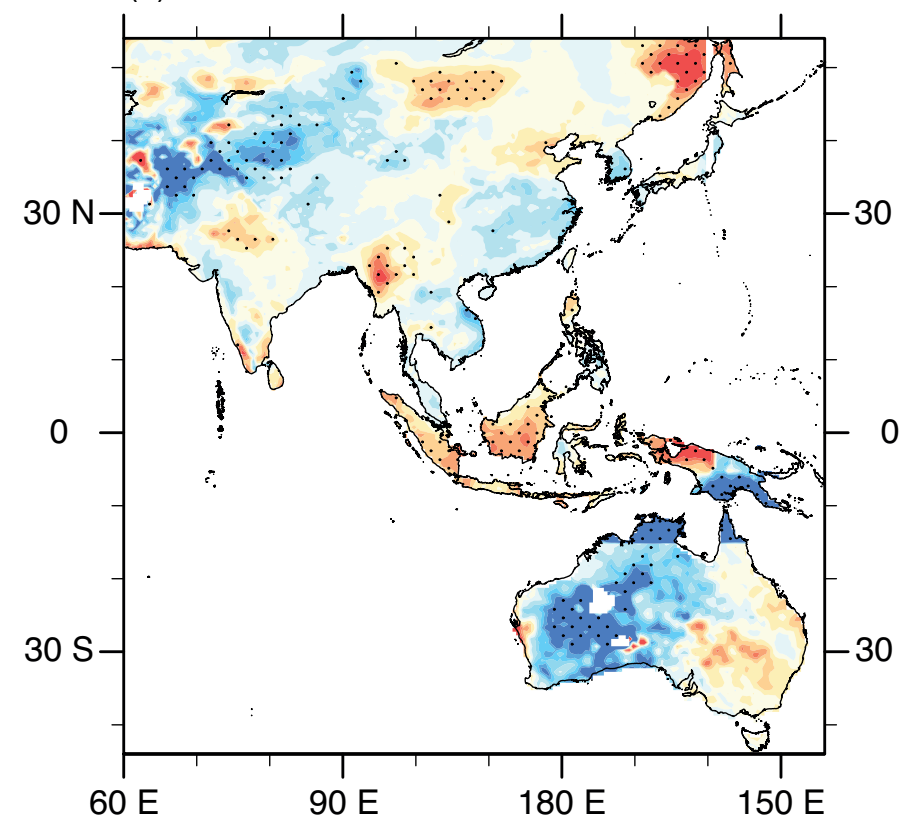

(c) R95

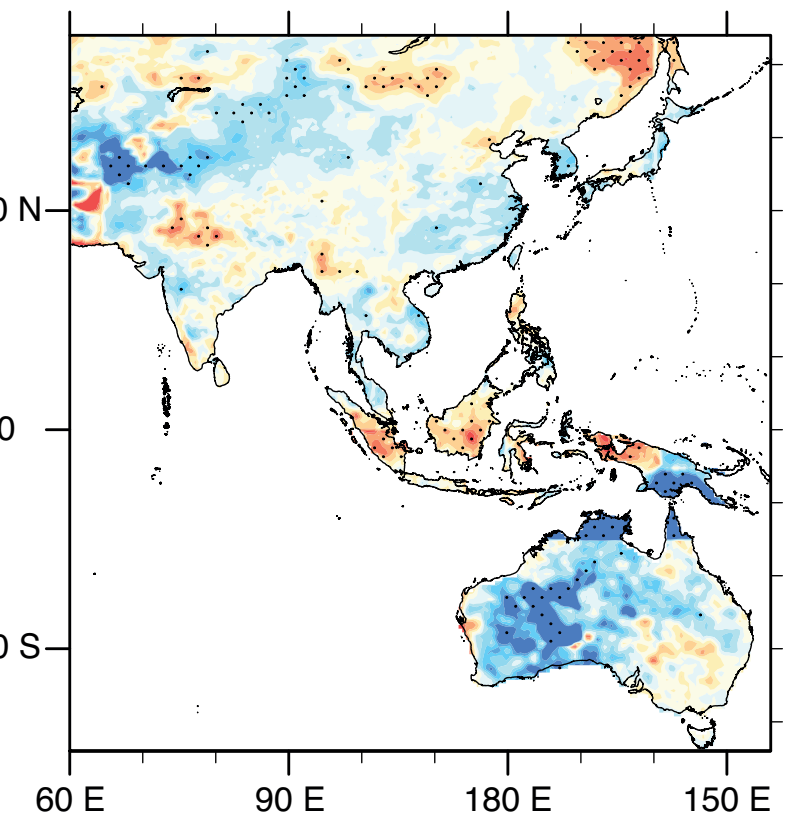

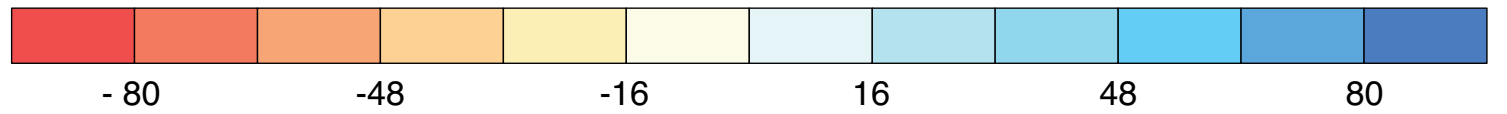

(\% per $50 \mathrm{yr})$

Figure 14.25 | Linear trend for local summer (a) total precipitation and (b) R95 (summer total precipitation when PR >95th percentile) during 1961-2006. The unit is \% per 50 -years. The trends statistically significant at the $5 \%$ level are dotted. The daily precipitation data over Australia and China are produced by the Australian Water Availability Project (AWAP, Jones et al., 2009a) and National Climate Centre China of China Meteorological Administration (Wu and Gao, 2013), respectively, while that over the other area is compiled by the Highly Resolved Observational Data Integration Towards the Evaluation of Water Resources (APHRODITE) project (Yatagai et al., 2012). The resolution of precipitation data set is $0.5^{\circ} \times 0.5^{\circ}$. Local summer is defined as June, July and August in the Northern Hemisphere, and December, January and February in the Southern Hemisphere.

mean and extreme precipitation along the Yangtze River Valley $\left(30^{\circ} \mathrm{N}\right)$ but deficient mean precipitation in North China in summer (Figure 14.25), and a warmer climate in winter. The observed monsoon circulation changes are partly reproduced by GCMs driven by PDO-related SST patterns but the quality of precipitation simulation is poor (Zhou et al., 2008a; Li et al., 2010a; Zhou and Zou, 2010).

In AR4, the regional warming is projected to be above the global mean in East Asia (Christensen et al., 2007). "It is very likely that heat waves/hot spells in summer will be of longer duration, more intense and more frequent, but very cold days are very likely to decrease in frequency. The precipitation is likely to increase in both boreal winter and summer, while the frequency of intense precipitation events is very likely to increase. Extreme rainfall and winds associated with tropical cyclones are likely to increase". CMIP5 results support many of these assessments.

More recent analysis suggested that CMIP3 models projected increased summer precipitation in amount and intensity over East Asia (Figure 14.24 for SRES A1B scenario) due to enhanced moisture convergence in a warmer climate (Ding et al., 2007; Sun and Ding, 2010; Chen et al., 2011; Kusunoki and Arakawa, 2012), along with an increase in interannual variability (Lu and Fu, 2010). CMIP5 projections for RCP4.5 support those from AR4 for summer (Figure 14.24), with $90 \%$ of the models projecting a precipitation increase in the winter half year (see Table 14.1 and Figures Al.56 to Al.59). CMIP3 models projections indicated a decrease of winter precipitation extending northeastward from South China Sea to south of Japan under SRES A1B scenario, changes seen in CMIP5 projections but with smaller spatial coverage (Figure 14.24).

An increase of extreme precipitation is projected over East Asia in a warmer climate (Jiang et al., 2011; Lee et al., 2011; Li et al., 2011a, $2011 \mathrm{~b})$. A high-resolution model projects an increase of Meiyu precipitation in May through July, Changma precipitation over Korean peninsula in May, and Baiu precipitation over Japan in July (Kusunoki and Mizuta, 2008), and an increase of heavy precipitation over East Asia under SRES A1B scenario (Kusunoki and Mizuta, 2008; Endo, 2012). CMIP3 models project a late withdrawal of Baiu (Kitoh and Uchiyama, 2006), as has been observed in eastern and western Japan (Endo, 2010). There is a significant increase in mean, daily maximum and minimum temperatures in southeastern China, associated with a decrease in the number of frost days and an increase in the heat wave duration under SRES A2 scenario (Chen et al., 2011). The CMIP5 model projections also indicate an increase of temperature in both boreal winter and summer over East Asia for RCP4.5 (Table 14.1). A decrease of the annual and seasonal maximum wind speeds is found under SRES A2 scenario due to both the reduced intensity of cold waves and the reduced intensity of the winter monsoons (Jiang and Zhao, 2013). 
The future warming patterns simulated by RCMs essentially follow those of the driving GCMs (e.g., Dairaku et al., 2008). For summer precipitation, however, RCM downscaling usually shows different regional details due to more realistic topographic forcing than in GCMs (Gao et al., 2008, 2012a). The uncertainty of precipitation projection in eastern China is larger than that in western China (Gao et al., 2012b). RCM downscaling indicates that both the seasonal mean summer rainfall and extreme precipitation around Japan Islands are projected to increase (Im et al., 2008; lizumi et al., 2012).

Projections with a 5-km RCM show that the heaviest hourly precipitation is projected to increase even in the near future (2030s) when temperature increase is modest (Kitoh et al., 2009). A southwest expansion of the subtropical anticyclone over the northwestern Pacific Ocean associated with El Niño-like mean state changes in the Pacific and a dry air intrusion in the mid-troposphere from the Asian continent gives a favourable condition for intense precipitation in the Baiu season in Japan (Kanada et al., 2010). Increased water vapour supply from the south of the Baiu front and an intensified frontal zone with intense mean updrafts contribute to the increased occurrence of intense daily precipitation during the late Baiu season (Kanada et al., 2012).

In summary, based on CMIP5 model projections, there is medium confidence that with an intensified East Asian summer monsoon, summer precipitation over East Asia will increase (Table 14.3). Under RCP4.5 scenario, precipitation increase is likely over East Asia during the Meiyu-Changma-Baiu season in May to July, and precipitation extremes are very likely to increase over the eastern Asian continent in all seasons and over Japan in summer. However, there is only low confidence in more specific details of the projected changes due to the limited skill of CMIP5 models in simulating monsoon features such as the East Asian monsoon rainband (Table 14.2).

\subsubsection{West Asia}

This region extends from the Mediterranean to the western fringes of South Asia, covering the Middle East and the Arabian Peninsula and includes large areas of barren desert. The climate over this region varies from arid to semi-arid and precipitation is primarily received in the cold season.

The western part of the region is on the margin of Atlantic and Mediterranean influences, primarily the NAO (Section 14.5.1) during winter months, and indirectly the monsoon heat low (Section 14.2.2.1) in the summer months. Precipitation in this region comes largely from passing ETCs (Section 14.6.2). Land-falling TCs (Section 14.6.1) that occasionally influence the eastern part of the Arabian Peninsula are notable extreme events. Pacific Ocean variability, associated with ENSO (Section 14.2.4), and the ITCZ (Section 14.3.1) are also known to impact weather and climate in different parts of West Asia.

In recent decades, there appears to be a weak but non-significant downward trend in mean precipitation (Zhang et al., 2005; Alpert et al., 2008; AlSarmi and Washington, 2011; Tanarhte et al., 2012), although intense weather events appear to be increasing (Alpert et al., 2002; Yosef et al., 2009). In contrast, upward temperature trends are notable and robust (Alpert et al., 2008; AlSarmi and Washington, 2011; Tanarhte et al., 2012).

The ability of climate models to simulate historical climate, its change and its variability, has improved in many important aspects since the AR4 (see Figure 9.39 in Chapter 9). CMIP5 models tend to be able to reproduce the basic climate state of the region as well as the main phenomena affecting it with some fidelity (Table 14.2), but the region is at the fringes of the influence of different drivers of European, Asian and African climates and remains poorly analysed in the peer-reviewed literature with respect to climate model performances.

The CMIP5 model projections for this century are for further warming in all seasons, while precipitation shows some distinct sub-regional and seasonally dependent changes, characterized by model scatter. In both winter (October to March) and summer (April to September) precipitation in general is projected to decrease, (see Table 14.1, Figures Al.52 to Al.55). However, the various interacting dynamical influences on precipitation of the region (that models have varying success in capturing in the current climate) results in uncertainty in both the patterns and magnitude of future precipitation change. Indeed, while the overall pattern of change has remained the same between CMIP3 and CMIP5, the confidence has decreased somewhat and the boundary between the Mediterranean decreases and the general mid-latitude increase to the north has shifted closer to the region (Figures 14.26 and Al.54 to Al.55). So, although the Mediterranean side still appears likely to become drier, the likely precipitation changes for the interior land masses are less clear and the intensified and northward shifting ITCZ may imply an increase in precipitation in the most southern part of the Arabian Peninsula. Overall, the projections by the end of the century (2081-2100) indicates little overall change, although with a tendency for reduced precipitation, particular in the high end scenarios (Figures Al.5 to Al.55). However, regardless of the sign of precipitation change in the high mountain regions of the interior, the influence of warming on the snow pack will very likely cause important changes in the timing and amount of the spring melt (Diffenbaugh et al., 2013).

Recent downscaling results (Lionello et al., 2008; Evans, 2009; Jin et al., 2010; Dai, 2011) suggest that the eastern Mediterranean will experience a decrease in precipitation during the rainy season due to a northward displacement of the storm tracks (Section 14.6.2). A northward shift in the ITCZ results in more precipitation in the southern part, not previously being seriously affected by it. A moderate change in the annual cycle of precipitation has also been simulated by some models. Precipitation and temperature statistics in RCMs for an area consisting of the western part of the Arab Peninsula was assessed by Black (2009) and Onol and Semazzi (2009) confirming GCM-based findings. Increased drought duration has been projected (Kim and Byun, 2009). Inland from the Mediterranean coastal areas, resolution of the terrain becomes more important and, while downscaled results (Evans, 2008; Marcella and Eltahir, 2011; Lelieveld et al., 2012) broadly agree with GCM projections, higher resolution results in some differences associated with mountain barrier jets (Evans, 2008; see also Figure 14.26).

In summary, since AR4 climate models appear to have only modestly improved fidelity in simulating aspects of large-scale climate 


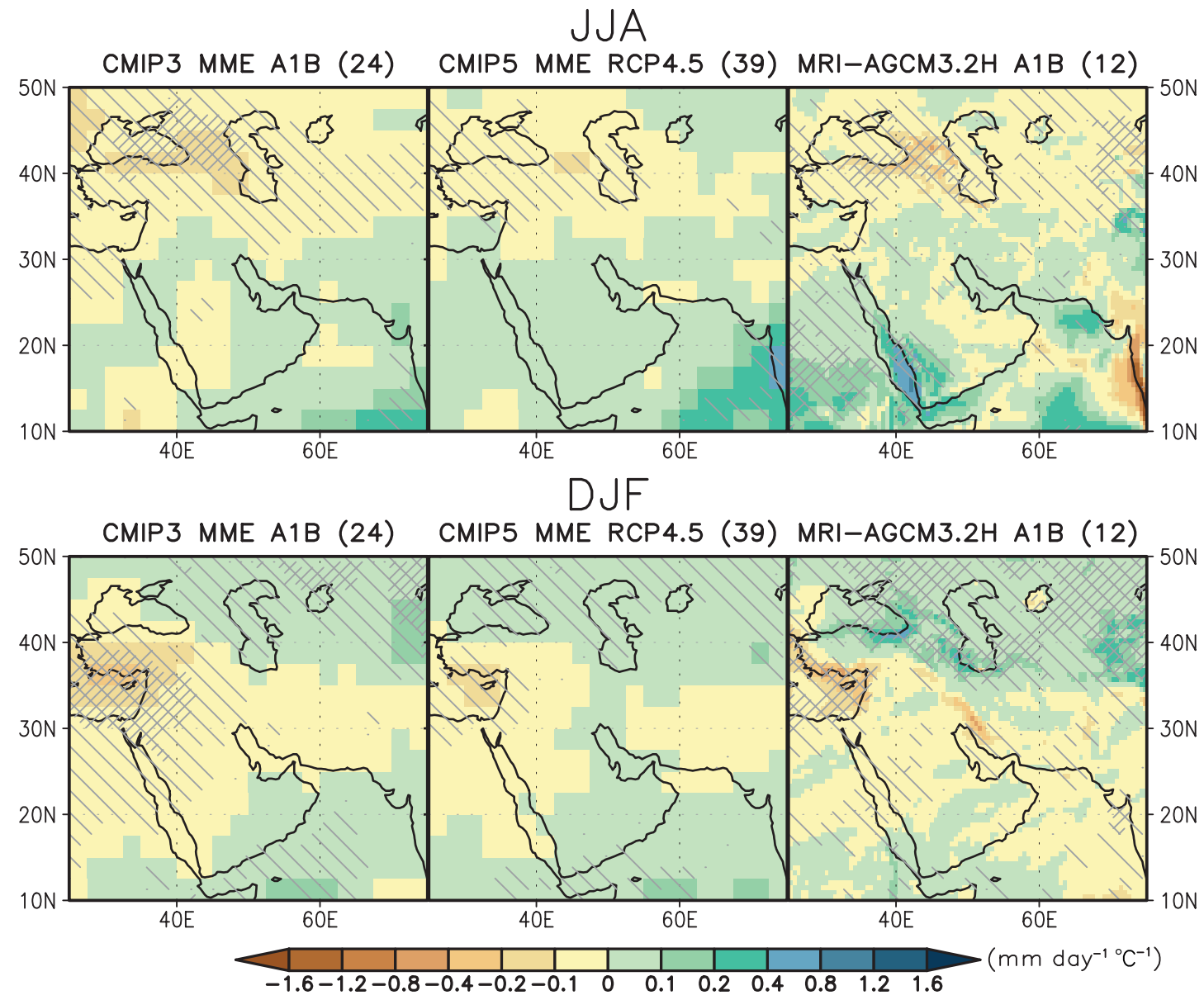

Figure 14.26 | Maps of precipitation changes for West Asia in 2080-2099 with respect to 1986-2005 in June, July and August (above) and December, January and February (below) in the SRES A1B scenario with 24 CMIP3 models (left), and in the RCP4.5 scenario with 39 CMIP5 models (middle). The figures on the right are the precipitation changes in 2075-2099 with respect to 1979-2003 in the SRES A1B scenario with the 12-member 60-km mesh Meteorological Research Institute (MRI)-Atmospheric General Circulation Model 3.2 (AGCM3.2) multi-physics, multi-sea surface temperature (SST) ensembles (Endo et al., 2012). Precipitation changes are normalized by the global annual mean surface air temperature changes in each scenario. Light hatching denotes where more than $66 \%$ of models (or members) have the same sign with the ensemble mean changes, while dense hatching denotes where more than $90 \%$ of models (or members) have the same sign with the ensemble mean changes.

phenomena influencing regional climates over West Asia. Model agreement, however, indicates that it is very likely that temperatures will continue to increase. But at the same time, model agreement on projected precipitation changes have reduced, resulting in medium confidence in projections showing an overall reduction in precipitation.

\subsubsection{South Asia}

From June through September, the Indian summer monsoon (Section 14.2.2.1) dominates South Asia, while the northeast winter monsoon contributes substantially to annual rainfall over southeastern India and Sri Lanka. The winter weather systems are also important in northern parts of South Asia, that is, the western Himalayas.

Seasonal mean rainfall shows interdecadal variability, noticeably a declining trend with more frequent deficit monsoons (Kulkarni, 2012). There are regional inhomogeneities: precipitation decreased over central India along the monsoon trough (Figure 14.25) thought to be due to a number of factors (Section 14.2.2) including black carbon, sulphate aerosols (Chung and Ramanathan, 2007; Bollasina et al., 2011), land use changes (Niyogi et al., 2010) and SST rise over the Indo-Pacific warm pool (Annamalai et al., 2013). The increase in the number of monsoon break days over India (Dash et al., 2009), and the decline in the number of monsoon depressions (Krishnamurthy and Ajayamohan, 2010), are consistent with the overall decrease in seasonal mean rainfall. The frequency of heavy precipitation events is increasing (Rajeevan et al., 2008; Krishnamurthy et al., 2009; Sen Roy, 2009; Pattanaik and Rajeevan, 2010), while light rain events are decreasing (Goswami et al., 2006).

CMIP models reasonably simulate the annual cycle of precipitation and temperature over South Asia (Table 14.2; Figure 9.38) but are limited in simulating fine structures of rainfall variability on sub-seasonal and sub-regional scales (Turner and Annamalai, 2012). CMIP5 models show improved skill in simulating monsoon variability compared to CMIP3 (Sperber et al., 2012; Section 14.2.2).

Summer precipitation changes in South Asia are consistent overall between CMIP3 and CMIP5 (Figure 14.24), but model scatter is large in winter precipitation change (Figures 14.24 and Al.62). Changes in the summer monsoon dominate annual rainfall (see Section 14.2.2). The CMIP3 multi-model ensemble shows an increase in summer 
precipitation (Kumar et al., 2011a; May, 2011; Sabade et al., 2011), although there are wide variations among model projections (Annamalai et al., 2007; Kripalani et al., 2007b). Spatially, the rainfall increase is stronger over northern parts of South Asia, Bangladesh and Sri Lanka, with a weak decrease over Pakistan (Turner and Annamalai, 2012). In RCP6.0 and RCP8.5 scenarios, frequency of extreme precipitation days shows consistent increasing trends in 2060 and beyond (Chaturvedi et al., 2012; Figure Al.63). In six CMIP3 models, precipitation anomalies during Indian summer monsoon breaks strengthen in a warmer climate, but changes in the timing and duration of active/break spells are variable among models (Mandke et al., 2007). Note that the active/break spells of the monsoon are related to the MJO (see Section 14.3.2), a phenomenon that models simulate poorly (Section 9.5.2.3; Lin et al., 2008a; Sperber and Annamalai, 2008).

High-resolution RCM and GCM projections showed an overall increase of precipitation over a large area of peninsular India (Rupa Kumar et al., 2006; Stowasser et al., 2009; Kumar et al., 2011a), but a significant reduction in orographic rainfall in both seasonal mean and extreme events on west coasts of India (Rajendran and Kitoh, 2008; Ashfaq et al., 2009; Kumar et al., 2013). Such spatial variations in projected precipitation near orography are noticeable in Figure 14.24 on the background of the overall increase.

CMIP5 models project a clear increase in temperature over India especially in winter (Figures Al.60 to Al.61), with enhanced warming during night than day (Kumar et al., 2011a) and over northern India (Kulkarni, 2012). In summer, extremely hot days and nights are projected to increase. Table 14.1 summarizes the projected temperature and precipitation changes for SAS in the RCP4.5 scenario based on CMIP5.

In summary, there is high confidence in projected rise in temperature. There is medium confidence in summer monsoon precipitation increase in the future over South Asia. Model projections diverge on smaller regional scales.

\subsubsection{Southeast Asia}

Southeast Asia features a complex range of terrains and land-sea contrasts. Across the region, temperature has been increasing at a rate of $0.14^{\circ} \mathrm{C}$ to $0.20^{\circ} \mathrm{C}$ per decade since the 1960 s (Tangang et al., 2007), coupled with a rising number of hot days and warm nights, and a decline in cooler weather (Manton et al., 2001; Caesar et al., 2011). A positive trend in the occurrence of heavy (top 10\% by rain amount) and light (bottom 5\%) rain events and a negative trend in moderate ( 25 to $75 \%$ ) rain events has been observed (Lau and Wu, 2007). Annual total wet-day rainfall has increased by $22 \mathrm{~mm}$ per decade, while rainfall from extreme rain days has increased by $10 \mathrm{~mm}$ per decade (Alexander et al., 2006; Caesar et al., 2011).

Several large-scale phenomena influence the climate of this region. While ENSO (Section 14.4) influence is predominant in East Malaysia and areas east of it, Maritime continent monsoon (Section 14.2.3) influences the climate in Peninsular Malaya. The impact of the IOD (Section 14.3.3) is more prominent in eastern Indonesia. Thus climate variability and trends differ vastly across the region and between seasons. Between 1955 and 2005 the ratio of rainfall in the wet to the dry seasons increased (Aldrian and Djamil, 2008). This appears to be at least in part consistent with an upward trend of the IOD. While an increasing frequency of extreme events has been reported in the northern parts of South East Asia, decreasing trends in such events are reported in Myanmar (Chang, 2011); see also Figure 14.25.

For a given region, strong seasonality in change is observed. In Peninsular Malaya during the southwest monsoon season, total rainfall and the frequency of wet days decreased, but rainfall intensity increased in much of the region (Deni et al., 2010). During the northeast monsoon, total rainfall, the frequency of extreme rainfall events, and rainfall intensity all increased over the peninsula (Suhaila et al., 2010).

High-resolution model simulations are necessary to resolve complex terrain such as in Southeast Asia (Nguyen et al., 2012; Section 14.2.2.4). In a RCM downscaling simulation using the A1B emission scenario (Chotamonsak et al., 2011), regional average rainfall was projected to increase, consistent with a combination of the 'warmer getting wetter' mechanism (Section 14.3.1), an increase in summer monsoon, though there is a lack of consensus on future ENSO changes. The spatial pattern of change is similar to that projected in the AR4 (Christensen et al., 2007, Section 11.4).

The median increase in temperature over land ranges from $0.8^{\circ} \mathrm{C}$ in RCP2.6 to $3.2^{\circ} \mathrm{C}$ in RCP8.5 by the end of this century (2081-2100). A moderate increase in precipitation is projected for the region: $1 \%$ in RCP2.6 increasing to $8 \%$ in RCP8.5 by 2100 (Table 14.1, Supplementary Material Table 14.SM.1a to 14.SM.1C, Figures 14.27 and Al.64 to Al.65). On islands neighbouring the southeast tropical Indian Ocean, rainfall is projected to decrease during July to November (the IOD prevalent season), consistent with a slower oceanic warming in the east than in the west tropical Indian Ocean, despite little change projected in the IOD (Section 14.3.3).

In summary, warming is very likely to continue with substantial sub-regional variations. There is medium confidence in a moderate increase in rainfall, except on Indonesian islands neighbouring the southeast Indian Ocean. Strong regional variations are expected because of terrain.

\subsubsection{Australia and New Zealand}

The climate of Australia is a mix of tropical and extratropical influences. Northern Australia lies in the tropics and is strongly affected by the Australian monsoon circulation (Section 14.2.2) and ENSO (Section 14.4). Southern Australia extends into the extratropical westerly circulation and is also affected by the middle latitude storm track (Section 14.6.2), the SAM (Section 14.5.2), mid-latitude transient wave propagation, and remotely by the IOD (Section 14.3.3) and ENSO.

Eastern-northeastern Australian rainfall is strongly influenced by the ENSO cycle, with La Niña years typically associated with wet conditions and more frequent and intense tropical cyclones in summer, and $\mathrm{El}$ Niño years with drier than normal conditions, most notably in spring. The SAM plays a significant role in modulating southern Australian rainfall, the positive SAM being associated with generally above-normal rainfall during summer (Hendon et al., 2007; Thompson et al., 2011), but in winter with reduced rainfall, particularly in Southwest 


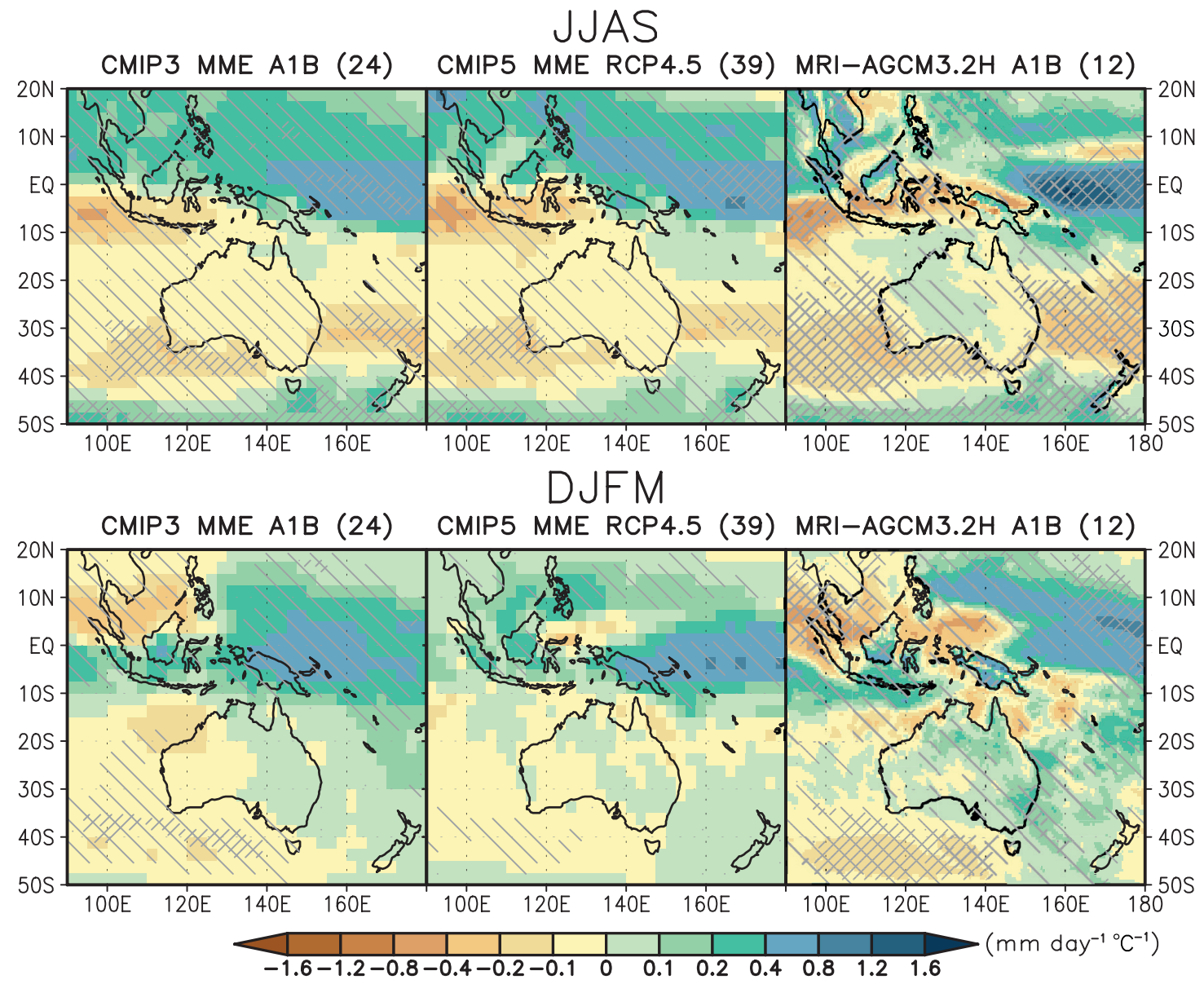

Figure 14.27 | Maps of precipitation changes for Southeast Asia, Australia and New Zealand in 2080-2099 with respect to 1986-2005 in June to September (above) and December to March (below) in the SRES A1B scenario with 24 CMIP3 models (left), and in the RCP4.5 scenario with 39 CMIP5 models (middle). Right figures are the precipitation changes in 2075-2099 with respect to 1979-2003 in the SRES A1B scenario with the 12-member 60- km mesh Meteorological Research Institute (MRI)-Atmospheric General Circulation Model 3.2 (AGCM3.2) multi-physics, multi-sea surface temperature (SST) ensembles (Endo et al., 2012). Precipitation changes are normalized by the global annual mean surface air temperature changes in each scenario. Light hatching denotes where more than $66 \%$ of models (or members) have the same sign with the ensemble mean changes, while dense hatching denotes where more than $90 \%$ of models (or members) have the same sign with the ensemble mean changes.

Western Australia (Hendon et al., 2007; Meneghini et al., 2007; Pezza et al., 2008; Risbey et al., 2009; Cai et al., 2011c). Rossby wavetrains induced by tropical convective anomalies associated with the IOD (Cai et al., 2009), and associated with ENSO through its coherence with the IOD (Cai et al., 2011b) also have a strong impact, leading to lower winter and spring rainfall particularly over Southeastern Australia during positive IOD and El Niño events. Along the eastern seaboard, ETCs (Section 14.6.2) exert a strong influence on the regional climate, while ENSO and other teleconnections play a lesser role (Risbey et al., 2009; Dowdy et al., 2012).

Significant trends have been observed in Australian rainfall over recent decades (Figure 14.25), varying vastly by region and season. Increasing summer rainfall and decreasing temperature trends over northwest Australia have raised the question of whether aerosols originating in the NH play a role (Rotstayn et al., 2007; Shi et al., 2008b; Smith et al., 2008; Rotstayn et al., 2009; Cai et al., 2011d), but there is no consensus at present. By contrast, a prominent rainfall decline has been experienced in austral winter over southwest Western Australia (Cai and Cowan, 2006; Bates et al., 2008) and in mid-to-late autumn over south- eastern Australia (Murphy and Timbal, 2008). Over southwest Western Australia, the decrease in winter rainfall since the late 1960s of about $20 \%$ have led to an even bigger ( $50 \%)$ drop in inflow into dams. The rainfall decline has been linked to changes in large-scale mean sea level pressure (Bates et al., 2008), shifts in synoptic systems (Hope et al., 2006), changes in baroclinicity (Frederiksen and Frederiksen, 2007), the SAM (Cai and Cowan, 2006; Meneghini et al., 2007), land cover changes (Timbal and Arblaster, 2006), anthropogenic forcing (Timbal et al., 2006), Indian Ocean warming (England et al., 2006) and teleconnection to Antarctic precipitation (van Ommen and Morgan, 2010).

Over southeastern Australia, the decreasing rainfall trend is largest in autumn with sustained declines during the drought of 1997-2009, especially in May (Cai and Cowan, 2008; Murphy and Timbal, 2008; Cai et al., 2012a). The exact causes remain contentious, and for the decrease in May, may include ENSO variability and long-term Indian Ocean warming (Cai and Cowan, 2008; Ummenhofer et al., 2009b), a weakening of the subtropical storm track due to decreasing baroclinic instability of the subtropical jet (Frederiksen et al., 2010; Frederiksen et al., 2011a, 2011b) and a poleward shift the ocean-atmosphere circulation 
(Smith and Timbal, 2012; Cai and Cowan, 2013). The well-documented poleward expansion of the subtropical dry zone (Seidel et al., 2008; Johanson and Fu, 2009; Lucas et al., 2012), particularly in April and May, is shown to account for much of the April-May reduction (Cai et al., 2012a). Rainfall trends over southeastern Australia in spring, far weaker but with a signature in the subtropical ridge (Cai et al., 2011a; Timbal and Drosdowsky, 2012), have been shown to be linked with trends and variability in the IOD (Cai et al., 2009; Ummenhofer et al., 2009b). Antarctic proxy data that capture both eastern Australian rainfall and ENSO variability (Vance et al., 2012) show a predominance of El Niño/drier conditions in the 20th century than was the average over the last millennium.

On seasonal to decadal time scales, New Zealand precipitation is modulated by the SAM (Kidston et al., 2009; Thompson et al., 2011), ENSO (Kidson and Renwick, 2002; Ummenhofer and England, 2007) and the IPO (Griffiths, 2007). Increased westerly flow across New Zealand, associated with negative SAM and with El Niño events, leads to increased rainfall and generally lower than normal temperatures in western regions. The positive SAM and La Niña conditions are generally associated with increased rainfall in the north and east of the country, and warmer than normal conditions. On longer time scales, a drying trend since 1979 across much of New Zealand during austral summer is consistent with recent trends in the SAM and to a lesser extent ENSO and the IPO (Griffiths, 2007; Ummenhofer et al., 2009a). In western regions, however, the drying is accompanied by a trend towards increased heavy rainfall (Griffiths, 2007). Temperatures over New Zealand have risen by just under $1^{\circ} \mathrm{C}$ over the past century (Dean and Stott, 2009). The upward trend has been modulated by an increase in the frequency of cool southerly wind flows over the country since the 1950s, without which the observed warming is consistent with largescale anthropogenic forcing (Dean and Stott, 2009).

A recent analysis (Irving et al., 2012; their Figure 9) shows that climate projections over Australia using CMIP5 models, which generally simulate the climate of Australia well (Watterson et al., 2013), are highly consistent with existing CMIP3-derived projections. The projected changes include a further 1.0 to $5.0^{\circ} \mathrm{C}$ temperature rise by the year 2070 (relative to 1990); a long-term drying over southern areas during winter, particularly in the southwest (Figure 14.27), that is consistent with an upward trend of the SAM (Pitman and Perkins, 2008; Shi et al., 2008a; Cai et al., 2011c); a long-term rainfall decline over southern and eastern areas during spring, in part consistent with a upward trend of the IOD index (Smith and Chandler, 2010; Zheng et al., 2010; Weller and Cai, 2013; Zheng et al., 2013). Precipitation change in northeast Australia remains uncertain (Moise et al., 2012), related to the lack of consensus over how ENSO may change (Collins et al., 2010; Section 14.4). In terms of climate extremes, more frequent hot days and nights and less frequent cold days and nights are projected (Alexander and Arblaster, 2009). Changes in the intensity and frequency of extreme rainfall events generally follow the mean rainfall change (Kharin et al., 2007), although there is an increase in most regions in the intensity of short duration extremes (e.g., Alexander and Arblaster, 2009).

For New Zealand, future climate projections suggest further increases in the westerlies in winter and spring, though model biases in jet latitude in the present climate reduce confidence in the detail of future projections (Barnes et al., 2010). The influence of poleward expansion of the subtropical high-pressure belt is projected to lead to drier conditions in parts of the country (Figure 14.27; Table 14.1), and a decrease in westerly wind strength in northern regions. Such projections imply increased seasonality of rainfall in many regions of New Zealand (Reisinger et al., 2010). Both flood and drought occurrence is projected to approximately double over New Zealand during the 21st century, under the SRES A1B scenario. Temperatures are projected to rise at about $70 \%$ of the global rate, because of the buffering effect of the oceans around New Zealand. Temperature rises are projected to be smallest in spring (SON) while the season of greatest warming varies by region around the country. Continued decreases in frost frequency, and increases in the frequency of high-temperature extremes, are expected, but have not been quantified (Reisinger et al., 2010).

In summary, based on understanding of recent trends and on CMIP5 results, it is likely that cool season precipitation will decrease over southern Australia associated in part with trends in the SAM, the IOD and a poleward shift and expansion of the subtropical dry zone. It is very likely that Australia will continue to warm through the 21st century, at a rate similar to the global land surface mean. The frequency of very warm days is very likely to increase through this century, across the whole country.

It is very likely that temperatures will continue to rise over New Zealand. Precipitation is likely to increase in western regions in winter and spring, but the magnitude of change is likely to remain comparable to that of natural climate variability through the rest of the century. In summer and autumn, it is as likely as not that precipitation amounts will change.

\subsubsection{Pacific Islands Region}

The Pacific Islands region includes the northwest tropical Pacific, and the tropical southwest Pacific. North of the Equator, the wet season occurs from May to November. In the south, the wet seasons occurs from November to April.

The phenomena mainly responsible for climate variations in the Pacific Islands are ENSO (Section 14.4), the SPCZ (Section 14.3.1.2), the ITCZ (Section 14.3.1.1) and the WNPSM (Section 14.2.2.5). During El Niño events, the ITCZ and SPCZ move closer to the equator, rainfall decreases in western regions and increases in the central Pacific, and tropical cyclone numbers tend to increase and to occur farther east than normal (Diamond et al., 2012). During La Niña, the western tropical Pacific tends to experience above-average numbers of tropical cyclones (Nicholls et al., 1998; Lavender and Walsh, 2011).

The seasonal evolution of the SPCZ has a strong influence on the seasonality of the climate of the southern tropical Pacific, particularly during the wet season. The SPCZ moves northward during moderate El Niño events and southward during La Niña events (Folland et al., 2002; Vincent et al., 2011). During El Niño events, southwest Pacific Island nations experience an increased occurrence of forest fires and droughts (Salinger et al., 2001; Kumar et al., 2006b), and an increased probability of tropical cyclone damage, as tropical cyclogenesis tends to reside within $6^{\circ}$ to $10^{\circ}$ south of the SPCZ (Vincent et al., 2011). 
Nauru experiences drought during La Niña as the SPCZ and ITCZ move to the west (Brown et al., 2012c). During strong El Niño events (e.g., $1982 / 1983,1997 / 1998)$ the SPCZ undergoes an extreme swing of up to 10 degrees towards the equator and collapses to a more zonally oriented structure (Vincent et al., 2011; Section 14.3.2). The impacts from these zonal SPCZ events are much more severe than those from moderate El Niño events (Vincent et al., 2011; Cai et al., 2012b), and can induce massive droughts and food shortages (Barnett, 2011).

Temperatures have increased at a rate between $0.1^{\circ} \mathrm{C}$ and $0.2^{\circ} \mathrm{C}$ per decade throughout the Pacific Islands during the 20th century (Folland et al., 2003). Changes in temperature extremes have followed those of mean temperatures (Manton et al., 2001; Griffiths et al., 2005). During 1961-2000, locations to the northeast of the SPCZ became wetter, with the largest trends occurring in the eastern Pacific Ocean (east of $160^{\circ} \mathrm{W}$ ), while locations to the southwest of the SPCZ became drier (Griffiths et al., 2003), indicative of a northeastward shift of the SPCZ. Trends in the frequency of rain days were generally similar to those of total annual rainfall (Manton et al., 2001; Griffiths et al., 2003). Since 1980, western Pacific monsoon- and ITCZ-related rain during June to August has decreased (Hennessy et al., 2011).

Future projections for tropical Pacific Island nations are based on direct outputs from a suite of CMIP3 models, updated using CMIP5 wherever available (Brown et al., 2011; Hennessy et al., 2011; Irving et al., 2011; Moise and Delage, 2011; Perkins, 2011; Perkins et al., 2012). These projections carry a large uncertainty, even in the sign of change, as discussed below and as evident in Table 14.1.

Annual average air and sea surface temperature are projected to continue to increase for all tropical Pacific countries. By 2055, under the high $\mathrm{A} 2$ emissions scenario, the increase is projected to be $1^{\circ} \mathrm{C}$ to $2^{\circ} \mathrm{C}$. $A$ rise in the number of hot days and warm nights is also projected, and a decline in cooler weather, as already observed (Manton et al., 2001). For a low-emission scenario, the lower range decreases about $0.5^{\circ} \mathrm{C}$ while the upper range reduces by between $0.2^{\circ} \mathrm{C}$ and $0.5^{\circ} \mathrm{C}$.

To a large extent, the response of the ITCZ, the SPCZ, and the WNPSM to greenhouse warming will determine how rainfall patterns will change in tropical Pacific. In northwestern and near-equatorial regions, rainfall during all seasons is projected to increase in the 21 st century. Wet season increases are consistent with the expected intensification of the WNPSM and the ITCZ (Smith et al., 2012a). For the southwestern tropical Pacific, the CMIP3 and CMIP5 ensemble mean change in summer rainfall is far smaller than the inter-model range (Brown et al., 2012b; Widlansky et al., 2013). There is a projected intensification in the western part of the SPCZ and near the equator with little mean change in SPCZ position (Brown et al., 2012a; Brown et al., 2012b). For the southern group of the Cook Islands, the Solomon Islands, and Tuvalu, average rainfall during the wet season is projected to increase; and for Vanuatu, Tonga, Samoa, Niue, Fiji, a decrease in dry season rainfall is accompanied by an increase in the wet season, indicating an intensified seasonal cycle.

Extreme rainfall days are likely to occur more often in all regions related to an intensification of the ITCZ and the SPCZ (Perkins, 2011). Although the intensification appears to be reproduced in CMIP5 models (Brown et al., 2012a), it has recently been questioned (Widlansky et al., 2013; see Section 14.3.1). There are two competing mechanisms, the 'wet regions getting wetter' and the 'warmest getting wetter, or coldest getting drier' paradigms. These two mechanisms compete within much of the SPCZ region. Based on a multi-model ensemble of 55 greenhouse warming experiments, in which model biases were corrected, tropical SST changes between $2^{\circ} \mathrm{C}$ to $3^{\circ} \mathrm{C}$ resulted in a $5 \%$ decrease of austral summer moisture convergence in the current SPCZ region (Widlansky et al., 2013). This projects a diminished rainy season for most Southwest Pacific island nations. In Samoa and neighbouring islands, summer rainfall may decrease on average by 10 to $20 \%$ during the 21 st century as simulated by the hierarchy of bias-corrected atmospheric model experiments. Less rainfall, combined with increasing surface temperatures and enhanced potential evaporation, could increase the chance for longerterm droughts in the region. Such projections are completely opposite to those based on direct model outputs (Figure 14.27).

Recent downscaling experiments support the above conclusion regarding the impact of biases on the SPCZ change, and suggest that the projected intensification of the ITCZ may have uncertainties of a similar nature (Chapter 7 of Hennessy et al., 2011). In these experiments a bias correction is applied to average sea surface temperatures, and the atmosphere is forced with the 'correct' climatological seasonal cycle together with warming derived from large-scale model outputs. The results show opposite changes in much of the SPCZ and some of the ITCZ regions, resulting in much lower confidence in rainfall projections.

Despite the uncertainty, there is general agreement in model projections regarding an increase in rainfall along the equator (Tables 14.1 and 14.2), and regarding a faster warming rate in the equatorial Pacific than the off-equatorial regions (Xie et al., 2010b). A potential consequence is an increase in the frequency of the zonal SPCZ events (Cai et al., 2012b).

In summary, based on CMIP3 and CMIP5 model projections and recently observed trends, it is very likely that temperatures, including the frequency and magnitude of extreme high temperatures, will continue to increase through the 21 st century. In equatorial regions, the consistency across model projections suggests that rainfall is likely to increase. However, given new model results and physical insights since the $A R 4$, the rainfall outlook is uncertain in regions directly affected by the SPCZ and western portion of the ITCZ.

\subsubsection{Antarctica}

Much of the climate variability of Antarctica is modulated by the Southern Annular Mode (SAM, Section 14.5.2), the high-latitude atmospheric response to ENSO (Section 14.4) and interactions between the two (Stammerjohn et al., 2008; Fogt et al., 2011; see also Sections 2.7 and 10.3.3). Signatures of the SAM and ENSO in Antarctic temperature, snow accumulation and sea ice have been documented by many observational and modelling studies (Bromwich et al., 2004; Guo et al., 2004; Kaspari et al., 2004; van den Broeke and van Lipzig, 2004; Marshall, 2007).

The positive SAM is associated on average with warmer conditions over the Peninsula and colder conditions over East Antarctica, with a mixed 
and generally non-significant impact over West Antarctica (Kwok and Comiso, 2002; Thompson and Solomon, 2002; van den Broeke and van Lipzig, 2004). ENSO is associated with circulation anomalies over the southeast Pacific that primarily affect West Antarctica (Bromwich et al., 2004; Guo et al., 2004; Turner, 2004). ENSO variability tends to produce out-of-phase variations between the western and eastern sectors of West Antarctica (Bromwich et al., 2004; Kaspari et al., 2004), in association with the PSA pattern (Section 14.7.1).

The positive summer/autumn trend in the SAM index in recent decades (Section 14.5.2) has been related to the contrasting temperature trend patterns observed in these two seasons, with warming in the east and north of the Antarctic Peninsula and cooling (or no significant temperature change) over much of East Antarctica (Turner et al., 2005; Thompson et al., 2011). The high polarity of the SAM is also consistent with the significant increase in snow accumulation observed in the southern part of the Peninsula (Thomas et al., 2008).

Unlike the eastern Antarctic Peninsula, its western coast shows maximum warming in austral winter (when the SAM does not exhibit any significant trend), which has been attributed to reduced sea ice concentrations in the Bellingshausen Sea. Recent studies have emphasized the role of tropical SST forcing not directly linked to ENSO to explain the prominent spring- and wintertime atmospheric warming in West Antarctica (Ding et al., 2011; Schneider et al., 2012). There is further evidence of tropical SST influence on Antarctic temperatures and precipitation on decadal to inter-decadal time scales (Monaghan and Bromwich, 2008; Okumura et al., 2012).

Modelling of Antarctic climate remains challenging, in part because of the nature of the high-elevation ice sheet in the east Antarctic and its effects on regional climate (Section 9.4.1.1). Moreover, modelling ice properties themselves, for both land ice and sea ice, is an area that is still developing despite improvements in recent years (Vancoppenolle et al., 2009; Picard et al., 2012; Section 9.4.3). Modelling the role of the stratosphere and of ozone recovery is critical for Antarctic climate, as stratospheric change is intimately linked to trends in the SAM (Section 14.5.2).

The projected easing of the positive SAM trend in austral summer (Section 14.5.2) may act to delay future loss of Antarctic sea ice (Bitz and Polvani, 2012; Smith et al., 2012b). It is unclear what effect ENSO will have on future Antarctic climate change as the ENSO response to climate change remains uncertain (see 12.4.4.1 and 14.5.2 for more information). Seasonally, changes in the strength of the circumpolar westerlies are also expected during the 21st century as a result of changes in the semi-annual oscillation caused by alterations in the mid- to high-latitude temperature gradient in the SH. Bracegirdle et al. (2008) considered modelled circulation changes over the Southern Ocean and found a more pronounced strengthening of the autumn peak of the semi-annual oscillation compared with the spring peak.

Future changes in surface temperature over Antarctica are likely to be smaller than the global mean, and much smaller than those projected for the Arctic, because of the buffering effect of the southern oceans, and the thermal mass of the east Antarctic ice sheet (Section 12.4.6). Warming is likely to bring increased precipitation on average across
Antarctica (Bracegirdle et al., 2008), but the spatial pattern of precipitation change remains uncertain.

In summary, consistency across CMIP5 projections suggests it is very likely that Antarctic temperatures will increase through the rest of the century, but more slowly than the global mean rate of increase (Table 14.1). SSTs of the oceans around Antarctica are likely to rise more slowly than surface air temperature over the Antarctic land mass. As temperatures rise, it is also likely that precipitation will increase (Table 14.1), up to $20 \%$ or more over the East Antarctic. However, given known difficulties associated with correctly modelling Antarctic climate, and uncertainties associated with future SAM and ENSO trends and the extent of Antarctic sea ice, precipitation projections have only medium confidence. 
Table 14.1 | Temperature and precipitation projections by the CMIP5 global models. The figures shown are averages over SREX regions (Seneviratne et al., 2012) of the projections by a set of 42 global models for the RCP4.5 scenario. Added to the SREX regions are a six other regions including the two Polar Regions, the Caribbean, Indian Ocean and Pacific Island States (see Annex I for further details). The 26 SREX regions are: Alaska/NW Canada (ALA), Eastern Canada/Greenland/Iceland (CGI), Western North America (WNA), Central North America (CNA), Eastern North America (ENA), Central America/Mexico (CAM), Amazon (AMZ), NE Brazil (NEB), West Coast South America (WSA), Southeastern South America (SSA), Northern Europe (NEU), Central Europe (CEU), Southern Europe/the Mediterranean (MED), Sahara (SAH), Western Africa (WAF), Eastern Africa (EAF), Southern Africa (SAF), Northern Asia (NAS), Western Asia (WAS), Central Asia (CAS), Tibetan Plateau (TIB), Eastern Asia (EAS), Southern Asia (SAS), Southeastern Asia (SEA), Northern Australia (NAS) and Southern Australia/New Zealand (SAU). The area-mean temperature and precipitation responses are first averaged for each model over the 1986-2005 period from the historical simulations and the 2016-2035, 2046-2065 and 2081-2100 periods of the RCP4.5 experiments. Based on the difference between these two periods, the table shows the 25th, 50th and 75th percentiles, and the lowest and highest response among the 42 models, for temperature in degrees Celsius and precipitation as a percent change. Regions in which the middle half ( 25 to $75 \%$ ) of this distribution is all of the same sign in the precipitation response are coloured light brown for decreasing precipitation and light green for increasing precipitation. Information is provided for land areas contained in the boxes unless otherwise indicated. The temperature responses are averaged over the boreal winter and summer seasons; December, January and February (DJF) and June, July and August (JJA) respectively. The precipitation responses are averaged over half year periods, boreal winter; October, November, December, January, February and March (ONDJFM) and summer; April, May, June, July, August and September (AMJJAS).

\begin{tabular}{|c|c|c|c|c|c|c|c|c|c|c|c|c|}
\hline \multicolumn{3}{|l|}{ RCP4.5 } & \multicolumn{5}{|c|}{ Temperature $\left({ }^{\circ} \mathrm{C}\right)$} & \multicolumn{5}{|c|}{ Precipitation (\%) } \\
\hline REGION & MONTHa & Year & $\min$ & $25 \%$ & $50 \%$ & $75 \%$ & $\max$ & $\min$ & $25 \%$ & $50 \%$ & $75 \%$ & $\max$ \\
\hline \multicolumn{13}{|l|}{ Arctic } \\
\hline \multirow{6}{*}{ (land) } & \multirow{2}{*}{ DJF } & 2065 & 0.4 & 3.0 & 3.4 & 4.5 & 8.0 & 5 & 14 & 17 & 21 & 37 \\
\hline & & 2100 & -0.9 & 3.7 & 5.0 & 6.2 & 10.0 & -2 & 18 & 24 & 30 & 50 \\
\hline & JJA & 2100 & 0.3 & 1.8 & 2.2 & 3.0 & 6.0 & -2 & 10 & 13 & 17 & 39 \\
\hline & \multirow[t]{3}{*}{ Annual } & 2035 & 0.4 & 1.3 & 1.5 & 1.7 & 3.8 & 1 & 5 & 6 & 8 & 20 \\
\hline & & 2065 & 0.3 & 2.4 & 2.8 & 3.5 & 6.4 & 3 & 11 & 13 & 15 & 35 \\
\hline & & 2100 & -0.4 & 3.0 & 3.9 & 4.7 & 7.8 & -2 & 14 & 17 & 21 & 43 \\
\hline \multirow{5}{*}{ (sea) } & \multirow{2}{*}{ JJA } & 2065 & 0.0 & 0.8 & 1.2 & 1.4 & 2.9 & -2 & 9 & 11 & 14 & 23 \\
\hline & & 2100 & -0.3 & 1.2 & 1.5 & 2.1 & 4.0 & -3 & 12 & 16 & 18 & 29 \\
\hline & \multirow[t]{3}{*}{ Annual } & 2035 & 0.2 & 1.5 & 2.0 & 2.3 & 4.7 & 0 & 6 & 8 & 9 & 21 \\
\hline & & 2065 & -0.1 & 2.9 & 3.7 & 4.7 & 7.4 & -1 & 11 & 13 & 20 & 28 \\
\hline & & 2100 & -1.0 & 3.7 & 4.9 & 6.5 & 9.3 & -7 & 16 & 21 & 26 & 37 \\
\hline \multicolumn{13}{|c|}{ High latitudes } \\
\hline \multirow{3}{*}{$\begin{array}{l}\text { Canada/ } \\
\text { Greenland/ } \\
\text { Iceland }\end{array}$} & \multirow[t]{3}{*}{ DJF } & 2035 & -0.2 & 1.2 & 1.7 & 1.9 & 3.1 & 0 & 4 & 5 & 9 & 14 \\
\hline & & 2065 & 0.6 & 2.8 & 3.4 & 3.9 & 6.6 & 3 & 9 & 12 & 15 & 21 \\
\hline & & 2100 & -0.5 & 3.2 & 4.6 & 5.6 & 8.1 & -2 & 11 & 15 & 22 & 32 \\
\hline \multirow[t]{9}{*}{ North Asia } & \multirow[t]{3}{*}{ DJF } & 2035 & 0.5 & 1.1 & 1.5 & 2.2 & 4.0 & 2 & 6 & 8 & 10 & 22 \\
\hline & & 2065 & 1.2 & 2.3 & 3.0 & 3.6 & 6.0 & 5 & 11 & 14 & 18 & 34 \\
\hline & & 2100 & 0.2 & 3.0 & 3.8 & 4.9 & 7.8 & 5 & 13 & 18 & 22 & 44 \\
\hline & \multirow[t]{3}{*}{ JJA } & 2035 & 0.1 & 0.8 & 1.0 & 1.4 & 2.5 & 1 & 2 & 4 & 6 & 16 \\
\hline & & 2065 & 0.8 & 1.5 & 2.0 & 2.7 & 4.4 & -1 & 5 & 8 & 10 & 21 \\
\hline & & 2100 & 0.8 & 1.9 & 2.4 & 3.5 & 5.1 & -3 & 6 & 9 & 12 & 30 \\
\hline & \multirow[t]{3}{*}{ Annual } & 2035 & 0.4 & 1.1 & 1.3 & 1.6 & 3.0 & 1 & 4 & 5 & 7 & 18 \\
\hline & & 2065 & 0.8 & 2.0 & 2.4 & 2.9 & 4.9 & 2 & 8 & 9 & 12 & 25 \\
\hline & & 2100 & 0.2 & 2.5 & 3.2 & 3.8 & 5.8 & 1 & 10 & 12 & 15 & 35 \\
\hline
\end{tabular}


Table 14.1 (continued)

\begin{tabular}{|c|c|c|c|c|c|c|c|c|c|c|c|c|}
\hline \multicolumn{3}{|l|}{ RCP4.5 } & \multicolumn{5}{|c|}{ Temperature $\left({ }^{\circ} \mathrm{C}\right)$} & \multicolumn{5}{|c|}{ Precipitation (\%) } \\
\hline REGION & MONTH ${ }^{\mathrm{a}}$ & Year & $\min$ & $25 \%$ & $50 \%$ & $75 \%$ & $\max$ & $\min$ & $25 \%$ & $50 \%$ & $75 \%$ & $\max$ \\
\hline \multicolumn{13}{|c|}{ North America } \\
\hline \multirow{6}{*}{$\begin{array}{l}\text { Alaska/ } \\
\text { NW Canada }\end{array}$} & \multirow{2}{*}{ DJF } & 2065 & 1.2 & 2.8 & 3.6 & 4.8 & 7.4 & 3 & 9 & 11 & 17 & 29 \\
\hline & & 2100 & 2.3 & 3.5 & 4.8 & 5.9 & 9.7 & 7 & 11 & 17 & 21 & 42 \\
\hline & JJA & 2100 & 0.9 & 1.8 & 2.2 & 3.1 & 5.2 & -2 & 9 & 12 & 16 & 34 \\
\hline & \multirow[t]{3}{*}{ Annual } & 2035 & 0.4 & 1.0 & 1.4 & 1.8 & 2.8 & 0 & 3 & 6 & 7 & 14 \\
\hline & & 2065 & 1.4 & 2.1 & 2.7 & 3.6 & 5.2 & 4 & 8 & 10 & 13 & 28 \\
\hline & & 2100 & 1.7 & 2.5 & 3.5 & 4.3 & 6.7 & 3 & 11 & 14 & 17 & 33 \\
\hline \multirow{6}{*}{$\begin{array}{l}\text { West North } \\
\text { America }\end{array}$} & \multirow[t]{3}{*}{ JJA } & 2035 & 0.3 & 0.9 & 1.1 & 1.3 & 2.1 & -6 & -1 & 1 & 3 & 9 \\
\hline & & 2065 & 0.8 & 1.7 & 2.0 & 2.6 & 3.4 & -7 & -1 & 1 & 4 & 10 \\
\hline & & 2100 & 0.9 & 2.1 & 2.5 & 3.4 & 4.6 & -8 & -1 & 2 & 6 & 10 \\
\hline & \multirow[t]{3}{*}{ Annual } & 2035 & 0.3 & 0.8 & 1.0 & 1.3 & 1.9 & -4 & -1 & 2 & 3 & 6 \\
\hline & & 2065 & 0.9 & 1.7 & 2.0 & 2.5 & 3.4 & -3 & 1 & 3 & 5 & 11 \\
\hline & & 2100 & 1.1 & 2.0 & 2.6 & 3.4 & 4.3 & -4 & 2 & 4 & 6 & 14 \\
\hline \multirow{4}{*}{$\begin{array}{l}\text { Central North } \\
\text { America }\end{array}$} & \multirow[t]{3}{*}{ DJF } & 2035 & -0.1 & 0.7 & 1.1 & 1.6 & 2.9 & -8 & -1 & 1 & 5 & 11 \\
\hline & & 2065 & 0.9 & 1.6 & 2.2 & 2.7 & 4.2 & -7 & 1 & 4 & 7 & 17 \\
\hline & & 2100 & 1.2 & 2.0 & 2.7 & 3.6 & 4.9 & -6 & -1 & 4 & 9 & 18 \\
\hline & JJA & 2035 & 0.3 & 0.8 & 1.1 & 1.4 & 2.3 & -7 & -2 & 0 & 3 & 9 \\
\hline \multirow{8}{*}{$\begin{array}{l}\text { Eastern North } \\
\text { America }\end{array}$} & \multirow{2}{*}{ DJF } & 2065 & 0.9 & 1.7 & 2.4 & 2.8 & 4.1 & -2 & 4 & 7 & 9 & 18 \\
\hline & & 2100 & 0.7 & 2.2 & 2.9 & 3.8 & 4.8 & -4 & 6 & 9 & 12 & 20 \\
\hline & \multirow[t]{3}{*}{ JJA } & 2035 & 0.1 & 0.8 & 1.0 & 1.2 & 1.9 & -4 & 0 & 3 & 5 & 9 \\
\hline & & 2065 & 0.8 & 1.5 & 2.0 & 2.4 & 3.9 & -6 & 2 & 4 & 6 & 14 \\
\hline & & 2100 & 1.0 & 2.0 & 2.5 & 3.1 & 4.8 & -7 & 2 & 5 & 7 & 14 \\
\hline & \multirow[t]{3}{*}{ Annual } & 2035 & 0.4 & 0.8 & 1.1 & 1.3 & 1.9 & -4 & 1 & 3 & 5 & 9 \\
\hline & & 2065 & 1.0 & 1.7 & 2.1 & 2.4 & 3.5 & -1 & 3 & 5 & 7 & 14 \\
\hline & & 2100 & 1.0 & 2.1 & 2.7 & 3.1 & 4.2 & -2 & 4 & 7 & 9 & 14 \\
\hline
\end{tabular}


Table 14.1 (continued)

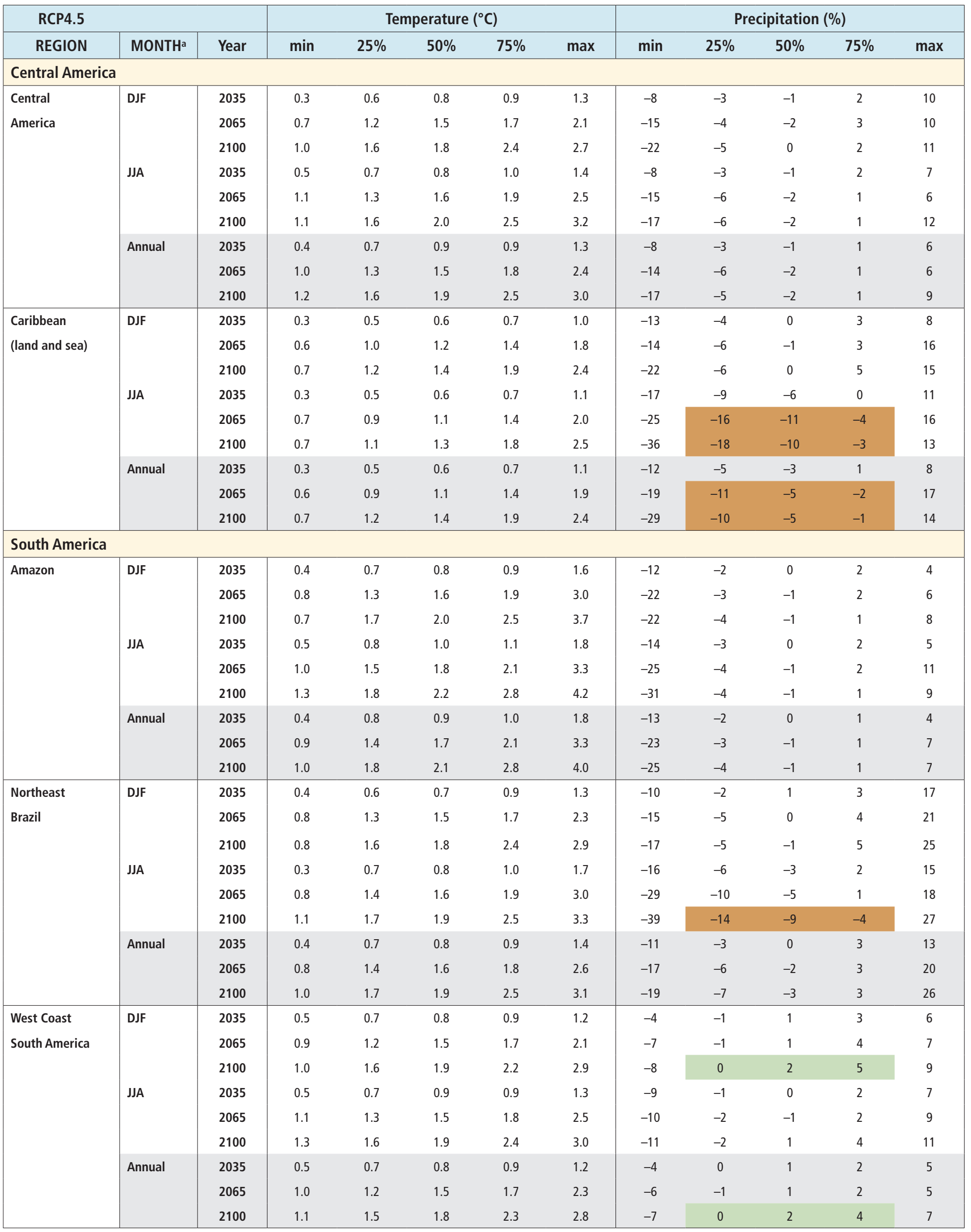


Table 14.1 (continued)

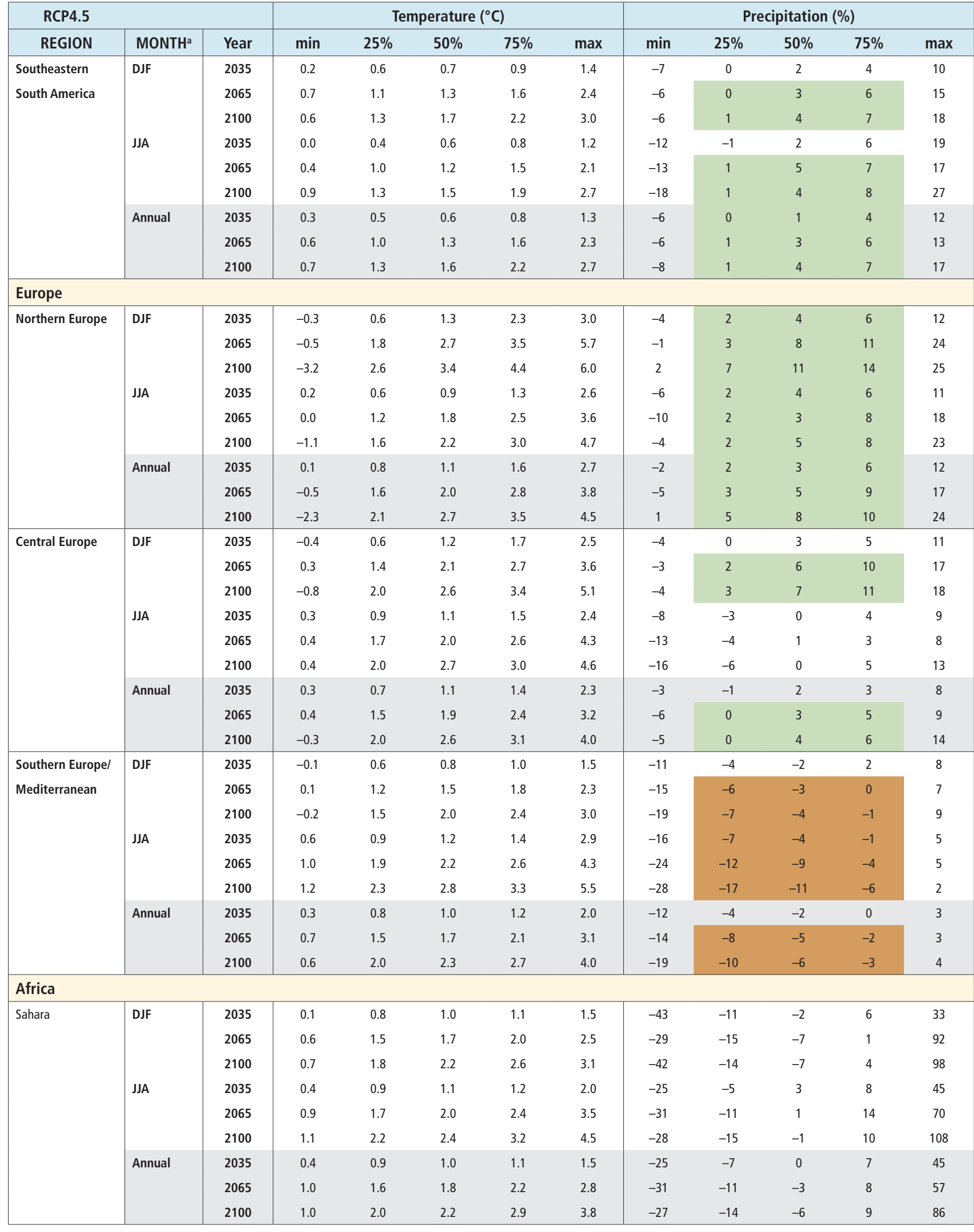


Table 14.1 (continued)

\begin{tabular}{|c|c|c|c|c|c|c|c|c|c|c|c|c|}
\hline \multicolumn{3}{|l|}{ RCP4.5 } & \multicolumn{5}{|c|}{ Temperature $\left({ }^{\circ} \mathrm{C}\right)$} & \multicolumn{5}{|c|}{ Precipitation (\%) } \\
\hline REGION & MONTHa & Year & $\min$ & $25 \%$ & $50 \%$ & $75 \%$ & $\max$ & $\min$ & $25 \%$ & $50 \%$ & $75 \%$ & $\max$ \\
\hline \multirow[t]{9}{*}{ West Africa } & \multirow[t]{3}{*}{ DJF } & 2035 & 0.4 & 0.8 & 0.9 & 1.0 & 1.3 & -5 & -1 & 2 & 3 & 9 \\
\hline & & 2065 & 0.9 & 1.4 & 1.6 & 1.9 & 2.7 & -10 & 1 & 4 & 5 & 7 \\
\hline & & 2100 & 1.3 & 1.7 & 2.0 & 2.5 & 3.6 & -5 & 1 & 4 & 6 & 11 \\
\hline & \multirow[t]{3}{*}{ JJA } & 2035 & 0.6 & 0.7 & 0.8 & 0.9 & 1.2 & -4 & 0 & 1 & 2 & 6 \\
\hline & & 2065 & 1.0 & 1.3 & 1.5 & 1.9 & 2.6 & -9 & -1 & 2 & 3 & 6 \\
\hline & & 2100 & 0.9 & 1.6 & 1.8 & 2.6 & 3.3 & -12 & 0 & 2 & 4 & 9 \\
\hline & \multirow[t]{3}{*}{ Annual } & 2035 & 0.6 & 0.7 & 0.8 & 0.9 & 1.2 & -4 & -1 & 1 & 3 & 8 \\
\hline & & 2065 & 1.1 & 1.3 & 1.5 & 1.9 & 2.5 & -10 & 0 & 2 & 4 & 6 \\
\hline & & 2100 & 1.0 & 1.6 & 1.9 & 2.6 & 3.2 & -8 & 1 & 3 & 4 & 8 \\
\hline \multirow[t]{9}{*}{ East Africa } & \multirow[t]{3}{*}{ DJF } & 2035 & 0.4 & 0.7 & 0.8 & 1.0 & 1.2 & -4 & -1 & 1 & 5 & 10 \\
\hline & & 2065 & 0.8 & 1.3 & 1.5 & 1.8 & 2.5 & -3 & -1 & 3 & 7 & 19 \\
\hline & & 2100 & 1.0 & 1.6 & 1.9 & 2.4 & 3.2 & -6 & -1 & 5 & 10 & 25 \\
\hline & \multirow[t]{3}{*}{ JJA } & 2035 & 0.5 & 0.7 & 0.9 & 1.0 & 1.2 & -8 & -3 & 0 & 2 & 12 \\
\hline & & 2065 & 0.8 & 1.4 & 1.6 & 1.9 & 2.4 & -10 & -4 & 1 & 3 & 18 \\
\hline & & 2100 & 0.7 & 1.7 & 2.0 & 2.5 & 3.1 & -12 & -4 & 0 & 5 & 19 \\
\hline & \multirow[t]{3}{*}{ Annual } & 2035 & 0.5 & 0.7 & 0.8 & 0.9 & 1.2 & -5 & -2 & 1 & 3 & 10 \\
\hline & & 2065 & 1.0 & 1.3 & 1.6 & 1.9 & 2.4 & -6 & -2 & 1 & 6 & 17 \\
\hline & & 2100 & 1.0 & 1.6 & 2.0 & 2.5 & 3.1 & -7 & -2 & 2 & 8 & 21 \\
\hline \multirow{9}{*}{$\begin{array}{l}\text { Southern } \\
\text { Africa }\end{array}$} & \multirow[t]{3}{*}{ DJF } & 2035 & 0.6 & 0.7 & 0.9 & 1.1 & 1.3 & -11 & -4 & -2 & 0 & 3 \\
\hline & & 2065 & 1.0 & 1.4 & 1.7 & 2.0 & 2.6 & -19 & -5 & -3 & -1 & 4 \\
\hline & & 2100 & 1.1 & 1.8 & 2.1 & 2.7 & 3.3 & -19 & -7 & -3 & 1 & 5 \\
\hline & \multirow[t]{3}{*}{ JJA } & 2035 & 0.5 & 0.8 & 0.9 & 1.0 & 1.5 & -18 & -9 & -4 & -1 & 9 \\
\hline & & 2065 & 1.1 & 1.5 & 1.7 & 2.0 & 2.5 & -29 & -13 & -8 & -3 & 4 \\
\hline & & 2100 & 1.4 & 1.8 & 2.1 & 2.6 & 3.3 & -29 & -18 & -9 & -3 & 12 \\
\hline & \multirow[t]{3}{*}{ Annual } & 2035 & 0.6 & 0.8 & 0.9 & 1.0 & 1.4 & -13 & -5 & -2 & 0 & 4 \\
\hline & & 2065 & 1.1 & 1.5 & 1.7 & 2.1 & 2.6 & -15 & -7 & -4 & -1 & 4 \\
\hline & & 2100 & 1.4 & 1.8 & 2.1 & 2.7 & 3.3 & -20 & -7 & -5 & -1 & 5 \\
\hline \multirow{9}{*}{$\begin{array}{l}\text { West Indian } \\
\text { Ocean }\end{array}$} & \multirow[t]{3}{*}{ DJF } & 2035 & 0.3 & 0.5 & 0.6 & 0.7 & 1.0 & -10 & 0 & 2 & 3 & 10 \\
\hline & & 2065 & 0.6 & 1.0 & 1.1 & 1.3 & 1.8 & -10 & -1 & 2 & 5 & 13 \\
\hline & & 2100 & 0.8 & 1.2 & 1.4 & 1.8 & 2.3 & -9 & -1 & 2 & 6 & 22 \\
\hline & \multirow[t]{3}{*}{ JJA } & 2035 & 0.4 & 0.5 & 0.6 & 0.7 & 1.0 & -5 & -1 & 2 & 5 & 12 \\
\hline & & 2065 & 0.6 & 0.9 & 1.1 & 1.3 & 1.8 & -7 & -1 & 1 & 5 & 12 \\
\hline & & 2100 & 0.7 & 1.2 & 1.4 & 1.8 & 2.3 & -7 & 0 & 2 & 5 & 19 \\
\hline & \multirow[t]{3}{*}{ Annual } & 2035 & 0.3 & 0.5 & 0.6 & 0.7 & 1.0 & -5 & 1 & 2 & 3 & 7 \\
\hline & & 2065 & 0.6 & 1.0 & 1.1 & 1.3 & 1.8 & -4 & -1 & 2 & 4 & 11 \\
\hline & & 2100 & 0.8 & 1.2 & 1.4 & 1.8 & 2.2 & -5 & 0 & 2 & 5 & 19 \\
\hline Asia & & & & & & & & & & & & \\
\hline West Asia & DJF & 2035 & 0.0 & 0.8 & 1.1 & 1.4 & 1.8 & -12 & 0 & 3 & 6 & 14 \\
\hline & & 2065 & 0.5 & 1.5 & 1.9 & 2.3 & 3.2 & -10 & -1 & 2 & 7 & 21 \\
\hline & & 2100 & 0.6 & 1.9 & 2.4 & 2.9 & 3.8 & -11 & -3 & 4 & 9 & 20 \\
\hline & JJA & 2035 & 0.2 & 0.9 & 1.1 & 1.3 & 2.1 & -10 & -2 & 1 & 5 & 55 \\
\hline & & 2065 & 1.1 & 1.7 & 2.1 & 2.6 & 4.0 & -20 & -6 & -3 & 2 & 51 \\
\hline & & 2100 & 1.2 & 2.0 & 2.7 & 3.4 & 4.7 & -29 & -6 & -1 & 4 & 60 \\
\hline & Annual & 2035 & 0.1 & 0.9 & 1.0 & 1.2 & 1.8 & -9 & -2 & 3 & 4 & 27 \\
\hline & & 2065 & 0.7 & 1.7 & 1.9 & 2.3 & 3.2 & -12 & -2 & 0 & 4 & 27 \\
\hline & & 2100 & 0.9 & 2.1 & 2.5 & 3.1 & 4.1 & -19 & -2 & 1 & 6 & 28 \\
\hline
\end{tabular}


Table 14.1 (continued)

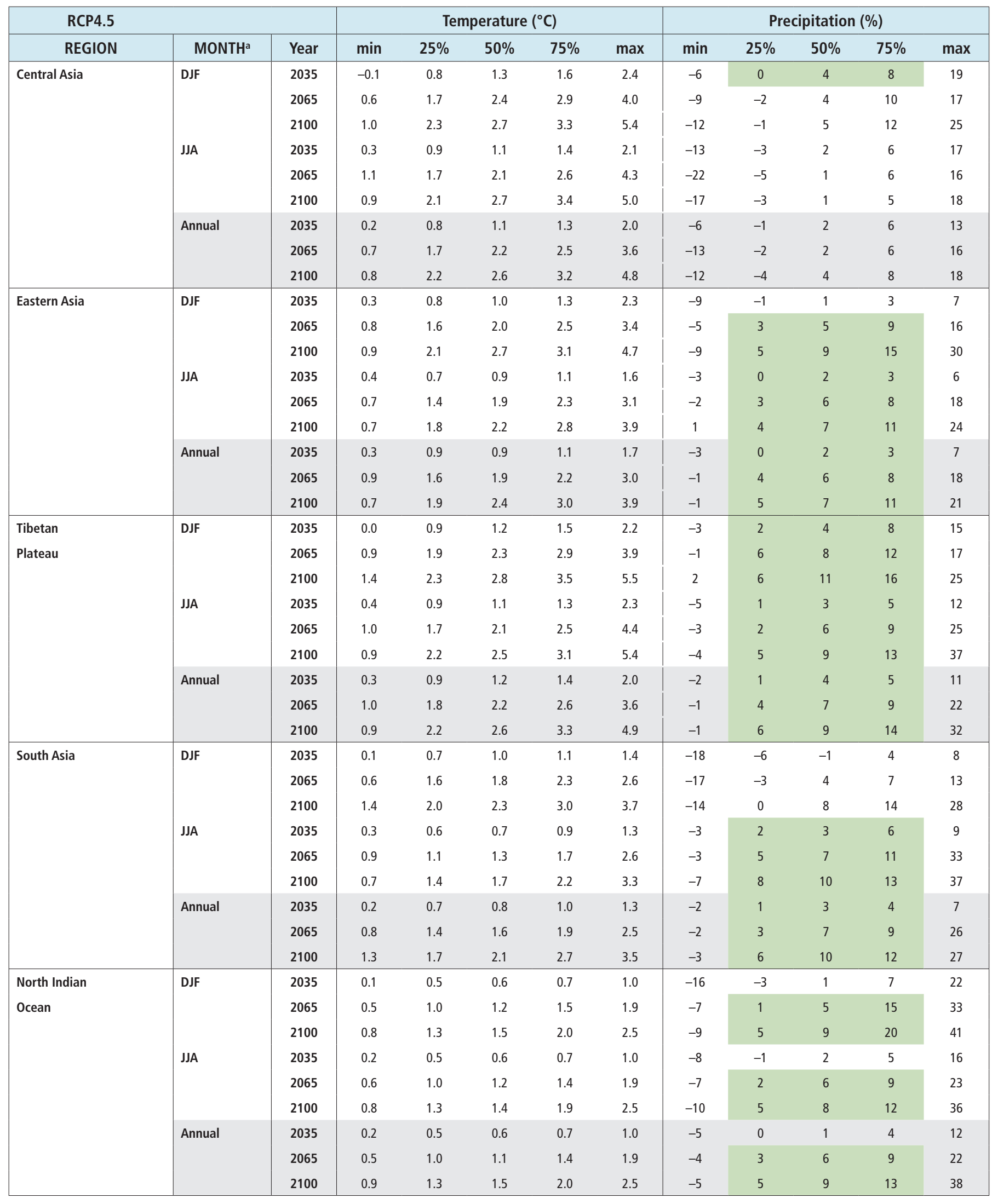


Table 14.1 (continued)

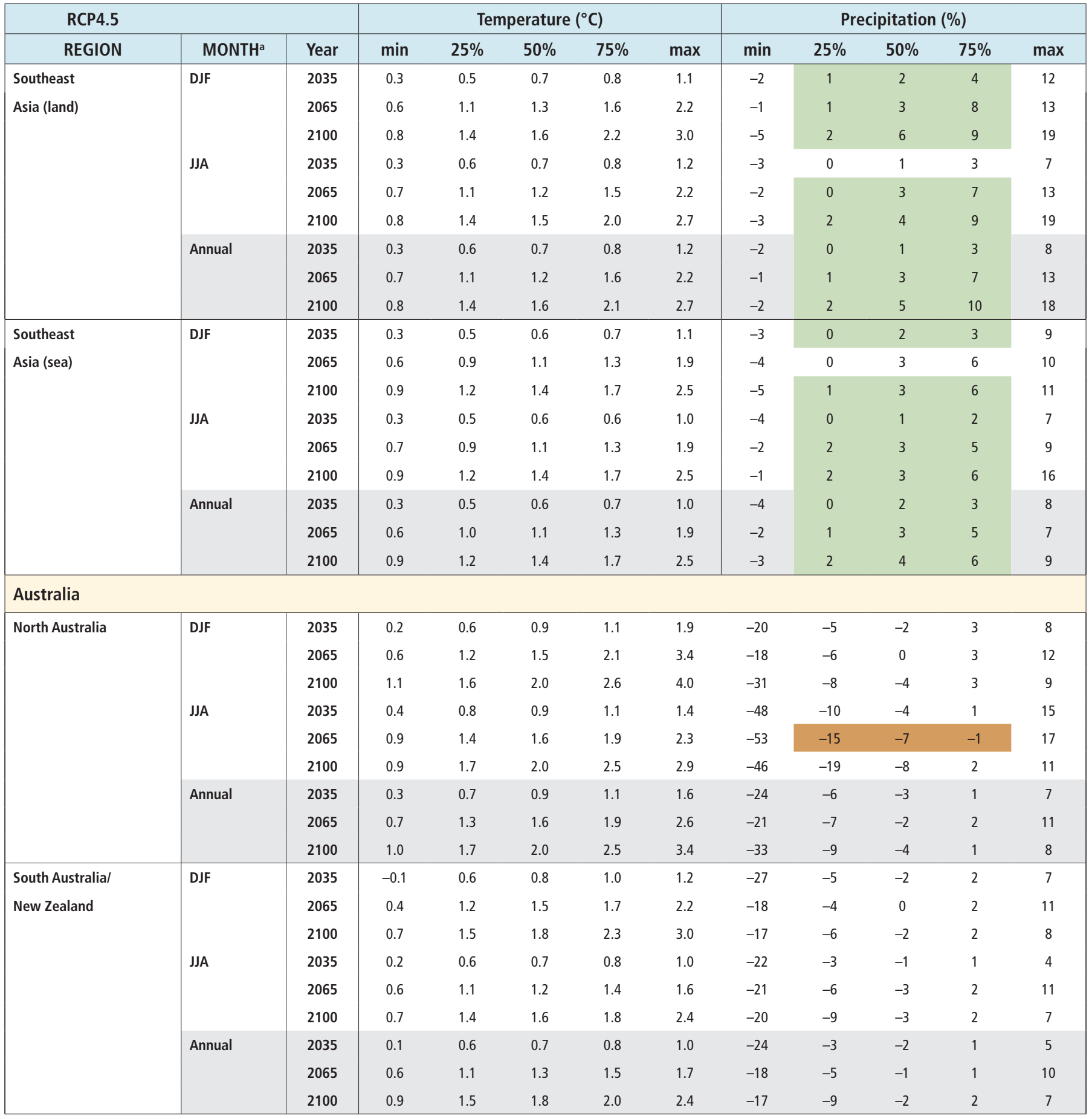


Table 14.1 (continued)

\begin{tabular}{|c|c|c|c|c|c|c|c|c|c|c|c|c|}
\hline \multicolumn{3}{|l|}{ RCP4.5 } & \multicolumn{5}{|c|}{ Temperature $\left({ }^{\circ} \mathrm{C}\right)$} & \multicolumn{5}{|c|}{ Precipitation (\%) } \\
\hline REGION & MONTHa & Year & $\min$ & $25 \%$ & $50 \%$ & $75 \%$ & $\max$ & $\min$ & $25 \%$ & $50 \%$ & $75 \%$ & $\max$ \\
\hline \multicolumn{13}{|l|}{ The Pacific } \\
\hline \multirow{6}{*}{$\begin{array}{l}\text { Northern } \\
\text { Tropical Pacific }\end{array}$} & \multirow{2}{*}{ DJF } & 2065 & 0.7 & 1.0 & 1.1 & 1.4 & 1.9 & -4 & -2 & 1 & 6 & 12 \\
\hline & & 2100 & 0.9 & 1.2 & 1.4 & 1.7 & 2.4 & -6 & -1 & 1 & 5 & 20 \\
\hline & JJA & 2100 & 0.8 & 1.1 & 1.4 & 1.8 & 2.6 & -11 & -1 & 2 & 4 & 16 \\
\hline & \multirow[t]{3}{*}{ Annual } & 2035 & 0.3 & 0.5 & 0.6 & 0.7 & 1.0 & -8 & -2 & 1 & 3 & 7 \\
\hline & & 2065 & 0.6 & 1.0 & 1.1 & 1.3 & 1.9 & -7 & -1 & 1 & 4 & 9 \\
\hline & & 2100 & 0.9 & 1.2 & 1.4 & 1.7 & 2.4 & -8 & 0 & 1 & 4 & 18 \\
\hline \multirow{5}{*}{ Equatorial Pacific } & \multirow{2}{*}{ JJA } & 2065 & 0.7 & 1.0 & 1.1 & 1.4 & 2.3 & 0 & 11 & 15 & 25 & 143 \\
\hline & & 2100 & 0.5 & 1.2 & 1.5 & 1.8 & 2.9 & -19 & 13 & 23 & 33 & 125 \\
\hline & \multirow[t]{3}{*}{ Annual } & 2035 & 0.1 & 0.5 & 0.7 & 0.7 & 1.1 & -11 & 3 & 7 & 12 & 40 \\
\hline & & 2065 & 0.7 & 1.0 & 1.2 & 1.4 & 2.3 & -1 & 7 & 12 & 24 & 194 \\
\hline & & 2100 & 0.5 & 1.2 & 1.4 & 1.8 & 2.9 & -23 & 13 & 19 & 29 & 225 \\
\hline \multirow[t]{4}{*}{ Southern Pacific } & \multirow[t]{3}{*}{ DJF } & 2035 & 0.3 & 0.4 & 0.5 & 0.6 & 0.9 & -7 & -1 & 1 & 2 & 6 \\
\hline & & 2065 & 0.6 & 0.8 & 1.0 & 1.2 & 1.5 & -22 & 0 & 2 & 4 & 6 \\
\hline & & 2100 & 0.8 & 1.0 & 1.3 & 1.5 & 2.0 & -24 & -1 & 3 & 5 & 8 \\
\hline & JJA & 2035 & 0.3 & 0.4 & 0.5 & 0.6 & 0.9 & -10 & 0 & 1 & 3 & 8 \\
\hline
\end{tabular}

\section{Antarctica}

\begin{tabular}{|c|c|c|c|c|c|c|c|c|c|c|c|c|}
\hline \multirow[t]{9}{*}{ (land) } & \multirow[t]{3}{*}{ DJF } & 2035 & 0.1 & 0.5 & 0.6 & 0.8 & 1.3 & -3 & 1 & 3 & 4 & 8 \\
\hline & & 2065 & 0.1 & 1.0 & 1.3 & 1.6 & 2.3 & -7 & 3 & 5 & 8 & 14 \\
\hline & & 2100 & 0.5 & 1.5 & 1.7 & 2.1 & 3.1 & -5 & 4 & 8 & 10 & 17 \\
\hline & \multirow[t]{3}{*}{ JJA } & 2035 & -0.5 & 0.6 & 0.8 & 0.9 & 1.8 & -3 & 2 & 5 & 6 & 13 \\
\hline & & 2065 & -0.1 & 1.2 & 1.4 & 1.8 & 2.5 & 1 & 6 & 8 & 13 & 16 \\
\hline & & 2100 & -0.3 & 1.5 & 1.9 & 2.4 & 3.8 & -1 & 9 & 12 & 15 & 23 \\
\hline & \multirow[t]{3}{*}{ Annual } & 2035 & -0.1 & 0.5 & 0.7 & 0.9 & 1.3 & -3 & 2 & 4 & 5 & 9 \\
\hline & & 2065 & 0.0 & 1.1 & 1.3 & 1.7 & 2.3 & -3 & 4 & 7 & 10 & 14 \\
\hline & & 2100 & 0.1 & 1.5 & 1.8 & 2.3 & 3.2 & -3 & 7 & 9 & 13 & 21 \\
\hline \multirow[t]{9}{*}{ (sea) } & \multirow[t]{3}{*}{ DJF } & 2035 & -0.3 & 0.2 & 0.4 & 0.5 & 0.7 & -1 & 1 & 3 & 3 & 5 \\
\hline & & 2065 & -0.4 & 0.5 & 0.6 & 0.9 & 1.3 & 0 & 3 & 4 & 5 & 8 \\
\hline & & 2100 & -0.3 & 0.6 & 0.9 & 1.2 & 1.8 & 0 & 4 & 5 & 7 & 11 \\
\hline & \multirow[t]{3}{*}{ JJA } & 2035 & -0.7 & 0.4 & 0.6 & 1.0 & 1.9 & 0 & 2 & 2 & 4 & 5 \\
\hline & & 2065 & -0.6 & 0.7 & 1.1 & 1.6 & 3.3 & 2 & 4 & 5 & 7 & 10 \\
\hline & & 2100 & -0.8 & 1.1 & 1.4 & 2.2 & 3.8 & 3 & 5 & 7 & 10 & 13 \\
\hline & \multirow[t]{3}{*}{ Annual } & 2035 & -0.4 & 0.3 & 0.5 & 0.7 & 1.3 & 0 & 2 & 2 & 4 & 5 \\
\hline & & 2065 & -0.5 & 0.5 & 0.8 & 1.2 & 2.3 & 2 & 3 & 4 & 6 & 9 \\
\hline & & 2100 & -0.5 & 0.8 & 1.2 & 1.7 & 2.6 & 1 & 4 & 6 & 9 & 12 \\
\hline
\end{tabular}

Notes:

a Precipitation changes cover 6 months; ONDJFM and AMJJAS for winter and summer (Northern Hemisphere). 
Table 14.2 Assessed confidence (high, medium, low) in climate projections of regional temperature and precipitation change from the multi-model ensemble of CMIP5 models for the RCP4.5 scenario. Column 1 refers to the SREX regions (cf. Seneviratne et al., 2012, page 12. The region's coordinates can be found from their online Appendix 3.A) and six additional regions including the two polar regions, the Caribbean, Indian Ocean and Pacific Island States (see Annex I for further details). Columns 2 to 4 show confidence in models' ability to simulate present-day mean temperature and precipitation as well as the most important phenomena for that region based on Figures 9.39, 9.40, and 9.45. In column 4, the individual phenomena are listed, with associated confidence levels shown below, in the same order as the phenomena. Note that only phenomena assessed in Figure 9.45 are listed. Column 5 is an interpretation of the relevance of the main climate phenomena for future regional climate change, based on Table 14.3. Note that the SREX regions are smaller than the regions listed in Table 14.3. Columns 6 and 7 express confidence in projected temperature and precipitation changes, based solely on model agreement for 2080-2099 vs. 1985-2005, as listed in Table 14.1 and in the maps shown in Annex I. The confidence is assessed for two periods for temperature (DJF and JJA) and two-half year periods for precipitation (October to March and April to September). When the projections are consistent with no significant change, it is marked by an asterisk (*) and the assigned confidence is medium. Further details on how confidence levels have been assigned are provided in the Supplementary Material (Section 14.SM.6.1).

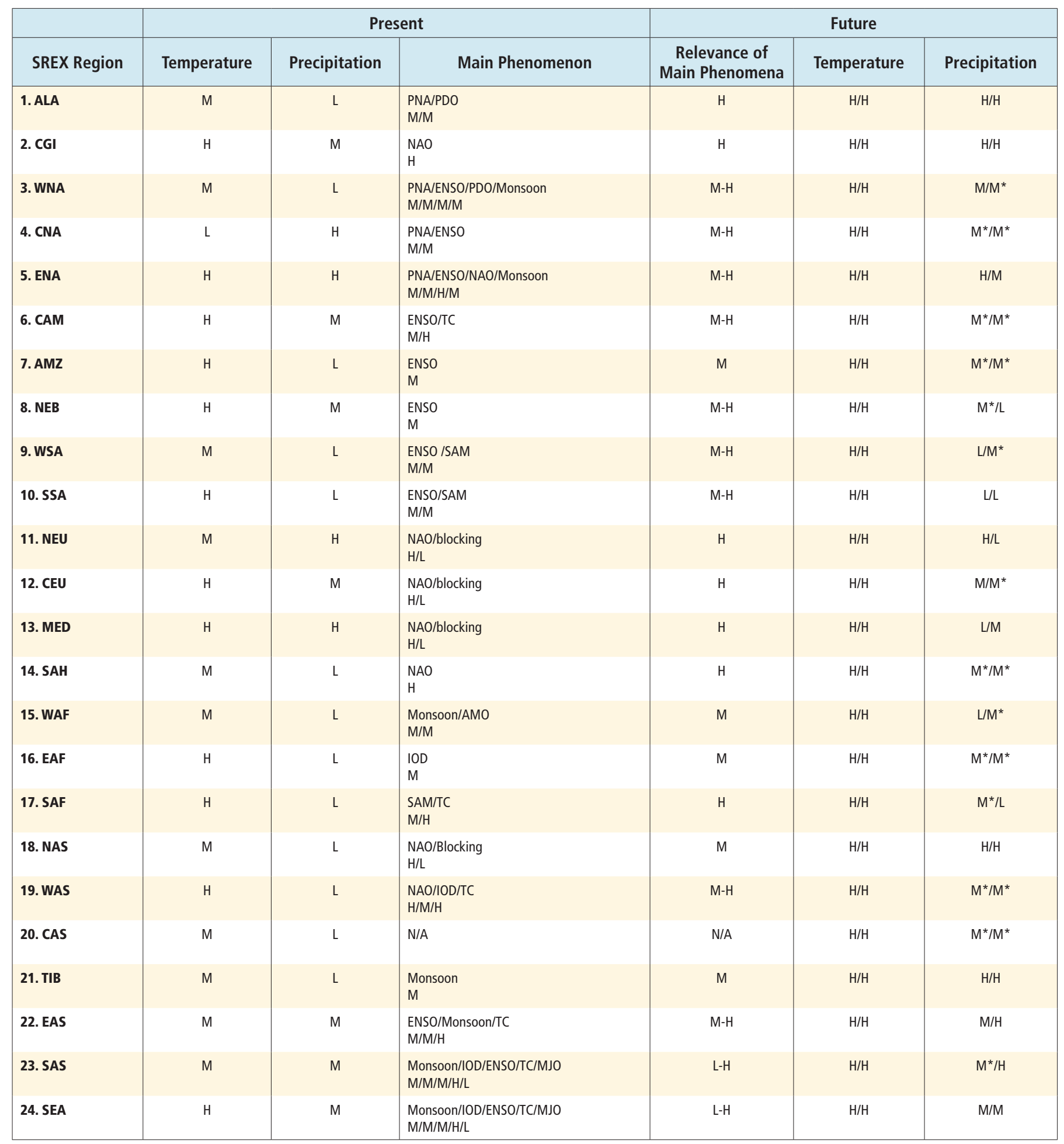


Table 14.2 (continued)

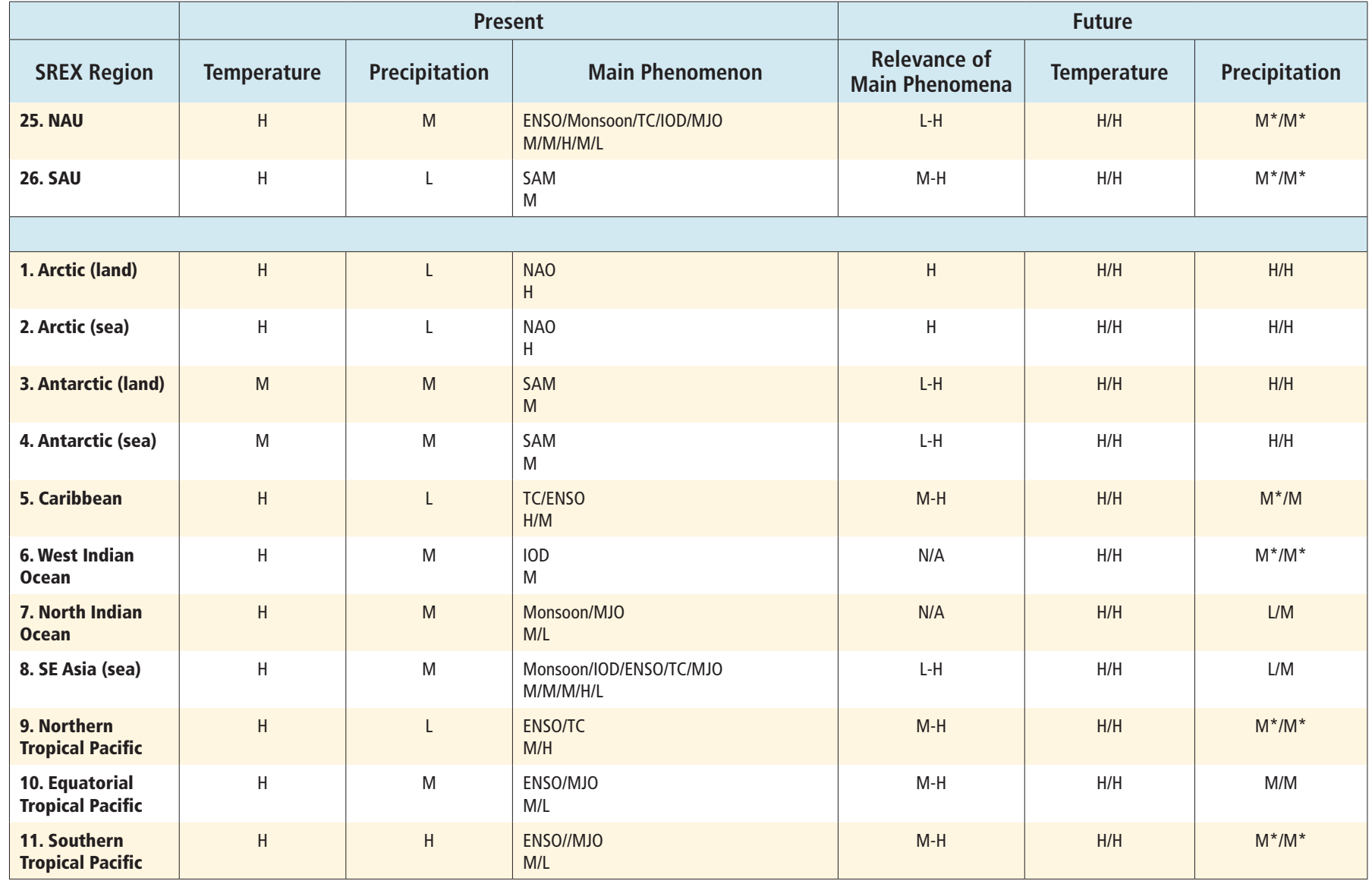




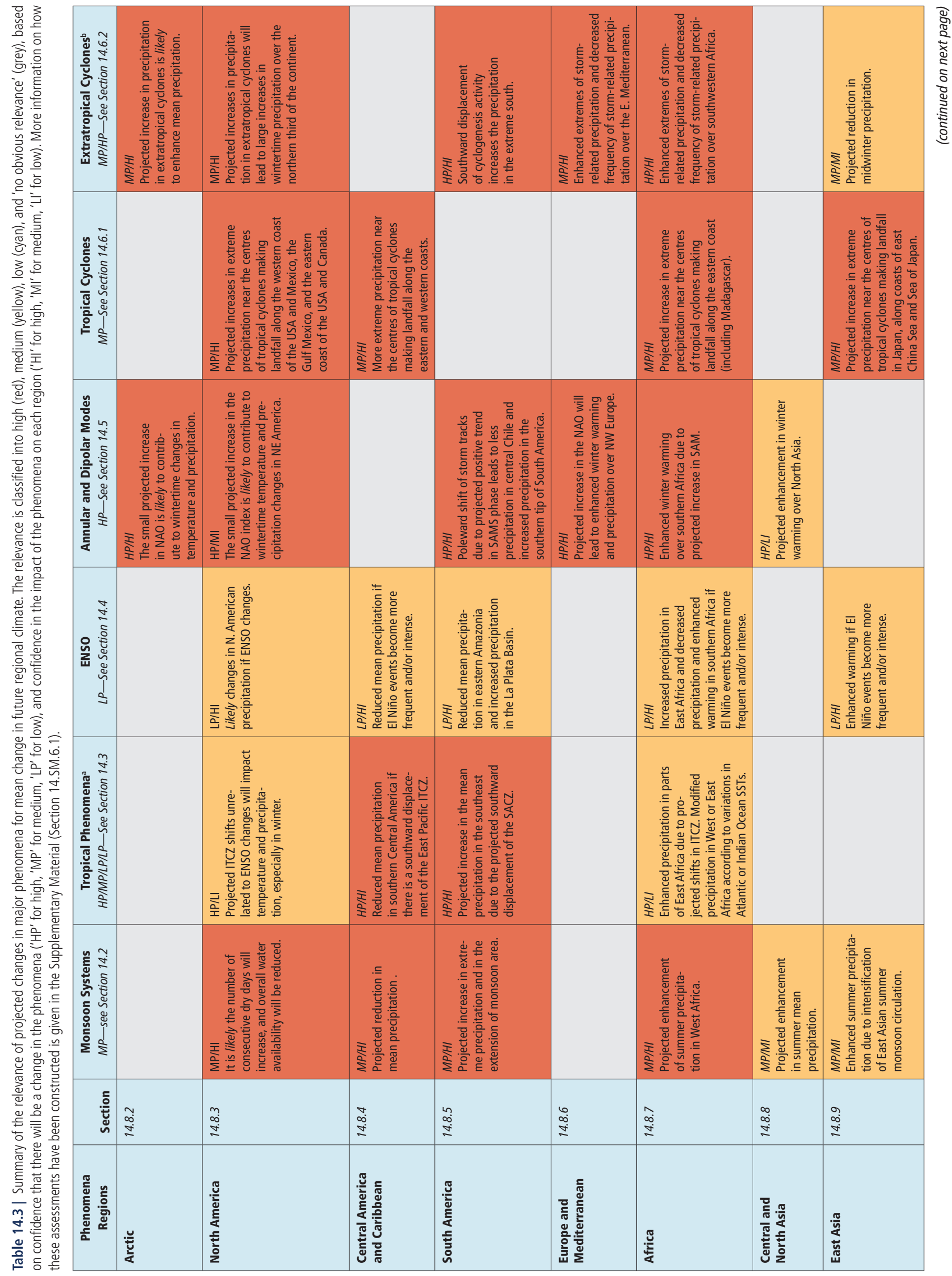




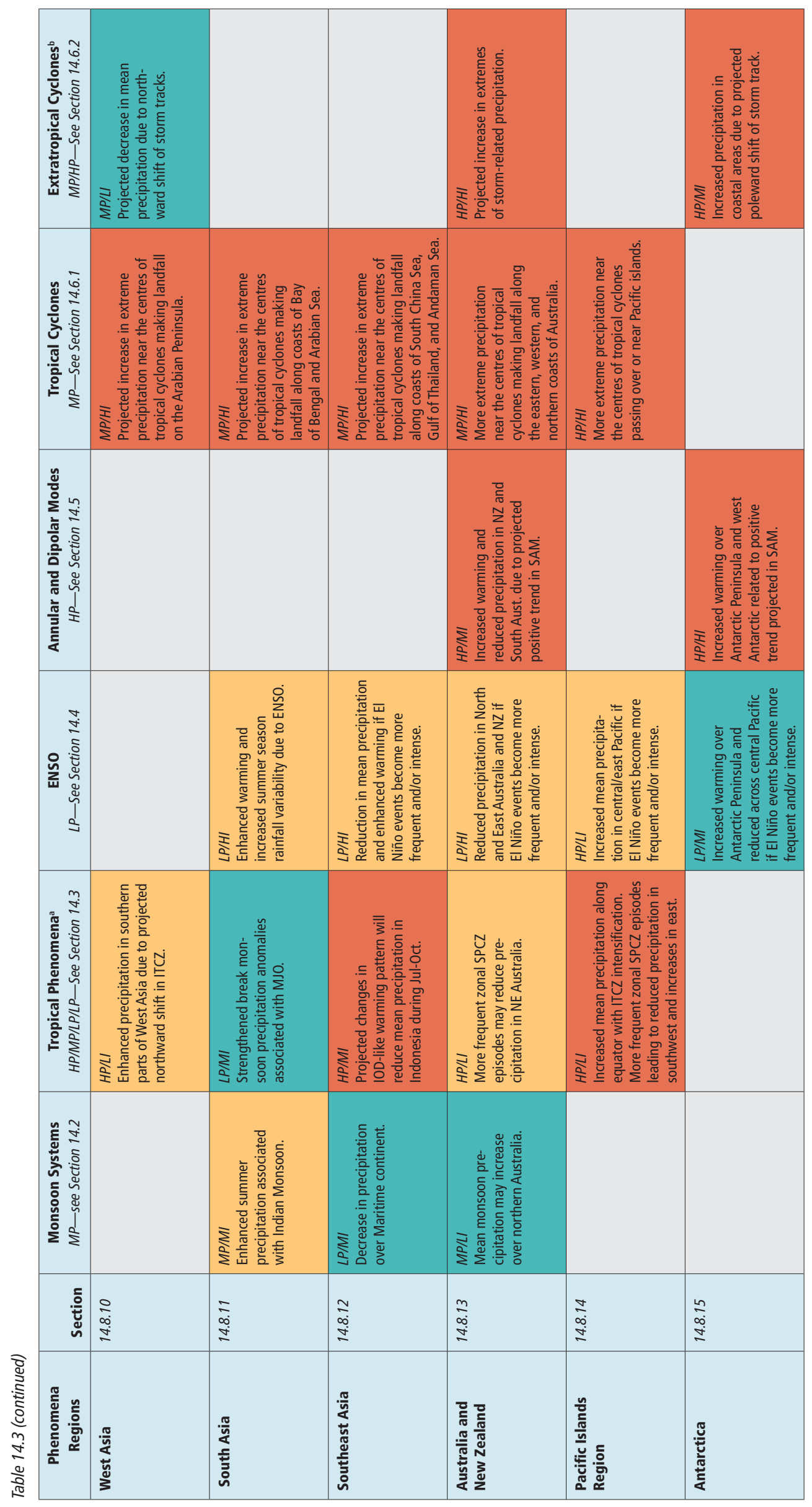




\section{References}

Abram, N. J., M. K. Gagan, J. E. Cole, W. S. Hantoro, and M. Mudelsee, 2008: Recent intensification of tropical climate variability in the Indian Ocean. Nature Geosci., 1, 849-853.

Ackerley, D., B. B. B. Booth, S. H. E. Knight, E. J. Highwood, D. J. Frame, M. R. Allen, and D. P. Rowell, 2011: Sensitivity of twentieth-century Sahel rainfall to sulfate aerosol and $\mathrm{CO}_{2}$ forcing. J. Clim., 24, 4999-5014.

Aldrian, E., and Y. S. Djamil, 2008: Spatio-temporal climatic change of rainfall in east Java Indonesia. Int. J. Climatol., 28, 435-448.

Alexander, L. V., and J. M. Arblaster, 2009: Assessing trends in observed and modelled climate extremes over Australia in relation to future projections. Int. J. Climatol., 29, 417-435.

Alexander, L. V., et al., 2006: Global observed changes in daily climate extremes of temperature and precipitation. J. Geophys. Res. Atmos., 111, D05109.

Alexander, M., D. Vimont, P. Chang, and J. Scott, 2010: The impact of extratropical atmospheric variability on ENSO: Testing the seasonal footprinting mechanism using coupled model experiments. J. Clim., 23, 2885-2901.

Alexander, M., I. Blade, M. Newman, J. Lanzante, N. Lau, and J. Scott, 2002: The atmospheric bridge: The influence of ENSO teleconnections on air-sea interaction over the global oceans. J. Clim., 15, 2205-2231.

Alexander, M. A., 2010: Extratropical air-sea interaction, SST variability and the Pacific Decadal Oscillation (PDO). In: Climate Dynamics: Why Does Climate Vary? [D. S. a. F. Bryan (ed.)]. American Geophysical Union, Washingon, DC, pp. 123-148.

Allan, R., and B. Soden, 2008: Atmospheric warming and the amplification of precipitation extremes. Science, 321, 1481-1484.

Alory, G., S. Wijfels, and G. Meyers, 2007: Observed temperature trends in the Indian Ocean over 1960-1999 and associated mechanisms. Geophys. Res. Lett., 34, L02606.

Alpert, P., S. Krichak, H. Shafir, D. Haim, and I. Osetinsky, 2008: Climatic trends to extremes employing regional modeling and statistical interpretation over the $\mathrm{E}$. Mediterranean. Global Planet. Change, 63, 163-170.

Alpert, P., et al., 2002: The paradoxical increase of Mediterranean extreme daily rainfall in spite of decrease in total values. Geophys. Res. Lett., 29, 31-34.

AlSarmi, S., and R. Washington, 2011: Recent observed climate change over the Arabian Peninsula. J. Geophys. Res. Atmos., 116, D11109.

Alves, L. M., and J. A. Marengo, 2010: Assessment of regional seasonal predictability using the PRECIS regional climate modeling system over South America. Theor. Appl. Climatol., 100, 337-350.

Amador, J. A., E. J. Alfaro, O. G. Lizano, and V. O. Magana, 2006: Atmospheric forcing of the eastern tropical Pacific: A review. Prog. Oceanogr., 69, 101-142.

AMAP, 2011: Snow, Water, Ice and Permafrost in the Arctic (SWIPA): Climate Change and the Cryosphere, Arctic Monitoring and Assessment Programme, Oslo, Norway, $538 \mathrm{pp}$.

Ambaum, M., B. Hoskins, and D. Stephenson, 2001: Arctic oscillation or North Atlantic oscillation? J. Clim., 14, 3495-3507.

Ambaum, M. H. P., 2008: Unimodality of wave amplitude in the Northern Hemisphere. J. Atmos. Sci., 65, 1077-1086.

An, S.-I., J.-W. Kim, S.-H. Im, B.-M. Kim, and J.-H. Park, 2011: Recent and future sea surface temperature trends in the tropical Pacific warm pool and cold tongue regions. Clim. Dyn., doi:10.1007/s00382-011-1129-7.

An, S. I., and B. Wang, 2000: Interdecadal change of the structure of the ENSO mode and its impact on the ENSO frequency. J. Clim., 13, 2044-2055.

An, S. I., and F. F. Jin, 2000: An Eigen analysis of the interdecadal changes in the structure and frequency of ENSO mode. Geophys. Res. Lett., 27, 2573-2576.

Anderson, B., J. Wang, G. Salvucci, S. Gopal, and S. Islam, 2010: Observed trends in summertime precipitation over the southwestern United States. J. Clim., 23, 1937-1944.

Anderson, B. T., 2003: Tropical Pacific sea surface temperatures and preceding sea level pressure anomalies in the subtropical North Pacific. J. Geophys. Res. Atmos., 108, 4732.

Anderson, B. T., 2011: Near-term increase in frequency of seasonal temperature extremes prior to the 2 degree C global warming target. Clim. Change, 108, 581-589.

Annamalai, H., K. Hamilton, and K. R. Sperber, 2007: The South Asian summer monsoon and its relationship with ENSO in the IPCC AR4 simulations. J. Clim., 20, 1071-1092.
Annamalai, H., J. Hafner, K. P. Sooraj, and P. Pillai, 2013: Global warming shifts monsoon circulation, drying South Asia. J. Clim., 26, 2701-2718.

Anstey, J. A., and T. G. Shepherd, 2008: Response of the northern stratospheric polar vortex to the seasonal alignment of QBO phase transitions. Geophys. Res. Lett., 35, L22810.

Anstey, J. A., et al., 2013: Multi-model analysis of Northern Hemisphere winter blocking, Part I: Model biases and the role of resolution. J. Geophys. Res. Atmos., 118, doi: 10.1002/jgrd.50231.

Arblaster, J. M., G. A. Meehl, and D. J. Karoly, 2011: Future climate change in the Southern Hemisphere: Competing effects of ozone and greenhouse gases. Geophys. Res. Lett., 38, L02701.

Arriaga-Ramirez, S., and T. Cavazos, 2010: Regional trends of daily precipitation indices in northwest Mexico and southwest United States. J. Geophys. Res., 115, D144111.

Ashfaq, M., S. Ying, T. Wen-wen, R. J. Trapp, G. Xueijie, J. S. Pal, and N. S. Diffenbaugh, 2009: Suppression of South Asian summer monsoon precipitation in the 21st century. Geophys. Res. Lett., doi:10.1029/2008gl036500.

Ashok, K., S. K. Behera, S. A. Rao, H. Y. Weng, and T. Yamagata, 2007: El Nino Modoki and its possible teleconnection. J. Geophys. Res. Oceans, 112, C11007.

Athanasiadis, P. J., J. M. Wallace, and J. J. Wettstein, 2010: Patterns of wintertime jet stream variability and their relation to the storm tracks. J. Atmos. Sci, 67, 1361-138.

Bader, J., M. D. S. Mesquita, K. I. Hodges, N. Keenlyside, S. Osterhus, and M. Miles, 2011: A review on Northern Hemisphere sea-ice, storminess and the North Atlantic Oscillation: Observations and projected changes. Atmos. Res., 101, 809-834.

Baines, P. G., and C. K. Folland, 2007: Evidence for a rapid global climate shift across the late 1960s. J. Clim., 20, 2721-2744.

Baldwin, M., D. Stephenson, and I. Jolliffe, 2009: Spatial weighting and iterative projection methods for EOFs. J. Clim., 22, 234-243.

Baldwin, M. P., and D. W. J. Thompson, 2009: A critical comparison of stratospheretroposphere coupling indices. Q. J. R. Meteorol. Soc., 135, 1661-1672.

Baldwin, M. P., et al., 2001: The quasi-biennial oscillation. Rev. Geophys., 39, 179229.

Barnes, E., J. Slingo, and T. Woollings, 2012: A methodology for the comparison of blocking climatologies across indices, models and climate scenarios. Clim. Dyn., 38, 2467-2481.

Barnes, E. A., and D. L. Hartmann, 2012: Detection of Rossby wave breaking and its response to shifts of the midlatitude jet with climate change. J. Geophys. Res. Atmos., 117, D09117.

Barnes, E. A., and L. Polvani, 2013: Response of the midlatitude jets and of their variability to increased greenhouse gases in the CMIP5 models. J. Clim., 26, 7117-7135.

Barnes, E. A., D. L. Hartmann, D. M. W. Frierson, and J. Kidston, 2010: Effect of latitude on the persistence of eddy-driven jets. Geophys. Res. Lett., 37, L11804.

Barnett, J., 2011: Dangerous climate change in the Pacific Islands: Food production and food security. Region. Environ. Change, 11, S229-S237.

Barriopedro, D., R. Garcia-Herrera, A. R. Lupo, and E. Hernandez, 2006: A climatology of Northern Hemisphere blocking. J. Clim., 19, 1042-1063.

Barriopedro, D., R. García-Herrera, J. F. González-Rouco, and R. M. Trigo, 2010: Application of blocking diagnosis methods to General Circulation Models. Part II: Model simulations. Clim. Dyn., 35, 1393-1409.

Barros, V. R., M. Doyle, and I. Camilloni, 2008: Precipitation trends in southeastern South America: Relationship with ENSO phases and the low-level circulation. Theor. Appl. Climatol., 93, 19-33.

Bates, B., P. Hope, B. Ryan, I. Smith, and S. Charles, 2008: Key findings from the Indian Ocean Climate Initiative and their impact on policy development in Australia. Clim. Change, 89, 339-354.

Bates, S. C., 2010: Seasonal influences on coupled ocean-atmosphere variability in the tropical Atlantic ocean. J. Clim., 23, 582-604.

Beck, C., J. Grieser, and B. Rudolf, 2005: A new monthly precipitation climatology for the global land areas for the period 1951 to 2000. In: Climate Status Report 2004. German Weather Service, Offenbach, Germany, pp. 181-190. 
Becker, A., P. Finger, A. Meyer-Christoffer, B. Rudolf, K. Schamm, U. Schneider, and M. Ziese, 2013: A description of the global land-surface precipitation data products of the Global Precipitation Climatology Centre with sample applications including centennial (trend) analysis from 1901-present. Earth Syst. Sci. Data, 5, 71-99.

Bell, C. J., L. J. Gray, A. J. Charlton-Perez, M. M. Joshi, and A. A. Scaife, 2009: Stratospheric communication of El Niño teleconnections to European winter. J. Clim., 22, 4083-4096.

Bender, M. A., T. R. Knutson, R. E. Tuleya, J. J. Sirutis, G. A. Vecchi, S. T. Garner, and I. M. Held, 2010: Modeled impact of anthropogenic warming on the frequency of intense Atlantic hurricanes. Science, 327, 454-458.

Bengtsson, L., K. I. Hodges, and E. Roeckner, 2006: Storm tracks and climate change. J. Clim., 19, 3518-3543.

Bengtsson, L., K. I. Hodges, and N. Keenlyside, 2009: Will extratropical storms intensify in a warmer climate? J. Clim., 22, 2276-2301.

Bengtsson, L., K. I. Hodges, M. Esch, N. Keenlyside, L. Kornblueh, J.-J. Luo, and T. Yamagata, 2007: How may tropical cyclones change in a warmer climate? Tellus A, 59, 539-561.

Berckmans, J., T. Woollings, M.-E. Demory, P.-L. Vidale, and M. Roberts, 2013: Atmospheric blocking in a high resolution climate model: Influences of mean state, orography and eddy forcing. Atmos. Sci. Lett., 14, 34-40.

Berrisford, P., B. J. Hoskins, and E. Tyrlis, 2007: Blocking and Rossby wave-breaking on the dynamical tropopause in the Southern Hemisphere. J. Atmos. Sci., 64, 2881-2898.

Bhend, J., and H. von Storch, 2008: Consistency of observed winter precipitation trends in northern Europe with regional climate change projections. Clim. Dyn., $31,17-28$.

Biasutti, M., and A. Giannini, 2006: Robust Sahel drying in response to late 20th century forcings. Geophys. Res. Lett., 33, L11706.

Biasutti, M., and A. H. Sobel, 2009: Delayed seasonal cycle and African monsoon in a warmer climate. Geophys. Res. Lett., 36, L23707.

Biasutti, M., A. H. Sobel, and S. J. Camargo, 2009: The role of the Sahara Low in summertime Sahel rainfall variability and change in the CMIP3 models. J. Clim., 22, 5755-5771.

Biasutti, M., I. Held, A. Sobel, and A. Giannini, 2008: SST forcings and Sahel rainfall variability in simulations of the twentieth and twenty-first centuries. J. Clim., 21, 3471-3486.

Bitz, C. M., and L. M. Polvani, 2012: Antarctic climate response to stratospheric ozone depletion in a fine resolution ocean climate model. Geophys. Res. Lett., 39, L20705.

Bjerknes, J., 1969: Atmospheric teleconnections from the Equatorial Pacific. Mon. Weather Rev., 97, 163-172.

Black, E., 2009: The impact of climate change on daily precipitation statistics in Jordan and Israel. Atmos. Sci. Lett., 10, 192-200.

Black, E., J. Slingo, and K. Sperber, 2003: An observational study of the relationship between excessively strong short rains in coastal East Africa and Indian Ocean SST. Mon. Weather Rev., 131, 74-94.

Blázquez, J., and M. Nuñez, 2012: Analysis of uncertainties in future climate projections for South America: Comparison of WCRP-CMIP3 and WCRP-CMIP5 models. Clim. Dyn., doi:10.1007/s00382-012-1489-7, 1-18.

Blázquez, J., M. N. Nuñez, and S. Kusunoki, 2012: Climate projections and uncertainties over South America from MRI/JMA global model experiments. Atmos. Clim. Sci, 2, 381-400.

Bluthgen, J., R. Gerdes, and M. Werner, 2012: Atmospheric response to the extreme Arctic sea ice conditions in 2007. Geophys. Res. Lett., 39, L02707.

Boer, G., 2009: Changes in interannual variability and decadal potential predictability under global warming. J. Clim., 22, 3098-3109.

Boer, G. J., and K. Hamilton, 2008: QBO influence on extratropical predictive skill. Clim. Dyn., 31, 987-1000.

Bollasina, M., and Y. Ming, 2013: The general circulation model precipitation bias over the southwestern equatorial Indian Ocean and its implications for simulating the South Asian monsoon. Clim. Dyn., 40, 823-838.

Bollasina, M. A., Y. Ming, and V. Ramaswamy, 2011: Anthropogenic aerosols and the weakening of the south Asian summer monsoon. Science, 334, 502-505.

Bombardi, R. J., and L. M. V. Carvalho, 2009: IPCC global coupled model simulations of the South America monsoon system. Clim. Dyn., 33, 893-916.

Boo, K. O., G. Martin, A. Sellar, C. Senior, and Y. H. Byun, 2011: Evaluating the East Asian monsoon simulation in climate models. J. Geophys. Res., 116, D01109.
Booth, B. B. B., N. J. Dunstone, P. R. Halloran, T. Andrews, and N. Bellouin, 2012: Aerosols implicated as a prime driver of twentieth-century North Atlantic climate variability. Nature, 484, 228-232.

Bracegirdle, T. J., and D. B. Stephenson, 2012: Higher precision estimates of regional polar warming by ensemble regression of climate model projections. Clim. Dyn., 39, 2805-2821.

Bracegirdle, T. J., W. M. Connolley, and J. Turner, 2008: Antarctic climate change over the twenty first century. J. Geophys. Res., 113, D03103.

Bracegirdle, T. J., et al., 2013: Assessment of surface winds over the Atlantic, Indian, and Pacific Ocean sectors of the Southern Ocean in CMIP5 models: Historical bias, forcing response, and state dependence. J. Geophys. Res. Atmos., 118, 547-562.

Braganza, K., J. Gergis, S. Power, J. Risbey, and A. Fowler, 2009: A multiproxy index of the El Niño-Southern Oscillation, AD 1525-1982. J. Geophys. Res. Atmos., 114, D05106.

Brandefelt, J., 2006: Atmospheric modes of variability in a changing climate. J. Clim., 19, 5934-5943.

Branstator, G., and F. Selten, 2009: "Modes of Variability" and Climate Change. J. Clim., 22, 2639-2658.

Breugem, W., W. Hazeleger, and R. Haarsma, 2006: Multimodel study of tropical Atlantic variability and change. Geophys. Res. Lett., doi:10.1029/2006GL027831.

Breugem, W., W. Hazeleger, and R. Haarsma, 2007: Mechanisms of northern tropical Atlantic variability and response to CO2 doubling. J. Clim., doi:DOI 10.1175/ JCLI4137.1, 2691-2705.

Bromwich, D. H., A. J. Monaghan, and Z. C. Guo, 2004: Modeling the ENSO modulation of Antarctic climate in the late 1990s with the polar MM5. J. Clim., 17, 109-132.

Brown, J., A. Moise, and R. Colman, 2012a: The South Pacific Convergence Zone in CMIP5 simulations of historical and future climate. Clim. Dyn., doi:10.1007/ s00382-012-1591-x, 1-19.

Brown, J., A. Moise, and F. Delage, 2012b: Changes in the South Pacific Convergence Zone in IPCC AR4 future climate projections. Clim. Dyn., 39, 1-19.

Brown, J., S. Power, F. Delage, R. Colman, A. Moise, and B. Murphy, 2011: Evaluation of the South Pacific Convergence Zone in IPCC AR4 climate model simulations of the twentieth century. J. Clim., 24, 1565-1582.

Brown, J., et al., 2012c: Implications of CMIP3 model biases and uncertainties for climate projections in the western tropical Pacific. Clim. Change, doi:10.1007/ s10584-012-0603-5, 1-15.

Brown, R., and P. Mote, 2009: The response of Northern Hemisphere snow cover to a changing climate. J. Clim., doi:10.1175/2008JCLI2665.1, 2124-2145.

Budikova, D., 2009: Role of Arctic sea ice in global atmospheric circulation: A review. Global Planet. Change, 68, 149-163.

Buehler, T., C. C. Raible, and T. F. Stocker, 2011: The relationship of winter season North Atlantic blocking frequencies to extreme cold and dry spells in the ERA40. Tellus $A, 63,212-222$.

Bulic, I., and F. Kucharski, 2012: Delayed ENSO impact on spring precipitation over the North/Atlantic European region. Clim. Dyn., 38, 2593-2612.

Bulic, I., C. Brankovic, and F. Kucharski, 2012: Winter ENSO teleconnections in a warmer climate. Clim. Dyn., 38, 1593-1613.

Bunge, L., and A. J. Clarke, 2009: A verified estimation of the El Nino index Nino-3.4 since 1877. J. Clim., 22, 3979-3992.

Butchart, N., et al., 2006: Simulations of anthropogenic change in the strength of the Brewer-Dobson circulation. Clim. Dyn., 27, 727-741.

Butler, A. H., D. W. J. Thompson, and R. Heikes, 2010: The steady-state atmospheric circulation response to climate change-like thermal forcings in a simple General Circulation Model. J. Clim., 23, 3474-3496.

Caesar, J., et al., 2011: Changes in temperature and precipitation extremes over the Indo-Pacific region from 1971 to 2005. Int. J. Climatol., 31, 791-801.

Cai, W., and T. Cowan, 2008: Dynamics of late autumn rainfall reduction over southeastern Australia. Geophys. Res. Lett., 35, L09708.

Cai, W., and T. Cowan, 2013: Southeast Australia autumn rainfall reduction: A climate-change induced poleward shift of ocean-atmosphere circulation. J. Clim., 26, 189-205.

Cai, W., T. Cowan, and A. Sullivan, 2009: Recent unprecedented skewness towards positive Indian Ocean Dipole occurrences and its impact on Australian rainfall. Geophys. Res. Lett., 36, L11705.

Cai, W., P. van Rensch, and T. Cowan, 2011a: Influence of global-scale variability on the subtropical ridge over southeast Australia. J. Clim., 24, 6035-6053. 
Cai, W., T. Cowan, and M. Thatcher, 2012a: Rainfall reductions over Southern Hemisphere semi-arid regions: The role of subtropical dry zone expansion. Sci. Rep., 2, doi: 10.1038/srep00702.

Cai, W., P. van Rensch, T. Cowan, and H. H. Hendon, 2011 b: Teleconnection pathways of ENSO and the IOD and the mechanisms for impacts on Australian rainfall. $J$. Clim., 24, 3910-3923.

Cai, W., P. van Rensch, S. Borlace, and T. Cowan, 2011c: Does the Southern Annular Mode contribute to the persistence of the multidecade-long drought over southwest Western Australia? Geophys. Res. Lett., 38, L14712.

Cai, W., T. Cowan, A. Sullivan, J. Ribbe, and G. Shi, 2011d: Are anthropogenic aerosols responsible for the northwest Australia summer rainfall increase? A CMIP3 perspective and implications. J. Clim., 24, 2556-2564.

Cai, W., et al., 2012b: More extreme swings of the South Pacific convergence zone due to greenhouse warming. Nature, 488, 365-369.

Cai, W. J., and T. Cowan, 2006: SAM and regional rainfall in IPCC AR4 models: Can anthropogenic forcing account for southwest Western Australian winter rainfal reduction? Geophys. Res. Lett., 33, doi: 10.1029/2006gl028037.

Cai, W. J., A. Sullivan, and T. Cowan, 2011e: Interactions of ENSO, the IOD, and the SAM in CMIP3 Models. J. Clim., 24, 1688-1704.

Camargo, S., M. Ting, and Y. Kushnir, 2012: Influence of local and remote SST on North Atlantic tropical cyclone potential intensity Clim. Dyn., 40, 1515-1529.

Campbell, J. D., M. A. Taylor, T. S. Stephenson, R. A. Watson, and F. S. Whyte, 2010: Future climate of the Caribbean from a regional climate model. Int. J. Climatol. 31, 1866-1878.

Cane, M. A., et al., 1997: Twentieth-century sea surface temperature trends. Science, 275, 957-960.

Carrera, M. L., R. W. Higgins, and V. E. Kousky, 2004: Downstream weather impacts associated with atmospheric blocking over the northeast Pacific. J. Clim., 17, 4823-4839.

Carril, A. F., et al., 2012: Performance of a multi-RCM ensemble for South Eastern South America. Clim. Dyn., 39, 2747-2768.

Carton, J., and B. Huang, 1994: Warm events in the tropical Atlantic. J. Phys. Oceanogr., 24, 888-903.

Carvalho, L. M. V., C. Jones, and T. Ambrizzi, 2005: Opposite phases of the antarctic oscillation and relationships with intraseasonal to interannual activity in the tropics during the austral summer. J. Clim., 18, 702-718.

Carvalho, L. M. V., A. E. Silva, C. Jones, B. Liebmann, P. L. Silva Dias, and H. R. Rocha, 2011: Moisture transport and intraseasonal variability in the South America Monsoon System. Clim. Dyn., 36, 1865-1880.

Cassou, C., and L. Terray, 2001: Dual influence of Atlantic and Pacific SST anomalies on the North Atlantic/Europe winter climate. Geophys. Res. Lett., 28, 3195-3198.

Cassou, C., C. Deser, and M. A. Alexander, 2007: Investigating the impact of reemerging sea surface temperature anomalies on the winter atmospheric circulation over the North Atlantic. J. Clim., 20, 3510-3526.

Castro, C. L., R. A. Pielke Sr., and J. O. Adegoke, 2007: Investigation of the summer climate of the contiguous United States and Mexico using the Regional Atmospheric Modeling System (RAMS). Part I: Model climatology (1950-2002). J. Clim., 20, 3844-3865

Casty, C., C. C. Raible, T. F. Stocker, H. Wanner, and J. Luterbacher, 2007: A European pattern climatology 1766-2000. Clim. Dyn., 29, 791-805.

Cattiaux, J., H. Douville, and Y. Peings, 2013: European temperatures in CMIP5: Origins of present-day biases and future uncertainties. Clim. Dyn., doi:10.1007/ s00382-013-1731-y, 1-19.

Catto, J. L., L. C. Shaffrey, and K. I. Hodges, 2011: Northern Hemisphere extratropical cyclones in a warming climate in the HiGEM High-Resolution Climate Model. J. Clim., 24, 5336-5352.

Cavalcanti, I. F. A., and M. H. Shimizu, 2012: Climate fields over South America and variability of SACZ and PSA in HadGEM-ES. Am. J. Clim. Change, 1, 132-144.

Cavazos, T., C. Turrent, and D. P. Lettenmaier, 2008: Extreme precipitation trends associated with tropical cyclones in the core of the North American monsoon. Geophys. Res. Lett., 35, doi: 10.1029/2008GL035832.

Cerezo-Mota, R., M. Allen, and R. Jones, 2011: Mechanisms controlling precipitation in the northern portion of the North American monsoon. J. Clim., 24, 2771-2783.

Chadwick, R., I. Boutle, and G. Martin, 2013: Spatial patterns of precipitation change in CMIP5: Why the rich don't get richer in the tropics. J. Clim., 26, 3803-3822.

Chang, C.-H., 2011: Preparedness and storm hazards in a global warming world: Lessons from Southeast Asia. Nat. Hazards, 56, 667-679.
Chang, C., J. Chiang, M. Wehner, A. Friedman, and R. Ruedy, 2011: Sulfate aerosol control of tropical Atlantic climate over the twentieth century. J. Clim., 24, 2540-2555.

Chang, E. K. M., Y. Guo, and X. Xia, 2012: CMIP5 multimodel ensemble projection of storm track change under global warming. J. Geophys. Res. Atmos., 117, doi: 10.1029/2012jd018578.

Chang, P., et al., 2006: Climate fluctuations of tropical coupled systems - The role of ocean dynamics. J. Clim., 19, 5122-5174.

Chaturvedi, R. K., J. Joshi, M. Jayaraman, G. Bala, and N. H. Ravindranath, 2012: Multimodel climate change projections for India under Representative Concentration Pathways (RCPs): A preliminary analysis. Curr. Sci., 103, 791-802.

Chauvin, F., and J.-F. Royer, 2010: Role of the SST Anomaly structures in response of cyclogenesis to global warming. In: Hurricanes and Climate Change [J. B. Elsner, R. E. Hodges, J. C. Malmstadt and K. N. Scheitlin (eds.)]. Springer Science+Business Media, Dordrecht, Netherlands, pp. 39-56.

Chen, D., 2003: A comparison of wind products in the context of ENSO prediction. Geophys. Res. Lett., 30, doi: 10.1029/2002GL016121.

Chen, G., I. M. Held, and W. A. Robinson, 2007: Sensitivity of the latitude of the surface westerlies to surface friction. J. Atmos. Sci., 64, 2899-2915.

Chen, G., J. Lu, and D. M. W. Frierson, 2008: Phase speed spectra and the latitude of surface westerlies: Interannual variability and global warming trend. J. Clim. 21, 5942-5959.

Chen, T.-C., and J.-h. Yoon, 2002: Interdecadal variation of the North Pacific wintertime blocking. Mon. Weather Rev., 130, 3136-3143.

Chen, W., Z. Jiang, L. Li, and P. Yiou, 2011: Simulation of regional climate change under the IPCC A2 scenario in southeast China. Clim. Dyn., 36, 491-507.

Cherchi, A., and A. Navarra, 2007: Sensitivity of the Asian summer monsoon to the horizontal resolution: Differences between AMIP-type and coupled model experiments. Clim. Dyn., 28, 273-290.

Cheung, H. N., W. Zhou, H. Y. Mok, and M. C. Wu, 2012: Relationship between UralSiberian blocking and the East Asian winter monsoon in relation to the Arctic Oscillation and the El Niño-Southern Oscillation. J. Clim., 25, 4242-4257.

Chiang, J., and D. Vimont, 2004: Analogous Pacific and Atlantic meridional modes of tropical atmosphere-ocean variability. J. Clim., 4143-4158.

Choi, D. H., J. S. Kug, W. T. Kwon, F. F. Jin, H. J. Baek, and S. K. Min, 2010: Arctic Oscillation responses to greenhouse warming and role of synoptic eddy feedback. J. Geophys. Res. Atmos., 115, doi: 10.1029/2010jd014160.

Choi, J., S. An, and S. Yeh, 2012: Decadal amplitude modulation of two types of ENSO and its relationship with the mean state. Clim. Dyn., 38, 2631-2644.

Choi, J., S. I. An, B. Dewitte, and W. W. Hsieh, 2009: Interactive feedback between the Tropical Pacific Decadal Oscillation and ENSO in a Coupled General Circulation Model. J. Clim., 22, 6597-6611.

Choi, J., S.-I. An, J.-S. Kug, and S.-W. Yeh, 2011: The role of mean state on changes in El Niño's flavor. Clim. Dyn., 37, 1205-1215.

Chotamonsak, C., E. P. Salathe, Jr., J. Kreasuwan, S. Chantara, and K. Siriwitayakorn, 2011: Projected climate change over Southeast Asia simulated using a WRF regional climate model. Atmos. Sci. Lett., 12, 213-219.

Chou, C., J. D. Neelin, U. Lohmann, and J. Feichter, 2005: Local and remote impacts of aerosol climate forcing on tropical precipitation. J. Clim., 18, 4621-4636.

Chou, C., J. C. H. Chiang, C.-W. Lan, C.-H. Chung, Y.-C. Liao, and C.-J. Lee, 2013: Increase in the range between wet and dry season precipitation. Nature Geosci. 6, 263-267.

Chou, S., et al., 2012: Downscaling of South America present climate driven by 4-member HadCM3 runs. Clim. Dyn., 38, 635-653.

Christensen, J. H., et al., 2007: Regional climate projections. In: Climate Change 2007: The Physical Science Basis. Contribution of Working Group I to the Fourth Assessment Report of the Intergovernmental Panel on Climate Change [Solomon, S., D. Qin, M. Manning, Z. Chen, M. Marquis, K. B. Averyt, M. Tignor and H. L. Miller (eds.)] Cambridge University Press, Cambridge, United Kingdom and New York, NY, USA, pp. 847-940.

Christiansen, B., 2005: The shortcomings of nonlinear principal component analysis in identifying circulation regimes. J. Clim., 18, 4814-4823.

Chung, C. E., and V. Ramanathan, 2006: Weakening of North Indian SST gradients and the monsoon rainfall in India and the Sahel. J. Clim., 19, 2036-2045.

Chung, C. E., and V. Ramanathan, 2007: Relationship between trends in land precipitation and tropical SST gradient. Geophys. Res. Lett., 34, doi: 10.1029/2007gl030491.

Chylek, P., and G. Lesins, 2008: Multidecadal variability of Atlantic hurricane activity: 1851-2007. J. Geophys. Res., 113, D22106. 
Chylek, P., C. K. Folland, G. Lesins, and M. Dubey, 2010: The 20th Century bipolar seesaw of the Arctic and Antarctic surface air temperatures. Geophys. Res. Lett., 37, doi: 10.1029/2010GL042793.

Chylek, P., C. K. Folland, G. Lesins, M. Dubey, and M. Wang, 2009: Arctic air temperature change amplification and the Atlantic Multidecadal Oscillation. Geophys. Res. Lett., 36, doi: 10.1029/ 2009GL038777.

Chylek, P., C. Folland, L. Frankcombe, H. Dijkstra, G. Lesins, and M. Dubey, 2012: Greenland ice core evidence for spatial and temporal variability of the Atlantic Multidecadal Oscillation. Geophys. Res. Lett., 39, L09705.

Cobb, K. M., C. D. Charles, H. Cheng, and R. L. Edwards, 2003: El Nino/Southern Oscillation and tropical Pacific climate during the last millennium. Nature, 424, 271-276.

Coelho, C. A. S., and L. Goddard, 2009: El Nino-induced tropical droughts in climate change projections. J. Clim., 22, 6456-6476.

Colle, B. A., Z. Zhang, K. A. Lombardo, E. Chang, P. Liu, and M. Zhang, 2013: Historical evaluation and future prediction of eastern North America and western Atlantic extratropical cyclones in the CMIP5 models during the cool season. J. Clim., 26, 6882-6903.

Collins, M., et al., 2010: The impact of global warming on the tropical Pacific ocean and El Niño. Nature Geosci., 3, 391-397.

Colman, R. A., A. F. Moise, and L. I. Hanson, 2011: Tropical Australian climate and the Australian monsoon as simulated by 23 CMIP3 models. J. Geophys. Res. Atmos., 116, doi: 10.1029/2010jd015149.

Comarazamy, D. E., and J. E. Gonzalez, 2011: Regional long-term climate change (1950-2000) in the midtropical Atlantic and its impacts on the hydrological cycle of Puerto Rico. J. Geophys. Res. Atmos., 116, doi: 10.1029/2010jd015414.

Compo, G. P., and P. D. Sardeshmukh, 2010: Removing ENSO-related variations from the climate record. J. Clim., 23, 1957-1978.

Conway, D., C. Hanson, R. Doherty, and A. Persechino, 2007: GCM simulations of the Indian Ocean dipole influence on East African rainfall: Present and future. Geophys. Res. Lett., 34, doi: 10.1029/2006GL027597.

Cook, B., N. Zeng, and J.-H. Yoon, 2011: Will Amazonia dry out? Magnitude and causes of change from IPCC Climate Model Projections. Earth Interact., 16, $1-27$.

Cook, B. I., and R. Seager, 2013: The response of the North American Monsoon to increased greenhouse gas forcing. J. Geophys. Res., 118,

Cook, K., 2008: Climate science: The mysteries of Sahel droughts. Nature Geosci., $1,647-648$.

Cook, K. H., and E. K. Vizy, 2006: Coupled model simulations of the west African monsoon system: Twentieth- and twenty-first-century simulations. J. Clim., 19, 3681-3703.

Cook, K. H., and E. K. Vizy, 2010: Hydrodynamics of the Caribbean Low-Level Jet and its relationship to precipitation. J. Clim., 23, 1477-1494.

Coppola, E., F. Kucharski, F. Giorgi, and F. Molteni, 2005: Bimodality of the North Atlantic Oscillation in simulations with greenhouse gas forcing. Geophys. Res. Lett., 32, doi: 10.1029/2005gl024080.

Cravatte, S., T. Delcroix, D. Zhang, M. McPhaden, and J. Leloup, 2009: Observed freshening and warming of the western Pacific Warm Pool. Clim. Dyn., 33, 565-589.

Croci-Maspoli, M., C. Schwierz, and H. Davies, 2007a: Atmospheric blocking: Spacetime links to the NAO and PNA. Clim. Dyn., 29, 713-725.

Croci-Maspoli, M., C. Schwierz, and H. C. Davies, 2007b: A multifaceted climatology of atmospheric blocking and its recent linear trend. J. Clim., 20, 633-649.

Cunningham, C. A. C., and I. F. D. Cavalcanti, 2006: Intraseasonal modes of variability affecting the South Atlantic Convergence Zone. Int. J. Climatol., 26, 1165-1180.

d'Orgeval, T., J. Polcher, and L. Li, 2006: Uncertainties in modelling future hydrological change over West Africa. Clim. Dyn., 26, 93-108.

Dacre, H. F., and S. L. Gray, 2009: The spatial distribution and evolution characteristics of North Atlantic Cyclones. Mon. Weather Rev., 137, 99-115.

Dai, A., 2011: Drought under global warming: A review. WIREs Clim. Change, 2, $45-65$.

Dai, A., 2013: Increasing drought under global warming in observations and models. Nature Clim. Change, 3, 52-58.

Dai, A., T. Qian, K. E. Trenberth, and J. D. Milliman, 2009: Changes in continental freshwater discharge from 1948 to 2004. J. Clim., 22, 2773-2792.

Dairaku, K., S. Emori, and T. Nozawa, 2008: Impacts of global warming on hydrological cycles in the Asian monsoon region. Adv. Atmos. Sci., 25, 960-973.

Dash, S. K., M. A. Kulkarni, U. C. Mohanty, and K. Prasad, 2009: Changes in the characteristics of rain events in India. J. Geophys. Res. Atmos., 114, D10109.
Davini, P., C. Cagnazzo, S. Gualdi, and A. Navarra, 2012: Bidimensional diagnostics, variability, and trends of Northern Hemisphere blocking. J. Clim., 25, 6496-6509.

Dawson, A., T. N. Palmer, and S. Corti, 2012: Simulating regime structures in weather and climate prediction models. Geophys. Res. Lett., 39, L21805.

de Oliveira Vieira, S., P. Satyamurty, and R. V. Andreoli, 2013: On the South Atlantic Convergence Zone affecting southern Amazonia in austral summer. Atmos. Sci. Lett., 14, 1-6.

de Vries, H., T. Woollings, J. Anstey, R. J. Haarsma, and W. Hazeleger, 2013: Atmospheric blocking and its relation to jet changes in a future climate. Clim. Dyn., doi:10.1007/s00382-013-1699-7, 1-12.

Dean, S., and P. Stott, 2009: The effect of local circulation variability on the detection and attribution of New Zealand temperature trends. J. Clim., 22, 6217-6229.

DeFries, R., L. Bounoua, and G. Collatz, 2002: Human modification of the landscape and surface climate in the next fifty years. Global Change Biol., 8, 438-458.

Della-Marta, P. M., and J. G. Pinto, 2009: Statistical uncertainty of changes in winter storms over the North Atlantic and Europe in an ensemble of transient climate simulations. Geophys. Res. Lett., 36, doi: 10.1029/2009gl038557.

Deni, S. M., J. Suhaila, W. Z. W. Zin, and A. A. Jemain, 2010: Spatial trends of dry spells over Peninsular Malaysia during monsoon seasons. Theor. Appl. Climatol., 99, 357-371.

Déqué, M., S. Somot, E. Sanchez-Gomez, C. M. Goodess, D. Jacob, G. Lenderink, and O. B. Christensen, 2012: The spread amongst ENSEMBLES regional scenarios: Regional climate models, driving general circulation models and interannual variability. Clim. Dyn., 38, 951-964.

Deser, C., A. S. Phillips, and M. A. Alexander, 2010a: Twentieth century tropical sea surface temperature trends revisited. Geophys. Res. Lett., 37, doi: 10.1029/2010gl043321.

Deser, C., M. A. Alexander, S.-P. Xie, and A. S. Phillips, 2010b: Sea surface temperature variability: Patterns and mechanisms. Annu. Rev. Mar. Sci., 2, 115-143.

Deser, C., R. Tomas, M. Alexander, and D. Lawrence, 2010c: The seasonal atmospheric response to projected Arctic sea ice loss in the late twenty-first century. J. Clim., 23, 333-351.

Deser, C., A. Phillips, V. Burdette, and H. Teng, 2012: Uncertainty in climate change projections: The role of internal variability. Clim. Dyn., 38, 527-546.

Di Lorenzo, E., et al., 2009: Nutrient and salinity decadal variations in the central and eastern North Pacific. Geophys. Res. Lett., 36, doi: 10.1029/2009GL038261.

Diamond, H. J., A. M. Lorrey, and J. A. Renwick, 2012: A southwest Pacific tropical cyclone climatology and linkages to the El Niño-Southern Oscillation. J. Clim., 26, 3-25.

Diffenbaugh, N. S., and M. Ashfaq, 2010: Intensification of hot extremes in the United States. Geophys. Res. Lett., 37, L15701.

Diffenbaugh, N. S., M. Scherer, and M. Ashfaq, 2013: Response of snow-dependent hydrologic extremes to continued global warming. Nature Clim. Change, 3, 379-384.

DiNezio, P. N., A. C. Clement, G. A. Vecchi, B. J. Soden, and B. P. Kirtman, 2009: Climate response of the equatorial Pacific to global warming. J. Clim., 22, 4873-4892.

Ding, Q., E. Steig, D. Battisti, and M. Kuttel, 2011: Winter warming in West Antarctica caused by central tropical Pacific warming. Nature Geosci., 4, 398-403.

Ding, Y., and J. C. L. Chan, 2005: The East Asian summer monsoon: An overview. Meteorol. Atmos. Phys., 89, 117-142.

Ding, Y., G. Ren, Z. Zhao, Y. Xu, Y. Luo, Q. Li, and J. Zhang, 2007: Detection, causes and projection of climate change over China: An overview of recent progress. Adv. Atmos. Sci, doi:DOI 10.1007/s00376-007-0954-4, 954-971.

Dole, R., M. Hoerling, J. Perlwitz, J. Eischeid, and P. Pegion, 2011: Was there a basis for anticipating the 2010 Russian heat wave? Geophys. Res. Lett., L06702, doi 10.1029/2010GL046582.

Dominguez, F., E. Rivera, D. P. Lettenmaier, and C. L. Castro, 2012: Changes in winter precipitation extremes for the western United States under a warmer climate as simulated by regional climate models. Geophys. Res. Lett., 39, L05803.

Donat, M. G., G. C. Leckebusch, S. Wild, and U. Ulbrich, 2011: Future changes in European winter storm losses and extreme wind speeds inferred from GCM and RCM multi-model simulations. Nat. Hazards Earth Syst. Sci., 11, 1351-1370.

Dong, B., R. T. Sutton, and T. Woollings, 2011: Changes of interannual NAO variability in response to greenhouse gases forcing. Clim. Dyn., 37, 1621-1641.

Dong, L., T. J. Vogelsang, and S. J. Colucci, 2008: Interdecadal trend and ENSO-related interannual variability in Southern Hemisphere blocking. J. Clim., 21, 3068-3077.

Döscher, R., and T. Koenigk, 2012: Arctic rapid sea ice loss events in regional coupled climate scenario experiments. Ocean Sci. Discuss., 9, 2327-2373. 
Dowdy, A. J., G. A. Mills, B. Timbal, and Y. Wang, 2012: Changes in the risk of extratropical cyclones in eastern Australia. J. Clim., 26, 1403-1417.

Drumond, A. R. M., and T. Ambrizzi, 2005: The role of SST on the South American atmospheric circulation during January, February and March 2001. Clim. Dyn., 24, 781-791.

Du, Y., and S.-P. Xie, 2008: Role of atmospheric adjustments in the tropical Indian Ocean warming during the 20th century in climate models. Geophys. Res. Lett., 35, doi: 10.1029/2008GL033631.

Du, Y., L. Yang, and S. Xie, 2011: Tropical Indian Ocean influence on Northwest Pacific tropical cyclones in summer following strong El Niño. J. Clim., 24, 315-322.

$\mathrm{Du}$, Y., S. P. Xie, G. Huang, and K. M. Hu, 2009: Role of air-sea interaction in the long persistence of El Niño-induced north Indian Ocean warming. J. Clim., 22, 2023-2038.

Dufek, A. S., T. Ambrizzi, and R. P. Rocha, 2008: Are reanalysis data useful for calculating climate indices over South America? Ann. NY Acad. Sci, 1146, 87-104.

Duffy, P. B., and C. Tebaldi, 2012: Increasing prevalence of extreme summer temperatures in the U.S. Clim. Change, 111, 487-495.

Dunion, J., and C. Velden, 2004: The impact of the Saharan air layer on Atlantic tropical cyclone activity. Bull. Am. Meteorol. Soc., 85, 353-365.

Dunion, J., and C. Marron, 2008: A reexamination of the Jordan mean tropical sounding based on awareness of the Saharan air layer: Results from 2002. J. Clim., 21, 5242-5253.

Dunion, J. P., 2011: Rewriting the climatology of the tropical North Atlantic and Caribbean Sea atmosphere. J. Clim., 24, 893-908.

Dunn-Sigouin, E., and S.-W. Son, 2013: Northern Hemisphere blocking frequency and duration in the CMIP5 models. J. Geophys. Res. Atmos., 118, 1179-1188.

Elsner, J. B., J. P. Kossin, and T. H. Jagger, 2008: The increasing intensity of the strongest tropical cyclones. Nature, 455, 92-95.

Emanuel, K., 2007: Environmental factors affecting tropical cyclone power dissipation. J. Clim., 20, 5497-5509.

Emanuel, K., 2010: Tropical cyclone activity downscaled from NOAA-CIRES reanalysis, 1908-1958. J. Adv. Model. Earth Syst., 2, 12.

Emanuel, K., R. Sundararajan, and J. Williams, 2008: Hurricanes and global warming: Results from downscaling IPCC AR4 simulations. Bull. Am. Meteorol. Soc., 89, 347-367.

Emanuel, K., S. Solomon, D. Folini, S. Davis, and C. Cagnazzo, 2012: Influence of tropical tropopause layer cooling on Atlantic hurricane activity. J. Clim., 26, 2288-2301.

Endo, H., 2010: Long-term changes of seasonal progress in Baiu rainfall using 109 years (1901-2009) daily station data. Sola, 7, 5-8.

Endo, H., 2012: Future changes of Yamase bringing unusually cold summers over northeastern Japan in CMIP3 multi-models. J. Meteorol. Soc. Jpn., 90A, 123-136.

Endo, H., A. Kitoh, T. Ose, R. Mizuta, and S. Kusunoki, 2012: Future changes and uncertainties in Asian precipitation simulated by multiphysics and multi-sea surface temperature ensemble experiments with high-resolution Meteorological Research Institute atmospheric general circulation models (MRI-AGCMs). J. Geophys. Res., 117, D16118.

Enfield, D., S. K. Lee, and C. Wang, 2006: How are large Western Hemisphere warm pools formed? Prog. Oceanogr., 70, 346-365.

Engelbrecht, C. J., F. A. Engelbrecht, and L. L. Dyson, 2011: High-resolution modelprojected changes in mid-tropospheric closed-lows and extreme rainfall events over southern Africa. Int. J. Climatol., 33, 173-187.

England, M. H., C. C. Ummenhofer, and A. Santoso, 2006: Interannual rainfall extremes over southwest Western Australia linked to Indian ocean climate variability. J. Clim., 19, 1948-1969.

Englehart, P. J., and A. V. Douglas, 2006: Defining intraseasonal rainfall variability within the North American monsoon. J. Clim., 19, 4243-4253.

Evan, A., 2012: Atlantic hurricane activity following two major volcanic eruptions. J. Geophys. Res. Atmos., 117, doi: 10.1029/2011JD016716.

Evan, A., G. Foltz, D. Zhang, and D. Vimont, 2011a: Influence of African dust on oceanatmosphere variability in the tropical Atlantic. Nature Geosci., 4, 762-765.

Evan, A. T., J. P. Kossin, C. E. Chung, and V. Ramanathan, 2011 b: Arabian Sea tropical cyclones intensified by emissions of black carbon and other aerosols. Nature, 479, 94-97.

Evan, A. T., J. P. Kossin, C. Chung, and V. Ramanathan, 2012: Evan et al. reply to Wang et al. (2012), "Intensified Arabian Sea tropical storms". Nature, 489, E2-E3.
Evan, A. T., D. J. Vimont, A. K. Heidinger, J. P. Kossin, and R. Bennartz, 2009: The role of aerosols in the evolution of tropical North Atlantic Ocean temperature anomalies. Science, 324, 778-781.

Evans, J. P., 2008: Changes in water vapor transport and the production of precipitation in the eastern Fertile Crescent as a result of global warming. J. Hydrometeorol., 9, 1390-1401.

Evans, J. P., 2009: 21st century climate change in the Middle East. Clim. Change, $92,417-432$.

Eyring, V., et al., 2013: Long-term ozone changes and associated climate impacts in CMIP5 simulations. J. Geophys. Res. Atmos, doi:10.1002/jgrd.50316.

Falvey, M., and R. D. Garreaud, 2009: Regional cooling in a warming world: Recent temperature trends in the southeast Pacific and along the west coast of subtropical South America (1979-2006). J. Geophys. Res. Atmos., 114, D04102.

Fauchereau, N., B. Pohl, C. Reason, M. Rouault, and Y. Richard, 2009: Recurrent daily OLR patterns in the Southern Africa/Southwest Indian Ocean region, implications for South African rainfall and teleconnections. Clim. Dyn., 32, 575-591.

Fedorov, A. V., and S. G. Philander, 2000: Is El Nino changing? Science, 288, 19972002.

Feldstein, S. B., and C. Franzke, 2006: Are the North Atlantic Oscillation and the Northern Annular Mode distinguishable? J. Atmos. Sci., 63, 2915-2930.

Feliks, Y., M. Ghil, and A. W. Robertson, 2010: Oscillatory climate modes in the eastern Mediterranean and their synchronization with the North Atlantic Oscillation. J. Clim., 23, 4060-4079.

Feng, S., Q. Hu, and R. Oglesby, 2011: Influence of Atlantic sea surface temperatures on persistent drought in North America. Clim. Dyn., 37, 569-586.

Fereday, D. R., J. R. Knight, A. A. Scaife, C. K. Folland, and A. Philipp, 2008: Cluster analysis of North Atlantic-European circulation types and links with tropical Pacific sea surface temperatures. J. Clim., 21, 3687-3703.

Fink, A., S. Pohle, J. Pinto, and P. Knippertz, 2012: Diagnosing the influence of diabatic processes on the explosive deepening of extratropical cyclones. Geophys. Res. Lett., 39, doi: 10.1029/2012GL051025.

Fink, A. H., T. Bruecher, V. Ermert, A. Krueger, and J. G. Pinto, 2009: The European storm Kyrill in January 2007: Synoptic evolution, meteorological impacts and some considerations with respect to climate change. Nat. Hazards Earth Syst. Sci., 9, 405-423.

Fischer-Bruns, I., D. F. Banse, and J. Feichter, 2009: Future impact of anthropogenic sulfate aerosol on North Atlantic climate. Clim. Dyn., 32, 511-524.

Fogt, R., D. Bromwich, and K. Hines, 2011: Understanding the SAM influence on the South Pacific ENSO teleconnection. Clim. Dyn., 36, 1555-1576.

Fogt, R. L., J. Perlwitz, A. J. Monaghan, D. H. Bromwich, J. M. Jones, and G. J. Marshall, 2009: Historical SAM variability. Part II: Twentieth-century variability and Ttrends from reconstructions, observations, and the IPCC AR4 models. J. Clim., 22, 53465365.

Folland, C., M. Salinger, N. Jiang, and N. Rayner, 2003: Trends and variations in South Pacific island and ocean surface temperatures. J. Clim., 16, 2859-2874.

Folland, C. K., J. A. Renwick, M. J. Salinger, and A. B. Mullan, 2002: Relative influences of the Interdecadal Pacific Oscillation and ENSO on the South Pacific Convergence Zone. Geophys. Res. Lett., 29, doi: 10.1029/2001GL014201.

Folland, C. K., J. Knight, H. W. Linderholm, D. Fereday, S. Ineson, and J. W. Hurrell, 2009: The summer North Atlantic Oscillation: Past, present, and future. J. Clim., 22, 1082-1103.

Forster, P., et al., 2007: Changes in atmospheric constituents and in radiative forcing In: Climate Change 2007: The Physical Science Basis. Contribution of Working Group I to the Fourth Assessment Report of the Intergovernmental Panel on Climate Change [Solomon, S., D. Qin, M. Manning, Z. Chen, M. Marquis, K. B. Averyt, M. Tignor and H. L. Miller (eds.)] Cambridge University Press, Cambridge, United Kingdom and New York, NY, USA, pp. 129-234.

Frank, W., and P. Roundy, 2006: The role of tropical waves in tropical cyclogenesis. Mon. Weather Rev., 134, 2397-2417.

Frederiksen, C. S., J. S. Frederiksen, J. M. Sisson, and S. L. Osbrough, 2011a: Changes and projections in the annual cycle of the Southern Hemisphere circulation, storm tracks and Australian rainfall. Int. J. Clim. Change Impacts Respons., 2, 143-162.

Frederiksen, C. S., J. S. Frederiksen, J. M. Sisson, and S. L. Osbrough, 2011 b: Australian winter circulation and rainfall changes and projections. Int. J. Clim. Change Strat. Manage., 3, 170-188.

Frederiksen, J. S., and C. S. Frederiksen, 2007: Interdecadal changes in southern hemisphere winter storm track modes. Tellus A, 59, 599-617. 
Frederiksen, J. S., C. S. Frederiksen, S. L. Osbrough, and J. M. Sisson, 2010: Causes of changing Southern Hemispheric weather systems. In: Managing Climate Change [I. Jupp, P. Holper and W. Cai (eds.)]. CSIRO Publishing, Collingwood, Victoria, Australia, pp. 85-98.

Friedman, A. R., Y. T. Hwang, J. C. H. Chiang, and D. M. W. Frierson, 2013: Interhemispheric temperature asymmetry over the 20th century and in future projections. J. Clim., doi:10.1175/JCLI-D-12-00525.1.

Frierson, D. M. W., I. M. Held, and P. Zurita-Gotor, 2007: A gray-radiation aquaplanet moist GCM. Part II: Energy transports in altered climates. J. Atmos. Sci., 64, 1680-1693.

Fučkar, N. S., S.-P. Xie, R. Farneti, E. A. Maroon, and D. M. W. Frierson, 2013: Influence of the extratropical ocean circulation on the intertropical convergence zone in an idealized coupled general circulation model. J. Clim., 26, 4612-4629.

Furtado, J., E. Di Lorenzo, N. Schneider, and N. A. Bond, 2011: North Pacific decadal variability and climate change in the IPCC AR4 models. J. Clim., 24, 3049-3066.

Gamble, D. W., and S. Curtis, 2008: Caribbean precipitation: Review, model and prospect. Prog. Phys. Geogr., 32, 265-276.

Gan, M. A., V. B. Rao, and M. C. L. Moscati, 2006: South American monsoon indices. Atmos. Sci. Lett., 6, 219-223.

Gao, X., Y. Shi, and F. Giorgi, 2012a: A high resolution simulation of climate change over China. Sci. China Earth Sci., 54, 462-472.

Gao, X., Y. Shi, R. Song, F. Giorgi, Y. Wang, and D. Zhang, 2008: Reduction of future monsoon precipitation over China: Comparison between a high resolution RCM simulation and the driving GCM. Meteorol. Atmos. Phys., 100, 73-86.

Gao, X., Y. Shi, D. Zhang, J. Wu, F. Giorgi, Z. Ji, and Y. Wang, 2012b: Uncertainties in monsoon precipitation projections over China: Results from two high-resolution RCM simulations. Clim. Res., 2, 213.

Gao, Y., L. R. Leung, E. P. Salathé, F. Dominguez, B. Nijssen, and D. P. Lettenmaier, 2012c: Moisture flux convergence in regional and global climate models: Implications for droughts in the southwestern United States under climate change. Geophys. Res. Lett., 39, L09711.

Garcia, R., and W. J. Randel, 2008: Acceleration of the Brewer-Dobson circulation due to increases in greenhouse gases. J. Atmos. Sci., 65, 2731-2739.

Garfinkel, C. I., and D. L. Hartmann, 2011: The influence of the Quasi-Biennial Oscillation on the troposphere in wintertime in a hierarchy of models, Part 1: Simplified dry GCMs. J. Atmos. Sci., 68, 1273-1289.

Gastineau, G., and B. J. Soden, 2009: Model projected changes of extreme wind events in response to global warming. Geophys. Res. Lett., 36, doi: $10.1029 / 2009$ gl037500.

Geng, Q. Z., and M. Sugi, 2003: Possible change of extratropical cyclone activity due to enhanced greenhouse gases and sulfate aerosols - Study with a highresolution AGCM. J. Clim., 16, 2262-2274.

Gerber, E. P., and G. K. Vallis, 2007: Eddy-zonal flow interactions and the persistence of the zonal index. J. Atmos. Sci., 64, 3296-3311.

Gerber, E. P., L. M. Polvani, and D. Ancukiewicz, 2008: Annular mode time scales in the Intergovernmental Panel on Climate Change Fourth Assessment Report models. Geophys. Res. Lett., 35, doi: 10.1029/2008gl035712.

Gerber, E. P., et al., 2010: Stratosphere-troposphere coupling and annular mode variability in chemistry-climate models. J. Geophys. Res., 115, doi: 10.1029/2009jd013770.

Giannini, A., R. Saravanan, and P. Chang, 2003: Oceanic forcing of Sahel rainfall on interannual to interdecadal time scales. Science, 302, 1027-1030.

Giannini, A., M. Biasutti, I. Held, and A. Sobel, 2008: A global perspective on African climate. Clim. Change, 90, 359-383.

Giese, B., and S. Ray, 2011: El Niño variability in simple ocean data assimilation (SODA), 1871-2008. J. Geophys. Res. Oceans, 116, 10.1029/2010JC006695.

Gillett, N. P., and J. C. Fyfe, 2013: Annular mode changes in the CMIP5 simulations. Geophys. Res. Lett., 40,

Giorgi, F., and X. Bi, 2009: Time of emergence (TOE) of GHG-forced precipitation change hot-spots. Geophys. Res. Lett., 36, doi:10.1029/2009GL037593.

Gochis, D. J., L. Castillo-Brito, and J. Shuttleworth, 2007: Correlations between seasurface temperatures and warm season streamflow in northwest Mexico. Int. J. Climatol., 27, 883-901.

Goldenberg, S. B., C. Landsea, A. M. Mestas-Nunez, and W. M. Gray, 2001: The recent increase in Atlantic hurricane activity: Causes and implications. Science, 293, 474-479.

Gong, D. Y., and S. W. Wang, 1999: Definition of Antarctic Oscillation Index. Geophys. Res. Lett., 26, 459-462.
Gong, D. Y., and C. H. Ho, 2002: The Siberian High and climate change over middle to high latitude Asia. Theor. Appl. Climatol., 72, 1-9.

Good, P., J. A. Lowe, M. Collins, and W. Moufouma-Okia, 2008: An objective tropical Atlantic sea surface temperature gradient index for studies of south Amazon dry-season climate variability and change. Philos. Trans. R. Soc. London B, 363, $1761-1766$.

Goswami, B. N., V. Venugopal, D. Sengupta, M. S. Madhusoodanan, and P. K. Xavier, 2006: Increasing trend of extreme rain events over India in a warming environment. Science, 314, 1442-1445.

Graff, L., and J. LaCasce, 2012: Changes in the extratropical storm tracks in response to changes in SST in an AGCM. J. Clim., 25, 1854-1870.

Grantz, K., B. Rajagopalan, M. Clark, and E. Zagona, 2007: Seasonal shifts in the North American monsoon. J. Clim., 20, 1923-1935.

Griffiths, G., M. Salinger, and I. Leleu, 2003: Trends in extreme daily rainfall across the South Pacific and relationship to the South Pacific Convergence Zone. Int. J. Climatol., 23, 847-869.

Griffiths, G., et al., 2005: Change in mean temperature as a predictor of extreme temperature change in the Asia-Pacific region. Int. J. Climatol., 25, 1301-1330.

Griffiths, G. M., 2007: Changes in New Zealand daily rainfall extremes 1930 - 2004. Weather Clim., 27, 3-44.

Gu, D. F., and S. G. H. Philander, 1995: Secular changes of annual and interannual variability in the tropics during the past century. J. Clim., 8, 864-876.

Guilyardi, E., H. Bellenger, M. Collins, S. Ferrett, W. Cai, and A. Wittenberg, 2012: A first look at ENSO in CMIP5. CLIVAR Exchanges, 58, 29-32.

Guo, Z. C., D. H. Bromwich, and K. M. Hines, 2004: Modeled antarctic precipitation. Part II: ENSO modulation over West Antarctica. J. Clim., 17, 448-465.

Gutiérrez, D., et al., 2011: Coastal cooling and increased productivity in the main upwelling zone off Peru since the mid-twentieth century. Geophys. Res. Lett., 38, L07603.

Gutowski, W. J. et al., 2010: Regional, extreme monthly precipitation simulated by NARCCAP RCMs. J. Hydrometeorol., 11, 1373-1379.

Gutzler, D. S., 2004: An index of interannual precipitation variability in the core of the North American monsoon region. J. Clim., 17, 4473-4480.

Gutzler, D. S., and T. O. Robbins, 2011: Climate variability and projected change in the western United States: Regional downscaling and drought statistics. Clim. Dyn., 37, 835-849.

Gutzler, D. S., L. N. Long, J. Schemm, S. B. Roy, M. Bosilovich, J. C. Collier, M. Kanamitsu, P. Kelly, D. Lawrence, M. I. Lee, R. L. Sánchez, B. Mapes, K. Mo, A. Nunes, E. A. Ritchie, J. Roads, S. Schubert, H. Wei, and G. J. Zhang, 2009: Simulations of the 2004 North American Monsoon: NAMAP2. J. Climate, 22, 6716-6740.

Haarsma, R. J., et al., 2013: More hurricanes to hit Western Europe due to global warming. Geophys. Res. Lett., doi:10.1002/grl.50360.

Haensler, A., S. Hagemann, and D. Jacob, 2011: The role of the simulation setup in a long-term high-resolution climate change projection for the southern African region. Theor. Appl. Climatol., 106, 153-169.

Haerter, J., E. Roeckner, L. Tomassini, and J. von Storch, 2009: Parametric uncertainty effects on aerosol radiative forcing. Geophys. Res. Lett., 36, doi: 10.1029/2009GL039050.

Haigh, J. D., and H. K. Roscoe, 2006: Solar influences on polar modes of variability. Meteorol. Z., 15, 371-378.

Häkkinen, S., P. B. Rhines, and D. L. Worthen, 2011: Atmospheric blocking and atlantic multidecadal ocean variability. Science, 334, 655-659.

Hall, T., A. Sealy, T. Stephenson, S. Kusunoki, M. Taylor, A. A. Chen, and A. Kitoh, 2012: Future climate of the Caribbean from a super-high-resolution atmospheric general circulation model. Theor. Appl. Climatol., doi:10.1007/s00704-012-07797, 1-17.

Han, W., et al., 2010: Patterns of Indian Ocean sea-level change in a warming climate. Nature Geosci., 3, 546-550.

Handorf, K., and Dethloff, 2009: Atmospheric teleconnections and flow regimes under future climate projections. 237-255.

Hansen, J., M. Sato, R. Ruedy, K. Lo, D. W. Lea, and M. Medina-Elizade, 2006: Global temperature change. Proc. Natl. Acad. Sci. U.S.A., 103, 14288-14293.

Hansingo, K., and C. Reason, 2008: Modelling the atmospheric response to SST dipole patterns in the South Indian Ocean with a regional climate model. Meteorol. Atmos. Phys., 100, 37-52.

Hansingo, K., and C. Reason 2009: Modelling the atmospheric response over southern Africa to SST forcing in the southeast tropical Atlantic and southwest subtropical Indian Oceans. Int. J. Climatol., 29, 1001-1012. 
Harrison, S. P., et al., 2003: Mid-Holocene climates of the Americas: A dynamical response to changed seasonality. Clim. Dyn., 20, 663-688.

Hartmann, B., and G. Wendler, 2005: The Significance of the 1976 Pacific climate shift in the climatology of Alaska. J. Clim., 18, 4824-4839.

Harvey, B. J., L. C. Shaffrey, T. J. Woollings, G. Zappa, and K. I. Hodges, 2012: How large are projected 21st century storm track changes? Geophys. Res. Lett., 39, L18707.

Haylock, M. R., et al., 2006: Trends in total and extreme South American rainfall in 1960-2000 and links with sea surface temperature. J. Clim., 19, 1490-1512.

Held, I., and M. Zhao, 2011: The response of tropical cyclone statistics to an increase in $\mathrm{CO}_{2}$ with fixed sea surface temperatures. J. Clim., 24, 5353-5364.

Held, I., T. Delworth, J. Lu, K. Findell, and T. Knutson, 2005: Simulation of Sahel drought in the 20th and 21st centuries. Proc. Natl. Acad. Sci. U.S.A., 102, 17891-17896.

Held, I. M., 1993: Large-scale dynamics and global warming. Bull. Am. Meteorol. Soc., 74, 228-241.

Held, I. M., and B. J. Soden, 2006: Robust responses of the hydrological cycle to global warming. J. Clim., 19, 5686-5699.

Hendon, H. H., D. W. J. Thompson, and M. C. Wheeler, 2007: Australian rainfall and surface temperature variations associated with the Southern Hemisphere annular mode. J. Clim., 20, 2452-2467.

Hennessy, K., S. Power, and G. Cambers, Eds., 2011: Climate change in the Pacific: Scientific Assessment and New Research. Regional Overview (Volume 1) and Country Reports (Volume 2). Australian Bureau of Meteorology (BoM) and Commonwealth Scientific and Industrial Organisation (CSIRO), Melbourne, Australia.

Hermes, J., and C. Reason, 2009: Variability in sea-surface temperature and winds in the tropical south-east Atlantic Ocean and regional rainfall relationships. Int. J. Climatol., 29, 11-21.

Hernandez-Deckers, D., and J.-S. von Storch, 2010: Energetics responses to increases in greenhouse gas concentration. J. Clim., 23, 3874-3887.

Hidayat, R., and S. Kizu, 2010: Influence of the Madden-Julian Oscillation on Indonesian rainfall variability in austral summer. Int. J. Climatol., 30, 1816-1825.

Hill, K. J., A. S. Taschetto, and M. H. England, 2011: Sensitivity of South American summer rainfall to tropical Pacific Ocean SST anomalies. Geophys. Res. Lett., 38, L01701.

Hinton, T. J., B. J. Hoskins, and G. M. Martin, 2009: The influence of tropical sea surface temperatures and precipitation on North Pacific atmospheric blocking. Climate Dynamics, 33, 549-563.

Hirschi, M., et al., 2011: Observational evidence for soil-moisture impact on hot extremes in southeastern Europe. Nature Geosci., 4, 17-21.

Ho, C. K., D. B. Stephenson, M. Collins, C. A. T. Ferro, and S. J. Brown, 2012: Calibration strategies: A source of additional uncertainty in climate change projections. Bull. Am. Meteorol. Soc., 93, 21-26.

Hoerling, M., J. Hurrell, J. Eischeid, and A. Phillips, 2006: Detection and attribution of twentieth-century northern and southern African rainfall change. J. Clim., 19, 3989-4008.

Holland, G. J., and P. J. Webster, 2007: Heightened tropical cyclone activity in the North Atlantic: Natural variability or climate trend? Philos. Trans. R. Soc. London A, 365, 2695-2716.

Hope, P. K., W. Drosdowsky, and N. Nicholls, 2006: Shifts in the synoptic systems influencing southwest Western Australia. Clim. Dyn., 26, 751-764.

Horel, J. D., and J. M. Wallace, 1981: Planetary-scale atmospheric phenomena associated with the Southern Oscillation. Mon. Weather Rev., 109, 813-829.

Hori, M. E., D. Nohara, and H. L. Tanaka, 2007: Influence of Arctic Oscillation towards the Northern Hemisphere surface temperature variability under the global warming scenario. J. Meteorol. Soc. Jpn., 85, 847-859.

Hsieh, W. W., A. Wu, and A. Shabbar, 2006: Nonlinear atmospheric teleconnections. Geophys. Res. Lett., 33, doi: 10.1029/2005gl025471.

Hsu, P.-C., T. Li, and B. Wang, 2011: Trends in global monsoon area and precipitation in the past 30 years. Geophys. Res. Lett., 38, doi: 10.1029/2011GL046893.

Hsu, P.-C., T. Li, H. Murakami, and A. Kitoh, 2013: Future change of the global monsoon revealed from 19 CMIP5 models. J. Geophys. Res. Atmos., 118, doi: 10.1002/jgrd.50145.

Hu, Z., A. Kumar, B. Jha, and B. Huang, 2012a: An Analysis of Forced and internal variability in a warmer climate in CCSM3. J. Clim., 25, 2356-2373.

Hu, Z., A. Kumar, B. Jha, W. Wang, B. Huang, and B. Huang, 2012b: An analysis of warm pool and cold tongue El Niños: Air-sea coupling processes, global influences, and recent trends. Clim. Dyn., 38, 2017-2035.
Hu, Z. Z., 1997: Interdecadal variability of summer climate over East Asia and its association with $500 \mathrm{hPa}$ height and global sea surface temperature. J. Geophys. Res. Atmos., 102, 19403-19412.

Hu, Z.Z., and Z. H. Wu, 2004: The intensification and shift of the annual North Atlantic Oscillation in a global warming scenario simulation. Tellus $A, 56,112-124$.

Huang, B., and Z. Liu, 2001: Temperature trend of the last $40 \mathrm{yr}$ in the upper Pacific Ocean. J. Clim., 14, 3738-3750.

Huang, G., K. M. Hu, and S. P. Xie, 2010: Strengthening of tropical Indian Ocean teleconnection to the northwest Pacific since the mid-1970s: An atmospheric GCM study. J. Clim., 23, 5294-5304.

Huang, J., X. Guan, and F. Ji, 2012: Enhanced cold-season warming in semi-arid regions. Atmos. Chem. Phys. Discuss., 12, 4627-4653.

Huang, P., S.-P. Xie, K. Hu, G. Huang, and R. Huang, 2013: Patterns of the seasonal response of tropical rainfall to global warming. Nature Geosci., 6, 357-361.

Huang, R., W. Chen, B. Yang, and R. Zhang, 2004: Recent advances in studies of the interaction between the east Asian winter and summer monsoons and ENSO cycle. Adv. Atmos. Sci., 21, 407-424.

Huffman, G. J., R. F. Adler, D. T. Bolvin, and G. Gu, 2009: Improving the global precipitation record: GPCP Version 2.1. Geophys. Res. Lett., 36, L17808.

Hurrell, J. W., and C. Deser, 2009: North Atlantic climate variability: The role of the North Atlantic Oscillation. J. Mar. Syst., 78, 28-41.

Hurrell, J. W., Y. Kushnir, G. Ottersen, and M. Visbeck, 2003: An overview of the North Atlantic Oscillation. In: The North Atlantic Oscillation: Climate Significance and Environmental Impact [J. W. Hurrell, Y. Kushnir, M. Visbeck and G. Ottersen (eds.)]. American Geophysical Union, Washington, DC, pp. 1-35.

Huss, M., R. Hock, A. Bauder, and M. Funk, 2010: 100-year mass changes in the Swiss Alps linked to the Atlantic Multidecadal Oscillation. Geophys. Res. Lett., 37, doi: 10.1029/2010GL042616.

Hwang, Y.-T., and D. M. W. Frierson, 2010: Increasing atmospheric poleward energy transport with global warming. Geophys. Res. Lett., 37, doi: 10.1029/2010GL045440.

Ihara, C., Y. Kushnir, M. Cane, and V. de la Pena, 2009: Climate Change over the Equatorial Indo-Pacific in Global Warming. J. Clim., 22, 2678-2693.

lizumi, T., F. Uno, and M. Nishimori, 2012: Climate downscaling as a source of uncertainty in projecting local climate change impacts. J. Meteorol. Soc. Jpn., 90B, 83-90.

Im, E. S., J. B. Ahn, W. T. Kwon, and F. Giorgi, 2008: Multi-decadal scenario simulation over Korea using a one-way double-nested regional climate model system. Part 2: Future climate projection (2021-2050). Clim. Dyn., 30, 239-254.

Ineson, S., A. A. Scaife, J. R. Knight, J. C. Manners, N. J. Dunstone, L. J. Gray, and J. D. Haigh, 2011: Solar forcing of winter climate variability in the Northern Hemisphere. Nature Geosci., 4, 753-757.

Inoue, J., J. Liu, and J. A. Curry, 2006: Intercomparison of arctic regional climate models: Modeling clouds and radiation for SHEBA in May 1998. J. Clim., 19, 4167-4178.

Ionita, M., G. Lohmann, N. Rimbu, S. Chelcea, and M. Dima, 2012: Interannual to decadal summer drought variability over Europe and its relationship to global sea surface temperature. Clim. Dyn., 38, 363-377.

IPCC, 2007a: Climate Change 2007: The Physical Science Basis. Contribution of Working Group I to the Fourth Assessment Report of the Intergovernmental Panel on Climate Change [Solomon, S., D. Qin, M. Manning, Z. Chen, M. Marquis, K. B. Averyt, M. Tignor and H. L. Miller (eds.)] Cambridge University Press, Cambridge, United Kingdom and New York, NY, USA, 996 pp.

IPCC, 2007b: Climate Change 2007: Impacts, Adaptation and Vulnerability. Contribution of Working Group II to the Fourth Assessment Report of the Intergovernmental Panel on Climate Change (IPCC) [M. L. Parry, O. F. Canziani, J. P. Palutikof, P. J. van der Linden and C. E. Hanson (eds.)]. Cambridge University Press, Cambridge, United Kingdom and New York, NY, USA, 976 pp.

IPCC, 2012: Managing the Risks of Extreme Events and Disasters to Advance Climate Change Adaptation. A Special Report of Working Groups I and II of the Intergovernmental Panel on Climate Change [C. B. Field, V. Barros, T. F. Stocker, D. Qin, D. J. Dokken, K. L. Ebi, M. D. Mastrandrea, K. J. Mach, G.-K. Plattner, S. K. Allen, M. Tignor and P.M. Midgley (eds.)]. Cambridge University Press, Cambridge, United Kingdom and New York, NY, USA, 582 pp.

Irving, D., P. Whetton, and A. Moise, 2012: Climate projections for Australia: A first glance at CMIP5. Aust. Mereorol. Oceanogr. J., 62, 211-225.

Irving, D., et al., 2011: Evaluating global climate models for the Pacific island region. Clim. Res., 49, 169-187. 
Izumo, T., C. D. Montegut, J. J. Luo, S. K. Behera, S. Masson, and T. Yamagata, 2008: The role of the western Arabian Sea upwelling in Indian monsoon rainfall variability. J. Clim., 21, 5603-5623.

Jaeger, E. B., and S. I. Seneviratne, 2010: Impact of soil moisture-atmosphere coupling on European climate extremes and trends in a regional climate model. Clim. Dyn., 36, 1919-1939.

Janicot, S., et al., 2011: Intraseasonal variability of the West African monsoon. Atmos. Sci. Lett., 12, 58-66.

Jiang, H., and E. Zipser, 2010: Contribution of tropical cyclones to the global precipitation from eight seasons of TRMM data: Regional, seasonal, and interannual variations. J. Clim., 23, 1526-1543.

Jiang, Y. L., and Z. Zhao, 2013: Maximum wind speed changes over China. Acta Meteorol. Sin., 27, 63-74.

Jiang, Z., J. Song, L. Li, W. Chen, Z. Wang, and J. Wang, 2011: Extreme climate events in China: IPCC-AR4 model evaluation and projection. Clim. Change, 110, 385401.

Jin, F., A. Kitoh, and P. Alpert, 2010: Water cycle changes over the Mediterranean: A comparison study of a super-high-resolution global model with CMIP3. Philos. Trans. R. Soc. London A, 68, 5137-5149.

Johanson, C. M., and Q. Fu, 2009: Hadley cell widening: Model simulations versus observations. J. Clim., 22, 2713-2725.

Joly, M., A. Voldoire, H. Douville, P. Terray, and J.-F. Royer, 2007: African monsoon teleconnections with tropical SSTs: Validation and evolution in a set of IPCC4 simulations. Clim. Dyn., 29, 1-20.

Jones, C., and L. M. V. Carvalho, 2013: Climate change in the South American Monsoon System: Present climate and CMIP5 projections. J. Clim., doi:10.1175/ JCLI-D-12-00412.1.

Jones, D. A., W. Wang, and R. Fawcett, 2009a: High-quality spatial climate data-sets for Australia. Aust. Meteorol. Oceanogr. J., 58, 233-248.

Jones, J. M., R. L. Fogt, M. Widmann, G. J. Marshall, P. D. Jones, and M. Visbeck, 2009b: Historical SAM variability. Part l: Century-ength seasonal reconstructions. J. Clim., 22, 5319-5345.

Joshi, M. M., A. J. Charlton, and A. A. Scaife, 2006: On the influence of stratospheric water vapor changes on the tropospheric circulation. Geophys. Res. Lett., 33, doi: $10.1029 / 2006 \mathrm{gl} 025983$.

Jourdain, N., A. Gupta, A. Taschetto, C. Ummenhofer, A. Moise, and K. Ashok, 2013: The Indo-Australian monsoon and its relationship to ENSO and IOD in reanalysis data and the CMIP3/CMIP5 simulations. Clim. Dyn., doi:10.1007/s00382-0131676-1, 1-30.

Jung, T., et al., 2011: High-resolution global climate simulations with the ECMWF model in project Athena: Experimental design, model climate, and seasonal forecast skill. J. Clim., 25, 3155-3172.

Junquas, C., C. Vera, L. Li, and H. Treut, 2012: Summer precipitation variability over Southeastern South America in a global warming scenario. Climate Dynamics, $38,1867-1883$.

Junquas, C., C. S. Vera, L. Li, and H. Treut, 2013: Impact of projected SST changes on summer rainfall in southeastern South America. Clim. Dyn., 40, 1569-1589.

Kajikawa, Y., B. Wang, and J. Yang, 2010: A multi-time scale Australian monsoon index. Int. J. Climatol., 30, 1114-1120.

Kanada, S., M. Nakano, and T. Kato, 2010: Changes in mean atmospheric structures around Japan during July due to global warming in regional climate experiments using a cloud resolving model. Hydrol. Res. Lett., 4, 11-14.

Kanada, S., M. Nakano, and T. Kato, 2012: Projections of future changes in precipitation and the vertical structure of the frontal zone during the Baiu season in the vicinity of Japan using a 5-km-mesh regional climate model. J. Meteorol. Soc. Jpn., 90A, 65-86.

Kang, S., I. Held, D. Frierson, and M. Zhao, 2008: The response of the ITCZ to extratropical thermal forcing: Idealized slab-ocean experiments with a GCM. J. Clim., 21, 3521-3532.

Kao, H. Y., and J.Y. Yu, 2009: Contrasting Eastern-Pacific and Central-Pacific types of ENSO. J. Clim., 22, 615-632.

Kapnick, S., and A. Hall, 2012: Causes of recent changes in western North American snowpack. Clim. Dyn., 38, 1885-1899.

Karmalkar, A. V., R. S. Bradley, and H. F. Diaz, 2011: Climate change in Central America and Mexico: Regional climate model validation and climate change projections. Clim. Dyn., 37, 605-629.

Karnauskas, K. B., R. Seager, A. Kaplan, Y. Kushnir, and M. A. Cane, 2009: Observed strengthening of the zonal sea surface temperature gradient across the equatorial Pacific Ocean. J. Clim., 22, 4316-4321.
Karpechko, A. Y., 2010: Uncertainties in future climate attributable to uncertainties in future Northern Annular Mode trend. Geophys. Res. Lett., 37, doi: 10.1029/2010gl044717.

Karpechko, A. Y., and E. Manzini, 2012: Stratospheric influence on tropospheric climate change in the Northern Hemisphere. J. Geophys. Res., 117, doi: 10.1029/2011JD017036.

Karpechko, A. Y., N. P. Gillett, L. J. Gray, and M. Dall'Amico, 2010: Influence of ozone recovery and greenhouse gas increases on Southern Hemisphere circulation. J. Geophys. Res., 115, D22117.

Kaspari, S., P. A. Mayewski, D. A. Dixon, V. B. Spikes, S. B. Sneed, M. J. Handley, and G. S. Hamilton, 2004: Climate variability in West Antarctica derived from annual accumulation-rate records from ITASE firn/ice cores. Annals of Glaciology, 39, 585-594.

Kattsov, V. M., J. E. Walsh, W. L. Chapman, V. A. Govorkova, T. V. Pavlova, and X. D. Zhang, 2007: Simulation and projection of arctic freshwater budget components by the IPCC AR4 global climate models. J. Hydrometeorol., 8, 571-589.

Kaufman, D. S., et al., 2009: Recent warming reverses long-term Arctic cooling. Science, 325, 1236-1239.

Kawatani, Y., K. Hamilton, and S. Watanabe, 2011: The Quasi-Biennial Oscillation in a double $\mathrm{CO}_{2}$ climate. J. Atmos. Sci., 68, 265-283.

Kawatani, Y., K. Hamilton, and A. Noda, 2012: The effects of changes in sea surface temperature and $\mathrm{CO}_{2}$ concentration on the Quasi-Biennial Oscillation. J. Atmos. Sci. 69, 1734-1749.

Kawazoe, S., and W. Gutowski, 2013: Regional, very heavy daily precipitation in NARCCAP simulations. J. Hydrometeorol., doi:10.1175/jhm-d-12-068.1.

Keenlyside, N., and M. Latif, 2007: Understanding equatorial Atlantic interannual variability. J. Clim., 20, 131-142.

Keenlyside, N., M. Latif, J. Jungclaus, L. Kornblueh, and E. Roeckner, 2008: Advancing decadal-scale climate prediction in the North Atlantic sector. Nature, 453 , 84-88.

Khain, A., B. Lynn, and J. Dudhia, 2010: Aerosol effects on intensity of landfalling hurricanes as seen from simulations with the WRF model with spectral bin microphysics. J. Atmos. Sci., 67, 365-384.

Khain, A., N. Cohen, B. Lynn, and A. Pokrovsky, 2008: Possible aerosol effects on lightning activity and structure of hurricanes. J. Atmos. Sci., 65, 3652-3677.

Kharin, V. V., F. W. Zwiers, X. Zhang, and G. C. Hegerl, 2007: Changes in temperature and precipitation extremes in the IPCC ensemble of global coupled model simulations. J. Clim., 20, 1419-1444.

Kidson, J. W., and J. A. Renwick, 2002: Patterns of convection in the tropical Pacific and their influence on New Zealand weather. Int. J. Climatol., 22, 151-174.

Kidston, J., and E. P. Gerber, 2010: Intermodel variability of the poleward shift of the austral jet stream in the CMIP3 integrations linked to biases in 20th century climatology. Geophys. Res. Lett., 37.

Kidston, J., J. A. Renwick, and J. McGregor, 2009: Hemispheric-scale seasonality of the Southern Annular Mode and impacts on the climate of New Zealand. J. Clim., 22, 4759-4770.

Kidston, J., G. K. Vallis, S. M. Dean, and J. A. Renwick, 2011: Can the increase in the eddy length scale under global warming cause the poleward shift of the jet streams? J. Clim., 24, 3764-3780.

Kim, B. M., and S. I. An, 2011: Understanding ENSO regime behavior upon an Increase in the warm-pool temperature using a simple ENSO model. J. Clim., $24,1438-1450$.

Kim, D., and H. Byun, 2009: Future pattern of Asian drought under global warming scenario. Theor. Appl. Climatol., 98, 137-150.

Kim, S. T., and J.-Y. Yu, 2012: The two types of ENSO in CMIP5 models. Geophys. Res. Lett., doi:10.1029/2012GL052006.

Kitoh, A., and T. Uchiyama, 2006: Changes in onset and withdrawal of the East Asian summer rainy season by multi-model global warming experiments. J. Meteorol. Soc. Jpn., 84, 247-258.

Kitoh, A., and S. Kusunoki, 2008: East Asian summer monsoon simulation by a 20-km mesh AGCM. Clim. Dyn., 31, 389-401.

Kitoh, A., S. Kusunoki, and T. Nakaegawa, 2011: Climate change projections over South America in the late 21st century with the 20 and $60 \mathrm{~km}$ mesh Meteorological Research Institute atmospheric general circulation model (MRIAGCM). J. Geophys. Res. Atmos., 116, D06105.

Kitoh, A., T. Ose, K. Kurihara, S. Kusunoki, M. Sugi, and KAKUSHIN Team-3 Modeling Group, 2009: Projection of changes in future weather extremes using superhigh-resolution global and regional atmospheric models in the KAKUSHIN Program: Results of preliminary experiments. Hydrol. Res. Lett., 3, 49-53. 
Kitoh, A., H. Endo, K. Krishna Kumar, I. F. A. Cavalcanti, P. Goswami, and T. Zhou, 2013: Monsoons in a changing world regional perspective in a global context. J. Geophys. Res. Atmos., 118, doi: 10.1002/jgrd.50258.

Kjellstrom, E., G. Nikulin, U. Hansson, G. Strandberg, and A. Ullerstig, 2011: 21st century changes in the European climate: Uncertainties derived from an ensemble of regional climate model simulations. Tellus $A, 63,24-40$.

Kjellström, E., P. Thejll, M. Rummukainen, J. H. Christensen, F. Boberg, C. O. B, and C. Fox Maule, 2013: Emerging regional climate change signals for Europe under varying large-scale circulation conditions. Clim. Res., 56, 103-119.

Klein, S. A., B. J. Soden, and N.-C. Lau, 1999: Remote sea surface temperature variations during ENSO: Evidence for a tropical atmospheric bridge. J. Clim., 12, 917-932.

Klingaman, N. P., S. J. Woolnough, H. Weller, and J. M. Slingo, 2011: The impact of finer-resolution air-sea coupling on the Intraseasonal Oscillation of the Indian monsoon. J. Clim., 24, 2451-2468.

Knight, J., 2009: The Atlantic Multidecadal Oscillation inferred from the forced climate response in coupled general ciculation models. J. Clim., 22, 1610-1625.

Knight, J. R., R. J. Allan, C. K. Folland, M. Vellinga, and M. E. Mann, 2005: A signature of persistent natural thermohaline circulation cycles in observed climate. Geophys. Res. Lett., 32, L20708.

Knutson, T. R., and R. E. Tuleya, 2004: Impact of $\mathrm{CO}_{2}$-induced warming on simulated hurricane intensity and precipitation: Sensitivity to the choice of climate model and convective parameterization. J. Clim., 17, 3477-3495.

Knutson, T. R., et al., 2006: Assessment of twentieth-century regional surface temperature trends using the GFDL CM2 coupled models. J. Clim., 19, 16241651.

Knutson, T. R., et al., 2010: Tropical cyclones and climate change. Nature Geosci., 3, 157-163.

Knutson, T. R., et al., 2013: Dynamical downscaling projections of 21st century Atlantic hurricane activity: CMIP3 and CMIP5 model-based scenarios. J. Clim., 26, 6591-6617.

Kodama, C., and T. Iwasaki, 2009: Influence of the SST rise on baroclinic instability wave activity under an aquaplanet condition. J. Atmos. Sci., 66, 2272-2287.

Kodera, K., M. E. Hori, S. Yukimoto, and M. Sigmond, 2008: Solar modulation of the Northern Hemisphere winter trends and its implications with increasing $\mathrm{CO2}$. Geophys. Res. Lett., 35, doi: 10.1029/2007gl031958.

Koenigk, T., R. Döscher, and G. Nikulin, 2011: Arctic future scenario experiments with a coupled regional climate model. Tellus $A, 63,69-86$.

Kohler, M., N. Kalthoff, and C. Kottmeier, 2010: The impact of soil moisture modifications on CBL characteristics in West Africa: A case-study from the AMMA campaign. Q. J. R. Meteorol. Soc., 136, 442-455.

Koldunov, N. V., D. Stammer, and J. Marotzke, 2010: Present-day Arctic sea ice variability in the Coupled ECHAM5/MPI-OM Model. J. Clim., 23, 2520-2543.

Kripalani, R., J. Oh, and H. Chaudhari, 2007a: Response of the East Asian summer monsoon to doubled atmospheric $\mathrm{CO2}$ : Coupled climate model simulations and projections under IPCC AR4. Theor. Appl. Climatol., 87, 1-28.

Kripalani, R. H., J. H. Oh, A. Kulkarni, S. S. Sabade, and H. S. Chaudhari, 2007b: South Asian summer monsoon precipitation variability: Coupled climate model simulations and projections under IPCC AR4. Theor. Appl. Climatol., 90, 133-159.

Krishnamurthy, C. K. B., U. Lall, and H. H. Kwon, 2009: Changing frequency and intensity of rainfall extremes over India from 1951 to 2003. J. Clim., 22, 47374746.

Krishnamurthy, V., and R. S. Ajayamohan, 2010: Composite structure of monsoon low pressure systems and its relation to Indian rainfall. J. Clim., 23, 4285-4305.

Kruger, L. F., R. P. da Rocha, M. S. Reboita, and T. Ambrizzi, 2011: RegCM3 nested in the HadAM3 scenarios A2 and B2: projected changes in cyclogeneses, temperature and precipitation over South Atlantic Ocean. Clim. Change, 113, 599-621.

Kucharski, F., A. Bracco, J. Yoo, A. Tompkins, L. Feudale, P. Ruti, and A. Dell'Aquila, 2009a: A Gill-Matsuno-type mechanism explains the tropical Atlantic influence on African and Indian monsoon rainfall. Q. J. R. Meteorol. Soc., 135, 569-579.

Kucharski, F., et al., 2009b: The CLIVAR C20C project: Skill of simulating Indian monsoon rainfall on interannual to decadal timescales. Does GHG forcing play a role? Clim. Dyn., 33, 615-627.

Kug, J.-S., and I.-S. Kang, 2006: Interactive Feedback between ENSO and the Indian Ocean. J. Clim., 19, 1784-1801.

Kug, J.-S., F.-F. Jin, and S.-I. An, 2009: Two types of El Nino events: Cold tongue El Niño and warm pool El Niño. J. Clim., 22, 1499-1515.
Kug, J. S., S. I. An, Y. G. Ham, and I. S. Kang, 2010: Changes in El Niño and La Niña teleconnections over North Pacific-America in the global warming simulations. Theor. Appl. Climatol., 100, 275-282.

Kulkarni, A., 2012: Weakening of Indian summer monsoon rainfall in warming environment. Theor. Appl. Climatol., doi:10.1007/s00704-012-0591-4.

Kumar, K., S. Patwardhan, A. Kulkarni, K. Kamala, K. Rao, and R. Jones, 2011 a: Simulated projections for summer monsoon climate over India by a highresolution regional climate model (PRECIS). Curr. Sci., 101, 312-326.

Kumar, K., et al., $2011 \mathrm{~b}$ : The once and future pulse of Indian monsoonal climate. Clim. Dyn., 36, 2159-2170.

Kumar, K. K., B. Rajagopalan, M. Hoerling, G. Bates, and M. Cane, 2006a: Unraveling the mystery of Indian Monsoon failure during El Niño. Science, 314, 115-119.

Kumar, P., et al., 2013: Downscaled climate change projections with uncertainty assessment over India using a high resolution multi-model approach. Sci. Total Environ., doi:10.1016/j.scitotenv.2013.01.051.

Kumar, V., R. Deo, and V. Ramachandran, 2006b: Total rain accumulation and rainrate analysis for small tropical Pacific islands: A case study of Suva, Fiji. Atmos. Sci. Lett., 7, 53-58.

Kusunoki, S., and R. Mizuta, 2008: Future changes in the Baiu rain band projected by a 20-km mesh global atmospheric model: Sea surface temperature dependence. Sola, 4, 85-88.

Kusunoki, S., and 0. Arakawa, 2012: Change in the precipitation intensity of the East Asian summer monsoon projected by CMIP3 models. Clim. Dyn., 38, 2055-2072.

Kuzmina, S. I., L. Bengtsson, O. M. Johannessen, H. Drange, L. P. Bobylev, and M. W. Miles, 2005: The North Atlantic Oscillation and greenhouse-gas forcing. Geophys. Res. Lett., 32, doi: 10.1029/2004gl021064.

Kvamsto, N., P. Skeie, and D. Stephenson, 2004: Impact of labrador sea-ice extent on the North Atlantic oscillation. Int. J. Climatol., 24, 603-612.

Kwok, R., and J. C. Comiso, 2002: Southern ocean climate and sea ice anomalies associated with the Southern Oscillation. J. Clim., 15, 487-501.

L'Heureux, M. L., and D. W. J. Thompson, 2006: Observed relationships between the El Niño-Southern Oscillation and the extratropical zonal-mean circulation. J. Clim., 19, 276-287.

L'Heureux, M. L., and R. W. Higgins, 2008: Boreal winter links between the MaddenJulian oscillation and the Arctic oscillation. J. Clim., 21, 3040-3050.

Laine, A., M. Kageyama, D. Salas-Melia, G. Ramstein, S. Planton, S. Denvil, and S. Tyteca, 2009: An energetics study of wintertime Northern Hemisphere storm tracks under $4 \times \mathrm{CO}(2)$ conditions in two ocean-atmosphere coupled models. J. Clim., 22, 819-839.

Lam, H., M. H. Kok, and K. K. Y. Shum, 2012: Benefits from typhoons-the Hong Kong perspective. Weather, 67, 16-21.

Lambert, S. J., and J. C. Fyfe, 2006: Changes in winter cyclone frequencies and strengths simulated in enhanced greenhouse warming experiments: Results from the models participating in the IPCC diagnostic exercise. Clim. Dyn., 26, $713-728$.

Landsea, C. W., R. A. Pielke, A. Mestas-Nunez, and J. A. Knaff, 1999: Atlantic basin hurricanes: Indices of climatic changes. Clim. Change, 42, 89-129.

Lang, C., and D. W. Waugh, 2011: Impact of climate change on the frequency of Northern Hemisphere summer cyclones. J. Geophys. Res. Atmos., 116, D04103.

Langenbrunner, B., and J. D. Neelin, 2013: Analyzing ENSO teleconnections in CMIP models as a measure of model fidelity in simulating precipitation. J. Clim., doi:10.1175/jcli-d-12-00542.1.

Lapp, S. L., J. M. St. Jacques, E. M. Barrow, and D. J. Sauchyn, 2012: GCM projections for the Pacifi Decadal Oscillation under greenhouse forcing for the early $21 \mathrm{st}$ century. International Journal of Climatology, 32, 1423-1442.

Lau, K., S. Shen, K. Kim, and H. Wang, 2006: A multimodel study of the twentiethcentury simulations of Sahel drought from the 1970s to 1990s. J. Geophys. Res. Atmos., 111.

Lau, K., et al., 2008: The Joint Aerosol-Monsoon Experiment—A new challenge for monsoon climate research. Bull. Am. Meteorol. Soc., doi:10.1175/BAMS-89-3369, 369-383.

Lau, K. M., and H. T. Wu, 2007: Detecting trends in tropical rainfall characteristics, 1979-2003. Int. J. Climatol., 27, 979-988.

Lau, N.-C., and M. J. Nath, 2012: A model study of heat waves over North America: Meteorological aspects and projections for the 21st Century. J. Clim., 25, 47614784.

Lavender, S., and K. Walsh, 2011: Dynamically downscaled simulations of Australian region tropical cyclones in current and future climates. Geophys. Res. Lett., 38, doi: 10.1029/2011GL047499. 
Leckebusch, G. C., U. Ulbrich, L. Froehlich, and J. G. Pinto, 2007: Property loss potentials for European midlatitude storms in a changing climate. Geophys. Res. Lett., 34, doi: 10.1029/2006gl027663.

Leckebusch, G. C., B. Koffi, U. Ulbrich, J. G. Pinto, T. Spangehl, and S. Zacharias, 2006: Analysis of frequency and intensity of European winter storm events from a multi-model perspective, at synoptic and regional scales. Clim. Res., 31, 59-74.

Lee, J. N., S. Hameed, and D. T. Shindell, 2008: The northern annular mode in summer and its relation to solar activity variations in the GISS ModelE. J. Atmos. Sol. Terres. Phys., 70, 730-741.

Lee, T.-C., K.-Y. Chan, H.-S. Chan, and M.-H. Kok, 2011: Projections of extreme rainfall in Hong Kong in the 21st century. Acta Meteorol. Sin., 25, 691-709.

Lee, T.-C., T. R. Knutson, H. Kamahori, and M. Ying, 2012: Impacts of climate change on tropical cyclones in the western North Pacific basin. Part I: Past observations. Trop. Cyclone Res. Rev., 1, 213-230.

Lelieveld, J., et al., 2012: Climate change and impacts in the Eastern Mediterranean and the Middle East. Clim. Change, 114, 667-687.

Leslie, L., D. Karoly, M. Leplastrier, and B. Buckley, 2007: Variability of tropical cyclones over the southwest Pacific Ocean using a high-resolution climate model. Meteorol. Atmos. Phys., 97, 171-180.

Leung, L. R., and Y. Qian, 2009: Atmospheric rivers induced heavy precipitation and flooding in the western U.S. simulated by the WRF regional climate model. Geophys. Res. Lett., 36, L03820.

Levine, R. C., and A. G. Turner, 2012: Dependence of Indian monsoon rainfall on moisture fluxes across the Arabian Sea and the impact of coupled model sea surface temperature biases. Clim. Dyn., 38, 2167-2190.

Levitus, S., G. Matishov, D. Seidov, and I. Smolyar, 2009: Barents Sea multidecadal variability. Geophys. Res. Lett., 36, L19604.

Li, B., and T. J. Zhou, 2011: El Nino-Southern Oscillation-related principal interannual variability modes of early and late summer rainfall over East Asia in sea surface temperature-driven atmospheric general circulation model simulations. J. Geophys. Res. Atmos., 116, 15.

Li, G., and B. Ren, 2012: Evidence for strengthening of the tropical Pacific ocean surface wind speed during 1979-2001. Theor. Appl. Climatol., doi:10.1007/ s00704-0110-463-3.

Li, H., A. Dai, T. Zhou, and J. Lu, 2010a: Responses of East Asian summer monsoon to historical SST and atmospheric forcing during 1950-2000. Clim. Dyn., 34, 501-514.

Li, H. M., L. Feng, and T. J. Zhou, 2011a: Multi-model projection of July-August climate extreme changes over China under $\mathrm{CO}_{2}$ doubling. Part II: Temperature. Adv. Atmos. Sci., 28, 448-463.

Li, H. M., L. Feng, and T. J. Zhou, 2011b: Multi-model projection of July-August climate extreme changes over China under $\mathrm{CO}_{2}$ doubling. Part I: precipitation. Adv. Atmos. Sci., 28, 433-447.

Li, J., and J. Wang, 2003: A modified zonal index and its physical sense. Geophys. Res. Lett., 30, doi: 10.1029/2003GL017441.

Li, J., J. Feng, and Y. Li, 2012a: A possible cause of decreasing summer rainfall in northeast Australia. Int. J. Climatol., 32, 995-1005.

Li, J. B., et al., $2011 \mathrm{c}$ : Interdecadal modulation of El Nino amplitude during the past millennium. Nature Clim. Change, 1, 114-118.

Li, T., P. Liu, X. Fu, B. Wang, and G. Meehl, 2006: Spatiotemporal structures and mechanisms of the tropospheric biennial oscillation in the Indo-Pacific warm ocean regions. J. Clim., 19, 3070-3087.

Li, T., M. Kwon, M. Zhao, J. Kug, J. Luo, and W. Yu, 2010b: Global warming shifts Pacific tropical cyclone location. Geophys. Res. Lett., 37, doi: 10.1029/2010GL045124.

Li, Y., and N. Lau, 2012: Impact of ENSO on the atmospheric variability over the north Atlantic in late winter-Role of transient eddies. J. Clim., 25, 320-342.

Li, Y., N. C. Jourdain, A. S. Taschetto, C. C. Ummenhofer, K. Ashok, and A. Sen Gupta, 2012b: Evaluation of monsoon seasonality and the tropospheric biennial oscillation transitions in the CMIP models. Geophys. Res. Lett., 39, L20713.

Liang, X.-Z., K. E. Kunkel, G. A. Meehl, R. G. Jones, and J. X. L. Wang, 2008a: Regional climate models downscaling analysis of general circulation models present climate biases propagation into future change projections. Geophys. Res. Lett., 35, L08709.

Liang, X.-Z., J. Zhu, K. E. Kunkel, M. Ting, and J. X. L. Wang, 2008b: Do GCMs simulate the North American monsoon precipitation seasonal-interannual variability? J. Clim., 21, 4424-4448.

Lienert, F., J. C. Fyfe, and W. J. Marryfield, 2011: Do climate models capture the tropical influences on North Pacific sea surface temperature variability? J. Clim., 24, 6203-6209.
Lim, E.-P., and I. Simmonds, 2009: Effect of tropospheric temperature change on the zonal mean circulation and SH winter extratropical cyclones. Clim. Dyn., 33, $19-32$.

Lim, Y.-K., L. B. Stefanova, S. C. Chan, S. D. Schubert, and J. J. O'Brien, 2011: High-resolution subtropical summer precipitation derived from dynamical downscaling of the NCEP/DOE reanalysis:how much small-scale information is added by a regional model? Clim. Dyn., 37, 1061-1080.

Lima, K., P. Satyamurty, and J. Fernández, 2010: Large-scale atmospheric conditions associated with heavy rainfall episodes in Southeast Brazil. Theor. Appl. Climatol., $101,121-135$.

Lin, H., G. Brunet, and J. Derome, 2009: An observed connection between the North Atlantic Oscillation and the Madden-Julian Oscillation. J. Clim., 22, 364-380.

Lin, J. L., et al., 2008a: Subseasonal variability associated with Asian summer monsoon simulated by 14 IPCC AR4 coupled GCMs. J. Clim., 21, 4541-4567.

Lin, J. L., et al., 2008b: North American monsoon and convectively coupled equatorial waves simulated by IPCC AR4 coupled GCMs. J. Clim., 21, 2919-2937.

Linkin, M., and S. Nigam, 2008: The North Pacific Oscillation-West Pacific teleconnection pattern: Mature-phase structure and winter impacts. J. Clim., 21, 1979-1997.

Lintner, B., and J. Neelin, 2008: Eastern margin variability of the South Pacific Convergence Zone. Geophys. Res. Lett., 35, doi: 10.1029/2008gl034298.

Lionello, P., S. Planton, and X. Rodo, 2008: Preface: Trends and climate change in the Mediterranean region. Global Planet. Change, 63, 87-89.

Liu, H. W., T. J. Zhou, Y. X. Zhu, and Y. H. Lin, 2012a: The strengthening East Asia summer monsoon since the early 1990s. Chinese Science Bulletin, 57, 15531558.

Liu, J., J. A. Curry, H. Wang, M. Song, and R. M. Horton, 2012b: Impact of declining Arctic sea ice on winter snowfall. Proc. Natl. Acad. Sci. U.S.A., 109, 4074-4079.

Liu, J. P., and J. A. Curry, 2006: Variability of the tropical and subtropical ocean surface latent heat flux during 1989-2000. Geophys. Res. Lett., 33, doi: $10.1029 / 2005 \mathrm{gl} 024809$.

Liu, Y., S.-K. Lee, B. A. Muhling, J. T. Lamkin, and D. B. Enfield, 2012c: Significant reduction of the Loop Current in the 21st century and its impact on the Gulf of Mexico. J. Geophys. Res., 117, C05039.

Liu, Z., and B. Huang, 2000: Cause of tropical Pacific warming trend. Geophys. Res. Lett., 27, 1935-1938.

Liu, Z., S. Vavrus, F. He, N. Wen, and Y. Zhong, 2005: Rethinking tropical ocean response to global warming: The enhanced equatorial warming. J. Clim., 18, 4684-4700.

Lockwood, M., R. G. Harrison, T. Woollings, and S. K. Solanki, 2010: Are cold winters in Europe associated with low solar activity? Environ. Res. Lett., 5, doi: 10.1088/1748-9326/5/2/024001.

Loeptien, U., O. Zolina, S. Gulev, M. Latif, and V. Soloviov, 2008: Cyclone life cycle characteristics over the Northern Hemisphere in coupled GCMs. Clim. Dyn., 31, 507-532.

Long, Z., W. Perrie, J. Gyakum, R. Laprise, and D. Caya, 2009: Scenario changes in the climatology of winter midlatitude cyclone activity over eastern North America and the Northwest Atlantic. J. Geophys. Res. Atmos., 114, doi: 10.1029/2008jd010869.

Lorenz, D. J., and D. L. Hartmann, 2003: Eddy-zonal flow feedback in the Northern Hemisphere winter. J. Clim., 16, 1212-1227.

Lorenz, D. J., and E. T. DeWeaver, 2007: Tropopause height and zonal wind response to global warming in the IPCC scenario integrations. J. Geophys. Res. Atmos., 112, doi: 10.1029/2006jd008087.

Lu, J., G. A. Vecchi, and T. Reichler, 2007: Expansion of the Hadley cell under global warming. Geophys. Res. Lett., 34, doi: 10.1029/2006gl028443.

Lu, J., G. Chen, and D. M. W. Frierson, 2008: Response of the zonal mean atmospheric circulation to El Nino versus global warming. J. Clim., 21, 5835-5851.

Lu, J., G. Chen, and D. M. W. Frierson, 2010: The position of the mid latitude storm track and eddy-driven westerlies in Aquaplanet AGCMs. J. Atmos. Sci., 67, 39844000.

Lu, R., and Y. Fu, 2010: Intensification of East Asian summer rainfall interannual variability in the twenty-first century simulated by 12 CMIP3 coupled models. J. Clim., doi:10.1175/2009JCLI3130.1, 3316-3331.

Lucarini, V., and F. Ragone, 2011: Energetics of climate models: Net energy balance and meridional enthalpy transport. Rev. Geophys. , 49, doi: 10.1029/2009RG000323.

Lucas, C., H. Nguyen, and B. Timbal, 2012: An observational analysis of southern hemisphere tropical expansion. J. Geophys. Res., 117, doi: 10.1029/2011JD017033. 
Luo, D., W. Zhou, and K. Wei, 2010: Dynamics of eddy-driven North Atlantic Oscillations in a localized shifting jet: Zonal structure and downstream blocking. Clim. Dyn., 34, 73-100.

Luo, Y., and L. M. Rothstein, 2011: Response of the Pacific ocean circulation to climate change. Atmosphere-ocean, 49, 235-244.

Lupo, A. R., I. I. Mokhov, M. G. Akperov, A. V. Chernokulsky, and H. Athar, 2012: A dynamic analysis of the role of the planetary- and synoptic-scale in the summer of 2010 blocking episodes over the European part of Russia. Adv. Meteorol., $2012,11$.

Ma, J., and S.-P. Xie, 2013: Regional patterns of sea surface temperature change: A source of uncertainty in future projections of precipitation and atmospheric circulation. J. Clim., 26, 2482-2501.

Magnusdottir, G., C. Deser, and R. Saravanan, 2004: The effects of North Atlantic SST and sea ice anomalies on the winter circulation in CCM3. Part I: Main features and storm track characteristics of the response. J. Clim., 17, 857-876.

Mahajan, S., R. Zhang, and T. L. Delworth, 2011: Impact of the Atlantic meridional overturning circulation (AMOC) on Arctic surface air temperature and sea ice variability. J. Clim., 24, 6573-6581.

Mahowald, N., 2007: Anthropocene changes in desert area: Sensitivity to climate model predictions. Geophys. Res. Lett., 34, doi: 10.1029/2007GL030472.

Malhi, Y., J. T. Roberts, R. A. Betts, T. J. Killeen, W. Li, and C. A. Nobre, 2008: Climate change, deforestation, and the fate of the Amazon. Science, 319, 169-172.

Maloney, E. D., and J. Shaman, 2008: Intraseasonal variability of the West African monsoon and Atlantic ITCZ. J. Clim., 21, 2898-2918.

Maloney, E. D., and S.-P. Xie, 2013: Sensitivity of MJO activity to the pattern of climate warming. J. Adv. Model. Earth Syst., 5, 32-47.

Manatsa, D., W. Chingombe, H. Matsikwa, and C. H. Matarira, 2008: The superior influence of Darwin Sea level pressure anomalies over ENSO as a simple drought predictor for Southern Africa. Theor. Appl. Climatol., 92, 1-14.

Mandke, S. K., A. K. Sahai, M. A. Shinde, S. Joseph, and R. Chattopadhyay, 2007: Simulated changes in active/break spells during the Indian summer monsoon due to enhanced $\mathrm{CO}_{2}$ concentrations: Assessment from selected coupled atmosphere-ocean global climate models. Int. J. Climatol, 27, 837-859.

Mann, M. E., and K. A. Emanuel, 2006: Atlantic hurricane trends linked to climate change. Eos Trans., 87, 233-241.

Manton, M. J., et al., 2001: Trends in extreme daily rainfall and temperature in Southeast Asia and the South Pacific: 1961-1998. Int. J. Climatol., 21, 269-284.

Mantua, N. J., S. R. Hare, Y. Zhang, J. M. Wallace, and R. C. Francis, 1997: A Pacific interdecadal climate oscillation with impacts on salmon production. Bull. Am. Meteorol. Soc., 78, 1069-1079.

Marcella, M. P., and E. A. B. Eltahir, 2011: Modeling the summertime climate of Southwest Asia: The role of land surface processes in shaping the climate of semiarid regions. J. Clim., 25, 704-719.

Marchant, R., C. Mumbi, S. Behera, and T. Yamagata, 2007: The Indian Ocean dipole-the unsung driver of climatic variability in East Africa. Afr. J. Ecol., 45, 4-16.

Marengo, J., et al., 2010a: Recent developments on the South American Monsoon system. Int. J. Climatol., 32, 1-21.

Marengo, J., et al., 2012: Development of regional future climate change scenarios in South America using the Eta CPTEC/HadCM3 climate change projections: Climatology and regional analyses for the Amazon, São Francisco and the Paraná River basins. Clim. Dyn., 38, 1829-1848.

Marengo, J. A., and C. C. Camargo, 2008: Surface air temperature trends in Southern Brazil for 1960-2002. Int. J. Climatol., 28, 893-904.

Marengo, J. A., R. Jones, L. M. Alves, and M. C. Valverde, 2009: Future change of temperature and precipitation extremes in South America as derived from the PRECIS regional climate modeling system. Int. J. Climatol., 29, 2241-2255.

Marengo, J. A., M. Rusticucci, O. Penalba, and M. Renom, 2010b: An intercomparison of observed and simulated extreme rainfall and temperature events during the last half of the twentieth century: Part 2: Historical trends. Clim. Change, 98, 509-529.

Mariotti, A., and A. Dell'Aquila, 2012: Decadal climate variability in the Mediterranean region: Roles of large-scale forcings and regional processes. Clim. Dyn., 38, $1129-1145$.

Marshall, A. G., and A. A. Scaife, 2009: Impact of the QBO on surface winter climate. J. Geophys. Res., 114, doi: 10.1029/ 2009jd011737.
Marshall, A. G., and A. A. Scaife, 2010: Improved predictability of stratospheric sudden warming events in an atmospheric general circulation model with enhanced stratospheric resolution. J. Geophys. Res. Atmos., 115, doi: 10.1029/2009jd012643.

Marshall, G. J., 2007: Half-century seasonal relationships between the Southern Annular Mode and Antarctic temperatures. Int. J. Climatol., 27, 373-383.

Martius, O., L. M. Polvani, and H. C. Davies, 2009: Blocking precursors to stratospheric sudden warming events. Geophys. Res. Lett., 36, L14806.

Marullo, S., V. Artale, and R. Santoleri, 2011: The SST multidecadal variability in the Atlantic-Mediterranean region and its relation to AMO. J. Clim., 24, 4385-4401.

Masato, G., B. J. Hoskins, and T. J. Woollings, 2012: Wave-breaking characteristics of midlatitude blocking. Q. J. R. Meteorol. Soc., 138, 1285-1296.

Masato, G., B. J. Hoskins, and T. Woollings, 2013: Winter and summer Northern Hemisphere blocking in CMIP5 models. J. Clim., doi:10.1175/jcli-d-12-00466.1.

Mason, S., 2001: El Nino, climate change, and Southern African climate. Environmetrics, 12, 327-345.

Massom, R. A., M. J. Pook, J. C. Comiso, N. Adams, J. Turner, T. Lachlan-Cope, and T. T. Gibson, 2004: Precipitation over the interior East Antarctic ice sheet related to midlatitude blocking-high activity. J. Clim., 17, 1914-1928.

Matsueda, M., 2011: Predictability of Euro-Russian blocking in summer of 2010. Geophys. Res. Lett., 38, L06801.

Matsueda, M., H. Endo, and R. Mizuta, 2010: Future change in Southern Hemisphere summertime and wintertime atmospheric blockings simulated using a 20-km-mesh AGCM. Geophys. Res. Lett., 37, L02803.

May, W., 2011: The sensitivity of the Indian summer monsoon to a global warming of 2 degrees C with respect to pre-industrial times. Clim. Dyn., 37, 1843-1868.

McCabe, G., and D. Wolock, 2010: Long-term variability in Northern Hemisphere snow cover and associations with warmer winters. Clim. Change, doi:10.1007/ s10584-009-9675-2, 141-153.

McDonald, R. E., 2011: Understanding the impact of climate change on Northern Hemisphere extra-tropical cyclones. Clim. Dyn., 37, 1399-1425.

McLandress, C., and T. G. Shepherd, 2009: Simulated anthropogenic changes in the Brewer-Dobson circulation, including its extension to high latitudes. J. Clim., 22, 1516-1540.

Mearns, L. O., R. Arritt, S. Biner, M. Bukovsky, S. Stain, and et al., 2012: The North American regional climate change assessment program: Overview of phase I results. Bull. Am. Meteorol. Soc., 93, 1337-1362.

Meehl, G., and H. Teng, 2007: Multi-model changes in El Nino teleconnections over North America in a future warmer climate. Clim. Dyn., 29, 779-790.

Meehl, G., J. Arblaster, and W. Collins, 2008: Effects of Black Carbon Aerosols on the Indian Monsoon. J. Clim., 21, 2869-2882.

Meehl, G., A. Hu, and C. Tebaldi, 2010: Decadal Prediction in the Pacific Region. J. Clim., 23, 2959-2973.

Meehl, G. A., 1997: The south Asian monsoon and the tropospheric biennial oscillation. J. Clim., 10, 1921-1943.

Meehl, G. A., and A. Hu, 2006: Megadroughts in the Indian monsoon region and southwest North America and a mechanism for associated multidecadal Pacific sea surface temperature anomalies. J. Clim., 19, 1605-1623.

Meehl, G. A., and J. M. Arblaster, 2012: Relating the strength of the tropospheric biennial oscillation (TBO) to the phase of the Interdecadal Pacific Oscillation (IPO). Geophys. Res. Lett., 39, L20716.

Meehl, G. A., et al., 2007: Global climate projections. In: Climate Change 2007: The Physical Science Basis. Contribution of Working Group I to the Fourth Assessment Report of the Intergovernmental Panel on Climate Change [Solomon, S., D. Qin, M. Manning, Z. Chen, M. Marquis, K. B. Averyt, M. Tignor and H. L. Miller (eds.)] Cambridge University Press, Cambridge, United Kingdom and New York, NY, USA, pp. 747-846.

Menary, M., W. Park, K. Lohmann, M. Vellinga, M. Palmer, M. Latif, and J. Jungclaus, 2012: A multimodel comparison of centennial Atlantic meridional overturning circulation variability. Clim. Dyn., 38, 2377-2388.

Mendes, M. C. D., R. M. Trigo, I. F. A. Cavalcanti, and C. C. Da Camara, 2008: Blocking episodes in the Southern Hemisphere: Impact on the climate of adjacent continental areas. Pure Appl. Geophys., 165, 1941-1962.

Mendez, M., and V. Magana, 2010: Regional aspects of prolonged meteorological droughts over Mexico and Central America. J. Clim., 23, 1175-1188.

Mendoza, B., V. Garcia-Acosta, V. Velasco, E. Jauregui, and R. Diaz-Sandoval, 2007: Frequency and duration of historical droughts from the 16th to the 19th centuries in the Mexican Maya lands, Yucatan Peninsula. Clim. Change, 83, 151-168. 
Meneghini, B., I. Simmonds, and I. N. Smith, 2007: Association between Australian rainfall and the Southern Annular Mode. Int. J. Climatol., 27, 109-121.

Menendez, C. G., and A. Carril, 2010: Potential changes in extremes and links with the Southern Annular Mode as simulated by a multi-model ensemble. Clim. Change, 98, 359-377.

Metcalfe, S. E., M. D. Jones, S. J. Davies, A. Noren, and A. MacKenzie, 2010: Climate variability over the last two millennia in the North American Monsoon, recorded in laminated lake sediments from Laguna de Juanacatlan, Mexico. Holocene, 20, 1195-1206.

Miller, G. H., et al., 2010: Temperature and precipitation history of the Arctic. Q. Sci. Rev., 29, 1679-1715.

Miller, R. L., G. A. Schmidt, and D. T. Shindell, 2006: Forced annular variations in the 20th century intergovernmental panel on climate change fourth assessment report models. J. Geophys. Res. Atmos., 111, doi: 10.1029/2005jd006323.

Minvielle, M., and R. D. Garreaud, 2011: Projecting rainfall changes over the South American altiplano. J. Clim., 24, 4577-4583.

Mitas, C. M., and A. Clement, 2005: Has the Hadley cell been strengthening in recent decades? Geophys. Res. Lett., 32, doi: 10.1029/2004gl021765.

Mitas, C. M., and A. Clement, 2006: Recent behavior of the Hadley cell and tropical thermodynamics in climate models and reanalyses. Geophys. Res. Lett., 33, doi: $10.1029 / 2005 \mathrm{gl} 024406$.

Mitchell, T. D., and P. D. Jones, 2005: An improved method of constructing a database of monthly climate observations and associated high-resolution grids. Int. J. Climatol., 25, 693-712.

Mitchell, T. P., and J. M. Wallace, 1996: ENSO seasonality: 1950-78 versus 1979-92. J. Clim., 9, 3149-3161.

Mizuta, R., 2012: Intensification of extratropical cyclones associated with the polar jet change in the CMIP5 global warming projections. Geophys. Res. Lett., 39, doi: 10.1029/2012GL053032.

Mizuta, R., M. Matsueda, H. Endo, and S. Yukimoto, 2011: Future change in extratropical cyclones associated with change in the upper troposphere. J. Clim., 24, 6456-6470.

Mizuta, R., et al., 2012: Climate simulations using MRI-AGCM3.2 with 20-km grid. J. Meteorol. Soc. Jpn., 90A, 233-258.

Mock, C. J., and A. R. Brunelle-Daines, 1999: A modern analogue of western United States summer palaeoclimate at 6000 years before present. Holocene, 9, 541545.

Mohino, E., S. Janicot, and J. Bader, 2011: Sahel rainfall and decadal to multi-decadal sea surface temperature variability. Clim. Dyn., 37, 419-440.

Moise, A., and F. Delage, 2011: New climate model metrics based on objectorientated pattern matching of rainfall. J. Geophys. Res. Atmos., 116, doi: 10.1029/2010JD015318.

Moise, A. F., R. A. Colman, and J. R. Brown, 2012: Behind uncertainties in projections of Australian tropical climate: Analysis of 19 CMIP3 models. J. Geophys. Res. Atmos., 117, doi: 10.1029/2011jd017365.

Moise, A. F., R. A. Colman, and H. Zhang, 2005: Coupled model simulations of current Australian surface climate and its changes under greenhouse warming: An analysis of 18 CMIP2 models. Aust. Meteorol. Mag., 54, 291-307.

Monaghan, A. J., and D. H. Bromwich, 2008: Advances in describing recent Antarctic climate variablity. Bull. Am. Meteorol. Soc., 89, 1295-1306.

Monahan, A. H., L. Pandolfo, and J. C. Fyfe, 2001: The preferred structure of variability of the Northern Hemisphere atmospheric circulation. Geophys. Res. Lett., 28, 1019-1022.

Monahan, A. H., J. C. Fyfe, M. H. P. Ambaum, D. B. Stephenson, and G. R. North, 2009: Empirical Orthogonal Functions: The medium is the message. J. Clim., 22, 6501-6514.

Moron, V., A. W. Robertson, and J.-H. Qian, 2010: Local versus regional-scale characteristics of monsoon onset and post-onset rainfall over Indonesia. Clim. Dyn., 34, 281-299.

Moss, R. H., et al., 2010: The next generation of scenarios for climate change research and assessment. Nature, 463, 747-756.

Muller, W. A., and E. Roeckner, 2006: ENSO impact on midlatitude circulation patterns in future climate change projections. Geophys. Res. Lett., 33, doi: 10.1029/2005gl025032.

Müller, W. A., and E. Roeckner, 2008: ENSO teleconnections in projections of future climate in ECHAM5/MPI-OM. Clim. Dyn., 31, 533-549.

Murakami, H., and B. Wang, 2010: Future change of North Atlantic tropical cyclone tracks: Projection by a 20-km-mesh global atmospheric model. J. Clim., 23, 2699-2721.
Murakami, H., and M. Sugi, 2010: Effect of model resolution on tropical cyclone climate projections. Sola, 6, 73-76.

Murakami, H., B. Wang, and A. Kitoh, 2011 a: Future change of Western North Pacific typhoons: Projections by a 20-km-mesh global atmospheric model. J. Clim., 24, 1154-1169.

Murakami, H., R. Mizuta, and E. Shindo, 2011 b: Future changes in tropical cyclone activity projected by multi-physics and multi-SST ensemble experiments using the 60-km-mesh MRI-AGCM. Clim. Dyn., doi:10.1007/s00382-011-1223-x.

Murakami, H., M. Sugi, and A. Kitoh, 2013: Future changes in tropical cyclone activity in the North Indian Ocean projected by high-resolution MRI-AGCMs. Clim. Dyn., 40, 1949-1968.

Murakami, H., et al., 2012: Future changes in tropical cyclone activity projected by the new high-resolution MRI-AGCM. J. Clim., 25, 3237-3260.

Murphy, B. F., and B. Timbal, 2008: A review of recent climate variability and climate change in southeastern Australia. Int. J. Climatol., 28, 859-879.

Muza, M. N., L. M. V. Carvalho, C. Jones, and B. Liebmann, 2009: Intraseasonal and interannual variability of extreme dry and wet events over southeastern South America and the subtropical Atlantic during austral summer. J. Clim., 22, 16821699.

Nanjundiah, R., V.Vidyunmala, and J. Srinivasan, 2005: The impact of increase in $\mathrm{CO}_{2}$ on the simulation of tropical biennial oscillations (TBO) in 12 coupled general circulation models. Atmos. Sci. Lett., 6, 183-191.

Neelin, J., C. Chou, and H. Su, 2003: Tropical drought regions in global warming and El Nino teleconnections. Geophys. Res. Lett., 30, doi: 10.1029/2003GL018625.

Neelin, J. D., M. Munnich, H. Su, J. E. Meyerson, and C. E. Holloway, 2006: Tropical drying trends in global warming models and observations. Proc. Natl. Acad. Sci., 103, 6110-6115.

Neelin, J. D., B. Langenbrunner, J. E. Meyerson, A. Hall, and N. Berg, 2013: California winter precipitation change under global warming in CMIP5 models. J. Clim., 26, 6238-6256.

Nguyen, K., J. Katzfey, and J. McGregor, 2012: Global $60 \mathrm{~km}$ simulations with CCAM: Evaluation over the tropics. Clim. Dyn., 39, 637-654.

Nicholls, N., C. Landsea, and J. Gill, 1998: Recent trends in Australian region tropical cyclone activity. Meteorol. Atmos. Phys., 65, 197-205.

Nieto-Ferreira, R., and T. Rickenbach, 2010: Regionality of monsoon onset in South America: A three-stage conceptual model. Int. J. Climatol., 31, 1309-1321.

Nigam, S., 2003: Teleconnections. In: Encyclopedia of Atmospheric Sciences [J. A. P. J. R. Holton and J. A. Curry (eds.)]. Academic Press, San Diego, CA, USA, pp. 2243-2269.

Ninomiya, K., 2012: Characteristics of intense rainfalls over southwestern Japan in the Baiu season in the CMIP3 20th century simulation and 21st century projection. J. Meteorol. Soc. Jpn., 90A, 327-338.

Niyogi, D., C. Kishtawal, S. Tripathi, and R. S. Govindaraju, 2010: Observational evidence that agricultural intensification and land use change may be reducing the Indian summer monsoon rainfall. Water Resources Research, 46, W03533, doi: 03510.01029/02008wr007082.

Nuñez, M. N., S. A. Solman, and M. F. Cabre, 2009: Regional climate change experiments over southern South America. II: Climate change scenarios in the late twenty-first century. Clim. Dyn., 32, 1081-1095.

O'Gorman, P. A., 2010: Understanding the varied response of the extratropical storm tracks to climate change. Proc. Natl. Acad. Sci. U.S.A., 107, 19176-19180.

O'Gorman, P. A., and T. Schneider, 2008: Energy of midlatitude transient eddies in idealized simulations of changed climates. J. Clim., 21, 5797-5806.

Okamoto, K., K. Sato, and H. Akiyoshi, 2011: A study on the formation and trend of the Brewer-Dobson circulation. J. Geophys. Res., 116, doi: 10.1029/2010JD014953.

Okumura, Y. M., D. Schneider, C. Deser, and R. Wilson, 2012: Decadal-interdecadal climate variability over Antarctica and linkages to the tropics: Analysis of ice core, instrumental, and tropical proxy data. J. Clim., 25, 7421-7441.

Onol, B., and F. Semazzi, 2009: Regionalization of climate change simulations over the Eastern Mediterranean. J. Clim., 22, 1944-1961.

Orlowsky, B., and S. Seneviratne, 2012: Global changes in extreme events: Regional and seasonal dimension. Clim. Change, 110, 669-696.

Ose, T., and O. Arakawa, 2011: Uncertainty of future precipitation change due to global warming associated with sea surface temperature change in the tropical Pacific. J. Meteorol. Soc. Jpn., 89, 539-552.

Oshima, K., Y. Tanimoto, and S. P. Xie, 2012: Regional patterns of wintertime SLP change over the North Pacific and their uncertainty in CMIP3 multi-model projections. J. Meteorol. Soc. Jpn., 90, 385-396. 
Ouzeau, G., J. Cattiaux, H. Douville, A. Ribes, and D. Saint-Martin, 2011: European cold winter 2009-2010: How unusual in the instrumental record and how reproducible in the ARPEGE-Climat model? Geophys. Res. Lett., 38, 6 .

Palmer, T. N., 1999: A nonlinear dynamical perspective on climate prediction. J. Clim., $12,575-591$.

Parker, D., C. Folland, A. Scaife, J. Knight, A. Colman, P. Baines, and B. Dong, 2007: Decadal to multidecadal variability and the climate change background. $J$. Geophys. Res., 112, D18115.

Patricola, C., and K. Cook, 2010: Northern African climate at the end of the twentyfirst century: An integrated application of regional and global climate models. Clim. Dyn., 35, 193-212.

Pattanaik, D. R., and M. Rajeevan, 2010: Variability of extreme rainfall events over India during southwest monsoon season. Meteorol. Appl., 17, 88-104.

Pavelsky, T., S. Kapnick, and A. Hall, 2011: Accumulation and melt dynamics of snowpack from a multiresolution regional climate model in the central Sierra Nevada, California. J. Geophys. Res. Atmos., 116, D16115.

Pavelsky, T. M., and L. C. Smith, 2006: Intercomparison of four global precipitation data sets and their correlation with increased Eurasian river discharge to the Arctic Ocean. J. Geophys. Res. Atmos., 111, D21112.

Peduzzi, P., et al., 2012: Global trends in tropical cyclone risk. Nature Clim. Change, 2, 289-294.

Perkins, S., 2011: Biases and model agreement in projections of climate extremes over the tropical Pacific. Earth Interactions, 15, 1-36.

Perkins, S., D. Irving, J. Brown, S. Power, A. Moise, R. Colman, and I. Smith, 2012: CMIP3 ensemble climate projections over the western tropical Pacific based on model skill. Clim. Res., 51, 35-58.

Perlwitz, J., S. Pawson, R. L. Fogt, J. E. Nielsen, and W. D. Neff, 2008: Impact of stratospheric ozone hole recovery on Antarctic climate. Geophys. Res. Lett., 35, doi: 10.1029/2008gl033317.

Petersen, K. L., 1994: A warm and wet Little Climatic Optimum and a cold and dry Little Ice Age in the southern Rocky Mountains, U.S.A. Clim. Change, 26, 243269.

Petoukhov, V., and V. A. Semenov, 2010: A link between reduced Barents-Kara sea ice and cold winter extremes over northern continents. J. Geophys. Res. Atmos. 115, D21111.

Pezza, A. B., T. Durrant, I. Simmonds, and I. Smith, 2008: Southern Hemisphere synoptic behavior in extreme phases of SAM, ENSO, sea ice extent, and southern Australia rainfall. J. Clim., 21, 5566-5584.

Pezzulli, S., D. Stephenson, and A. Hannachi, 2005: The variability of seasonality. J. Clim., 18, 71-88.

Pfahl, S., and H. Wernli, 2012: Quantifying the relevance of atmospheric blocking for co-located temperature extremes in the Northern Hemisphere on (sub-)daily time scales. Geophys. Res. Lett., doi:10.1029/2012GL052261.

Philip, S., and G. Van Oldenborgh, 2006: Shifts in ENSO coupling processes under global warming. Geophys. Res. Lett., 33, doi: 10.1029/2006GL026196.

Picard, G., F. Domine, G. Krinner, L. Arnaud, and E. Lefebvre, 2012: Inhibition of the positive snow-albedo feedback by precipitation in interior Antarctica Nature Clim. Change, doi:10.1038/NCLIMATE1590.

Pinto, J. G., M. K. Karreman, K. Born, P. M. Della-Marta, and M. Klawa, 2012: Loss potentials associated with European windstorms under future climate conditions. Clim. Res., 54, 1-20.

Pinto, J. G., U. Ulbrich, G. C. Leckebusch, T. Spangehl, M. Reyers, and S. Zacharias, 2007: Changes in storm track and cyclone activity in three SRES ensemble experiments with the ECHAM5/MPI-OM1 GCM. Clim. Dyn., 29, 195-210.

Pitman, A. J., and S. E. Perkins, 2008: Regional projections of future seasonal and annual changes in rainfall and temperature over Australia based on skillselected AR4 models. Earth Interact., 12, 1-50.

Plumb, R. A., 1977: The interaction of two internal waves with the mean flow: Implications for the theory of the quasi-biennial oscillation. J. Atmos. Sci, 34, 1847-1858.

Pohl, B., N. Fauchereau, C. Reason, and M. Rouault, 2010: Relationships between the Antarctic Oscillation, the Madden - Julian Oscillation, and ENSO, and Consequences for Rainfall Analysis. J. Clim., 23, 238-254.

Polcher, J., et al., 2011: AMMA's contribution to the evolution of prediction and decision-making systems for West Africa. Atmos. Sci. Lett., 12, 2-6.

Polvani, L. M., M. Previdi, and C. Deser, 2011: Large cancellation, due to ozone recovery, of future Southern Hemisphere atmospheric circulation trends. Geophys. Res. Lett., 38, doi: 10.1029/2011gl046712.
Polyakov, I., V. Alexeev, U. Bhatt, E. Polyakova, and X. Zhang, 2010: North Atlantic warming: Patterns of long-term trend and multidecadal variability. Clim. Dyn., 34, 439-457.

Polyakov, I. V., et al., 2003: Variability and trends of air temperature and pressure in the maritime Arctic, 1875-2000. J. Clim., 16, 2067-2077.

Poore, R. Z., M. J. Pavich, and H. D. Grissino-Mayer, 2005: Record of the North American southwest monsoon from Gulf of Mexico sediment cores. Geology, 33, 209-212.

Popova, V. V., and A. B. Shmakin, 2010: Regional structure of surface-air temperature fluctuatoons in Northern Eurasia in the latter half of the 20th and early 21st centuries. Izvestiya Atmos. Ocean. Phys., 46, 144-158.

Power, S., and R. Colman, 2006: Multi-year predictability in a coupled general circulation model. Clim. Dyn., 26, 247-272.

Power, S., M. Haylock, R. Colman, and X. Wang, 2006: The predictability of interdecadal changes in ENSO activity and ENSO teleconnections. J. Clim., 19, 4755-4771.

Power, S., T. Casey, C. Folland, A. Colman, and V. Mehta, 1999: Inter-decadal modulation of the impact of ENSO on Australia. Clim. Dyn., 15, 319-324.

Power, S. B., and I. N. Smith, 2007: Weakening of the Walker Circulation and apparent dominance of El Nino both reach record levels, but has ENSO really changed? Geophys. Res. Lett., 34, L18702.

Prat, O. P., and B. R. Nelson, 2012: Precipitation contribution of tropical cyclones in the Southeastern United States from 1998 to 2009 using TRMM satellite data. J. Clim., 26, 1047-1062.

Qian, J.-H., 2008: Why precipitation is mostly concentrated over islands in the Maritime Continent. J. Atmos. Sci., 65, 1428-1441.

Qian, J.-H., A. W. Robertson, and V. Moron, 2010a: Interactions among ENSO, the Monsoon, and Diurnal Cycle in rainfall variability over Java, Indonesia. J. Atmos. Sci., 67, 3509-3524.

Qian, Y., S. J. Ghan, and L. R. Leung, 2010b: Downscaling hydroclimate changes over the Western US based on CAM subgrid scheme and WRF regional climate simulations. Int. J. Climatol., 30, 675-693.

Quadrelli, R., and J. M. Wallace, 2004: A simplified linear framework for interpreting patterns of Northern Hemisphere wintertime climate variability. J. Clim., 17, 3728-3744.

Quintana, J. M., and P. Aceituno, 2012: Changes in the rainfall regime along the extratropical west coast of South America (Chile): 30-43ㅇ. Atmosfera, 25, 1-22.

Rabatel, A., et al., 2013: Current state of glaciers in the tropical Andes: A multicentury perspective on glacier evolution and climate change. Cryosphere, 7, 81-102.

Raia, A., and I. F. A. Cavalcanti, 2008: The life cycle of the South American Monsoon System. J. Clim., 21, 6227-6246.

Raible, C., 2007: On the relation between extremes of midlatitude cyclones and the atmospheric circulation using ERA40. Geophys. Res. Lett., 34, doi: 10.1029/2006GL029084.

Raible, C. C., B. Ziv, H. Saaroni, and M. Wild, 2010: Winter synoptic-scale variability over the Mediterranean Basin under future climate conditions as simulated by the ECHAM5. Clim. Dyn., 35, 473-488.

Raible, C. C., P. M. Della-Marta, C. Schwierz, H. Wernli, and R. Blender, 2008: Northern Hemisphere extratropical cyclones: A comparison of detection and tracking methods and different reanalyses. Mon. Weather Rev., 136, 880-897.

Rajeevan, M., J. Bhate, and A. K. Jaswal, 2008: Analysis of variability and trends of extreme rainfall events over India using 104 years of gridded daily rainfall data. Geophys. Res. Lett., 35, doi: 10.1029/2008gl035143.

Rajendran, K., and A. Kitoh, 2008: Indian summer monsoon in future climate projection by a super high-resolution global model. Curr. Sci., 95, 1560-1569.

Ramanathan, V., et al., 2005: Atmospheric brown clouds: Impacts on South Asian climate and hydrological cycle. Proc. Natl. Acad. Sci. U.S.A., doi: 10.1073/ pnas.0500656102, 5326-5333.

Raphael, M. N., and M. M. Holland, 2006: Twentieth century simulation of the southern hemisphere climate in coupled models. Part 1: Large scale circulation variability. Clim. Dyn., 26, 217-228.

Rasmussen, R., et al., 2011: High-resolution coupled climate runoff simulations of seasonal snowfall over Colorado; A process study of current and warmer climate. J. Clim., 24, 3015-3048.

Rauscher, S. A., F. Giorgi, N. S. Diffenbaugh, and A. Seth, 2008: Extension and Intensification of the Meso-American mid-summer drought in the twenty-first century. Clim. Dyn., 31, 551-571. 
Rawlins, M. A., et al., 2010: Analysis of the Arctic system for freshwater cycle intensification: Observations and expectations. J. Clim., 23, 5715-5737.

Re, M., and V. Barros, 2009: Extreme rainfalls in SE South America. Clim. Change, 96, 119-136.

Reboita, M. S., T. Ambrizzi, and R. P. da Rocha, 2009: Relationship between the southern annular mode and southern hemisphere atmospheric systems. Rev. Brasil. Meteorol., 24, doi: 10.1590/S0102-77862009000100005.

Reisinger, A., A. B. Mullan, M. Manning, D. Wratt, and R. Nottage, 2010: Global and local climate change scenarios to support adaptation in New Zealand. In: Climate Change Adaptation in New Zealand: Future Scenarios and Some Sectoral Perspectives [R. A. C. Nottage, D. S. Wratt, J. F. Bornman, and K. Jones (eds.)] VUW Press, Wellington, New Zealand, pp. 26-43.

Rind, D., 2008: The consequences of not knowing low-and high-latitude climate sensitivity. Bull. Am. Meteorol. Soc., 89, 855-864.

Rinke, A., et al., 2006: Evaluation of an ensemble of Arctic regional climate models: Spatiotemporal fields during the SHEBA year. Clim. Dyn., 26, 459-472.

Risbey, J. S., M. J. Pook, P. C. McIntosh, M. C. Wheeler, and H. H. Hendon, 2009: On the remote drivers of rainfall variability in Australia. Mon. Weather Rev., 137, 3233-3253.

Riviere, G., 2011: A dynamical interpretation of the poleward shift of the jet streams in global warming scenarios. J. Atmos. Sci, 68, 1253-1272.

Robertson, A. W., et al., 2011: The Maritime Continent monsoon. In: The Global Monsoon System: Research and Forecast, 2nd ed. [C. P. Chang, Y. Ding, N. C. Lau, R. H. Johnson, B. Wang and T. Yasunari (eds.)] World Scientific Singapore, pp. 85-98.

Robinson, W. A., 2006: On the self-maintenance of midlatitude jets. J. Atmos. Sci, 63, 2109-2122.

Rodrigues, R. R., R. J. Haarsma, E. J. D. Campos, and T. Ambrizzi, 2011: The impacts of inter-El Niño variability on the tropical Atlantic and northeast Brazil climate. J. Clim., 24, 3402-3422.

Rodriguez-Fonseca, B., et al., 2011: Interannual and decadal SST-forced responses of the West African monsoon. Atmos. Sci. Lett., 12, 67-74.

Rosenfeld, D., M. Clavner, and R. Nirel, 2011: Pollution and dust aerosols modulating tropical cyclones intensities. Atmos. Res., 102, 66-76.

Rotstayn, L., and U. Lohmann, 2002: Tropical rainfall trends and the indirect aerosol effect. J. Clim., 15, 2103-2116.

Rotstayn, L. D., et al., 2007: Have Australian rainfall and cloudiness increased due to the remote effects of Asian anthropogenic aerosols? J. Geophys. Res. Atmos., 112, D09202.

Rotstayn, L. D., et al., 2009: Improved simulation of Australian climate and ENSOrelated climate variability in a GCM with an interactive aerosol treatment. Int. J. Climatol., doi:10.1002/joc.1952.

Rouault, M., P. Florenchie, N. Fauchereau, and C. Reason, 2003: South East tropical Atlantic warm events and southern African rainfall. Geophys. Res. Lett., 30, doi: 10.1029/2002GL014840.

Rowell, D. P., 2011: Sources of uncertainty in future changes in local precipitation. Clim. Dyn., 39, 1929-1950.

Rowell, D. P., 2013: Simulating SST teleconnections to Africa: What is the state of the art? J. Clim., doi:10.1175/jcli-d-12-00761.1.

Roxy, M., N. Patil, K. Ashok, and K. Aparna, 2013: Revisiting the Indian summer monsoon-ENSO links in the IPCC AR4 projections: A cautionary outlook. Global Planet. Change, doi:10.1016/j.gloplacha.2013.02.003, early on-line release.

Rupa Kumar, K., et al., 2006: High-resolution climate change scenarios for India for the 21st century. Curr. Sci., 90, 334-345.

Rusticucci, M., and M. Renom, 2008: Variability and trends in indices of qualitycontrolled daily temperature extremes in Uruguay. Int. J. Climatol., 28, 10831095.

Rusticucci, M., J. Marengo, O. Penalba, and M. Renom, 2010: An intercomparison of model-simulated in extreme rainfall and temperature events during the last half of the twentieth century. Part 1: Mean values and variability. Clim. Change, 98, 493-508.

Ruti, P., and A. Dell'Aquila, 2010: The twentieth century African easterly waves in reanalysis systems and IPCC simulations, from intra-seasonal to inter-annual variability. Clim. Dyn., 35, 1099-1117.

Sabade, S., A. Kulkarni, and R. Kripalani, 2011: Projected changes in South Asian summer monsoon by multi-model global warming experiments. Theor. Appl. Climatol., 103, 543-565.
Saenger, C., A. Cohen, D. Oppo, R. Halley, and J. Carilli, 2009: Surface-temperature trends and variability in the low-latitude North Atlantic since 1552. Nature Geosci., 2, 492-495.

Saji, N. H., B. N. Goswami, P. N. Vinayachandran, and T. Yamagata, 1999: A dipole mode in the tropical Indian Ocean. Nature, 401, 360-363.

Salahuddin, A., and S. Curtis, 2011: Climate extremes in Malaysia and the equatorial South China Sea. Global Planet. Change, 78, 83-91.

Salathe Jr, E. P., L. R. Leung, Y. Qian, and Y. Zhang, 2010: Regional climate model projections for the State of Washington. Clim. Change, 102, 51-75.

Salinger, M. J., J. A. Renwick, and A. B. Mullan, 2001: Interdecadal Pacific Oscillation and South Pacific climate. Int. J. Climatol., 21, 1705-1722.

Sampe, T., and S.-P. Xie, 2010: Large-scale dynamics of the Meiyu-Baiu rainband: Environmental forcing by the westerly jet. J. Climate, 23, 113-134.

Sansom, P. G., D. B. Stephenson, C. A. T. Ferro, G. Zappa, and L. Shaffrey, 2013: Simple uncertainty frameworks for selecting weighting schemes and interpreting multimodel ensemble climate change experiments. J. Clim., doi:10.1175/JCLI-D-1200462.1.

Santer, B. D., et al., 2007: Identification of human-induced changes in atmospheric moisture content. Proc. Natl. Acad. Sci. U.S.A., 104, 15248-15253.

Sato, T., F. Kimura, and A. Kitoh, 2007: Projection of global warming onto regional precipitation over Mongolia using a regional climate model. J. Hydrol., 333, 144-154.

Scaife, A., et al., 2011a: Climate change projections and stratosphere-troposphere interaction. Clim. Dyn., 38, 2089-2097.

Scaife, A., et al., 2009: The CLIVAR C20C project: Selected twentieth century climate events. Clim. Dyn., 33, 603-614.

Scaife, A. A., J. R. Knight, G. K. Vallis, and C. K. Folland, 2005: A stratospheric influence on the winter NAO and North Atlantic surface climate. Geophys. Res. Lett., 32, doi: 10.1029/2005gl023226.

Scaife, A. A., C. K. Folland, L. V. Alexander, A. Moberg, and J. R. Knight, 2008: European climate extremes and the North Atlantic Oscillation. J. Clim., 21, 72-83.

Scaife, A. A., T. Wollings, J. Knight, G. Martin, and T. Hinton, 2010: Atmospheric blocking and mean biases in 18 climate models. Journal of Climate, 23, 61436152.

Scaife, A. A., et al., 2011b: Improved Atlantic winter blocking in a climate model. Geophys. Res. Lett., 38, L23703.

Scarchilli, C., M. Frezzotti, and P. Ruti, 2011: Snow precipitation at four ice core sites in East Antarctica: Provenance, seasonality and blocking factors. Clim. Dyn., 37, 2107-2125.

Schimanke, S., J. Koerper, T. Spangehl, and U. Cubasch, 2011: Multi-decadal variability of sudden stratospheric warmings in an AOGCM. Geophys. Res. Lett., 38, L01801.

Schneider, D., C. Deser, and Y. Okumura, 2012: An assessment and interpretation of the observed warming of West Antarctica in the austral spring. Clim. Dyn., 38, 323-347.

Schneider, N., and B. Cornuelle, 2005: The forcing of the Pacific decadal oscillation. J. Clim., 18, 4355-4373.

Schneider, T., P. A. O'Gorman, and X. J. Levine, 2010: Water vapor and the dynamics of climate changes. Rev. Geophys., 48, RG3001.

Schott, F. A., S.-P. Xie, and J. P. McCreary, 2009: Indian Ocean circulation and climate variability. Rev. Geophys., 47, RG1002.

Schubert, J. J., B. Stevens, and T. Crueger, 2013: The Madden-Julian Oscillation as simulated by the MPI Earth System Model: Over the last and into the next millennium. J. Adv. Model. Earth Syst., 5, 71-84.

Schulz, N., J. P. Boisier, and P. Aceituno, 2012: Climate change along the arid coast of northern Chile. Int. J. Climatol., 32, 1803-1814.

Screen, J. A., I. Simmonds, C. Deser, and R. Tomas, 2012: The atmospheric response to three decades of observed Arctic sea ice loss. J. Clim., 26, 1230-1248.

Seager, R., and G. Vecchi, 2010: Greenhouse warming and the 21st century hydroclimate of southwestern North America. Proc. Natl. Acad. Sci. U.S.A., 107, 21277-21282.

Seager, R., Y. Kushnir, M. Ting, M. Cane, N. Naik, and J. Miller, 2008: Would advance knowledge of 1930s SSTs have allowed prediction of the dust bowl drought? J. Clim., 21, 3261-3281.

Seager, R., N. Naik, and L. Vogel, 2012: Does Global Warming Cause Intensified Interannual Hydroclimate Variability? J. Clim., 25, 3355-3372

Seager, R., et al., 2007: Model projections of an imminent transition to a more arid climate in southwestern North America. Science, 316, 1181-1184.

Seager, R., et al., 2009: Mexican drought: An observational modeling and tree ring study of variability and climate change. Atmosfera, 22, 1-31. 
Seidel, D. J., Q. Fu, W. J. Randel, and T. J. Reichler, 2008: Widening of the tropical belt in a changing climate. Nature Geosci, 1, 21-24.

Seierstad, I. A., and J. Bader, 2009: Impact of a projected future Arctic Sea Ice reduction on extratropical storminess and the NAO. Clim. Dyn., 33, 937-943.

Semenov, V. A., 2007: Structure of temperature variability in the high latitudes of the Northern Hemisphere. Izvestiya Atmos. Ocean. Phys., 43, 687-695.

Sen Gupta, A., A. Ganachaud, S. McGregor, J. N. Brown, and L. Muir, 2012: Drivers of the projected changes to the Pacific Ocean equatorial circulation. Geophys. Res. Lett., 39, L09605.

Sen Roy, S., 2009: A spatial analysis of extreme hourly precipitation patterns in India. Int. J. Climatol., 29, 345-355.

Seneviratne, S., et al., 2010: Investigating soil moisture-climate interactions in a changing climate: A review. Earth Sci. Rev., 95, 125-161.

Seneviratne, S. I., et al., 2012: Changes in climate extremes and their impacts on the natural physical environment. In: Managing the Risks of Extreme Events and Disasters to Advance Climate Change Adaptation. A Special Report of Working Groups I and II of the Intergovernmental Panel on Climate Change (IPCC) [C. B. Field, V. Barros, T. F. Stocker, D. Qin, D. J. Dokken, K. L. Ebi, M. D. Mastrandrea, K. J. Mach, G. -K. Plattner, S. K. Allen, M. Tignor and P. M. Midgley (eds.)]. Cambridge University Press, Cambridge, United Kingdom, and New York, NY, USA, pp. $109-230$.

Servain, J., I. Wainer, J. McCreary, and A. Dessier, 1999: Relationship between the equatorial and meridional modes of climatic variability in the tropical Atlantic. Geophys. Res. Lett., 26, 485-488.

Seth, A., M. Rojas, and S. A. Rauscher, 2010: CMIP3 projected changes in the annual cycle of the South American Monsoon. Clim. Change, 98, 331-357.

Seth, A., S. A. Rauscher, M. Rojas, A. Giannini, and S. J. Camargo, 2011: Enhanced spring convective barrier for monsoons in a warmer world? Clim. Change, 104, 403-414.

Sheffield, J., and E. F. Wood, 2008: Projected changes in drought occurrence under future global warming from multi-model, multi-scenario, IPCC AR4 simulations. Clim. Dyn., 31, 79-105.

Shi, G., J. Ribbe, W. Cai, and T. Cowan, 2008a: An interpretation of Australian rainfall projections. Geophys. Res. Lett., 35, L02702.

Shi, G., W. Cai, T. Cowan, J. Ribbe, L. Rotstayn, and M. Dix, 2008b:Variability and trend of North West Australia rainfall: Observations and coupled climate modeling. J. Clim., 21, 2938-2959.

Shongwe, M., G. van Oldenborgh, B. van den Hurk, and M. van Aalst, 2011: Projected changes in mean and extreme precipitation in Africa under global warming. Part II: East Africa. J. Clim., 24, 3718-3733.

Shongwe, M. E., G. J. van Oldenborgh, B. van den Hurk, B. de Boer, C. A. S. Coelho, and M. K. van Aalst, 2009: Projected changes in mean and extreme precipitation in Africa under global warming. Part I: Southern Africa. J. Clim., 22, 3819-3837.

Sigmond, M., and J. F. Scinocca, 2010: The influence of the basic state on the Northern Hemisphere circulation response to climate change. J. Clim., 23, 1434-1446.

Sillmann, J., M. Croci-Maspoli, M. Kallache, and R. W. Katz, 2011: Extreme cold winter temperatures in Europe under the influence of North Atlantic atmospheric blocking. J. Clim., 24, 5899-5913.

Sillmann, J., V. V. Kharin, X. Zhang, F. W. Zwiers, and D. Bronaugh, 2013: Climate extremes indices in the CMIP5 multimodel ensemble: Part 1. Model evaluation in the present climate. J. Geophys. Res. Atmos., 118, 1716-1733.

Silva, A. E., and L. M. V. Carvalho, 2007: Large-scale index for South America Monsoon (LISAM). Atmos. Sci. Lett., 8, 51-57.

Silva, V. B. S., and V. E. Kousky, 2012: The South American Monsoon System: Climatology and variability. Chapter 5 in: Modern Climatology [S.-Y.Wang (ed.)], pp 123-152.

Sinha, A., et al., 2011: A global context for megadroughts in monsoon Asia during the past millennium. Quat. Sci. Rev., 30, 47-62.

Skansi, M. d. I. M., et al., 2013: Warming and wetting signals emerging from analysis of changes in climate extreme indices over South America. Global Planet. Change, 100, 295-307.

Smirnov, D., and D. Vimont, 2011: Variability of the Atlantic Meridional Mode during the Atlantic hurricane season. J. Clim., 24, 1409-1424.

Smith, D. M., R. Eade, N. J. Dunstone, D. Fereday, J. M. Murphy, H. Pohlmann, and A. A. Scaife, 2010: Skilful multi-year predictions of Atlantic hurricane frequency. Nature Geosci, 3, 846-849.

Smith, I., and E. Chandler, 2010: Refining rainfall projections for the Murray Darling Basin of south-east Australia - the effect of sampling model results based on performance. Clim. Change, 102, 377-393.
Smith, I. N., and B. Timbal, 2012: Links between tropical indices and southern Australian rainfall. Int. J. Climatol., 32, 33-40.

Smith, I. N., L. Wilson, and R. Suppiah, 2008: Characteristics of the northern Australian rainy season. J. Clim., 21, 4298-4311.

Smith, I. N., A. F. Moise, and R. Colman, 2012a: Large scale circulation features in the tropical Western Pacific and their representation in climate models. J. Geophys. Res., 117, doi: 10.1029/2011JD016667.

Smith, K. L., L. M. Polvani, and D. R. Marsh, 2012b: Mitigation of 21st century Antarctic sea ice loss by stratospheric ozone recovery. Geophys. Res. Lett., 39, doi: 10.1029/2012GL053325.

Soares, W. R., and J. A. Marengo, 2009: Assessments of moisture fluxes east of the Andes in South America in a global warming scenario. Int. J. Climatol., 29, 1395-1414.

Sobel, A., and S. Camargo, 2011: Projected future seasonal changes in tropical summer climate. J. Clim., 24, 473-487.

Sohn, B., and S. Park, 2010: Strengthened tropical circulations in past three decades inferred from water vapor transport. J. Geophys. Res. Atmos., 115, doi: 10.1029/2009JD013713.

Solman, S., M. Nuñez, and M. Cabré, 2008: Regional climate change experiments over southern South America. I: Present climate. Clim. Dyn., 30, 533-552.

Solman, S., et al., 2013: Evaluation of an ensemble of regional climate model simulations over South America driven by the ERA-Interim reanalysis: Model performance and uncertainties. Clim. Dyn., doi:10.1007/s00382-013-1667-2, 1-19.

Solman, S. A., and H. Le Treut, 2006: Climate change in terms of modes of atmospheric variability and circulation regimes over southern South America. Clim. Dyn., 26, 835-854.

Solomon, A., and M. Newman, 2011: Decadal predictability of tropical Indo-Pacific Ocean temperature trends due to anthropogenic forcing in a coupled climate model. Geophys. Res. Lett., 38, doi: 10.1029/2010GL045978.

Son, S. W., and S. Y. Lee, 2005: The response of westerly jets to thermal driving in a primitive equation model. J. Atmos. Sci., 62, 3741-3757.

Son, S. W., et al., 2010: Impact of stratospheric ozone on Southern Hemisphere circulation change: A multimodel assessment. J. Geophys. Res., 115, D00M07.

Sörensson, A. A., C. Menéndez, R. Ruscica, P. Alexander, P. Samuelsson, and U. Willén, 2010: Projected precipitation changes in South America: A dynamical downscaling within CLARIS. . Meteorol. Z., 19, 347-355.

Sperber, K., and H. Annamalai, 2008: Coupled model simulations of boreal summer intraseasonal (30-50 day) variability, Part 1: Systematic errors and caution on use of metrics. Clim. Dyn., 31, 345-372.

Sperber, K. R., et al., 2012: The Asian summer monsoon: An intercomparison of CMIP5 vs. CMIP3 simulations of the late 20th century. Clim. Dyn., doi:10.1007/ s00382-012-1607-6, 1-34.

Stammerjohn, S. E., D. G. Martinson, R. C. Smith, X. Yuan, and D. Rind, 2008: Trends in Antarctic annual sea ice retreat and advance and their relation to El NiñoSouthern Oscillation and Southern Annular Mode variability. J. Geophys. Res., 113, C03590.

Stephenson, D., A. Hannachi, and A. O'Neill, 2004: On the existence of multiple climate regimes. Q. J. R. Meteorol. Soc., 130, 583-605.

Stephenson, D., V. Pavan, M. Collins, M. Junge, and R. Quadrelli, 2006: North Atlantic Oscillation response to transient greenhouse gas forcing and the impact on European winter climate: A CMIP2 multi-model assessment. Clim. Dyn., 27, 401-420.

Stevenson, S., B. Fox-Kemper, M. Jochum, R. Neale, C. Deser, and G. Meehl, 2012: Will there be a significant change to El Nino in the twenty-first century? J. Clim., 25, 2129-2145.

Stevenson, S. L., 2012: Significant changes to ENSO strength and impacts in the twenty-first century: Results from CMIP5. Geophys. Res. Lett., doi:10.1029/2012GL052759.

Stoner, A. M. K., K. Hayhoe, and D. J. Wuebbles, 2009: Assessing General Circulation Model simulations of atmospheric teleconnection patterns. J. Clim., 22, 43484372.

Stowasser, M., H. Annamalai, and J. Hafner, 2009: Response of the South Asian summer monsoon to global warming: Mean and synoptic systems. J. Clim., 22, 1014-1036.

Strong, C., G. Magnusdottir, and H. Stern, 2009: Observed feedback between winter sea ice and the North Atlantic Oscillation. J. Clim., 22, 6021-6032.

Sugi, M., and J. Yoshimura, 2012: Decreasing trend of tropical cyclone frequency in 228-year high-resolution AGCM simulations. Geophys. Res. Lett., 39, L19805. 
Sugi, M., H. Murakami, and J. Yoshimura, 2009: A reduction in global tropical cyclone frequency due to global warming. Sola, 5, 164-167.

Sugi, M., H. Murakami, and J. Yoshimura, 2012: On the mechanism of tropical cyclone frequency changes due to global warming. J. Meteorol. Soc. Jpn., 90A, 397-408.

Suhaila, J., S. M. Deni, W. Z. W. Zin, and A. A. Jemain, 2010: Spatial patterns and trends of daily rainfall regime in Peninsular Malaysia during the southwest and northeast monsoons: 1975-2004. Meteorol. Atmos. Phys., 110, 1-18.

Sun, J., H. Wang, and W. Yuan, 2008: Decadal variations of the relationship between the summer North Atlantic Oscillation and middle East Asian air temperature. J. Geophys. Res. Atmos., 113, D15107.

Sun, Y., and Y. H. Ding, 2010: A projection of future changes in summer precipitation and monsoon in East Asia. Science China Earth Sciences, 53, 284-300.

Sung, M.-K., G.-H. Lim, and J.-S. Kug, 2010: Phase asymmetric downstream development of the North Atlantic Oscillation and its impact on the East Asian winter monsoon. J. Geophys. Res., 115, doi: 10.1029/2009JD013153.

Sutton, R. T., and B. Dong, 2012: Atlantic Ocean influence on a shift in European climate in the 1990s. Nature Geosci, 5, 788-792.

Swart, N. C., and J. C. Fyfe, 2012: Observed and simulated changes in the Southern Hemisphere surface westerly wind-stress. Geophys. Res. Lett., 39, L16711.

Takahashi, K., and D. S. Battisti, 2007: Processes controlling the mean tropical Pacific precipitation pattern. Part II: The SPCZ and the southeast Pacific dry zone. J. Clim., 20, 5696-5706.

Takahashi, K., A. Montecinos, K. Goubanova, and B. Dewitte, 2011: ENSO regimes: Reinterpreting the canonical and Modoki El Nino. Geophys. Res. Lett., 38, doi: 10.1029/2011 gl047364.

Takaya, K., and H. Nakamura, 2005: Mechanisms of intraseasonal amplification of the cold Siberian high. J. Atmos. Sci., 62, 4423-4440.

Tanarhte, M., P. Hadjinicolaou, and J. Lelieveld, 2012: Intercomparison of temperature and precipitation data sets based on observations in the Mediterranean and the Middle East. J. Geophys. Res. Atmos., 117, doi: 10.1029/2011JD017293.

Tangang, F. T., L. Juneng, and S. Ahmad, 2007: Trend and interannual variability of temperature in Malaysia: 1961-2002. Theor. Appl. Climatol., 89, 127-141.

Tangang, F. T., et al., 2008: On the roles of the northeast cold surge, the Borneo vortex, the Madden-Julian Oscillation, and the Indian Ocean Dipole during the extreme 2006/2007 flood in southern Peninsular Malaysia. Geophys. Res. Lett., 35, L14S07.

Taylor, C., A. Gounou, F. Guichard, P. Harris, R. Ellis, F. Couvreux, and M. De Kauwe, 2011a: Frequency of Sahelian storm initiation enhanced over mesoscale soilmoisture patterns. Nature Geosci. , 4, 430-433.

Taylor, C., et al., $2011 \mathrm{~b}$ : New perspectives on land-atmosphere feedbacks from the African Monsoon Multidisciplinary Analysis. Atmos. Sci. Lett., 12, 38-44.

Taylor, K. E., R. J. Stouffer, and G. A. Meehl, 2011c: An overview of CMIP5 and the experiment design. Bull. Am. Meteorol. Soc., 93, 485-498.

Taylor, M. A., F. S. Whyte, T. S. Stephenson, and C. J.D, 2013:Why dry? Investigating the future evolution of the Caribbean Low Level Jet to explain projected Caribbean drying. Int. J. Climatol., 33, 784-792.

Taylor, M. A., T. S. Stephenson, A. Owino, A. A. Chen, and J. D. Campbell, 2011d: Tropical gradient influences on Caribbean rainfall. J. Geophys. Res., 116, D00Q08.

Tedeschi, R. G., I. F. A. Cavalcanti, and A. M. Grimm, 2013: Influences of two types of ENSO on South American precipitation. Int. J. Climatol., 33, 1382-1400.

Thomas, E. R., G. J. Marshall, and J. R. McConnell, 2008: A doubling in snow accumulation in the western Antarctic Peninsula since 1850. Geophys. Res. Lett., 35, L01706.

Thompson, D., and S. Solomon, 2009: Understanding recent stratospheric climate change. J. Clim., 22, 1934-1943.

Thompson, D. W. J., and J. M. Wallace, 1998: The Arctic Oscillation signature in the wintertime geopotential height and temperature fields. Geophys. Res. Lett., 25, $1297-1300$.

Thompson, D. W. J., and J. M. Wallace, 2000: Annular modes in the extratropical circulation. Part I: Month-to-month variability. J. Clim., 13, 1000-1016.

Thompson, D. W. J., and S. Solomon, 2002: Interpretation of recent Southern Hemisphere climate change. Science, 296, 895-899.

Thompson, D. W. J., J. M. Wallace, J. J. Kennedy, and P. D. Jones, 2010: An abrupt drop in Northern Hemisphere sea surface temperature around 1970. Nature, 467, 444-447.

Thompson, D. W. J., S. Solomon, P. J. Kushner, M. H. England, K. M. Grise, and D. J. Karoly, 2011: Signatures of the Antarctic ozone hole in Southern Hemisphere surface climate change. Nature Geosci., 4, 741-749.
Timbal, B., and J. M. Arblaster, 2006: Land cover change as an additional forcing to explain the rainfall decline in the south west of Australia. Geophys. Res. Lett., 33, L07717.

Timbal, B., and W. Drosdowsky, 2012: The relationship between the decline of Southeastern Australian rainfall and the strengthening of the subtropical ridge. Int. J. Climatol., doi:10.1002/joc.3492.

Timbal, B., J. M. Arblaster, and S. Power, 2006: Attribution of the late-twentiethcentury rainfall decline in southwest Australia. J. Clim., 19, 2046-2062.

Timmermann, A., F. F. Jin, and J. Abshagen, 2003: A nonlinear theory for El Nino bursting. J. Atmos. Sci., 60, 152-165.

Ting, M., Y. Kushnir, R. Seager, and C. Li, 2009: Forced and internal twentieth-century SST trends in the north Atlantic. J. Clim., 22, 1469-1481.

Ting, M., Y. Kushnir, R. Seager, and C. Li, 2011: Robust features of Atlantic multidecadal variability and its climate impacts. Geophys. Res. Lett., 38, L17705.

Tjernstrom, M., et al., 2004: Modeling the Arctic boundary layer: An evalutation of six ARCMIP regional-scale models with data from the SHEBA project. Bound. Layer Meteorol., 117, 337-381.

Tokinaga, H., and S. P. Xie, 2011: Weakening of the equatorial Atlantic cold tongue over the past six decades. Nature Geosci., 4, 222-226.

Tokinaga, H., S. Xie, A. Timmermann, S. McGregor, T. Ogata, H. Kubota, and Y. Okumura, 2012: Regional patterns of tropical Indo-Pacific climate change: Evidence of the Walker Circulation weakening. J. Clim., 25, 1689-1710.

Trenberth, K.E., 2011: Changes in precipitation with climate change. Climate Res., $47,123-138$.

Trenberth, K., and J. Fasullo, 2010: Simulation of present-day and twenty-firstcentury energy budgets of the southern oceans. J. Clim., 23, 440-454.

Trenberth, K., J. Fasullo, and L. Smith, 2005: Trends and variability in columnintegrated atmospheric water vapor. Clim. Dyn., 24, 741-758.

Trenberth, K., C. Davis, and J. Fasullo, 2007a: Water and energy budgets of hurricanes: Case studies of Ivan and Katrina. J. Geophys. Res. Atmos., 112, doi: 10.1029/2006JD008303.

Trenberth, K. E., D. P. Stepaniak, and J. M. Caron, 2000: The global monsoon as seen through the divergent atmospheric circulation. J. Clim., 13, 3969-3993.

Trenberth, K. E., et al., 2007b: Observations: Surface and atmospheric climate change. In: Climate Change 2007: The Physical Science Basis. Contribution of Working Group I to the Fourth Assessment Report of the Intergovernmental Panel on Climate Change [Solomon, S., D. Qin, M. Manning, Z. Chen, M. Marquis, K. B. Averyt, M. Tignor and H. L. Miller (eds.)] Cambridge University Press, Cambridge, United Kingdom and New York, NY, USA, pp. 235-336.

Trigo, R. M., I. F. Trigo, C. C. DaCamara, and T. J. Osborn, 2004: Climate impact of the European winter blocking episodes from the NCEP/NCAR Reanalyses. Clim. Dyn., 23, 17-28.

Turner, A., K. Sperber, J. Slingo, G. A. Meehl, C. R. Mechoso, M. Kimoto, and A. Giannini, 2011: Modelling monsoons: Understanding and predicting current and future behaviour. World Scientific Series on Asia-Pacific Weather and Climate, Vol. 5. The Global Monsoon System: Research and Forecast, 2nd ed. [C. P. Chang, Y. Ding, N.-C. Lau, R. H. Johnson, B. Wang and T. Yasunari (eds.)]. World Scientific Publication Company, Singapore, 608 pp.

Turner, A. G., and H. Annamalai, 2012: Climate change and the South Asian summer monsoon. Nature Clim. Change, 2, 587-595.

Turner, A. G., P. M. Inness, and J. M. Slingo, 2007a: The effect of doubled $\mathrm{CO}_{2}$ and model basic state biases on the monsoon-ENSO system. I: Mean response and interannual variability. Q. J. R. Meteorol. Soc., 133, 1143-1157.

Turner, J., 2004: The El Niño-southern oscillation and Antarctica. Int. J. Climatol., 24, 1-31.

Turner, J., J. E. Overland, and J. E. Walsh, 2007b: An Arctic and Antarctic perspective on recent climate change. Int. J. Climatol., 27, 277-293.

Turner, J., et al., 2005: Antarctic climate change during the last 50 years. Int. J. Climatol., 25, 279-294.

Tyrlis, E., and B. J. Hoskins, 2008: Aspects of a Northern Hemisphere atmospheric blocking climatology. J. Atmos. Sci., 65, 1638-1652.

Ueda, H., A. Iwai, K. Kuwako, and M. E. Hori, 2006: Impact of anthropogenic forcing on the Asian summer monsoon as simulated by eight GCMs. Geophys. Res. Lett., 33, doi: 10.1029/2005gl025336.

Ulbrich, U., and M. Christoph, 1999: A shift of the NAO and increasing storm track activity over Europe due to anthropogenic greenhouse gas forcing. Clim. Dyn., 15, 551-559.

Ulbrich, U., G. C. Leckebusch, and J. G. Pinto, 2009: Extra-tropical cyclones in the present and future climate: A review. Theor. Appl. Climatol., 96, 117-131. 
Ulbrich, U., J. G. Pinto, H. Kupfer, G. C. Leckebusch, T. Spangehl, and M. Reyers, 2008: Changing northern hemisphere storm tracks in an ensemble of IPCC climate change simulations. J. Clim., 21, 1669-1679.

Ulbrich, U., et al., 2013: Are Greenhouse Gas Signals of Northern Hemisphere winter extra-tropical cyclone activity dependent on the identification and tracking methodology? Meteorol. Z., 22, 61-68.

Ummenhofer, C. C., and M. H. England, 2007: Interannual extremes in New Zealand precipitation linked to modes of Southern Hemisphere climate variability. J. Clim., 20, 5418-5440.

Ummenhofer, C. C., A. Sen Gupta, and M. H. England, 2009a: Causes of late twentieth-century trends in New Zealand precipitation. J. Clim., 22, 3-19.

Ummenhofer, C. C., M. H. England, P. C. McIntosh, G. A. Meyers, M. J. Pook, J. S. Risbey, A. S. Gupta, and A. S. Taschetto, 2009b: What causes southeast Australia's worst droughts? Geophys. Res. Lett., 36, doi: 10.1029/2008gl036801.

van den Broeke, M. R., and N. P. M. van Lipzig, 2004: Changes in Antarctic temperature, wind and precipitation in response to the Antarctic Oscillation. Ann. Glaciol., 39, 119-126.

van Ommen, T. D., and V. Morgan, 2010: Snowfall increase in coastal East Antarctica linked with southwest Western Australian drought. Nature Geosci, 3, 267-272.

Vance, T. R., T. D. van Ommen, M. A. J. Curran, C. T. Plummer, and A. D. Moy, 2012: A millennial proxy record of ENSO and eastern Australian rainfall from the Law Dome ice core, East Antarctica. J. Clim., 26, 710-725.

Vancoppenolle, M., T. Fichefet, H. Goosse, S. Bouillon, G. Madec, and M. A. M. Maqueda, 2009: Simulating the mass balance and salinity of arctic and antarctic sea ice. 1. Model description and validation. Ocean Model., 27, 33-53.

Vasconcellos, F. C., and I. F. A. Cavalcanti, 2010: Extreme precipitation over Southeastern Brazil in the austral summer and relations with the Southern Hemisphere annular mode. Atmos. Sci. Lett., 11, 21-26.

Vautard, R., et al., 2007: Summertime European heat and drought waves induced by wintertime Mediterranean rainfall deficit. Geophys. Res. Lett., 34, doi: 10.1029/2006GL028001.

Vecchi, G., and A. Wittenberg, 2010: El Nino and our future climate: Where do we stand? WIREs Clim Change, 1, 260-270.

Vecchi, G. A., and B. J. Soden, 2007a: Global warming and the weakening of the tropical circulation. J. Clim., 20, 4316-4340.

Vecchi, G. A., and B. J. Soden, 2007b: Increased tropical Atlantic wind shear in model projections of global warming. Geophys. Res. Lett., 34, L08702.

Vecchi, G. A., B. J. Soden, A. T. Wittenberg, I. M. Held, A. Leetmaa, and M. J. Harrison, 2006:Weakening of tropical Pacific atmospheric circulation due to anthropogenic forcing. Nature, 441, 73-76.

Vera, C., and G. Silvestri, 2009: Precipitation interannual variability in South America from the WCRP-CMIP3 multi-model dataset. Clim. Dyn., 32, 1003-1014.

Vera, C., et al., 2006: Toward a unified view of the American Monsoon Systems. J. Clim., 19, 4977-5000.

Vergara, W., et al., 2007: Visualizing future climate in Latin America: Results from the application of the Earth Simulator. In: Latin America and Caribbean Region Sustainable Development Working Paper No. 30. The World Bank, Washington, DC, $82 \mathrm{pp}$.

Vial, J., and T. Osborn, 2012: Assessment of atmosphere-ocean general circulation model simulations of winter northern hemisphere atmospheric blocking. Clim. Dyn., 39, 95-112.

Vigaud, N., B. Pohl, and J. Crétat, 2012: Tropical-temperate interactions over southern Africa simulated by a regional climate model. Clim. Dyn., doi:10.1007/s00382012-1314-3, 1-22.

Vigaud, N., Y. Richard, M. Rouault, and N. Fauchereau, 2009: Moisture transport between the South Atlantic Ocean and southern Africa: Relationships with summer rainfall and associated dynamics. Clim. Dyn., 32, 113-123.

Villarini, G., and G. A. Vecchi, 2012: Twenty-first-century projections of North Atlantic tropical storms from CMIP5 models. Nature Clim. Change, 2, 604-607.

Vimont, D., M. Alexander, and A. Fontaine, 2009: Midlatitude excitation of tropical variability in the Pacific: The role of thermodynamic coupling and seasonality. J. Clim., 22, 518-534.

Vimont, D. J., and J. P. Kossin, 2007: The Atlantic Meridional Mode and hurricane activity. Geophys. Res. Lett., 34, L07709.

Vincent, E., M. Lengaigne, C. Menkes, N. Jourdain, P. Marchesiello, and G. Madec, 2011: Interannual variability of the South Pacific Convergence Zone and implications for tropical cyclone genesis. Clim. Dyn., 36, 1881-1896.

Vincent, L. A., W. A. van Wijngaarden, and R. Ropkinson, 2007: Surface temperature and humidity trends in Canda for 1953-2005. J. Clim., 20, 5100-5113.
Vizy, E., and K. Cook, 2002: Development and application of a mesoscale climate model for the tropics: Influence of sea surface temperature anomalies on the West African monsoon. J. Geophys. Res. Atmos., 107, ACL 2-1-ACL 2-22.

Vuille, M., B. Francou, P. Wagnon, I. Juen, G. Kaser, B. G. Mark, and R. S. Bradley, 2008: Climate change and tropical Andean glaciers: Past, present and future. Earth Sci. Rev., 89, 79-96.

Walsh, K., K. Mclnnes, and J. McBride, 2012: Climate change impacts on tropical cyclones and extreme sea levels in the South Pacific - A regional assessment. Global Planet. Change, 80-81, 149-164.

Wang, B., 1995: Interdecadal changes in El-Nino onset in the last four decades. J. Clim., 8, 267-285.

Wang, B., and Y. Wang, 1996: Temporal structure of the Southern Oscillation as revealed by waveform and wavelet analysis. J. Clim., 9, 1586-1598.

Wang, B., and S. I. An, 2001: Why the properties of El Nino changed during the late 1970s. Geophys. Res. Lett., 28, 3709-3712.

Wang, B., and S. I. An, 2002: A mechanism for decadal changes of ENSO behavior: Roles of background wind changes. Clim. Dyn., 18, 475-486.

Wang, B., and LinHo, 2002: Rainy season of the Asian-Pacific summer monsoon. J. Clim., 15, 386-398.

Wang, B., and Q. Ding, 2006: Changes in global monsoon precipitation over the past 56 years. Geophys. Res. Lett., 33, L06711.

Wang, B., R. G. Wu, and T. Li, 2003: Atmosphere-warm ocean interaction and its impacts on Asian-Australian monsoon variation. J. Clim., 16, 1195-1211.

Wang, B., I. S. Kang, and J. Y. Lee, 2004: Ensemble simulations of Asian-Australian monsoon variability by 11 AGCMs. J. Clim., 17, 803-818.

Wang, B., Q. Ding, and J. Jhun, 2006: Trends in Seoul (1778-2004) summer precipitation. Geophys. Res. Lett., 33, L15803.

Wang, B., J. Yang, and T. J. Zhou, 2008a: Interdecadal changes in the major modes of Asian-Australian monsoon variability: Strengthening relationship with ENSO since the late 1970s. J. Clim., 21, 1771-1789.

Wang, B., H.-J. Kim, K. Kikuchi, and A. Kitoh, 2011: Diagnostic metrics for evaluation of annual and diurnal cycles. Clim. Dyn., 37, 941-955.

Wang, B., S. Xu, and L. Wu, 2012a: Intensified Arabian Sea tropical storms. Nature, 489, E1-E2.

Wang, B., J. Liu, H.-J. Kim, P. J. Webster, and S.-Y. Yim, 2012b: Recent change of the global monsoon precipitation (1979-2008). Clim. Dyn., 39, 1123-1135.

Wang, C., S. K. Lee, and D. B. Enfield, 2007: Impact of the Atlantic warm pool on the summer climate of the Western Hemisphere. J. Clim., 20, 5021-5040.

Wang, C., S. K. Lee, and D. B. Enfield, 2008b: Climate response to anomalously large and small Atlantic warm pools during the summer. J. Clim., 21, 2437-2450.

Wang, H., 2001: The weakening of the Asian monsoon circulation after the end of 1970's. Adv. Atmos. Sci, 376-386.

Wang, L., and W. Chen, 2010: How well do existing indices measure the strength of the East Asian winter monsoon? Adv. Atmos. Sci., 27, 855-870.

Wang, L., R. Huang, L. Gu, W. Chen, and L. Kang, 2009a: Interdecadal variations of the east Asian winter monsoon and their association with quasi-stationary planetary wave activity. J. Clim., 22, 4860-4872.

Wang, L., W. Chen, W. Zhou, J. C. L. Chan, D. Barriopedro, and R. Huang, 2010: Effect of the climate shift around mid 1970s on the relationship between wintertime Ural blocking circulation and East Asian climate. Int. J. Climatol., 30, 153-158.

Wang, S. Y., R. R. Gillies, E. S. Takle, and W. J. Gutowski, 2009b: Evaluation of precipitation in the Intermountain Region as simulated by the NARCCAP regional climate models. Geophys. Res. Lett., 36, L11704.

Wang, X., C. Z. Wang, W. Zhou, D. X. Wang, and J. Song, 2011: Teleconnected influence of North Atlantic sea surface temperature on the El Niño onset. Clim. Dyn., 37, 663-676.

Wanner, H., et al., 2001: North Atlantic Oscillation-Concepts and studies. Surveys in Geophysics, 22, 321-382.

Ward, P., M. Marfai, Poerbandono, and E. Aldrian, 2011: Climate adaptation in the city of Jakarta. Chapter 13 in: Climate Adaptation and Flood Risk in Coastal Cities [J. Aerts, W. Botzen, M. Bowman, P. Ward and P. Dircke (eds.)]. Routledge Earthscan, Amsterdam, Netherlands, $330 \mathrm{pp}$.

Watanabe, S., and Y. Kawatani, 2012: Sensitivity of the QBO to mean tropical upwelling under a changing climate simulated with an Earth System Model. J. Meteorol. Soc. Jpn. II, 90A, 351-360.

Watterson, I., A. C. Hirst, and L. D. Rotstayn, 2013: A skill-score based evaluation of simulated Australian climate. Australian Meteorol. Oceanogr. J., 63, 181-190. 
Watterson, I. G., 2009: Components of precipitation and temperature anomalies and change associated with modes of the Southern Hemisphere. Int. J. Climatol., 29, 809-826.

Webster, P. J., A. M. Moore, J. P. Loschnigg, and R. R. Leben, 1999: Coupled oceanatmosphere dynamics in the Indian Ocean during 1997-98. Nature, 401, 356360.

Weller, E., and W. Cai, 2013: Realism of the Indian Ocean Dipole in CMIP5 models: The implication for climate projections. J. Clim., 26, 6649-6659.

Widlansky, M., P. Webster, and C. Hoyos, 2011: On the location and orientation of the South Pacific Convergence Zone. Clim. Dyn., 36, 561-578.

Widlansky, M. J., et al., 2013: Changes in South Pacific rainfall bands in a warming climate. Nature Clim. Change, 3, 417-423.

Wiedenmann, J. M., A. R. Lupo, I. I. Mokhov, and E.A. Tikhonova, 2002: The climatology of blocking anticyclones for the Northern and Southern Hemispheres: Block intensity as a diagnostic. J. Clim., 15, 3459-3473.

Wilcox, L. J., A. J. Charlton-Perez, and L. J. Gray 2012: Trends in Austral jet position in ensembles of high- and low-top CMIP5 models. J. Geophys. Res., doi:10.1029/2012JD017597.

Williams, A., and C. Funk, 2011: A westward extension of the warm pool leads to a westward extension of the Walker circulation, drying eastern Africa. Clim. Dyn., 37, 2417-2435.

Wilson, A. B., D. H. Bromwich, and K. M. Hines, 2012: Evaluation of Polar WRF forecasts on the Arctic System Reanalysis domain:2. Atmopsheric hydrologic cycle. J. Geophys. Res., 17, D04107.

Wittenberg, A., 2004: Extended wind stress analyses for ENSO. J. Clim., 17, 25262540.

Wittenberg, A. T., 2009: Are historical records sufficient to constrain ENSO simulations? Geophys. Res. Lett., 36, L12702.

Woollings, T., 2008: Vertical structure of anthropogenic zonal-mean atmospheric circulation change. Geophys. Res. Lett., 35, L19702.

Woollings, T., 2010: Dynamical influences on European climate: An uncertain future. Philos. Trans. R. Soc. London A, 368, 3733-3756.

Woollings, T., A. Charlton-Perez, S. Ineson, A. G. Marshall, and G. Masato, 2010: Associations between stratospheric variability and tropospheric blocking. J. Geophys. Res. Atmos., 115, D06108.

Woollings, T., J. Gregory, J. Pinto, M. Reyers, and D. Brayshaw, 2012: Response of the North Atlantic storm track to climate change shaped by ocean-atmosphere coupling. Nature Geosci, 5, 313-317.

Wu, J., and X. J. Gao, 2013: A gridded daily observation dataset over China region and comparison with the other datasets. Chin. J. Geophys (in Chinese), 56, 1102-1111.

Wu, L. G., 2007: Impact of Saharan air layer on hurricane peak intensity. Geophys. Res. Lett., 34, doi: 10.1029/2007GL029564.

Wu, Q., and X. Zhang, 2010: Observed forcing-feedback processes between Northern Hemisphere atmospheric circulation and Arctic sea ice coverage. J. Geophys. Res. Atmos., 115., doi: 10.1029/2009jd013574.

Wu, Q. G., and D. J. Karoly, 2007: Implications of changes in the atmospheric circulation on the detection of regional surface air temperature trends. Geophys. Res. Lett., 34, L08703.

Wu, R., B. P. Kirtman, and V. Krishnamurthy, 2008: An asymmetric mode of tropical Indian Ocean rainfall variability in boreal spring. J. Geophys. Res. Atmos., 113, D05104.

Wu, Y., M. Ting, R. Seager, H.-P. Huang, and M. A. Cane, 2011: Changes in storm tracks and energy transports in a warmer climate simulated by the GFDL CM2.1 model. Clim. Dyn., 37, 53-72.

Xie, P., and P. A. Arkin, 1997: Global precipitation: A 17-year monthly analysis based on gauge observations, satellite estimates, and numerical model outputs. Bull. Am. Meteorol. Soc., 78, 2539-2558.

Xie, S.-P., et al., 2007: A regional ocean-atmosphere model for Eastern Pacific climate: Toward reducing tropical biases. J. Clim., 20, 1504-1522.

Xie, S. P., and S. G. H. Philander, 1994: A coupled ocean-atmosphere model of relevance to the ITCZ in the eastern Pacific. Tellus A, 46, 340-350.

Xie, S. P., and J. A. Carton, 2004: Tropical Atlantic variability: Patterns, mechanisms, and impacts. Earth Clim. Ocean-Atmos. Interact., American Geophysical Union, 121-142.

Xie, S. P., K. Hu, J. Hafner, H. Tokinaga, Y. Du, G. Huang, and T. Sampe, 2009: Indian Ocean capacitor effect on Indo-western Pacific climate during the summer following El Niño. J. Clim., 22, 730-747.
Xie, S. P., Y. Du, G. Huang, X. T. Zheng, H. Tokinaga, K. M. Hu, and Q. Y. Liu, 2010a: Decadal shift in El Niño influences on Indo-western Pacific and east Asian climate in the 1970s. J. Clim., 23, 3352-3368.

Xie, S. P. D., C. Deser, G. A. Vecchi, J. Ma, H. Teng, and A. T. Wittenberg, 2010b: Global warming pattern formation: Sea surface temperature and rainfall. J. Clim., 23, 966-986.

Xu, Y., X.-J. Gao, and F. Giorgi, 2009: Regional variability of climate change hot-spots in East Asia. Adv. Atmos. Sci., 26, 783-792.

Xue, Y., et al., 2010: Intercomparison and analyses of the climatology of the West African Monsoon in the West African Monsoon Modeling and Evaluation project (WAMME) first model intercomparison experiment. Clim. Dyn., 35, 3-27.

Yamada, Y., K. Oouchi, M. Satoh, H. Tomita, andW. Yanase, 2010: Projection of changes in tropical cyclone activity and cloud height due to greenhouse warming: Global cloud-system-resolving approach. Geophys. Res. Lett., 37, L07709.

Yamagata, T., S. K. Behera, J.-J. Luo, S. Masson, M. Jury, and S. A. Rao, 2004: Coupled ocean-atmosphere variability in the tropical Indian Ocean. Earth Clim. OceanAtmos. Interact., American Geophysical Union, 189-212.

Yamazaki, A., and H. Itoh, 2009: Selective absorption mechanism for the maintenance of blocking. Geophys. Res. Lett., 36, L05803.

Yan, H., L. G. Sun, Y. H. Wang, W. Huang, S. C. Qiu, and C. Y. Yang, 2011: A record of the Southern Oscillation Index for the past 2,000 years from precipitation proxies. Nature Geosci., 4, 611-614.

Yang, S., and J. H. Christensen, 2012: Arctic sea ice reduction and European cold winters in CMIP5 climate change experiments. Geophys. Res. Lett., 39, L20707.

Yatagai, A., K. Kamiguchi, O. Arakawa, A. Hamada, N. Yasutomi, and A. Kitoh, 2012: APHRODITE: Constructing a long-term daily gridded precipitation dataset for Asia based on a dense network of rain gauges. Bull. Am. Meteorol. Soc., 93, 1401-1415.

Ye, Z. Q., and W. W. Hsieh, 2008: Changes in ENSO and associated overturning circulations from enhanced greenhouse gases by the end of the twentieth century. J. Clim., 21, 5745-5763.

Yeh, S.-W., Y.-G. Ham, and J.-Y. Lee, 2012: Changes in the tropical Pacific SST Trend from CMIP3 to CMIP5 and its implication of ENSO. J. Clim., 25, 7764-7771.

Yeh, S.-W., B. P. Kirtman, J.-S. Kug, W. Park, and M. Latif, 2011: Natural variability of the central Pacific El Nino event on multi-centennial timescales. Geophys. Res. Lett., 38, L02704.

Yeh, S. W., and B. P. Kirtman, 2005: Pacific decadal variability and decadal ENSO amplitude modulation. Geophys. Res. Lett., 32, L05703.

Yeh, S. W., J. S. Kug, B. Dewitte, M. H. Kwon, B. P. Kirtman, and F. F. Jin, 2009: El Nino in a changing climate. Nature, 461, 511-515.

Yeung, J. K., J. A. Smith, G. Villarini, A. A.N., M. L. Baeck, and W. F. Krajewski, 2011: Analyses of the warm season rainfall climatology of the northeastern US using regional climate model simulations and radar rainfall fields. Adv. Water Resour., 34, 184-204.

Yin, J. H., 2005: A consistent poleward shift of the storm tracks in simulations of 21st century climate. Geophys. Res. Lett., 32, 4.

Yin, L., R. Fu, E. Shevliakova, and R. Dickinson, 2012: How well can CMIP5 simulate precipitation and its controlling processes over tropical South America? Clim. Dyn., doi:10.1007/s00382-012-1582-y.

Ying, M., T. R. Knutson, H. Kamahori, and T.-C. Lee, 2012: Impacts of climate change on tropical cyclones in the Western North Pacific Basin. Part II: Late twenty-first century projections. Trop. Cyclone Res. Rev., 1, 231-241.

Yokoi, S., and Y. Takayabu, 2009: Multi-model projection of global warming impact on tropical cyclone genesis frequency over the western north Pacific. J. Meteorol. Soc. Jpn., 87, 525-538.

Yosef, Y., H. Saaroni, and P. Alpert, 2009: Trends in daily rainfall intensity over Israel 1950/1-2003/4. Open Atmos. Sci. J., 3, 196-203.

Yu, B., and F. W. Zwiers, 2010: Changes in equatorial atmospheric zonal circulations in recent decades. Geophys. Res. Lett., 37, L05701.

Yu, R. C., B. Wang, and T. J. Zhou, 2004: Tropospheric cooling and summer monsoon weakening trend over East Asia. Geophys. Res. Lett., 31, L22212.

Zahn, M., and H. von Storch, 2010: Decreased frequency of North Atlantic polar lows associated with future climate warming. Nature, 467, 309-312.

Zahn, M., and R. Allan, 2011: Changes in water vapor transports of the ascending branch of the tropical circulation. J. Geophys. Res. Atmos., 116, doi: 10.1029/2011JD016206.

Zanchettin, D., A. Rubino, and J. Jungclaus, 2010: Intermittent multidecadal-tocentennial fluctuations dominate global temperature evolution over the last millennium. Geophys. Res. Lett., 37, L14702. 
Zappa, G., L. C. Shaffrey, and K. I. Hodges, 2013a: The ability of CMIP5 models to simulate North Atlantic extratropical cyclones. J. Clim., doi:10.1175/jcli-d-1200501.1 .

Zappa, G., L. C. Shaffrey, K. I. Hodges, P. G. Sansom, and D. B. Stephenson, 2013b: A multi-model assessment of future projections of North Atlantic and European extratropical cyclones in the CMIP5 climate models. J. Clim., doi:10.1175/jcli-d12-00573.1.

Zebiak, S. E., 1993: Air-sea interaction in the equatorial Atlantic region. J. Clim., 6, 1567-1586.

Zhang, C., 2005: Madden-Julian Oscillation. Rev. Geophys., 43, RG2003.

Zhang, H., P. Liang, A. Moise, and L. Hanson, 2013a: The response of summer monsoon onset/retreat in Sumatra-Java and tropical Australia region to global warming in CMIP3 models. Clim. Dyn., 40, 377-399.

Zhang, J., U. S. Bhatt, W. V. Tangborn, and C. S. Lingle, 2007: Climate downscaling for estimating glacier mass balances in northwestern North America: Validation with a USGS benchmark glacier. Geophys. Res. Lett., 34, L21505.

Zhang, L., L. Wu, and L. Yu, 2011 a: Oceanic origin of a recent La Nia-like trend in the tropical Pacific. Adv. Atmos. Sci., 28, 1109-1117.

Zhang, L. X., and T. J. Zhou, 2011: An assessment of monsoon precipitation changes during 1901-2001. Clim. Dyn., 37, 279-296.

Zhang, M. H., and H. Song, 2006: Evidence of deceleration of atmospheric vertical overturning circulation over the tropical Pacific. Geophys. Res. Lett., 33, L12701.

Zhang, Q., Y. Guan, and H. Yang, 2008: ENSO amplitude change in observation and coupled models. Adv. Atmos. Sci., 25, 361-366.

Zhang, R., and T. L. Delworth, 2006: Impact of Atlantic multidecadal oscillations on India/Sahel rainfall and Atlantic hurricanes. Geophys. Res. Lett., 33, L17712.

Zhang, R., and T. L. Delworth, 2009: A new method for attributing climate variations over the Atlantic Hurricane Basin's main development region. Geophys. Res. Lett., 36, L06701.

Zhang, R., et al., 2013b: Have aerosols caused the observed Atlantic multidecadal variability? J. Atmos. Sci., 70, 1135-1144.

Zhang, S., and B. Wang, 2008: Global summer monsoon rainy seasons. Int. J. Climatol., 28, 1563-1578.

Zhang, X., R. Brown, L. Vincent, W. Skinner, Y. Feng, and E. Mekis, 2011 b: Canadian climate trends, 1950-2007. Canadian Biodiversity: Ecosystem Status and Trends 2012, Technical Thematic Report No. 5. Canadian Councils of Resource Ministers, Ottowa, iv $+21 p$.

Zhang, X., et al., 2005: Trends in Middle East climate extreme indices from 1950 to 2003. J. Geophys. Res. Atmos., 110, doi: 10.1029/2005JD006181.

Zhang, X. B., F. W. Zwiers, and P. A. Stott, 2006: Multimodel multisignal climate change detection at regional scale. J. Clim., 19, 4294-4307.

Zhao, M., and I. Held, 2012: TC-permitting GCM simulations of hurricane frequency response to sea surface temperature anomalies projected for the late twentyfirst century. J. Clim., 25, 2995-3009.

Zhao, M., I. M. Held, S. J. Lin, and G. A. Vecchi, 2009: Simulations of global hurricane climatology, interannual variability, and response to global warming using a 50-km resolution GCM. J. Clim., 22, 6653-6678.

Zheng, X.-T., S.-P. Xie, and Q. Liu, 2011: Response of the Indian Ocean basin mode and its capacitor effect to global warming. J. Clim., 24, 6146-6164.

Zheng, X.-T., Y. Du, L. Liu, G. Huang, and Q. Liu, 2013: Indian Ocean Dipole response to global warming in the CMIP5 multi-model ensemble. J. Clim., 26, 6067-6080.

Zheng, X. T., S. P. Xie, G. A. Vecchi, Q. Y. Liu, and J. Hafner, 2010: Indian Ocean Dipole response to global warming: Analysis of ocean-atmospheric feedbacks in a coupled model. J. Clim., 23, 1240-1253.

Zhou, T., B. Wu, and B. Wang, 2009a: How well do atmospheric general circulation models capture the leading modes of the interannual variability of the AsianAustralian monsoon? J. Clim., 22, 1159-1173.

Zhou, T., R. Yu, H. Li, and B. Wang, 2008a: Ocean forcing to changes in global monsoon precipitation over the recent half-century. J. Clim., 21, 3833-3852.

Zhou, T. J., and R. C. Yu, 2005: Atmospheric water vapor transport associated with typical anomalous summer rainfall patterns in China. J. Geophys. Res. Atmos., 110, D08104.

Zhou, T. J., and L. W. Zou, 2010: Understanding the predictability of East Asian summer monsoon from the reproduction of land-sea thermal contrast change in AMIP-type simulation. J. Clim., 23, 6009-6026.

Zhou, T. J., L. X. Zhang, and H. M. Li, 2008b: Changes in global land monsoon area and total rainfall accumulation over the last half century. Geophys. Res. Lett., 35, L16707.
Zhou, T. J., D. Y. Gong, J. Li, and B. Li, 2009b: Detecting and understanding the multidecadal variability of the East Asian Summer Monsoon-Recent progress and state of affairs. Meteorol. Z., 18, 455-467.

Zhou, T. J., et al., 2009c: The CLIVAR C20C project: Which components of the AsianAustralian monsoon circulation variations are forced and reproducible? Clim. Dyn., 33, 1051-1068.

Zhou, W., J. C. L. Chan, W. Chen, J. Ling, J. G. Pinto, and Y. Shao, 2009d: Synoptic-scale controls of persistent low temperature and icy weather over southern China in January 2008. Mon. Weather Rev., 137, 3978-3991.

Zhu, C., B. Wang, W. Qian, and B. Zhang, 2012: Recent weakening of northern East Asian summer monsoon: A possible response to global warming. Geophys. Res. Lett., 39, doi: 10.1029/2012GL051155.

Zhu, Y. L., and H. J. Wang, 2010: The Arctic and Antarctic Oscillations in the IPCC AR4 Coupled Models. Acta Meteorol. Sin., 24, 176-188. 